Fundamentals and applications of fast micro-drop impact

Claas Willem Visser 


\section{FUNDAMENTALS AND APPLICATIONS OF FAST MICRO-DROP IMPACT}

Claas Willem Visser 


\section{Graduation committee members:}

Prof. Dr. Ir. Hans Hilgenkamp (chair)

Prof. Dr. Detlef Lohse (promotor)

Assoc. Prof. Dr. Chao Sun (co-promotor)

Prof. Dr. Boris Chichkov

Prof. Dr. Ir. Jaap den Toonder

Prof. Dr. Ir. Bert Huis in 't Veld

Prof. Dr. Marcel Karperien
University of Twente

University of Twente

University of Twente

Laser Zentrum Hannover

University of Eindhoven

University of Twente

University of Twente

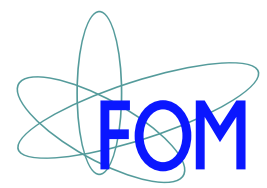

The work in this thesis was carried out at the Physics of Fluids group of the Faculty of Science and Technology of the University of Twente. It is part of the research program of the Foundation for Fundamental Research on Matter (FOM), which is financially supported by the Netherlands Organisation for Scientific Research (NWO).

Dutch title: Fundamenten en toepassingen van snelle micro-druppel inslag

Cover: Impacting micro-droplet viewed from below (see figure 4.6 for the time series). Remarkably, the fringes are caused by interference between the (curved) top surface of the droplet and the (flat) impingement surface. These fringes allow to determine the droplet's surface shape, as explained in section 4.3.

Copyricht (c) 2014. Claas Willem Visser, Enschede, The Netherlands

All rights reserved. No part of this publication may be reproduced, stored in a retrieval system, or transmitted, in any form or by any means, electronic, mechanical, photocopying, recording, or otherwise, without written permission of the author.

Printed by Gildeprint Drukkerijen, Enschede, The Netherlands ISBN: 978-90-365-3816-9 


\title{
FUNDAMENTALS AND APPLICATIONS OF FAST MICRO-DROP IMPACT
}

\author{
DISSERTATION
}

\author{
to obtain
}

the degree of doctor at the University of Twente, on the authority of the rector magnificus,

Prof. Dr. H. Brinksma,

on account of the decision of the graduation committee,

to be publicly defended

on Friday the 19th of December 2014 at 16:45 hours

by

Claas Willem Visser

Born on the 11th of September 1981

in Haarlem, The Netherlands 
This dissertation has been approved by the promotor:

Prof. Dr. rer. nat. Detlef Lohse

and the copromotor:

Assoc. Prof. Dr. Chao Sun 


\section{Contents}

1 Introduction 1

1.1 Motivation . . . . . . . . . . . . . . . . . 1

1.2 Fundamentals and recent developments . . . . . . . . . . . . 8

1.3 Research problem and scientific approach . . . . . . . . . . . . 11

1.4 Reading guide . . . . . . . . . . . . . . . . . 13

2 On highly focused supersonic microjets 17

2.1 Introduction . . . . . . . . . . . . . . . . . . 18

2.2 Experimental setup and control parameters . . . . . . . . . . 19

2.3 Jet formation and evolution . . . . . . . . . . . . . . . 20

2.4 Parameter dependence ... . . . . . . . . . . . . . 24

2.5 Summary and conclusions . . . . . . . . . . . . . 32

3 Microdroplet impact at very high velocity 37

3.1 Introduction . . . . . . . . . . . . . . . . . 38

3.2 Parameter space . . . . . . . . . . . . . . . 38

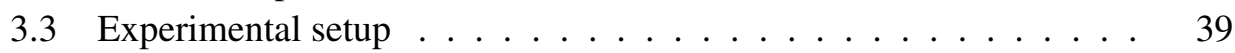

3.4 Results . . . . . . . . . . . . . . . . . 42

3.5 Conclusions ....................... 47

4 Dynamics of high-speed micro-droplet impact 51

4.1 Introduction . . . . . . . . . . . . . . . . 52

4.2 Droplet generation, visualization, and modeling . . . . . . . . . 56

4.3 Results and discussion ................. . . 60

4.4 Conclusions . . . . . . . . . . . . . . . . 76

5 Ejection regimes in picosecond LIFT of metals $\mathbf{8 5}$

5.1 Introduction . . . . . . . . . . . . . . . . . 86

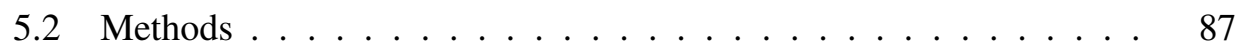

5.3 Results...................... 89 
5.4 Discussion . . . . . . . . . . . . . . . . . . 99

5.5 Conclusions ....................... 100

6 LIFT: Towards 3D printing of pure metals 105

6.1 Introduction . . . . . . . . . . . . . . . . 106

6.2 Results . . . . . . . . . . . . . . . . . . 109

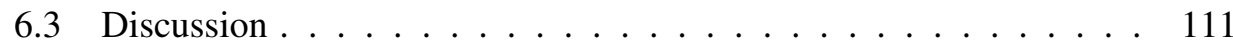

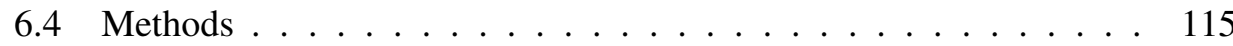

7 Optimizing cell viability in droplet-based cell deposition 121

7.1 Introduction . . . . . . . . . . . . . . . . . . 122

7.2 Results....................... 123

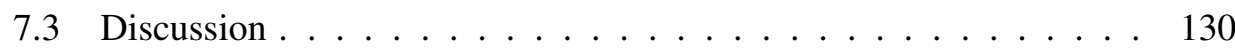

7.4 Methods . . . . . . . . . . . . . . . . . . 134

7.5 Supplementary material . . . . . . . . . . . . . 136

8 Quantifying cell adhesion by controlled microjet impingement 149

8.1 Introduction . . . . . . . . . . . . . . . . 150

8.2 Materials and methods . . . . . . . . . . . . . . . 151

8.3 Results ......................... . . . 154

8.4 Discussion . . . . . . . . . . . . . . . . 164

8.5 Conclusions . . . . . . . . . . . . . . . . . 166

8.6 Supplementary material . . . . . . . . . . . . . 168

9 Conclusions and outlook $\quad 179$

$\begin{array}{ll}\text { Summary } & 185\end{array}$

$\begin{array}{lr}\text { Samenvatting } & 189\end{array}$

$\begin{array}{ll}\text { Acknowledgements } & 193\end{array}$

$\begin{array}{ll}\text { About the author } & 197\end{array}$

$\begin{array}{ll}\text { Scientific output } & 199\end{array}$ 


\section{(1) \\ Introduction *}

\subsection{Motivation}

When staring out of the window on a rainy day, one can readily observe some of the remarkable outcomes of impact experiments with water drops. In heavy rain, the drops impact on the wet film deposited by previous drops and splash into a crown, whereas in light rain the drops form small, circular disks. Already in the early 16th century, Leonardo da Vinci noticed the remarkable splashing patterns after impact and included them in his Codex Hammer (see figure1.1 (a)). However, a systematic investigation into the impact of drops was only achieved centuries later, owing to the short time scales of the impact phenomena. For example, the impact of a 3-mm drop falling from $10 \mathrm{~cm}$ occurs in about $2 \mathrm{~ms}$, which is far too short to be observed with the naked eye. It was only after the introduction of electric flash illumination that the first systematic studies were reported by Worthington [3]. He used a bright flash in a dark room to illuminate the impacting drop and observe it by eye. Subsequently he drew the impact patterns from memory, as shown in figure 1.1 (b). Since then, dropimpact experiments on flat substrates [3,4], heated substrates [5-8], at high velocities $[9,10]$, and for small drops [11-13] have been performed; for an overview see refs. $[14,15]$. However, over the last 15 years, the understanding of drop impact has pro-

${ }^{*}$ Based on: H. Gelderblom, C.W. Visser, C. Sun, D. Lohse, 2012. The parts used for this chapter were written in draft by CWV. 
(a)

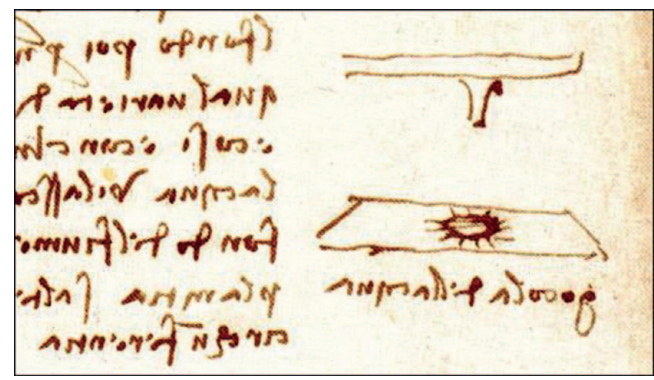

(b)

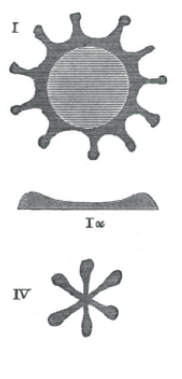

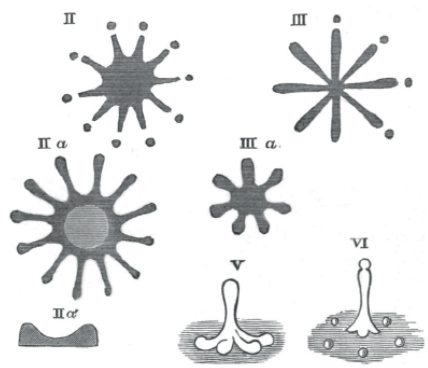

Figure 1.1: Visualizations of drop impact. (a) Crushing of a water drop falling on a flat dense substrate. Drawing in the margin of folio $33 \mathrm{r}$ in the Codex Hammer by Leonardo da Vinci (1508), who already noted the axisymmetry of an impacted drop imprint pattern and the existence of radial fingers [1] (description from [2]). (b) Drawings of the impact of a boiled milk drop on a smoked glass slide by Worthington [3].

gressed considerably [15, 16]: High-speed imaging methods were digitized [17], drop generators were improved [18-20], and ongoing developments in nanoscale material science allowed for the detailed fabrication and characterization of a variety of target substrates. These recent developments have resulted in major progress regarding the understanding of drop impact [7, 8, 21-36].

The beauty and richness in phenomena observed for the impact of a single drop on a dry solid surface is illustrated in figure 1.2. Capillary oscillations of an impacting microdroplet result in a strong (but smooth) deformation of the droplet surface after impact [37], shown in figure 1.2 (a). Figure 1.2 (b) shows a bubble that is entrapped after the impact a water microdrop [13]. Impact of a hot metal microdrop on a glass surface results in partial evaporation of the glass, which prevents contact between the droplet and the substrate [38]. The droplet spreads into an extremely thin metal sheet, in which holes form as shown in figure 1.2 (c). Figure 1.2 (d) shows the spectacular splash of an ethanol drop of moderate diameter $\left(D_{0} \sim 3 \mathrm{~mm}\right)$ and velocity $\left(V_{0}=3.7 \mathrm{~m} / \mathrm{s}\right)$ impacting on a smooth solid substrate. Similar experiments have revealed that this splash is completely suppressed at lower ambient pressures, which evidences the key role of the surrounding gas [39]. Figure 1.2 (e) illustrates the influence of the substrate morphology: The directional splash observed here is due to a regular pattern of microscopic pillars on the substrate [28]. An example of a nickel droplet that impacts a cold substrate with a velocity $V_{0}=180 \mathrm{~m} / \mathrm{s}$ and subsequently solidified [40] is shown in figure 1.2 (f). Finally, figure $1.2(\mathrm{~g})$ shows the spreading of a drop over a smooth surface. At the edge of the spreading drop a rim is formed, in which spatial oscillations are clearly visible. 
Whether and how these phenomena occur is crucial for understanding a variety of natural phenomena and industrial processes. For example, the impact of rain affects the erosion of soil [41], buildings [42], and wind turbine blades [43, 44]. Industrial applications of droplet impact range from ink-jet printing [18] to cleaning of semiconductor wafers [45] or steel strip [46], and from thermal spraying [38] to fuel fragmentation in engines [47]. Finally, novel technologies depend on highly controlled droplet impact, for instance 3D-printing [48] or deposition of cell-containing drops for the fabrication of living tissue [49-51]. This vast range of applications is reflected by a wide range of droplet diameters and impact velocities shown in figure 1.3 .

However, the vast majority of experimental work has been performed for relatively large $\left(0.5 \mathrm{~mm}<D_{0}<5 \mathrm{~mm}\right)$ and slow $\left(0.1 \mathrm{~m} / \mathrm{s}<V_{0}<10 \mathrm{~m} / \mathrm{s}\right)$ droplets, since these are easy to create by dripping from a needle and subsequently adjusting the height of the needle. Using alternative methods, smaller and faster droplets have been generated, as indicated by the colored data points in figure 1.4. For example, cold spraying [38], droplet-deflection from a droplet train [20], or impact of fast surfaces onto slowly moving droplets [11] have been used to generate fast $\left(V_{0} \gtrsim 50 \mathrm{~m} / \mathrm{s}\right)$ micro-droplet impacts. As these methods are generally highly challenging, most of these works focus on the impact-generation method rather than the physics of droplet impact. The exception to these complex droplet-generation methods is using drop-ondemand systems as used in ink-jet printers, which offer highly reproducible droplet generation. Using ink-jet generated droplets, which have a typical diameter of 30 to $100 \mu \mathrm{m}$ and a velocity of 0.1 to $10 \mathrm{~m} / \mathrm{s}$, some works have focused on the physics of impact [13,37], but here the impact velocities are lower than for most applications. Consequently, details of droplet impact in the small-scale, high-velocity regime are hardly known. This means that several upcoming technologies face delayed introduction and that current industrial processes can be optimized only in part. For example, drop-on-demand printing of pure metals is still not widely introduced, since the ejection and impact dynamics of pure-metal droplets are poorly understood. Another example is bioprinting, in which cell-containing droplets are deposited to construct tissue replacements for damaged or dysfunctional tissues. However, significant cell death still hampers high-throughput cell deposition technologies, which are required to construct tissues of a relevant size.

Therefore, the motivation of this thesis is to enable novel technologies and solutions for long-standing industrial problems by improving our understanding of fast, micro-scale droplet impact. To this aim, key developments in the field of droplet impact will be concisely discussed in the next section. 

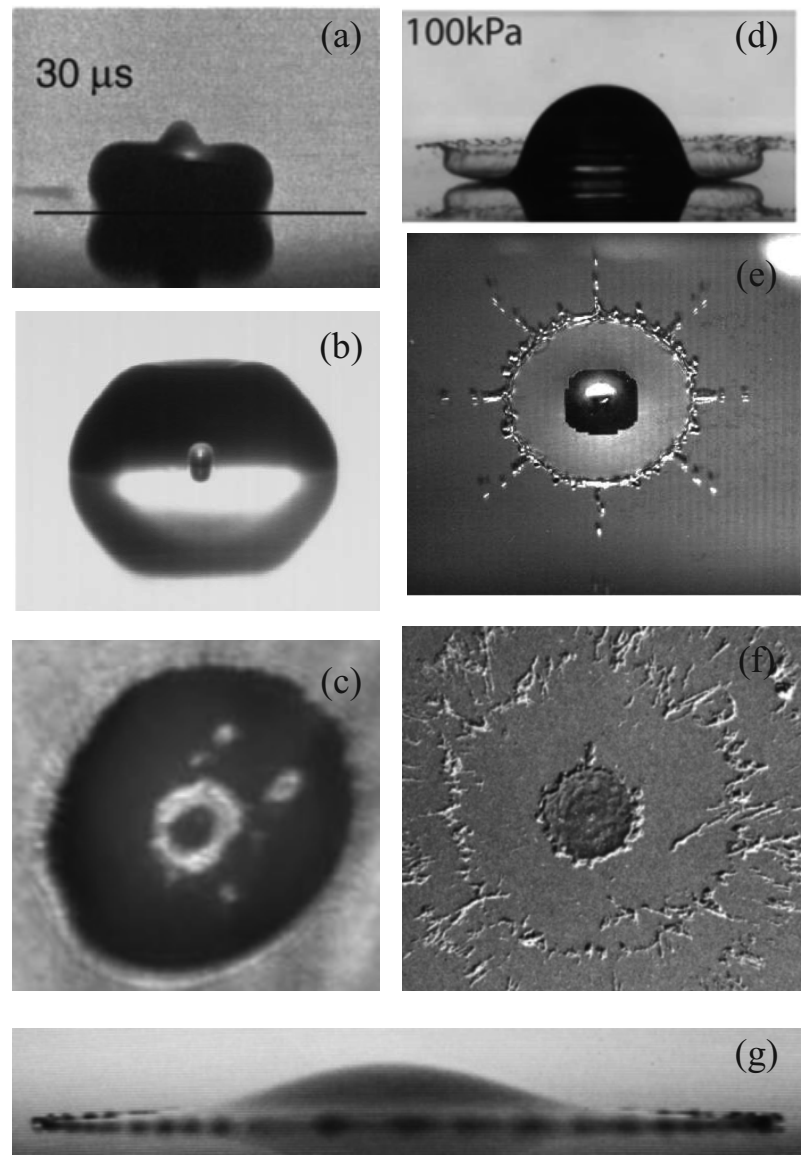

Figure 1.2: Example outcomes of droplet impact, illustrating the wide variety of observed impact behaviors. (a,b,c) Impact of micrometer-sized droplets on solid surface. (a) A water drop impinging on a smooth surface, clearly exhibiting a capillary wave [37]; (b) A water drop with diameter $D_{0}=76 \mu \mathrm{m}$ and impact velocity $V_{0}=3.1 \mathrm{~m} / \mathrm{s}$, after impacting on a smooth substrate [13]. (c) Impact of a hot $(\sim 2000 \mathrm{~K})$ metal microdrop on a glass surface [38]; (d,e) A mm-sized drop with an impact velocity of several meters per second on a (d) smooth [39] and (e) superhydrophobic micro-structured substrate [28]. (f) The solidification pattern after a nickel drop with $D_{0}=200 \mu \mathrm{m}$ and $V_{0}=180 \mathrm{~m} / \mathrm{s}$ impacted on a substrate [40]. (g) Impact of a mm-sized droplet, clearly exhibiting waves on the rim. 


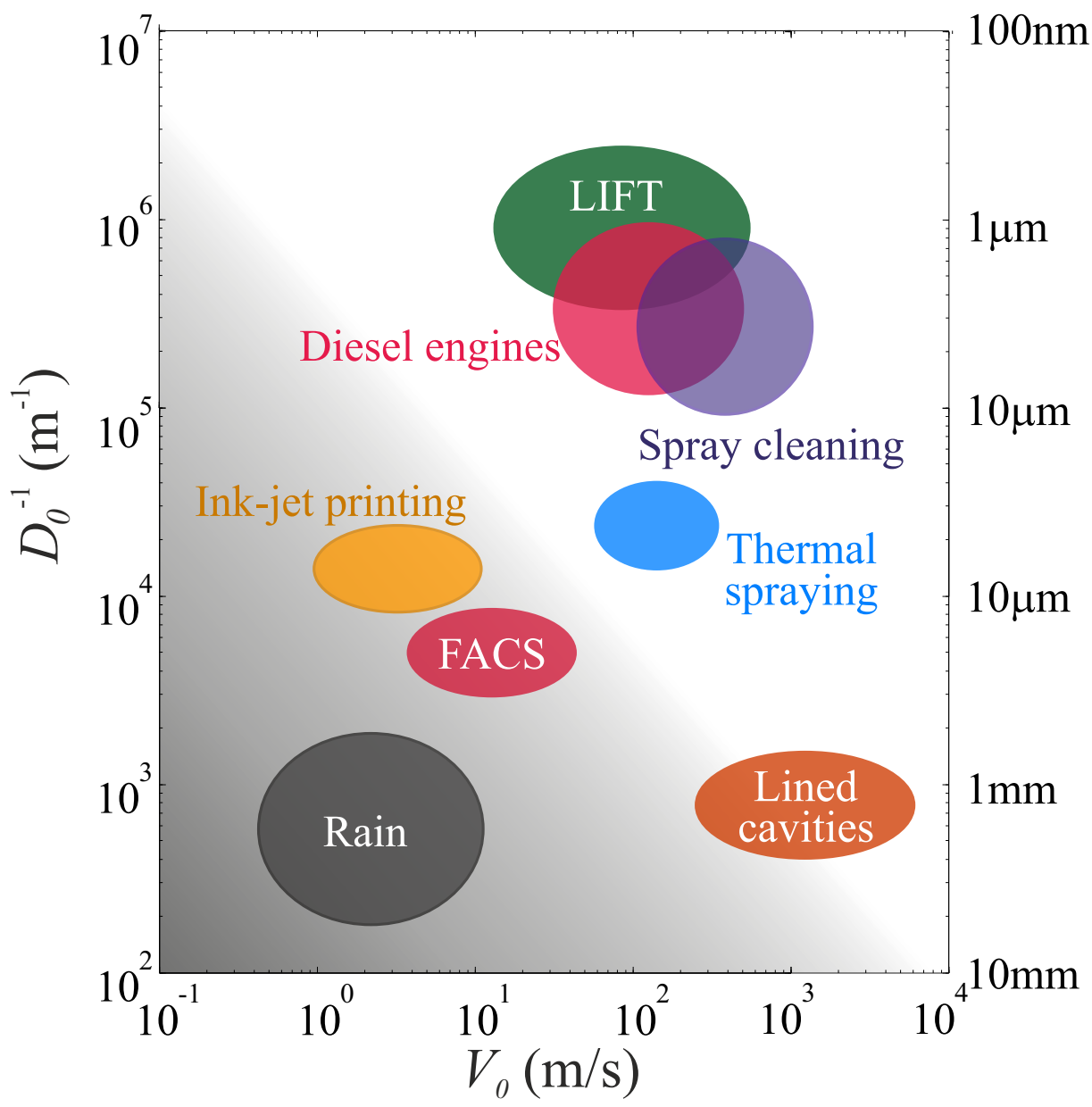

Figure 1.3: Indicative droplet sizes and impact velocities for various applications. LIFT: Laser-induced forward transfer. FACS: Flow-assisted cell cytometry. 


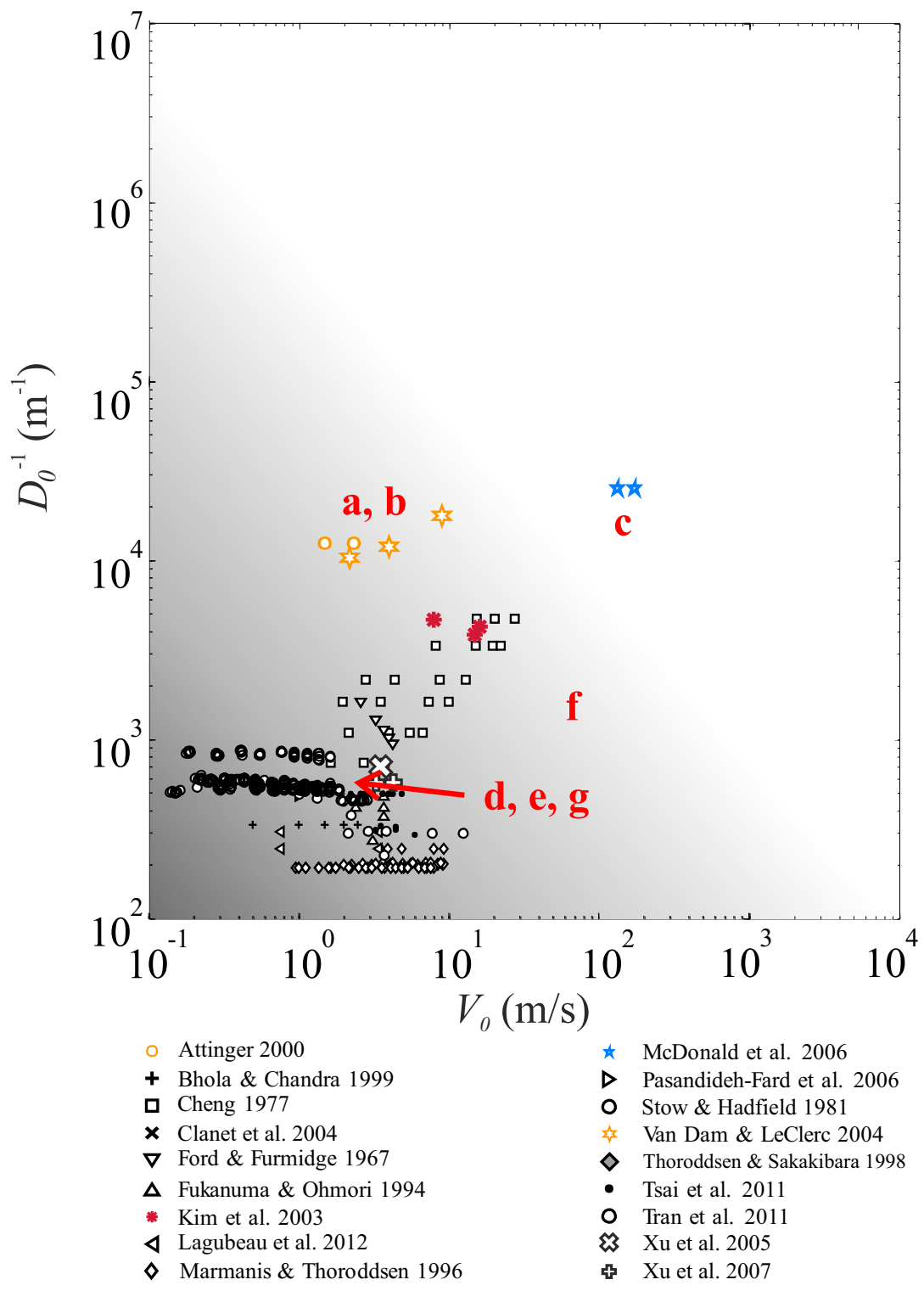

Figure 1.4: Overview of experimental work on droplet impact. The colors indicate the droplet-generation method, and correspond to the applications in figure 1.3. The letters correspond to the impact events shown in figure 1.2. 


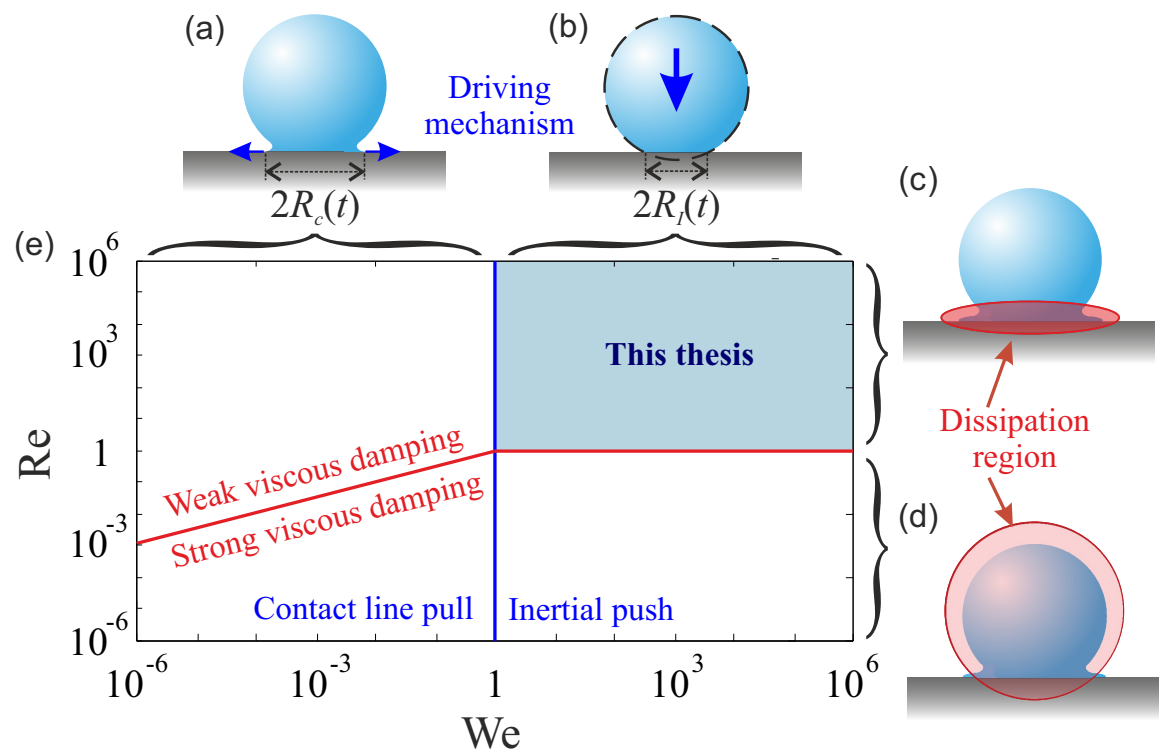

Figure 1.5: Illustration of the main regimes of droplet impact. Figures (a) and (b) illustrate capillary-driven droplet impact and inertia-driven impact, respectively (the arrows illustrate the driving force). Figure (c) illustrates inviscid spreading, in which viscous dissipation mainly occurs close to the surface. Viscous spreading is illustrated in figure (d), where viscosity plays a key role within the entire droplet. Finally, the phase diagram in figure (e) displays the four regimes: the threshold $\mathrm{We}=1$ separates the capillary- from the inertiadriven regimes, as indicated by the solid vertical line. The red line indicates the separation of the viscous and inviscid spreading regimes. 
(a)

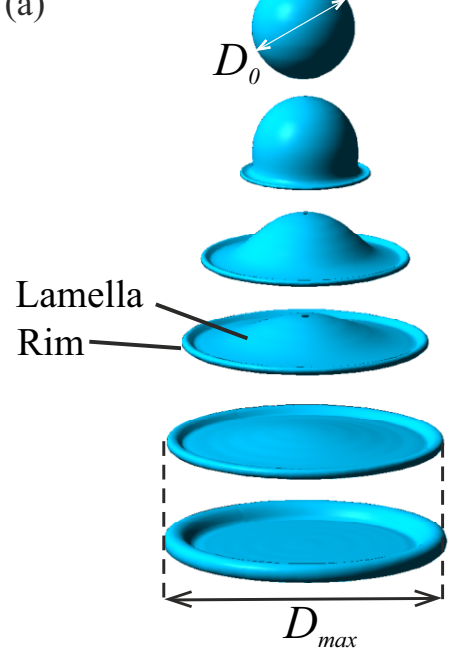

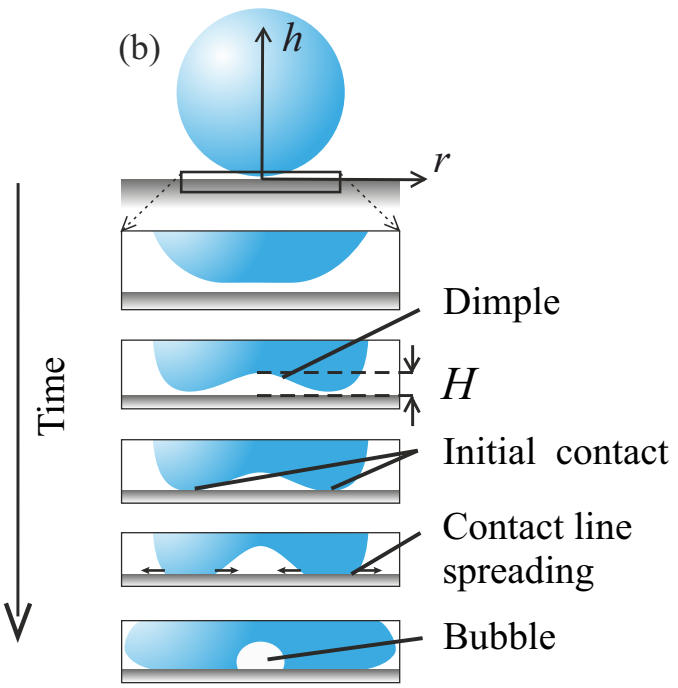

Figure 1.6: Overview of the different stages of droplet impact. (a) A droplet with diameter $D_{0}$ and velocity $V_{0}$ is impacting onto a flat smooth surface (only liquid shown). During its spreading into a lamella, a rim is formed. (b) Droplet-substrate interaction in the initial stage of impact, adapted from [32]. The rectangle in the top image is enlarged in the subsequent images, showing snapshots of the cross-section of the droplet. Initially the droplet flattens and forms a dimple, due to compression of the ambient gas between the droplet and the surface. After the initial contact is made, the air under the dimple is entrained and contracts into a bubble.

\subsection{Droplet impact: Fundamentals and recent developments}

In droplet impact on a smooth solid substrate, various driving- and dissipation mechanisms are encountered. These primarily depend on the impact velocity, the droplet diameter, and the liquid properties. To assess which driving mechanism is dominant, first we consider a droplet approaching the surface at negligible velocity. Starting at the instant of contact, surface tension pulls the contact line away from the initial position of contact (see figure 1.5 (a)). This capillary-driven spreading radius was recently found to scale as $r_{\sigma}(t) \sim\left(\sigma D_{0} / \rho\right)^{1 / 4} \sqrt{t}$, with time $t$, density $\rho$, and surface tension $\sigma$ [52]. providing a capillary spreading velocity $V_{\text {sigma }}(t) \sim\left(\sigma D_{0} / \rho\right)^{1 / 4} t^{-1 / 2}$. For the capillary velocity we have assumed a low-viscosity liquid; for the influence of viscosity see [53].

However, for larger impact velocities, the droplet's inertia increasingly influences the droplet's contact with the surface. In this case, the simplest approach to model the radial position of the contact line of the impacting droplet is to assume that the 
droplet initially maintains its shape and initial velocity, as illustrated by the dashed circle in figure 1.5 (b). The image represents very high impact velocities, in which the droplet's bottom is compressed. Under these assumptions, the radial position of the intersection scales as $r_{I}(t) \sim \sqrt{V_{0} D_{0} t}$. Surprisingly, this scaling is maintained even if compressibility is negligible and the droplet expands at the impact surface, as will be discussed in chapter 4 . The aforementioned scaling provides the inertial spreading velocity $V_{I}(t) \sim \sqrt{V_{0} D_{0} / t}$ with initial diameter $D_{0}$ and impact velocity $V_{0}$. The transition from the capillary- to the inertial-spreading regime takes place if $V_{I}>$ $V_{\sigma}$, yielding a Weber number of $\mathrm{We}=\rho D_{0} V_{0}^{2} / \sigma=1$. This regime transition is indicated by the vertical solid line in figure 1.5 (e).

During impact, the droplet's initial inertial- and surface energy are converted as discussed in ref. [54]. For highly viscous liquids, the impact process is dissipationdominated and strong damping within the full droplet is observed shortly after impact. For the previously discussed inertia-driven impact regime (We $>1$ ), the driving force (inertia) and viscous dissipation are balanced by the Reynolds number $\operatorname{Re}=\rho D_{0} V_{0} / \mu$, with $\mu$ the liquid viscosity. In the inertial regime (We $>1$ and $\operatorname{Re}>1$ ), significant viscous dissipation is encountered only in a region close to the surface as illustrated in figure 1.5 (c). Therefore, the main flow within the droplet during impact can be approximated by ideal flow (i.e. omitting viscosity and surface tension). Still, for describing key parameters such as the maximal spreading of the droplet during impact, viscosity and surface tension have to be included as will be discussed. For viscosity-dominated impact (for which $\operatorname{Re}<1$ ), strong damping within the full droplet is observed as illustrated by figure 1.5 (d), and the ideal-flow approximation breaks down. For the capillary-driven impact regime $(\mathrm{We}<1)$, the dimensionless parameter describing the balance between the driving force (surface tension) to viscous dissipation is the Ohnesorge number: $\mathrm{Oh}=\sqrt{\mathrm{We}} / \mathrm{Re}<1$. For $\mathrm{Oh}>1$, capillary-dominated (essentially inviscid) flow is expected, which for example results in the capillary oscillations shown in figure 1.2 (a). Viscosity-dominated flow is observed for $\mathrm{Oh}<1$ (not shown).

Figure 1.5 (e) provides a phase diagram indicating the dominant driving- and dissipation mechanisms. Since the majority of applications discussion in section 1.1 concerns high Reynolds- and Weber-number impact, and this is also the most complex regime (all impacts shown in figure 1.2 (b) to (g) occur in the inertial regime), the focus of this thesis is inertia-dominated spreading in the inviscid regime. Recently, significant progress has been made especially for three sub-topics of droplet impact on smooth solid surfaces, which are concisely discussed below. 


\subsubsection{Droplet spreading}

During impact, the top of the droplet initially maintains its downward velocity, resulting in an inertial pressure which is highest at the impact surface. Consequently, the liquid at the bottom of the droplet is pressurized and accelerated in the radial direction. This phenomenon, called "spreading" is illustrated in figure 1.6 (a). However, an impacting droplet does not spread forever, but instead reaches some maximum diameter $D_{m}$. Clearly, the drop inertia pushes the liquid outwards, but what limits the spreading? In the literature, this limited spreading has been attributed to either surface tension, which pulls along the extending surface and gives rise to a capillary pressure, or to viscous energy dissipation in the spreading liquid $[6,12,22,23,52,55,56]$.

Initial models for the maximal spreading diameter balance the initial inertial energy of the drop to the surface energy of the flattened drop, or the dissipation within the drop during impact $[6,12,22]$. Although these models sufficiently describe the maximal spreading diameter for certain parts of the impact parameter space, they do not capture the formation of a rim at the edge of the lamella and the formation of a viscous boundary layer growing from the liquid-solid interface. Recent models include these parameters [23, 35, 55-57]. However, the model assumptions are not always met (e.g. the assumption of a space-invariant boundary layer thickness [57]), and validation is incomplete even in the millimeter-scale regime (e.g. experimental scaling relationships for the development of the rim are not yet conclusive [25]). Therefore, the applicability of these droplet spreading models is still a matter of active scientific debate, and new models remain being developed.

\subsubsection{Air film interaction}

Remarkably, the impact of a droplet occurs through a number of subsequent phases starting even before the droplet touches the surface. As illustrated in figure 1.6b, as the drop approaches the solid substrate the air between the falling drop and the substrate is strongly squeezed. This leads to a pressure build-up underneath the drop. Generally, the air underneath the drop cannot escape [6, 25, 30, 32, 58, 59]. As a consequence, the drop does not touch the substrate immediately but instead floats on a thin air film. The pressure build-up underneath the drop leads to a deformation of the drop surface, see figure 1.6 (b). In particular, a dimple forms in the center of the drop [29]. Consequently, touch-down of the drop occurs off-center, at a location where the distance to the substrate is smallest. During the subsequent wetting of the substrate, an air bubble is entrapped [29], of which an example is shown in figure 1.2 (b). Although this entrainment mechanism was identified as early as 1991 [6], the shape of the air film and the size of the entrained bubble was quantitatively investigated 
only recently (as discussed in more detail in appendix A). Whether the deformation of the droplet and the entrainment of air are scalable to $\mu \mathrm{m}$-sized droplet impact is discussed in chapter 4 .

\subsubsection{Splashing}

Splashing is the fragmentation of a drop upon impact, of which a typical example is shown in figure 1.2 (d). It is well known that splashing is observed only beyond certain impact velocities [3]. However, establishing the threshold splash velocity has proven remarkably difficult. Initial quantitative work mainly describe the splash threshold as a function of the (non-dimensional) droplet diameter, impact velocity, and surface roughness $[4,11,47,60]$. Recently however, it has been shown that reducing the pressure of the ambient gas can completely suppress the splash threshold. This discovery has sparked numerical and theoretical attempts to calculate the splash threshold [36, 58, 61, 62], which work well in the mm-sized droplet impact regime. However, a key assumption is the continuity of the thin film of air between the droplet and the surface, which is violated for micrometer-scale impact. As splashing experiments in the micrometer-scale regime are not conclusive [11,47], the splash threshold for these small droplets is unknown.

\subsection{Research problem and scientific approach}

As described, the motivation of this thesis is to enable novel technologies and solutions for long-standing industrial problems by improving our understanding of fast, micro-scale droplet impact. To meet this aim, three research gaps need to be addressed:

1. Creating single, high-speed droplet impacts: As discussed in section 1.1, several methods exist to create single micro-droplet impact at high velocities, but due to their challenging nature they have not been employed to visualize the impact in detail. Therefore, to visualize the impact of such droplets in detail, first a novel, laser-based droplet-generation method is developed (see chapter 2). Subsequently, the impact of these droplets is visualized as described in chapter 3. Next, to improve on these results, an existing method of creating highly reproducible micro-droplet impact is optimized and significantly extended. This allows visualization at unparalleled spatial and temporal resolutions, as described in chapter 4 .

2. Understanding microdroplet impact: A first step in understanding droplet impact is adequate visualization. However, due to the fast impact dynamics, 
sophisticated visualization methods are required. For example, the impact of droplet with diameter $D_{0}=50 \mu \mathrm{m}$ and velocity $V_{0}=50 \mathrm{~m} / \mathrm{s}$ occurs on a typical time scale of $\tau \sim D_{0} / V_{0}=1 \mu \mathrm{s}$, and a frame rate of $\sim 10^{7}$ frames per second is required for observation of the impact dynamics. However, commercial highspeed cameras only allow visualization at such frame rates at relatively low resolutions. Flash illumination offers high-resolution visualization, and was applied to visualize fast micro-droplet impact in unparalleled detail. Second, the dynamics of the flow inside the droplet are only partly known, also for mm-scale droplets. Therefore, to extend our understanding of this flow, and in particular the development of the boundary layer and the rim, numerical modeling is applied. Both the experiments and the simulations are described in chapter 4.

3. Demonstrating novel applications of micro-droplet impact: Two novel applications of micro-droplet impact are demonstrated in this thesis. First, metal structures have hardly been 3D-printed despite their beneficial material properties for some applications. The key reason is that melting, ejecting, and depositing common metals (e.g. copper) is highly challenging using conventional techniques. Here, using a method called laser-induced forward transfer, we create and deposit metal micro-droplets to construct metal pillars as described in chapters 5 and 6 , respectively.

Second, the manufacturing of cell-containing tissue constructs or even complete organs and subsequent transplantation to patients has been demonstrated. However, the biofabrication of such tissues remains challenging, since conflicting requirements exist for the deposition of the cells. Ideally, high-viscosity, cell-containing liquids or gels are deposited on a specific spatial location on a surface to which the cells sufficiently adhere. Obviously, the viability of the cells should be maintained during the deposition process. In chapter 7, we quantify the relationship between the cell-containing liquid, the deposition process, and the cell viability after deposition in spray bioprinting. We argue that cell spray systems may allow to achieve all aforementioned requirements for cell deposition. Finally, to assess the adhesion of cells to a surface, we expose a cell monolayer to a shear force generated by a submerged jet (of which the flow is related to droplet impact). Since the shear stress for this configuration is known, the cell adhesion strength is calculated and the detachment regime is visualized (chapter 8). 


\subsection{Reading guide}

The structure of the thesis follows the research gaps identified in section 1.3, which are addressed in chapters 2 to 8 of this thesis. Finally, in chapter 9 , the results are summarized and an outlook is presented. As the thesis is paper-based, some overlap exists between this chapter and the introduction of the individual chapters, and similarities exist between some figures. Since most chapters are the result of fruitful and enjoyable collaborations with researchers within and outside the Physics of Fluids group, the author's contributions are indicated as a footnote on the title page of the chapters. Finally, significant additional scientific results have been achieved within this $\mathrm{PhD}$ project [63-67], but are not included in this thesis to maintain a clear scope.

\section{References}

[1] L. Da Vinci, Codex Leicester, The notebooks of Leonardo da Vinci (1508).

[2] E. Villermaux and B. Bossa, Drop fragmentation on impact, Journal of Fluid Mechanics 668, 412 (2011).

[3] A. Worthington, On the forms assumed by drops of liquids falling vertically on a horizontal plate, Proceedings of the Royal Society of London. 25, 261 (1876).

[4] C. D. Stow and M. G. Hadfield, An experimental investigation of fluid flow resulting from the impact of a water drop with an unyielding dry surface, Proceedings of the Royal Society A: Mathematical, Physical and Engineering Sciences 373, 419 (1981).

[5] L. Wachters and N. Westerling, The heat transfer from a hot wall to impinging water drops in the spheroidal state, Chemical Engineering Science 21, 1047 (1966).

[6] S. Chandra and C. T. Avedisian, On the collision of a droplet with a solid surface, Proceedings of the Royal Society A: Mathematical, Physical and Engineering Sciences 432, 13 (1991).

[7] T. Tran, H. J. J. Staat, A. Prosperetti, C. Sun, and D. Lohse, Drop impact on superheated surfaces, Physical Review Letters 108, 36101 (2012).

[8] T. Tran, H. J. J. Staat, A. Susarrey-Arce, T. C. Foertsch, A. van Houselt, H. J. G. E. Gardeniers, A. Prosperetti, D. Lohse, and C. Sun, Droplet impact on superheated micro-structured surfaces, Soft Matter 9, 3272 (2013).

[9] J. Dear and J. Field, Highspeed photography of surface geometry effects in liquid/solid impact, Journal of applied physics 63, 1015 (1988).

[10] J. Field, J. Dear, and J. Ogren, The effects of target compliance on liquid drop impact, Journal of Applied Physics 65, 533 (1989).

[11] C. Mundo, M. Sommerfeld, and C. Tropea, Droplet-wall collisions: Experimental studies of the deformation and breakup process, International Journal of Multiphase Flow 21, 151 (1995).

[12] L. Cheng, Dynamic spreading of drops impacting onto a solid surface, Industrial \& Engineering Chemistry Process Design Development 16, 192 (1977).

[13] D. B. van Dam and C. Le Clerc, Experimental study of the impact of an ink-jet printed droplet on a solid substrate, Physics of Fluids 16, 3403 (2004). 
[14] M. Rein, Phenomena of liquid drop impact on solid and liquid surfaces, Fluid Dynamics Research 12, 61 (1993).

[15] A. Yarin, Drop impact dynamics: splashing, spreading, receding, bouncing, Annu. Rev. Fluid Mech. 38, 159 (2006).

[16] A. L. Moreira, A. Moita, and S. Chandra, Handbook of Atomization and Sprays (Springer, Dordrecht, The Netherlands, 2011), pp. 183-211.

[17] M. Versluis, High-speed imaging in fluids, Experiments in Fluids 54, 1458 (2013).

[18] H. Wijshoff, The dynamics of the piezo inkjet printhead operation, Physics Reports 491, 77 (2010).

[19] P. Ben-Tzvi and W. Rone, Microdroplet generation in gaseous and liquid environments, Microsystem Technologies 16, 333 (2009).

[20] P. E. Frommhold, A. Lippert, F. L. Holsteyns, and R. Mettin, High-speed monodisperse droplet generation by ultrasonically controlled micro-jet breakup, Experiments in Fluids 55, 1716 (2014).

[21] D. Richard, C. Clanet, and D. Quéré, Contact time of a bouncing drop, Nature 417, 811 (2002).

[22] C. Clanet, C. Béguin, D. Richard, and D. Quéré, Maximal deformation of an impacting drop, Journal of Fluid Mechanics 517, 199 (2004).

[23] J. Eggers, M. a. Fontelos, C. Josserand, and S. Zaleski, Drop dynamics after impact on a solid wall: Theory and simulations, Physics of Fluids 22, 062101 (2010).

[24] M. Reyssat, D. Richard, C. Clanet, and D. Quéré, Dynamical superhydrophobicity, Faraday Discussions 146, 19 (2010).

[25] J. de Ruiter, R. E. Pepper, and H. a. Stone, Thickness of the rim of an expanding lamella near the splash threshold, Physics of Fluids 22, 022104 (2010).

[26] P. Tsai, S. Pacheco, C. Pirat, L. Lefferts, and D. Lohse, Drop impact upon micro- and nanostructured superhydrophobic surfaces., Langmuir : the ACS journal of surfaces and colloids 25, 12293 (2009).

[27] P. Tsai, R. C. A. van der Veen, M. van de Raa, and D. Lohse, How micropatterns and air pressure affect splashing on surfaces., Langmuir 26, 16090 (2010).

[28] P. Tsai, M. H. W. Hendrix, R. R. M. Dijkstra, L. Shui, and D. Lohse, Microscopic structure influencing macroscopic splash at high Weber number, Soft Matter 7, 11325 (2011).

[29] W. Bouwhuis, R. C. A. van der Veen, T. Tran, D. L. Keij, K. G. Winkels, I. R. Peters, D. van der Meer, C. Sun, J. H. Snoeijer, and D. Lohse, Maximal air bubble entrainment at liquid-drop impact, Physical Review Letters 109, 264501 (2012).

[30] J. M. Kolinski, S. M. Rubinstein, S. Mandre, M. P. Brenner, D. A. Weitz, and L. Mahadevan, Skating on a film of air: drops impacting on a surface, Physical Review Letters 108, 074503 (2012).

[31] J. de Ruiter, J. M. Oh, D. van den Ende, and F. Mugele, Dynamics of collapse of air films in drop impact, Physical Review Letters 108, 074505 (2012).

[32] R. C. A. van der Veen, T. Tran, D. Lohse, and C. Sun, Direct measurements of air layer profiles under impacting droplets using high-speed color interferometry, Physical Review E 85, 026315 (2012).

[33] C. W. Visser, Y. Tagawa, C. Sun, and D. Lohse, Microdroplet impact at very high velocity, Soft Matter 8, 10732 (2012).

[34] D. Quéré, Leidenfrost Dynamics, Annual Review of Fluid Mechanics 45, 197 (2013).

[35] H. Lastakowski, F. Boyer, a. L. Biance, C. Pirat, and C. Ybert, Bridging local to global dynamics 
of drop impact onto solid substrates, Journal of Fluid Mechanics 747, 103 (2014).

[36] G. Riboux and J. Gordillo, The critical impact speed for the splash of a drop, arXiv preprint arXiv:1401.6943 1 (2014).

[37] D. Attinger, Z. Zhao, and D. Poulikakos, An experimental study of molten microdroplet surface deposition and solidification: Transient behavior and wetting angle dynamics, Journal of Heat Transfer 122, 544 (2000).

[38] A. McDonald, M. Lamontagne, C. Moreau, and S. Chandra, Impact of plasma-sprayed metal particles on hot and cold glass surfaces, Thin Solid Films 514, 212 (2006).

[39] L. Xu, W. Zhang, and S. Nagel, Drop splashing on a dry smooth surface, Physical Review Letters 94, 184505 (2005).

[40] K. Haller and Y. Ventikos, Computational study of high-speed liquid droplet impact, Journal of Applied Physics 92, 2821 (2002).

[41] S. Ahn, S. H. Doerr, P. Douglas, R. Bryant, C. A. Hamlett, G. McHale, M. I. Newton, and N. J. Shirtcliffe, Effects of hydrophobicity on splash erosion of model soil particles by a single water drop impact, Earth Surface Processes and Landforms 38, 1225 (2013).

[42] A. Erkal, D. DAyala, and L. Sequeira, Assessment of wind-driven rain impact, related surface erosion and surface strength reduction of historic building materials, Building and Environment 57, 336 (2012).

[43] M. Keegan, D. Nash, and M. Stack, in Proceedings of the TURBO EXPO (ASME, Copenhagen, Denmark, 2012), pp. 1-12.

[44] J. Field, J.-J. Camus, M. Tinguely, D. Obreschkow, and M. Farhat, Cavitation in impacted drops and jets and the effect on erosion damage thresholds, Wear 290-291, 154 (2012).

[45] H. F. Okorn-Schmidt, F. Holsteyns, a. Lippert, D. Mui, M. Kawaguchi, C. Lechner, P. E. Frommhold, T. Nowak, F. Reuter, M. B. Pique, C. Cairos, and R. Mettin, Particle Cleaning Technologies to Meet Advanced Semiconductor Device Process Requirements, ECS Journal of Solid State Science and Technology 3, N3069 (2013).

[46] W. Melfo, H. Bolt, M. Rijnders, D. Staalman, C. B. Castro, D. Crowther, and B. Jana, Experimental Study on Primary Scale Formation and Descalability on Steels Containing $\mathrm{Ni}$ and $\mathrm{Ni}+\mathrm{Si}$, ISIJ International 53, 866 (2013).

[47] A. Moreira, A. Moita, and M. Panão, Advances and challenges in explaining fuel spray impingement: How much of single droplet impact research is useful?, Progress in Energy and Combustion Science 36, 554 (2010).

[48] C. Ru, J. Luo, S. Xie, and Y. Sun, A review of non-contact micro- and nano-printing technologies, Journal of Micromechanics and Microengineering 24, 053001 (2014).

[49] B. Derby, Bioprinting: inkjet printing proteins and hybrid cell-containing materials and structures, Journal of Materials Chemistry 18, 5717 (2008).

[50] S. Tasoglu and U. Demirci, Bioprinting for stem cell research., Trends in Biotechnology 31, 10 (2013).

[51] K. W. Binder, A. J. Allen, J. J. Yoo, and A. Atala, Drop-on-demand ink-jet bioprinting: a primer, Gene Therapy and Regulation 06, 33 (2011).

[52] A.-L. Biance, C. Clanet, and D. Quéré, First steps in the spreading of a liquid droplet, Physical Review E 69, 016301 (2004).

[53] A. Eddi, K. G. Winkels, and J. H. Snoeijer, Short time dynamics of viscous drop spreading, Physics of Fluids 25, 013102 (2013). 
[54] S. Schiaffino and A. a. Sonin, Molten droplet deposition and solidification at low Weber numbers, Physics of Fluids 9, 3172 (1997).

[55] M. Pasandideh-Fard, Y. M. Qiao, S. Chandra, and J. Mostaghimi, Capillary effects during droplet impact on a solid surface, Physics of Fluids 8, 650 (1996).

[56] I. V. Roisman, R. Rioboo, and C. Tropea, Normal impact of a liquid drop on a dry surface: model for spreading and receding, Proceedings of the Royal Society A: Mathematical, Physical and Engineering Sciences 458, 1411 (2002).

[57] S. Tabakova, F. Feuillebois, A. Mongruel, V. Daru, and S. Radev, First stages of drop impact on a dry surface: asymptotic model, Zeitschrift für angewandte Mathematik und Physik 63, 313 (2012).

[58] S. Mandre, M. Mani, and M. P. Brenner, Precursors to splashing of liquid droplets on a solid surface., Physical Review Letters 102, 134502 (2009).

[59] M. M. Driscoll and S. R. Nagel, Ultrafast Interference Imaging of Air in Splashing Dynamics, Physical Review Letters 107, 154502 (2011).

[60] R. L. VanderWal, G. M. Berger, and S. D. Mozes, The combined influence of a rough surface and thin fluid film upon the splashing threshold and splash dynamics of a droplet impacting onto them, Experiments in Fluids 40, 23 (2005).

[61] M. Mani, S. Mandre, and M. Brenner, Events before droplet splashing on a solid surface, Journal of Fluid Mechanics 647, 163 (2010).

[62] S. Mandre and M. Brenner, The mechanism of a splash on a dry solid surface, Journal of Fluid Mechanics 690, 148 (2012).

[63] E. Karatay, A. S. Haase, C. W. Visser, C. Sun, D. Lohse, P. A. Tsai, and R. G. H. Lammertink, Control of slippage with tunable bubble mattresses., Proceedings of the National Academy of Sciences of the United States of America 110, 8422 (2013).

[64] C. W. Visser, Jet injection technology overview, 2013.

[65] R. Pohl, C. W. Visser, G. R. B. E. Römer, C. Sun, A. J. Huis in 't Veld, and D. Lohse, in Proceedings of LPM (LPM, Vilnius, Lithuania, 2014), pp. 1-5.

[66] R. Pohl, C. W. Visser, G. R. B. E. Römer, C. Sun, A. J. Huis in 't Veld, and D. Lohse, in Laser Applications in Microelectronic and Optoelectronic Manufacturing XIX, edited by Y. Nakata, X. Xu, S. Roth, and B. Neuenschwander (SPIE, San Francisco, CA, USA, 2014), p. 89670X.

[67] A. J. Huis in 't Veld, M. B. Hoppenbrouwers, M. Giesbers, R. Pohl, G. R. B. E. Römer, C. W. Visser, C. Sun, and D. Lohse, in Proceedings of the 10th International Conference on MultiMaterial Micro Manufacture (Research Publishing Services, Singapore, 2013), pp. 160-163. 


\section{2 \\ On highly focused supersonic microjets * ${ }^{\dagger}$}

The development of needle-free drug injection systems is of great importance to global healthcare and requires highly-focused microjets with ultra-high velocities (more than 200 $\mathrm{m} / \mathrm{s}$ ) and good reproducibility. We demonstrate a novel method of creating microjets with a very sharp geometry and controlled velocities even for supersonic speeds up to $850 \mathrm{~m} / \mathrm{s}$, going beyond conventional methods with diffuse jets. The microjet is generated by focusing a laser pulse in a liquid-filled glass-microcapillary; a small mass of liquid is instantaneously vaporized, which leads to a shock wave that travels towards the concave free surface where it generates a high-speed microjet. For optimization of the microjet, we conduct a parametric study of the jet velocity, discuss the physical background of these results, and arrive at scaling relations for the jet velocity as function of the various parameters. The focused shape, good controllability, and the high velocities of our microjet constitute a significant step towards the development of reliable needle-free injections.

*Published as: Y. Tagawa, N. Oudalov, C.W. Visser, I.R. Peters, D. van der Meer, A. Prosperetti, C. Sun, D. Lohse, On highly focused supersonic microjets, Physical Review X 2 (3), 031002 (2012).

${ }^{\dagger}$ Experimental setup (in part), experiments (in part) and geometrical optics (in full) by CWV. 


\subsection{Introduction}

Liquid jets have been studied from various perspectives due to their widespread application and the fundamental interest in them (see e.g. the review article by Eggers and Villermaux [1]). Common examples include ink-jet printing [2-4], ultrasound surface cleaning [5-7], the damage done by imploding cavitation bubbles [8], and collapsing voids created through impacts [9-13]. However, all these jets either do not have particular high velocities (i.e. they are at most of the order of $10 \mathrm{~m} / \mathrm{s}$ ) or they are rather uncontrolled.

For the particular application of liquid jets for needle-free drug injection [14], both of these properties - ultra-high velocities and good reproducibility - are however essential, as the aim of this method is to jet a liquid solution containing the drug into human or animal tissue through the skin.

The development of needle-free drug injection systems is of paramount importance to the global fight against the spread of disease. These systems prevent any contamination to human bodies caused by needles. For this last purpose, the important requirements for these microjets are: ultra-high speed (more than $200 \mathrm{~m} / \mathrm{s}$ ), good controllability, fine scale (down to $30 \mu \mathrm{m}$ ) with a highly-focused geometry, and efficiency. Several methods of microjet creation have been previously studied $([16,17])$. However, the shape of these jets is diffusive and the jetting requires a lot of energy. Another problem of conventional methods is that due to the small size of the nozzle diameter, it can easily get clogged, causing disruptions to controllability.

In this study, we present a unique method for the creation of high-speed focused microjets. The microjet has extraordinary characteristics that satisfy the requirements described above: the maximum velocity of the microjet reaches $850 \mathrm{~m} / \mathrm{s}$, i.e. more than a Mach number of 2 . This is much faster than the velocity which can be achieved with conventional techniques. Moreover, the generation of our microjets requires very low laser energies that are comparable to the energy of a laser pointer, i.e. $\approx 10 \mu \mathrm{J}$. The required energy thus is far smaller than for the conventional method proposed by Han \& Yoh [16]. The shape of our microjet is highly-focused and axisymmetric - another feature that is absent from conventional jetting devices. Note that the diameter of the microjet is 5 to 10 times smaller than the diameter of the capillary tube and is decoupled from it. The problem of clogging has rarely occurred when using this method.

To create our supersonic microjets, a concave liquid-air interface is impacted by the shock wave created from the abrupt vaporization of a small mass of liquid caused by the absorption of a laser pulse. The idea of using concave surfaces for jet creation is similar to a so-called 'shaped-charge', although the mechanism of impacting a shock wave on an interface for this purpose has not been reported before. The early 
history of 'shaped-charges' is described by Birkhoff et al. [18]; more recent works are found in Curtis and Kelly [19], Petit et al. [20]. Unlike our work, these authors do not pursue controllability of the high velocity jets. The principle employed in our device is similar to the ones used for the so-called tubular jet, where a meniscus is accelerated into an evacuated tube forming a jet [21, 22], and the jets developing in a suddenly decelerating falling partially liquid-filled tube [23]. However, in both of these methods the jet size is of the order of millimeters and the velocities are only up to $10 \mathrm{~m} / \mathrm{s}$, thus limiting the field of application.

To overcome these limitations, in the present study the meniscus of a liquidfilled microcapillary is accelerated through a powerful and reproducible shock wave, which is generated by laser-induced liquid vaporization $(\S 2.2)$. This gives rise to well-defined jets with diameters well below $20 \mu \mathrm{m}$ and supersonic velocities (§ 2.3). Further questions we want to answer in this study are: Which physical quantities affect the dynamics of the microjet and how to account for the found dependences? How fast can the microjet be? These quantitative dependences of the jet speed on the various control parameters are discussed in $\S 2.4$, culminating in a scaling law for the jet velocity as function of the various parameters. The paper ends with conclusions in $\S 2.5$.

\subsection{Experimental setup and control parameters}

Figure 2.1(a) shows a sketch of the whole experimental setup, which is similar to that used by Sun et al. [24]. One end of the capillary tube is connected to a syringe pump (Model PHD 2000, Harvard Apparatus, USA) containing a water-based red dye. The other end is open to the air. A $532 \mathrm{~nm}, 6 \mathrm{~ns}$ laser pulse $(100 \mathrm{~mJ}$ Nd:YAG laser, Solo PIV, New Wave, USA) is focused through a $10 \times$ microscope objective onto a point of the capillary. The laser energy is monitored by means of an energy meter (Genteceo XLE4 or Gentec-eo QE12SP-S-MT-D0, Canada) placed behind the capillary. The energy absorbed by the working fluid was calibrated by measuring the difference between the readings of the meter with the glass tube filled with the working fluid and with clear water. The jet formation was recorded using high-speed cameras with a frame rate of up to $10^{6} \mathrm{fps}$ (HPV-1, Shimadzu Corporation, Japan and SA1.1 and Photron, USA). The minimum inter-frame and exposure times were $1 \mu \mathrm{s}$ and $250 \mathrm{~ns}$ respectively. This system enabled us to observe the capillary from the bottom using a microscope (Axiovert 40 CFL, Carl Zeiss, Germany). A long-distance microscope (Model K2, Infinity, USA) with a maximum magnification of $12 \times$ was connected to the camera in order to capture the jet formation from the side and vary the field of view by adjusting the magnification. Illumination for the camera was provided 

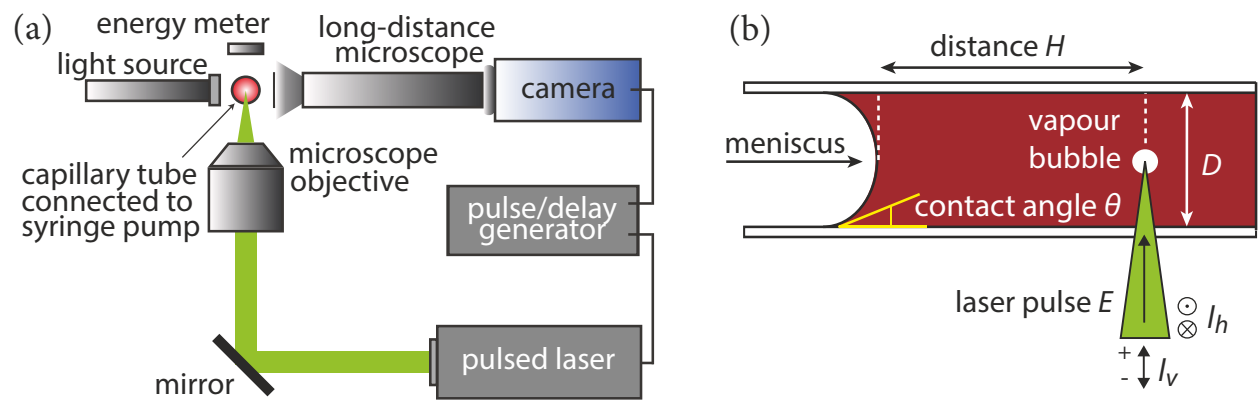

Figure 2.1: (a) A sketch of the experimental setup. The capillary tube is aligned perpendicular to the paper. (b) All experimental parameters with a side view of the capillary in which the microjet formation takes place.

by a fibre lamp (ILP-1, Olympus, Japan) emitting light that passed through the filter protecting the camera. A digital delay generator (Model 555, Berkeley Nucleonics Corporation, USA) was used to synchronise the camera and the laser. Images were analysed with tracking software.

The experimental parameters are indicated in figure 2.1(b) together with a side view of the capillary. The distance between the meniscus and the laser focus position is $H, E$ is the absorbed laser energy, $\theta$ is the contact angle of the liquid with the glass capillary with diameter $D ; l_{v}$ is the distance offset of the laser focus with respect to the capillary axis in the vertical plane, and $l_{h}$ is the focus offset of the laser in the horizontal plane. Three different borosilicate glass capillary tubes (Capillary Tube Supplies Limited, UK) were used in the experiments, with inner diameters $D$ of 50 , 200 and $500 \mu \mathrm{m}$ and outer diameters of 80,220 and $520 \mu \mathrm{m}$, respectively. The inner surface of the tip of the capillary tube was dipped in a hydrophobic solution $(1 \mathrm{H}, 1 \mathrm{H}$, $2 \mathrm{H}, 2 \mathrm{H}$-Perfluorooctyltrichlorosilane), to vary the contact angle $\theta$ between $20^{\circ}$ and $90^{\circ}$. For uncoated tubes, the contact angle was found to be $25^{\circ} \pm 3^{\circ}$, which is similar to the data reported by Sumner et al. [25] for borosilicate glass. The range of the control parameters is summarised in table I.

\subsection{Jet formation and evolution}

An example of such a supersonic jet obtained using the technique of dual-flash illumination by laser-induced fluorescence described in van der Bos et al. [26] is shown in figure 2.2. The capillary is on the right, the jet tip is on the left, and the jet travels from right to left with a speed of $490 \mathrm{~m} / \mathrm{s}$. The tip of this jet has grown into an almost spherical mass which will eventually detach as a droplet. At the other end, the jet is 
Table 2.1: The range of control parameters for the contact angle of the liquid with the glass capillary $\theta$, the distance between the meniscus and the laser focus position $H$, the absorbed laser energy $E$, the focus offset of the laser in the vertical plane $l_{v}$, and the focus offset of the laser in the horizontal plane $l_{h}$. The inner diameters of capillary tubes $D$ are 50,200 , and $500 \mu \mathrm{m}$.

\begin{tabular}{|c|c|c|c|c|c|c|}
\hline & $\theta$ & $E(\mu \mathrm{J})$ & $H(\mu \mathrm{m})$ & $D(\mu \mathrm{m})$ & $l_{v}(\mathrm{~mm})$ & $l_{h}(\mathrm{~mm})$ \\
\hline Lower limit & $20^{\circ}$ & 19 & 200 & 50 & -1 & 0 \\
Upper limit & $90^{\circ}$ & 880 & 2500 & 500 & 2.5 & 0.1 \\
\hline
\end{tabular}

thicker and slower.

High-speed cameras were used to capture the subsequent dynamics of the jet. Figure 2.3 shows several frames of side view images of the jet in the $500 \mu \mathrm{m}$ tube taken with the Shimadzu camera. The open side of the tube is on the left; the other side of the tube is connected to the syringe pump. The bubble is formed at the lower wall of the capillary and mainly expands in the direction of the open end. In spite of off-centered position of the bubble and the asymmetry of the bubble growth, the jet maintains an axisymmetric shape with a sharp tip. The diameter of the jet is always much smaller than that of the capillary. In this instance, the diameter of the jet is $\sim 50 \mu \mathrm{m}$, about 10 times smaller than that of the capillary. The start of the instability which eventually leads to droplet pinch off can be discerned. We have always found that, unless $H \leq D$, the asymmetry of bubble growth does not affect the axisymmetric shape of the jet. This finding supports the hypothesis that the cause of the thin jet is the sudden onset of a high pressure and consequent shock wave rather than the bubble expansion per se.

The left frame of figure 2.4 shows another typical sequence of the jet evolution. The corresponding history of the jet tip velocity is shown in the right frame and it is seen to be non-monotonic. Immediately after the laser pulse, the interface sets into motion (i) and reaches a maximum velocity $V_{\max }$ at (ii), by which time the meniscus has lost the initial concave shape. The subsequent deceleration (iii) is due to the effect of surface tension which retards the motion. As the drop at the jet tip starts to form, the retarding effect of surface tension becomes negligible and the velocity reaches an asymptotic value $V_{j}$ (iv). Eventually, the microdrop detaches from the jet tip. The maximum velocity always occurs at a configuration as shown in image (ii). 
$50 \mu \mathrm{m}$
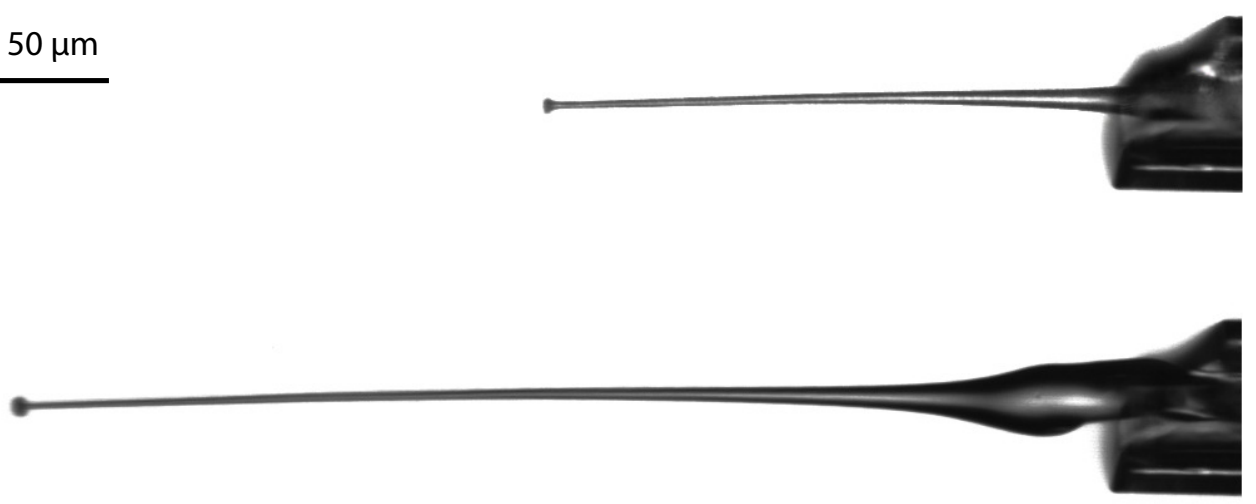

Figure 2.2: Images of a supersonic microjet generated in a $50 \mu \mathrm{m}$ capillary tube. The capillary is visible on the right side, the jet tip is shown on the left. The jet travels from right to left with a speed of $490 \mathrm{~m} / \mathrm{s}$. Time between images is $500 \mathrm{~ns}$.
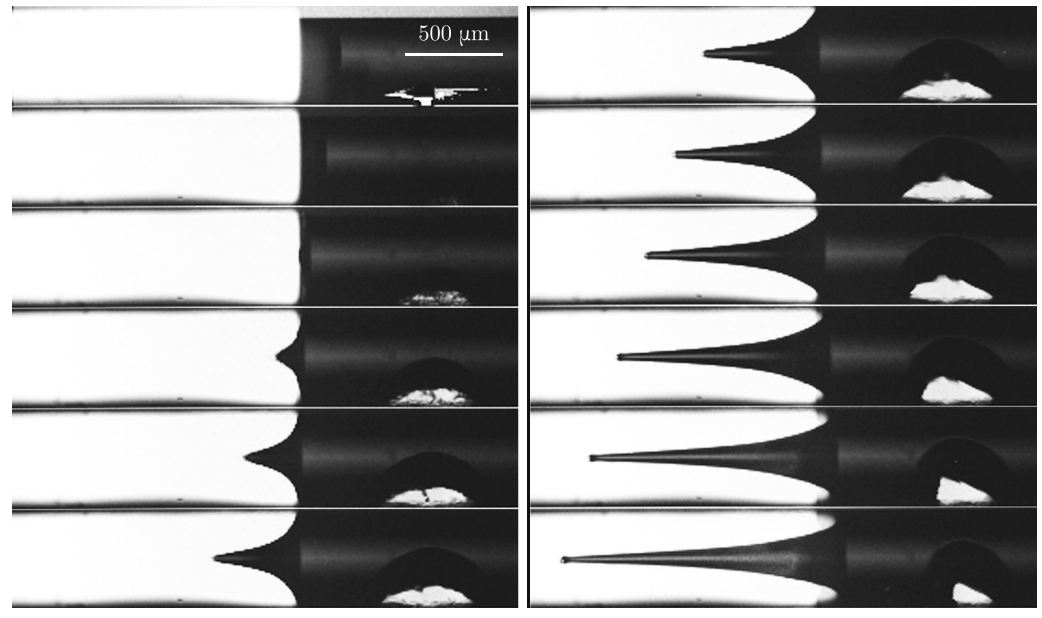

Figure 2.3: Bubble growth and jet evolution after focusing the laser in a $500 \mu \mathrm{m}$ capillary tube. The first image shows the tube when the laser is shot. The subsequent images are taken $7 \mu$ s apart. (The movie is available as supplemental material [figure3.mov] ) 


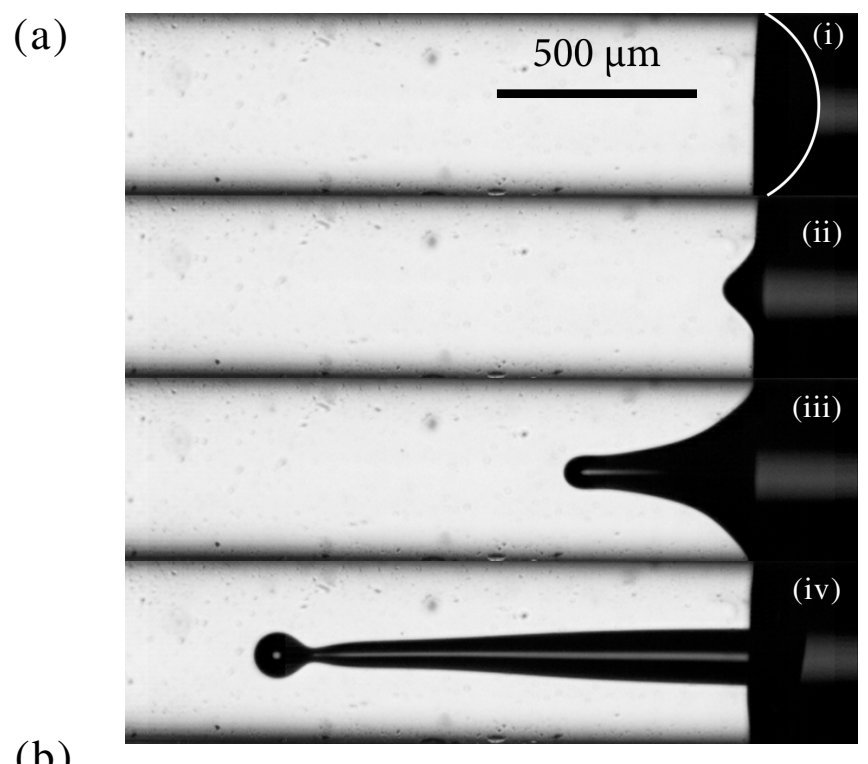

(b)

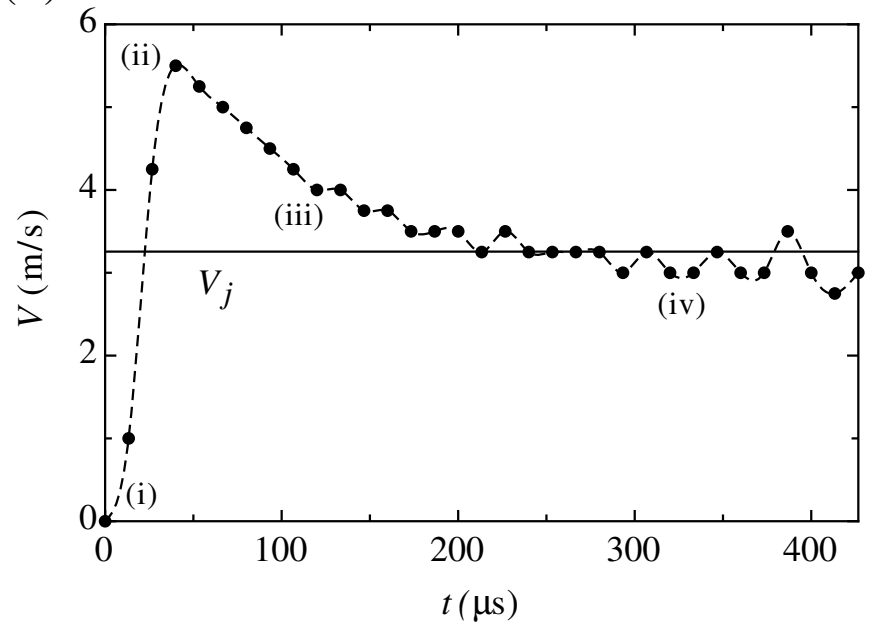

Figure 2.4: (a) Jet evolution after focusing the laser in a $500 \mu \mathrm{m}$ capillary tube. The first image shows the tube at the moment that the laser is shot. The subsequent images are taken at later stages, each corresponding to a point in time shown in the adjacent figure. (b) Velocity of the jet tip in a $500 \mu \mathrm{m}$ tube as a function of time after the laser is shot. 


\subsection{Parameter dependence}

In this section, we study the dependence of the jet speed on various control parameters.

\subsubsection{Contact angle $\theta$}

Since the meniscus shape is crucial for the jet formation, the first parameter we investigated is the contact angle $\theta$ of the liquid with the glass wall of the tube as indicated in figure 2.1(b). The contact angle was calculated by fitting a circle to the free surface of the liquid, measuring the radius of this circle and applying the formula $\cos \theta=D /(2 R)$ which is valid because the effect of gravity in this system is negligible as shown by the smallness of the Bond number Bo $=\rho g R^{2} / \gamma$.

The contact angle is varied by minor adjustments of the liquid volume by means of the syringe pump. The energy and the distance $H$ between focus position and meniscus are kept constant at $460 \pm 20 \mu \mathrm{J}$ and $460 \pm 40 \mu \mathrm{m}$, respectively. Figure 2.5 shows four sequences taken at different initial contact angles, with three snapshots per sequence. The shape of the jet is significantly influenced by the contact angle. These results show that the more curved the meniscus shape (lower contact angle and smaller radius of curvature), the higher the jet velocity due to the increased focusing. In contrast, for increasing contact angles, the jet becomes thicker and less focused. Eventually, when the contact angle is larger than 90 degrees, the focusing is lost, no jet is formed and the liquid is only pushed out as a plug by the expanding bubble.

The parameters that may be expected to play a role in the flow focusing are the initial velocity $V_{0}$ instantaneously acquired by the meniscus and the curvature of the interface given by

$$
\kappa=\frac{4 \cos \theta}{D} .
$$

Dimensional analysis gives us the following scaling for the acceleration of the free surface:

$$
a \propto V_{0}^{2} \frac{\cos \theta}{D} .
$$

The focusing time scale $\Delta t$ is provided by the typical velocity $V_{0}$ and length scale $D$ :

$$
\Delta t \sim \frac{D}{V_{0}} .
$$

Therefore, the increase in velocity due to flow focusing is expected to be of the order of

$$
\Delta V \sim a \Delta t \sim V_{0} \cos \theta
$$




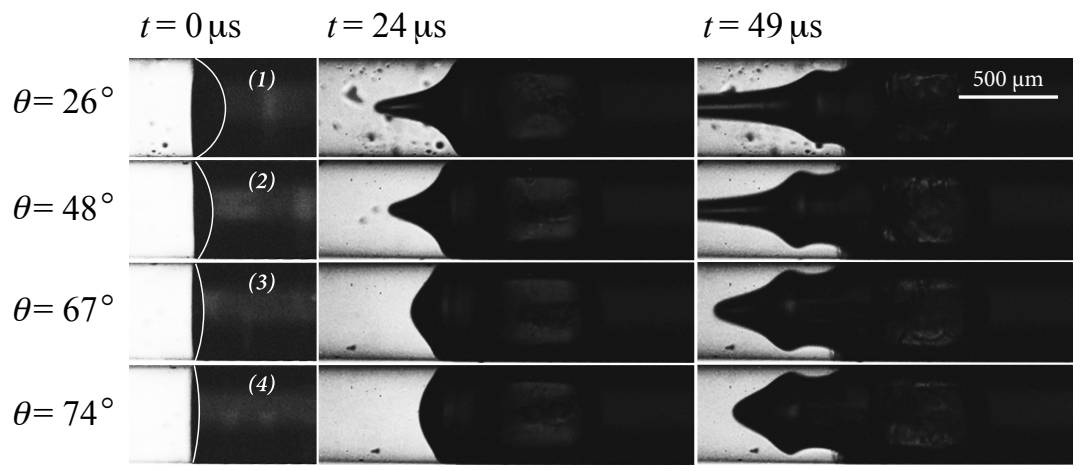

Figure 2.5: Images showing the effect of varying initial contact angle on the jet shape. The contact angles are as follows: (1) is at $26^{\circ},(2)$ is at $48^{\circ},(3)$ is at $67^{\circ}$ and (4) is at $74^{\circ}$. The first image shows the liquid in the capillary prior to laser focusing, illustrating the contact angle and meniscus shape. The second image in each set is taken $24 \mu \mathrm{s}$ after shooting the laser, and the third image follows $25 \mu$ s later. (The movies are available as supplemental material (1) for [figure5-1.mov], (2) figure5-2.mov, (3) figure5-3.mov and (4) figure5-4.mov.)

Thus, the velocity $V$ resulting from the focusing may be expected to be given by

$$
V \sim V_{0}+\Delta V=V_{0}(1+\beta \cos \theta)
$$

where $\beta$ is a constant. With $\beta$ approximately 13 and $V_{0}$ approximately $2.5 \mathrm{~m} / \mathrm{s}$, this relation provides reasonable fits of the data. A more elaborated model will be proposed by Peters et al. [27]. The curve $1-\sin \theta$ suggested by Antkowiak et al. [23] is also shown in figure 2.6. Though the latter model is aimed to describe the initial velocity of the jet, its trend agrees with our experimental data.

\subsubsection{Distance $H$ between laser focus and free surface}

Figure 2.7 shows the experimental results of the asymptotic jet tip velocity $V_{j}$ for the case where the distance $H$ between the laser spot and the free surface is varied. Two different capillary tubes are used with diameters of $200 \mu \mathrm{m}$ and $500 \mu \mathrm{m}$ and two different energy levels $E$. The data shows that, for both tube diameters, $V_{j}$ is inversely proportional to the distance $H$ over a decade. This dependence is particularly well satisfied at the lower energy level $E$. It is well known that the pressure amplitude of a locally generated shock wave in a free fluid decreases inversely with the distance. In our conditions, the fluid is confined within the tube but the size of the initially vaporized liquid is at least one order of magnitude smaller than the tube diameter so 


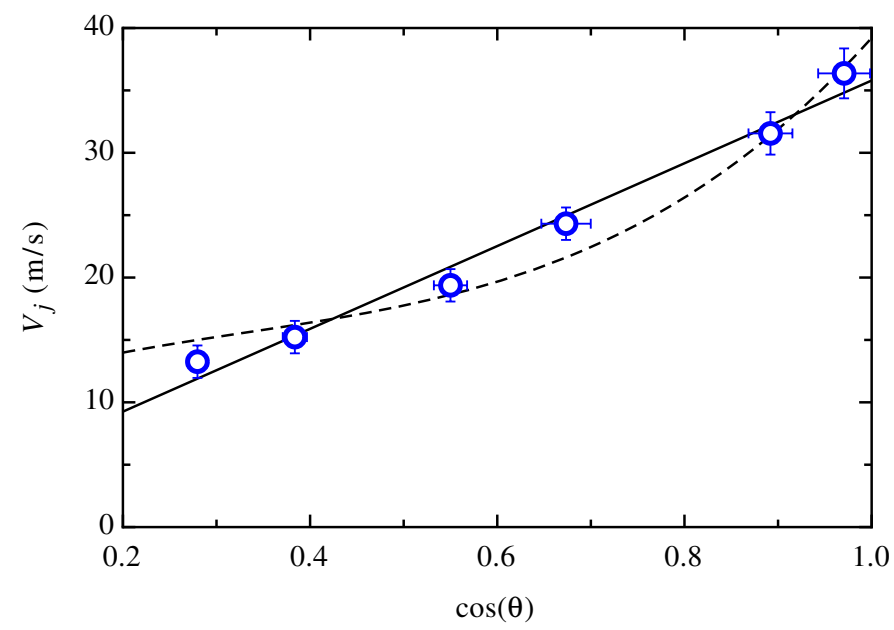

Figure 2.6: Jet velocity $V_{j}$ as a function of the cosine of the contact angle. The solid line is a linear fit to the data. The dashed line is a $1-\sin \theta$ fit to the data suggested by Antkowiak et al. [23].

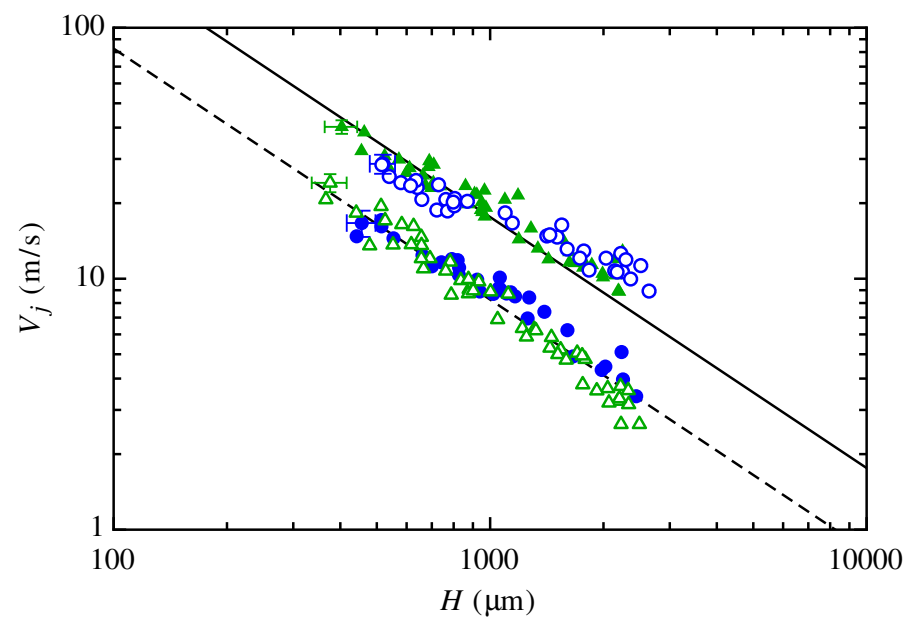

Figure 2.7: Jet velocity $V_{j}$ as a function of the distance $H$ between the laser spot and the free surface for different capillary diameters and energies. The triangles represent the data for the $200 \mu \mathrm{m}$ tube at $E=232 \mu \mathrm{J}(\Delta)$ and $E=165 \mu \mathrm{J}(\triangle)$ and the circles show the results for the $500 \mu \mathrm{m}$ tube at $E=458 \mu \mathrm{J}$ (०) and $E=305 \mu \mathrm{J}$ (०). The solid and dashed lines are showing a -1 power law. Error bars are plotted for the two different capillary diameters, these are similar for the rest of the data. 
that this geometrical attenuation may play a role. Another possibility is that there is viscous attenuation of the shock strength due to the no-slip condition at the tube wall (see e.g. [28, 29]). Therefore, the shock pressure at the meniscus decreases with increasing distance $H$. Since the experiments show that the velocity is inversely proportional to the distance, this suggests that the velocity is proportional to the pressure.

\subsubsection{Absorbed energy $E$}

The next parameter of interest is the energy absorbed by the liquid in the capillary tubes. For this part of the study, the distance between the laser spot and the meniscus is kept constant at $410 \pm 40 \mu \mathrm{m}, 390 \pm 40 \mu \mathrm{m}$ and $600 \pm 40 \mu \mathrm{m}$ for the $50 \mu \mathrm{m}$, $200 \mu \mathrm{m}$, and $500 \mu \mathrm{m}$ diameter tubes, respectively. Figure 2.8 (a) shows $V_{j}$ vs. absorbed energy for different tube diameters. The data are well fitted by a linear function with a positive intercept at $V_{j}=0$. This intercept embodies the existence of a certain threshold $E_{\text {heat }}$ below which no jet is formed. The threshold values for the $50 \mu \mathrm{m}, 200 \mu \mathrm{m}$, and $500 \mu \mathrm{m}$ capillary tubes are approximately $20 \mu \mathrm{J}, 100 \mu \mathrm{J}$, and $200 \mu \mathrm{J}$, respectively. Thus, the threshold value is an increasing function of the tube diameter $D$. It is interesting to note that, for the $50 \mu \mathrm{m}$ capillary tube, the linear relation between $V_{j}$ and $E-E_{\text {heat }}$ is preserved even when the jet speed becomes supersonic as shown in figure 2.8 (b). The absorbed energy for all cases is of the order of $10^{9} \mathrm{~J} / \mathrm{m}^{3}$, which is of the same order as the vaporization enthalpy of water at normal conditions. The experimental results in $\S 2.4 .2$ suggest that the velocity is proportional to the pressure. This linear relation indicates that the pressure of the shock wave at the meniscus is proportional to the absorbed energy.

The slopes of the data for the $50 \mu \mathrm{m}, 200 \mu \mathrm{m}$, and $500 \mu \mathrm{m}$ diameter tubes are $3.09,0.26$ and $0.12 \mathrm{~m} /(\mathrm{s} \cdot \mu \mathrm{J})$, respectively. Thus, for the same absorbed energy, the jet speed decreases with increasing tube diameter. The effects of the diameter $D$ on the jet velocity are discussed further in $\S 2.4 .4$.

In figure 2.8(b), no data points at even higher $V_{j}$ could be acquired because in the current configuration if the energy $E$ becomes too high, the glass tube breaks at the point where the laser focuses.

\subsubsection{Diameter of micro-capillary $D$}

The study of the diameter dependence of the jet velocity encounters the difficulty that the range of absorbed energy for the different tube diameters does not overlap. On the basis of previous results reported above, it is known that, to a good accuracy, $V_{j}$ is proportional to $\left(E-E_{\text {heat }}\right)$ and $(1+\beta \cos \theta)$ and inversely proportional to $H$. We 

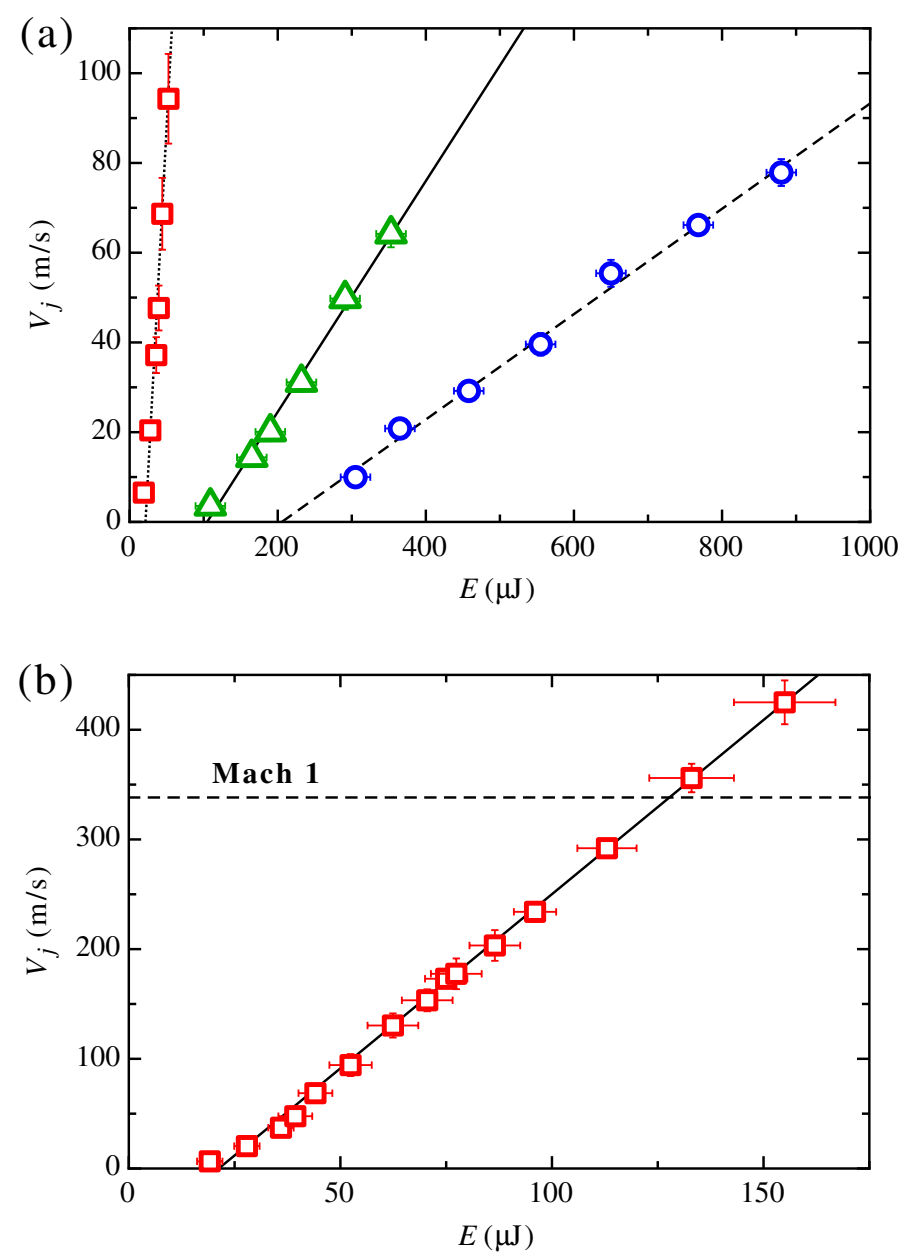

Figure 2.8: (a) Jet velocity $V_{j}$ as a function of the energy absorbed by the liquid in the capillary tubes. The squares $(\square)$, triangles $(\triangle)$ and circles $(\circ)$ represent the data for capillary tubes with $50 \mu \mathrm{m}, 200 \mu \mathrm{m}$, and $500 \mu \mathrm{m}$ inner diameter, respectively. Every data point consists of at least three measurements. The lines are linear fits to the data. (b) Jet velocities $V_{j}$ of supersonic microjets as a function of the energy absorbed by the liquid in the capillary tubes with $50 \mu \mathrm{m}$ inner diameter. Every data point consists of at least five measurements. The line is a linear fit to the data. Even for the supersonic speeds we see that the linear dependence holds. 


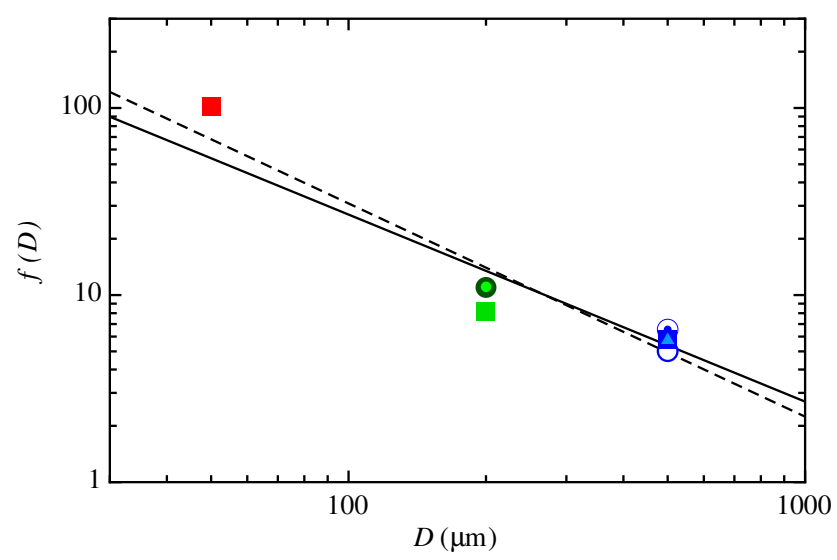

Figure 2.9: Pre-factor $f(D)$ from equation (2.6) versus the capillary diameter. The circles, squares, and diamonds are constants obtained from the energy dependence, distance dependence, and contact angled dependence experiments, respectively. A best fit power law (dashed line) with power -1.14 is shown as well as a line with power -1 as shown in equation (2.7) (solid line), which shows less agreement with the data, in particular for small $D$.

express this dependency by writing

$$
V_{j} \simeq f(D) \frac{\left(E-E_{\text {heat }}\right)(1+\beta \cos \theta)}{H},
$$

where the pre-factor $f(D)$ embodies the dependence on the diameter. By trying power law dependence $f(D)=f_{0} D^{-\alpha}$, the data is best fitted by $\alpha \simeq-1.14$, which may be considered close to -1 .

Especially the data of the $200 \mu \mathrm{m}$ and $500 \mu \mathrm{m}$ tubes agrees well with the line of slope -1 , while that of $50 \mu \mathrm{m}$ is higher than the line with slope -1 . This could be attributed to the fact that different effects, such as laser focusing, start to play an important role at smaller tube sizes due to higher curvature of the tube. According to the impulse pressure description suggested by Antkowiak et al. [23] and the dimensional consideration performed in $\S 2.4$.1, the diameter of the tube should not play a role. A possible reason for this dependence is that the same energy delivered towards the smaller diameter tube will give rise to the faster jet due to the smaller inertia. To strengthen this hypothesis it may be noted that, if the kinetic energy is constant for a given absorbed energy $E$, since the mass of the accelerated liquid is approximately proportional to $D^{2}$, it follows that the velocity would decrease proportionally to $D^{-1}$. From the results that were presented in the previous sections, the following empirical 
law is derived:

$$
V_{j} \simeq C_{0} \frac{\left(E-E_{\text {heat }}\right)(1+\beta \cos \theta)}{H D} .
$$

Calculating $C_{0}$ by fitting the data in figure 2.9 we get $C_{0}=0.0027(\mathrm{~Pa} \cdot \mathrm{s})^{-1}$. It is interesting to note that $C_{0}$ has the dimensions of an inverse dynamic viscosity.

\subsubsection{Focus offset $l_{v}$ and $l_{h}$}

The sensitivity of the jet to the laser focus position relatively to the capillary axis was studied by displacing it in the horizontal $\left(l_{h}\right)$ and vertical directions $\left(l_{v}\right)$ (see figure 2.1(b)). The results are shown in figure 2.10.

Figure 2.10(a) indicates that the jet velocity decreases monotonically for increasing $l_{h}$ all other conditions being held constant. We hypothesize that this trend is explained by a reduced volume of the vaporized liquid. To test this hypothesis, the jet velocity is compared to the initial laser-induced vaporized volume as estimated with the geometrical optics approximation described in Appendix A. As shown in figure 2.10(a), the jet velocity roughly scales with the calculated volume of the liquid vaporized by the laser pulse.

Figure 2.10(b) shows the jet speed versus the vertical displacement $l_{v}$. The jet velocity increases when increasing $l_{v}$ up to $l_{v} \approx 1.5 \mathrm{~mm}$, and then decreases for larger $l_{v}$. The rise is likely caused by the larger area of the capillary tube surface illuminated by the laser, which increases as the laser is moved towards the tube. As the vaporization always occurs at the capillary wall, a larger vapour mass will be created by increasing $l_{v}$. However, when moving the capillary even closer to the objective, the energy per unit area of the laser beam decreases and eventually drops below the threshold required for bubble formation. This situation was also modeled by geometrical optics. As shown in figure 2.10(b), the maximum jet speed and maximum bubble size are found at the same offset. Even though the overall trend is the same, the normalised values of the vaporized volume deviate from those of the jet velocity. This difference is not surprising since there is no reason to think that vaporized volume and jet growth are linearly related to each other. The analysis of these aspects is beyond the scope of this work; they will be studied in the future investigations.

The robustness of this velocity increase is shown in figure 2.11 , where the jet speed versus the vertical focus offset $l_{v}$ is plotted for different diameters down to $50 \mu \mathrm{m}$. Jets with a velocity up to $850 \mathrm{~m} / \mathrm{s}$ could consistently be produced with the smallest capillaries. Measurements beyond this velocity could not be obtained, as the capillary breaks at the position of the laser focus due to the violence of the bubble expansion. We also observed that, at the higher energies, the capillary tip is sheared off by the wall shear stresses exerted by the liquid pushed out of the capillary by the 


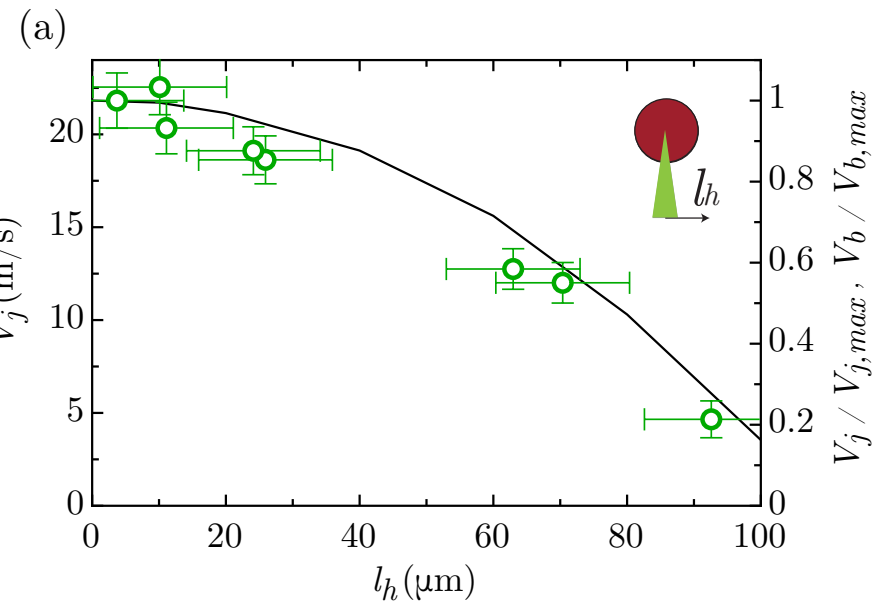

(b)

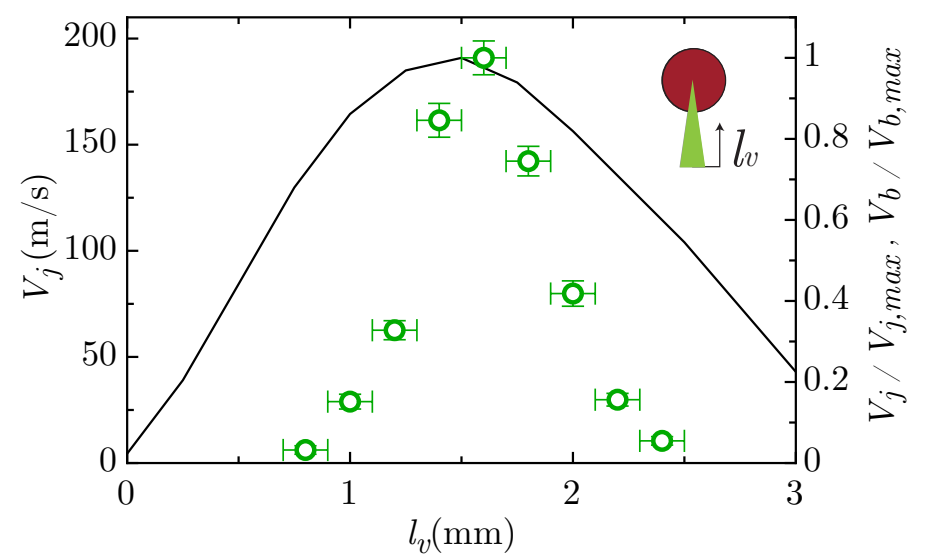

Figure 2.10: (a) Jet velocity $V_{j}(\circ)$ as a function of the horizontal focus displacement of the laser, for the $200 \mu \mathrm{m}$ tube. The black line shows the scaled initial bubble size determined by geometrical optical approximation in both graphs (right axis). (b) Jet velocity $V_{j}$ as a function of the vertical focus displacement of the laser. The inserts show the capillary and the laser focus (triangle) and the directions of $l_{h}$ and $l_{v}$ as seen from the capillary exit. $E$ and $H$ are kept constant per data set. 


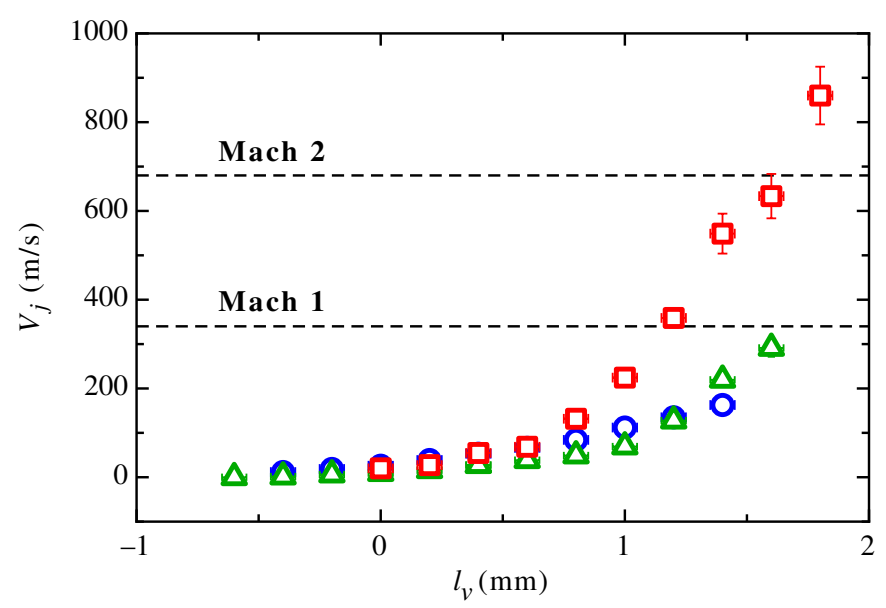

Figure 2.11: Jet velocity $V_{j}$ as a function of the focus offset of the laser, for different capillary diameters. $E$ and $H$ are kept constant per data set. The circles (o) represent measurements for the $500 \mu \mathrm{m}$ tube, the triangles $(\triangle)$ are data for the $200 \mu \mathrm{m}$ tube and the squares $(\square)$ are data for the $50 \mu \mathrm{m}$. Each data point consists of at least three measurements. The input energy is kept constant for each diameter.

expanding bubble following the thin jet formation. It is possible that, with different materials, the velocity of the jet can be increased. When the jet velocity reaches the speed comparable to the speed of sound in water, some limitations to further increases may arise due to the dominance of compressibility effects.

\subsection{Summary and conclusions}

The dynamics of the high-speed microjet generated by laser-induced rapid vaporization of water in a micro-tube has been studied. It has been shown that the jets so generated are able to reach speeds as high as $850 \mathrm{~m} / \mathrm{s}$ with good controllability.

The dependence of the jet velocity upon various controlling parameters has been investigated in a series of experiments the results of which have been summarised in the empirical relationship provided in equation (2.7). This equation shows the effect of the distance between the laser focus and the liquid meniscus at the mouth of the micro-tube, the absorbed laser energy, the liquid-tube contact angle and the tube diameter.

The jet velocity exhibits an inverse proportionality to the tube diameter and to the distance of the laser focus from the free surface, while it is proportional to the 
absorbed energy above a threshold value. The velocity is very strongly dependent on the curvature of the interface, which is a function of the tube-liquid contact angle. This result suggests that the mechanism underlying the phenomenon is the focusing of the shock wave produced by the nanosecond-scale phase change induced by the absorption of the laser energy similarly to the phenomenon exploited in shaped charges [18]. Thus, the jet velocity is critically dependent on the amount of liquid vaporized and on its distance from the free surface, both of which can be varied by varying the position of the laser focus.

In particular, it has been found that the effect of the offset of the laser focus with respect to the tube axis has a strong and very non-trivial effect. To elucidate the origin of this result we have used a geomterical-optics construction coupled with the Beer-Lambert law to determine approximately the size of the region where vaporization occurs and the absorbed energy. While still preliminary, the results of this analysis are in general agreement with the data. Further theoretical and numerical investigations of the jetting phenomenon will be addressed in Peters et al. [27]. The insights gained through this research and the ability to generate focused, controllable, and high velocity microjets opens new doors for the realization of reliable needle-free drug delivery systems.

\section{Appendix: Estimate of the liquid energy absorption by geo- metrical optics}

A geometrical optics approximation was developed to analyze energy absorption of the laser beam energy absorption by the liquid. The three main components of this calculation are: (1) splitting up the Gaussian laser beam into many different rays each one with a representative energy; (2) following the path of each ray through the capillary, including (full or partial) wall-reflections; (3) using the Beer-Lambert law to model the local energy absorbed by the liquid. This law describes the local irradiance $I$, and is given by $I=I_{0} \cdot 10^{-\varepsilon s}$, with $I_{0}$ the beam irradiance at the cylinder outer surface of the tube, $\varepsilon$ the absorption coefficient, $s$ the arc length along the partial of the ray propagating in the liquid. The absorption coefficient was measured as $\varepsilon=84 \cdot 10^{3} \mathrm{~m}^{-1}$; the local energy loss of a ray equals $\partial I / \partial s$.

The liquid volumes are discretized into cells constituting a grid around the central loss area. The local energy lost by each ray in each cell was calculated from the Beer-Lambert law and add to the liquid in the cell. Subsequently, for each cell, the total energy absorbed over the duration of the light pulse was compared with an estimate of the energy necessary for evaporation at room conditions, namely $E_{b o i l}=$ $\rho\left(C_{p} \Delta T+\Delta H_{\text {vap }}\right) \Delta V_{c}$, with $\rho$ the liquid density, $\Delta T=80 \mathrm{~K}$ the difference between 
the initial liquid temperature and the boiling temperature, $C_{p}=4181 \mathrm{~J} /(\mathrm{kgK})$ the heat capacity at constant pressure, $\Delta H_{v a p}=2.26 \mathrm{MJ} / \mathrm{kg}$ the vaporization enthalpy, and $\Delta V_{c}$ the cell volume. If the energy absorbed by a cell exceeded $E_{b o i l}$, the cell volume was assumed to be vaporized instantaneously. Summation of the vaporized-cell volumes then provided a measure for the initial bubble volume and position.

\section{References}

[1] J. Eggers and E. Villermaux, Reports on Progress in Physics 71, 036601 (2008).

[2] J. de Jong, R. Jeurissen, H. Borel, M. van den Berg, H. Wijshoff, H. Reinten, M. Versluis, A. Prosperetti, and D. Lohse, Physics of Fluids 18, 121511 (2006).

[3] H. Wijshoff, Physics Reports 491, 77 (2010).

[4] W. van Hoeve, S. Gekle, J. H. Snoeijer, M. Versluis, M. P. Brenner, and D. Lohse, Physics of Fluids 22, 122003 (2010).

[5] C. Ohl, M. Arora, R. Dijkink, V. Janve, and D. Lohse, Applied Physics Letters 89, 074102 (2006).

[6] R. Dijkink and C. Ohl, Applied Physics Letters 93, 254107 (2008).

[7] A. Zijlstra, T. Janssens, K. Wostyn, M. Versluis, P. M. Mertens, and D. Lohse, Solid State Phenomena 145-146, 7 (2009).

[8] J. Blake, Y. Tomita, and R. Tong, Applied Scientific Research 58, 77 (1997).

[9] R. Bergmann, D. van der Meer, M. Stijnman, M. Sandtke, A. Prosperetti, and D. Lohse, Phys. Rev. Lett. 96, 154505 (2006).

[10] R. Bergmann, D. van der Meer, S. Gekle, A. van der Bos, and D. Lohse, Journal of Fluid Mechanics 633, 381 (2009).

[11] S. T. Thoroddsen, T. G. Etoh, and K. Takehara, Physics of Fluids 19, 042101 (2007).

[12] S. Gekle, A. van der Bos, R. Bergmann, D. van der Meer, and D. Lohse, Phys. Rev. Lett. 100, 84502 (2008).

[13] S. Gekle and J. M. Gordillo, Journal of Fluid Mechanics 663, 293 (2010).

[14] S. Mitragotri, Nature Reviews Drug Discovery 5, 543 (2006).

[15] J. Schramm-Baxter and S. Mitragotri, Journal of Controlled Release 97, 527 (2004).

[16] HAN, T. \& YOH, J. J. 2010 A laser based reusable microjet injector for transdermal drug delivery. Journal of Applied Physics 107 (10), 103110.

[17] Menezes, V., Kumar, S. \& Takayama, K. 2009 Shock wave driven liquid microjets for drug delivery. Journal of Applied Physics 106 (8), 086102.

[18] G. Birkhoff, D. P. MacDougall, E. M. Pugh, and G. Taylor, Journal of Applied Physics 19, 563 (1948).

[19] J. P. Curtis and R. J. Kelly, Journal of Applied Physics 75, 7700 (1994).

[20] J. Petit, V. Jeanclaude, and C. Fressengeas, Journal of Applied Physics 98, 123521 (2005).

[21] É. Lorenceau, D. Quéré, J. Ollitrault, and C. Clanet, Physics of Fluids 14, 1985 (2002).

[22] R. Bergmann, E. de Jong, J.-B. Choimet, D. van der Meer, and D. Lohse, Journal of Fluid Mechanics 600, 19 (2008).

[23] A. Antkowiak, N. Bremond, S. Le Dizès, and E. Villermaux, Journal of Fluid Mechanics 577, 241 (2007). 
[24] C. Sun, E. Can, R. Dijkink, D. Lohse, and A. Prosperetti, Journal of Fluid Mechanics 632, 5 (2009).

[25] A. L. Sumner, E. J. Menke, Y. Dubowski, J. T. Newberg, R. M. Penner, J. C. Hemminger, L. M. Wingen, T. Brauers, and B. J. Finlayson-Pitts, Physical Chemistry Chemical Physics 6, 604 (2004).

[26] A. van der Bos, A. Zijlstra, E. Gelderblom, and M. Versluis, Experiments in Fluids 51, 1 (2011).

[27] I. R. Peters, Y. Tagawa, N. Oudalov, C. Sun, D. van der Meer, A. Prosperetti, and D. Lohse, Journal of Fluid Mechanics 719, (2011).

[28] G. Mirshekari and M. Brouillette, Shock Waves 19, 25 (2009).

[29] D. Ngomo, A. Chaudhuri, A. Chinnayya, and A. Hadjadj, Computers \& Fluids 39, 1711 (2010). 


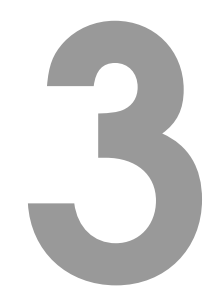

\section{Microdroplet impact at very high velocity ${ }^{*} \dagger$}

Water microdroplet impact at velocities up to $100 \mathrm{~m} / \mathrm{s}$ for droplet diameters from 12 to 100 $\mu \mathrm{m}$ is studied. This parameter range covers the transition from capillary-limited to viscositylimited spreading of the impacting droplet. Splashing is absent for all measurements; the droplets always gently spread over the surface. The maximum spreading radius is compared to several existing models. The model by Pasandideh-Fard et al. [1] agrees well with the measured data, indicating the importance of a thin boundary layer just above the surface, in which most of the viscous dissipation in the spreading droplet takes place. As explained by the initial air layer under the impacting droplet, a contact angle of 180 degrees is used as model input.

${ }^{*}$ Published as: C.W. Visser, Y. Tagawa, C. Sun, D. Lohse, Microdroplet impact at very high velocity. Soft Matter 8 (41), 10732-10737 (2012)

${ }^{\dagger}$ Experiments, data processing, and first draft by CWV. 


\subsection{Introduction}

High-pressure spray cleaning, droplet-wall interactions in diesel engines, and plasma spraying are notable examples of processes in which high-speed impact of small droplets on a solid surface is a key phenomenon. In these applications, droplets with a characteristic size of 1 to $100 \mu \mathrm{m}$ and a velocity of order $100 \mathrm{~m} / \mathrm{s}$ impact on a solid surface [2-4]. Despite this industrial interest, microscale droplet impact at very high velocity $\left(U_{0}>50 \mathrm{~m} / \mathrm{s}\right)$ has only been studied for solidifying metal droplets [5]. This is mainly due to the challenging parameter regime: very high spatial and temporal resolutions are required to study the relevant phenomena. In addition, it is difficult to create impact events at these velocities. An understanding of the phenomena of high-speed micro-sized droplet impact is thus lacking $[6,7]$.

In this work, we aim to extend current results in three ways. First, we present a novel high-velocity droplet generation, by using a method to create ultrafast liquid jets. Second, using high-speed imaging, the impact dynamics is studied. Third, a quantitative investigation of the maximum spreading radius will be presented and compared to existing models, to improve our understanding of droplet spreading.

\subsection{Parameter space}

To compare our results to previous work, a phase diagram of the droplet size $\left(D_{0}\right)$ and impact speed $\left(U_{0}\right)$ is plotted in figure 3.1 (a). Most work up to now has focused on impact dynamics of droplets with a size of $\sim 1 \mathrm{~mm}$ in diameter. The studies on microdroplet impact were mainly at relatively low speed (up to $10 \mathrm{~m} / \mathrm{s}$ ) [5, 8, 9]. Our study connects these previous investigations, in particular those of water microdroplets at lower velocies [8] and metal microdroplet impact at very high velocities [5].

Figure 3.1 (b) shows a phase diagram of the achieved Reynolds and Weber numbers of experimental droplet studies. The Weber number is defined as We $=\rho D_{0} U_{0}^{2} / \sigma$, where $\sigma$ the surface tension and $\rho$ the density. The Reynolds number is given by $\operatorname{Re}=\rho D_{0} U_{0} / \mu$ where $\mu$ represents dynamic viscosity. As shown in figure 3.1 (b), the Re-We values of our measurements largely overlap with previous data. However, our data have been taken for microdroplets instead of mm-sized droplets as indicated in figure 3.1 (a). As the droplet size is a key control parameter for impact dynamics, various models developed to describe the mm-sized droplet impact may not hold for microdroplet impact dynamics. The large impact velocity also explains why our data have relatively high Weber numbers for given Reynolds numbers.

Our data cover the transition between the capillary- to the viscosity-dominated 
limits of droplet spreading [10], as shown by the solid line in figure 3.1 (a) (an explanation of this transition is provided below). So far, to investigate this transition, liquids of different viscosities and surface tension were required in order to achieve a sufficient coverage of the Re-We parameter space. Using droplets of microscopic scales lowers the Weber number for which this transition takes place, allowing to study the transition region with a single liquid.

\subsection{Experimental setup}

To create high-velocity microdroplets $\left(U_{0} \geq 10 \mathrm{~m} / \mathrm{s}\right)$, we make use of a new method to create ultrafast liquid jets [18], as sketched in figure 3.2 (a). In a nutshell, the method works as follows. By focusing a laser pulse with a microscope objective, a vapor bubble is created in a capillary tube, by laser-induced cavitation. From this bubble, a shock wave travels to the meniscus, which in turn forms a liquid jet thanks to flow focusing. Subsequently, this jet breaks up into tiny droplets with a velocity similar to the jet velocity (figure $3.2(\mathrm{~b})$ ).

It is found that the tube diameter is a key control parameter for both the jet diameter and the jet tip velocity [18]. Therefore, capillaries with different diameters are used to create a range of droplet sizes and velocities. In addition, the laser energy and the distance between the laser focus and the meniscus are varied to generate droplets at different velocities for a given tube diameter [18], resulting in the diameter- and velocity ranges, as shown in figure 3.2 (c). With this method, approximately 170 droplet impacts on a dry surface were examined. For the sake of clarity, the data is binned in the figure.

To create droplets at velocities between 1 and $10 \mathrm{~m} / \mathrm{s}$, a commercially available Microdrop dispenser (MD-140-752, Microdrop technologies, Germany) is used. By varying the input voltage between $60 \mathrm{~V}$ and $160 \mathrm{~V}$ per pulse, a range of velocities is covered. The droplet diameter ranges from $40 \mu \mathrm{m}$ to $80 \mu \mathrm{m}$, as shown in figure 3.2 (c).

A standard microscope slide was used as an impact plate, which is placed above the tip of the capillary tube. Atomic force microscope measurements indicated a roughness $R_{a}$ below $10 \mathrm{~nm}$. The droplet impact is visualized from the side. After each impact measurement, the glass plate was cleaned with ethanol and dried. Frequent checks with a $10 \times$ optical microscope (after the cleaning procedure) indicated that this method usually resulted in an optically clean surface, i.e., hardly any paper fibers or dirt were sticking to the surface. To increase the absorption rate from the green laser, the liquid used work was a standard water-based blue inkjet printer ink, with density $\rho=998 \mathrm{~kg} / \mathrm{m}^{3}$, surface tension $\sigma=72 \mathrm{mN} / \mathrm{m}^{2}$, and viscosity $\mu=10^{-3} \mathrm{~Pa} \cdot \mathrm{s}$. 

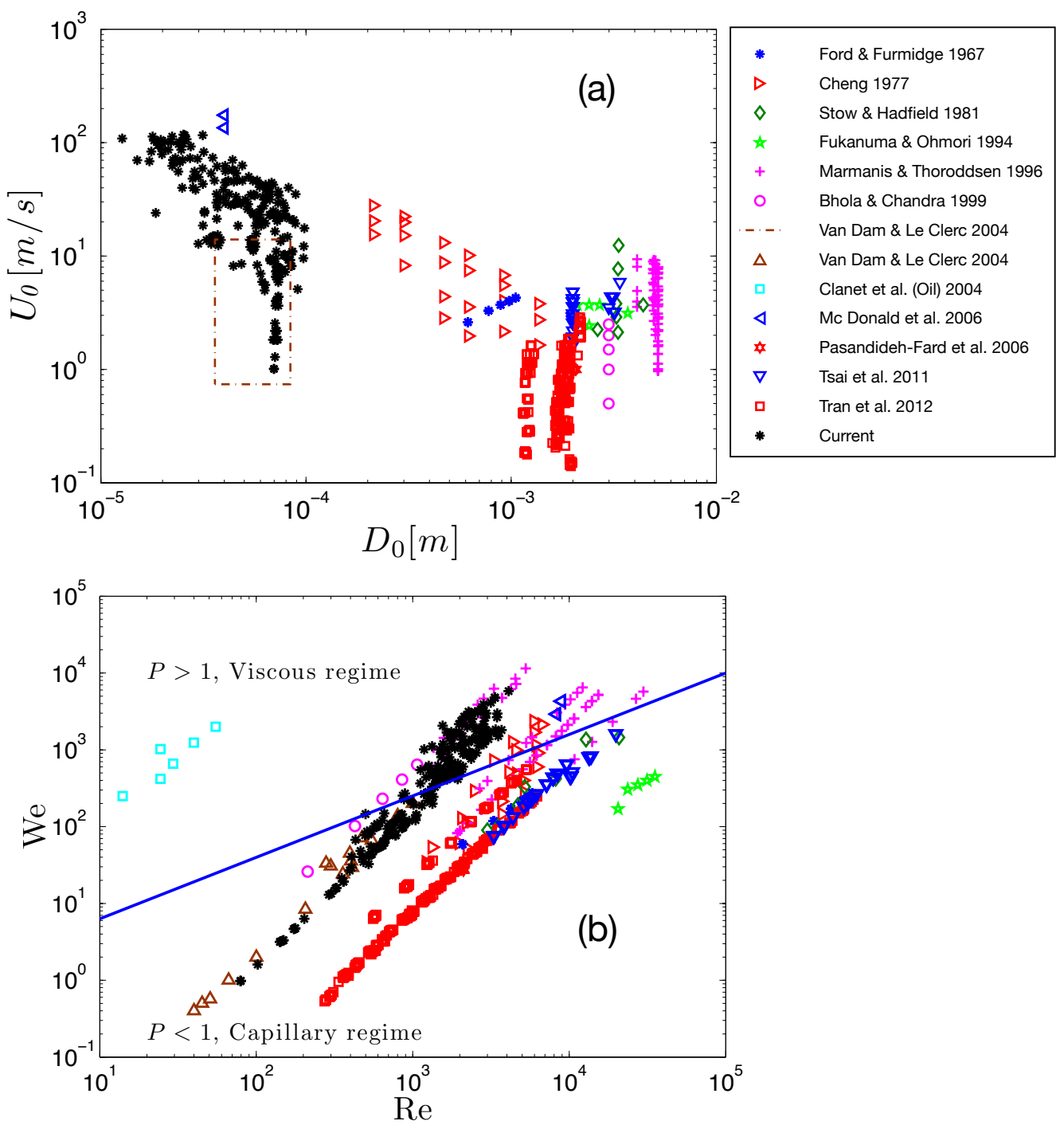

Figure 3.1: Parameter space of (a) droplet velocities and radii and (b) Weber and Reynolds numbers for various droplet impact experiments [1, 5, 8-17]. The solid blue line signals $P$ $=1\left(P\right.$ is defined as $P=\mathrm{We} / \mathrm{Re}^{4 / 5}$ by Clanet et al. $\left.[10]\right)$ and separates the capillary regime with $P<1$ from the viscous regime with $P>1$. 
(a)

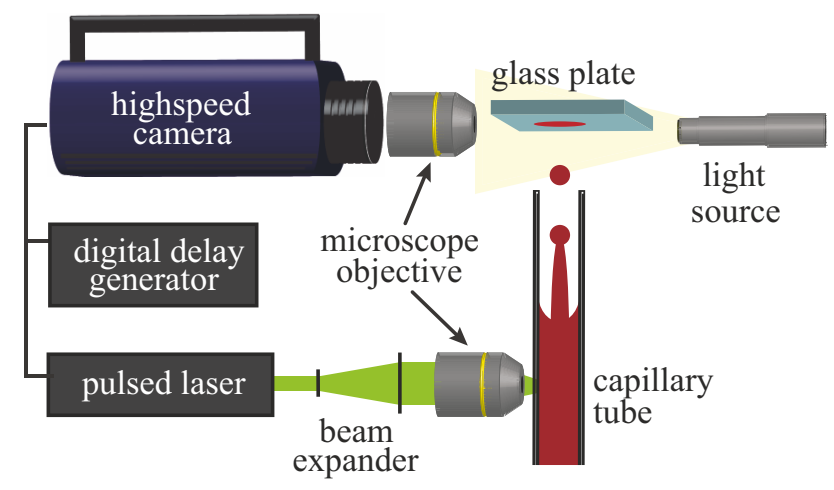

(b)

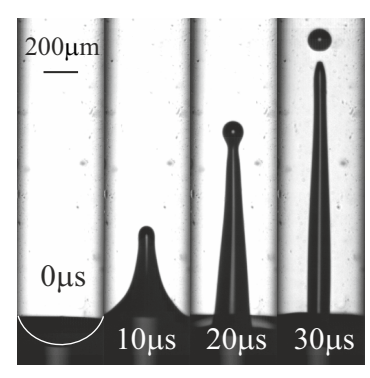

(c)

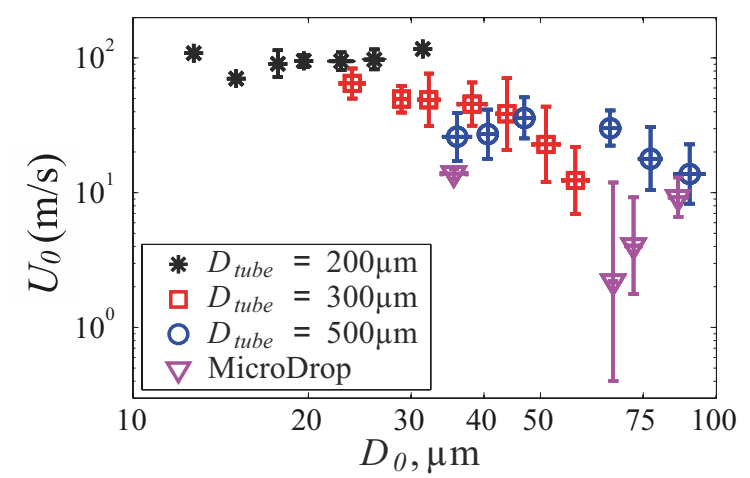

Figure 3.2: (a) Setup used for the generation of fast droplets. (b) Jet generation and breakup at different instants. (c) Range of the achieved droplet velocities and radii, for different droplet generator settings. Capillary tube diameters are decreased from $500 \mu \mathrm{m}$ to $200 \mu \mathrm{m}$ to generate smaller and faster droplets. In addition, to create slow droplets, a MicroDrop apparatus is used. As approximately 230 measurements were performed, the data are binned. The bars represent one standard deviation.

Magnifications of $10 \times$ to $40 \times$ are obtained by combining a standard Olympus $10 \times$ objective with an adjustable $12 \times$ zoom lens (Navitar 1-50015). An Olympus ILP-1 light source is used for illumination. An ultra high-speed camera (Shimadzu HPV-1) is used to study the microdroplet impact dynamics, at recording rates of $1.25 \cdot 10^{5}$ to $10^{6}$ frames per second. For the fastest droplets $\left(U_{0} \approx 100 \mathrm{~m} / \mathrm{s}\right)$, the impact duration is approximately $\tau=D_{0} / U_{0} \approx 0.2 \mu \mathrm{s}$, which is below the temporal resolution of the camera. Thus, completely capturing of such events requires even higher frame rates [19] or pulsed illumination, e.g. as used in Ref. [20]. However, 
these techniques require a level of control of the moment of impact which is not achieved with the present setup. The high-speed camera has a minimum shutter time of $500 \mathrm{~ns}$. Therefore, at high impact velocities, substantial motion blur is observed (e.g. figure 3.3 (c)), as the shutter time approaches $\tau$. Still, with the current setup, the presence of splashing could be assessed and the maximum spreading radius could be observed in an entirely new parameter regime.

Special care was taken to ensure that the data analysis was reliable and consistent. First, the raw Shimadzu movie files were read into Matlab. The relevant frames of each movie were selected manually, as well as the area of interest, resulting in image sequences as shown in figure 3.3. After contrast-enhancement, the experiments were inspected manually and removed if the images were unclear. Then, in the first two frames of interest, the droplet was identified manually. Subsequently, a crosscorrelation method between sub-areas of the frames (these sub-areas, with a width of 3 times the droplet size and a height dependent on the velocity estimated, contain the droplet image) was used to determine the droplet position in each frame. From the correlation, the velocity was calculated. To determine the droplet size before and after spreading, the images were converted to black-and-white. Using standard Matlab methods, coherent structures were automatically labelled. Using thresholds for eccentricity and size, the droplet area was identified from the labeled areas. The width of the labeled area (a standard property in Matlab) then provided the droplet size. As a check, a diameter-sized bar was plotted over the centre of the droplet. The result was adjusted manually if necessary, to match the maximum droplet radius. For graphs Figs. 3.2c and 3.4, the data was binned. The error bars represent one standard deviation. Each data point in Fig. 3.4 (to be discussed below) contains at least 4 measurements.

\subsection{Results}

\subsubsection{Impact Phenomenology}

In general, the droplet impact process can be divided into the following phases. When the droplet approaches the solid surface, the air between the falling drop and the surface is strongly squeezed, leading to a pressure buildup in the air under the drop. The enhanced pressure results in a dimple formation in the droplet and an air layer development between the droplet and the target plate [21-23]. Before the droplet wets the surface, the liquid moves on top of this air cushion. The droplet extends in the radial direction until it reaches a maximum spreading radius. In this phase, splashing can occur $[7,16,21,24-26]$. Finally, the droplet completely wets the 

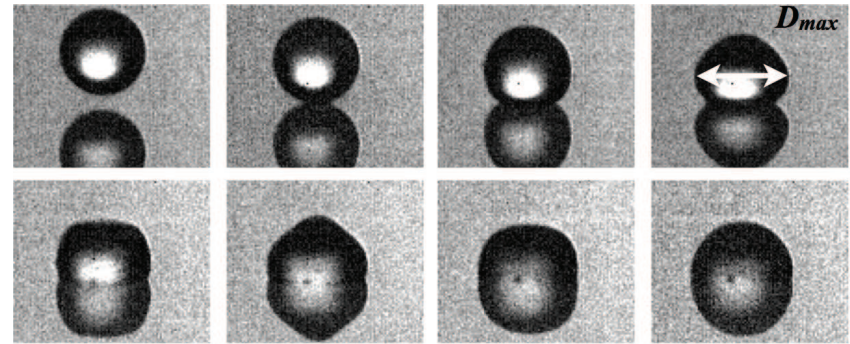

(a)
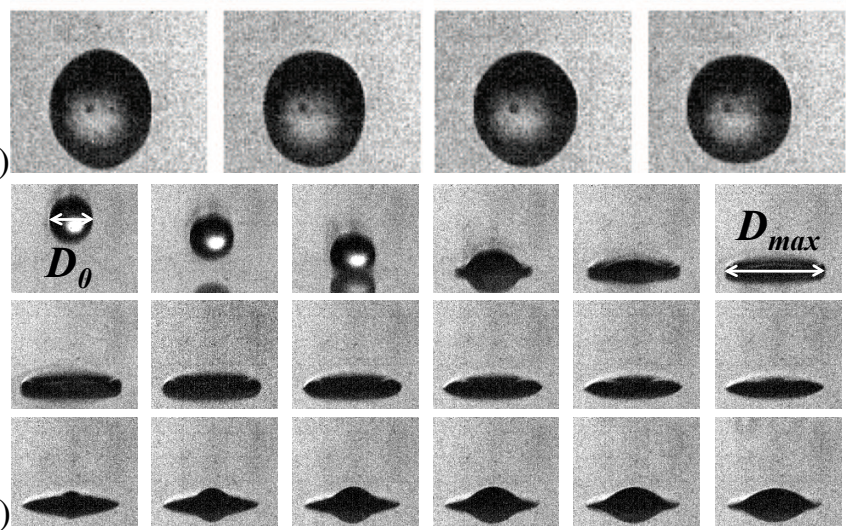

(b)

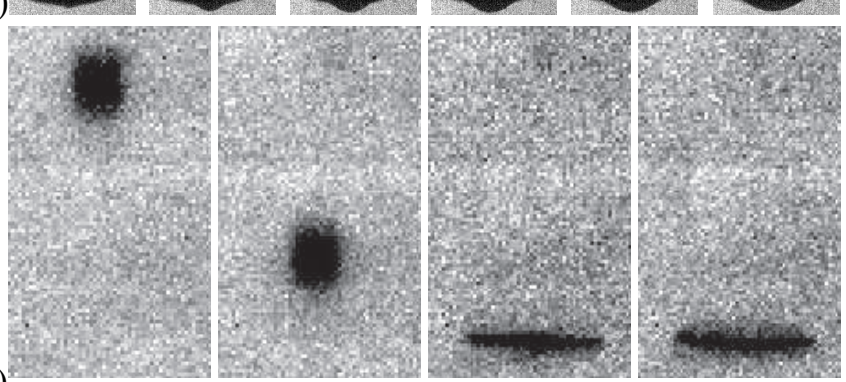

(c)

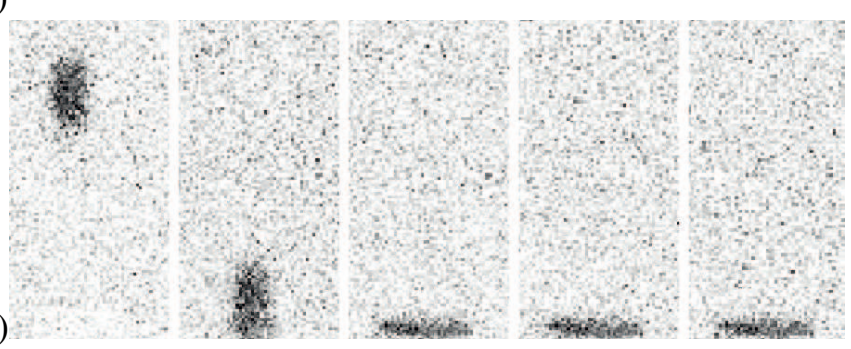

Figure 3.3: Time series of droplet impact at different impact velocities. (a) $U_{0}=0.7 \mathrm{~m} / \mathrm{s}$, $D_{0}=70 \mu \mathrm{m}, \mathrm{We}=0.52, \mathrm{Re}=56$, and the time lag $\delta t$ between the frames is $8 \mu \mathrm{s}$. Droplet oscillations and air bubble entrapment can clearly be seen. (b) $U_{0}=7.7 \mathrm{~m} / \mathrm{s}, D_{0}=71 \mu \mathrm{m}$, $\mathrm{We}=60, \mathrm{Re}=613$, and $\delta t=4 \mu \mathrm{s}$. Spreading into a thin center sheet and a thicker rim are observed, followed by oscillations and partial withdrawal of fluid from the rim towards the droplet center. (c) $U_{0}=73 \mathrm{~m} / \mathrm{s}, D_{0}=23 \mu \mathrm{m}$, We $=1.8 \cdot 10^{3}$, Re $=1.9 \cdot 10^{3}$, and $\delta t=1 \mu \mathrm{s}$. The details of the spreading phase can no longer be resolved. (d) $U_{0}=100 \mathrm{~m} / \mathrm{s}, D_{0}=20 \mu \mathrm{m}$, $\mathrm{We}=3 \cdot 10^{3}, \operatorname{Re}=2.3 \cdot 10^{3}$, and $\delta t=1 \mu \mathrm{s}$. 
surface and an air bubble is entrapped [23].

The first aim of this work is to assess the dynamics of microdroplet impact, i.e. to find out whether the drop is in the splashing or gentle spreading regime. The latter one is defined by droplet deformation into a pancake shape, without satellite droplet formation. Time series of droplet impacts and the subsequent spreading phases are shown in figure 3.3. For these figures, the velocity ranges from $0.7 \mathrm{~m} / \mathrm{s}$ to $100 \mathrm{~m} / \mathrm{s}$.

At low velocity $\left(U_{0}=0.7 \mathrm{~m} / \mathrm{s}\right)$, initial flattening of the droplet bottom is observed, as shown in figure 3.3 (a). Subsequently, the droplet spreads over the substrate into a (virtually) half-dome shaped cap. During spreading, the droplet starts to oscillate and comes to rest only after the droplet has reached its maximum diameter. Additionally, air bubble entrainment is observed (the small black dot, just left of the droplet center). These phenomena are consistent with what had been reported in Ref. [8], which includes a detailed discussion on the droplet oscillation frequency and the size and cause of the bubble/cushioning entrained [16, 22, 23, 27]. At medium velocities (7.7 $\mathrm{m} / \mathrm{s}$, figure 3.3 (b)), the droplet deforms into a disc-like structure. Here, the central impact area is a sheet-like structure surrounded by a thicker rim. Again, capillary oscillations were observed. Finally, even at very high velocities (figures 3.3 (c) and 3.3 (d)), still no splashing is observed. Thus, we conclude that gentle impact occurs for all velocities and droplet sizes investigated in the present work. Apart from the surrounding pressure and the surface roughness, the splashing dynamics of course also depends on the droplet size and for these small droplets the surface tension is strong enough to prevent splashing.

\subsubsection{Maximum spreading}

Now the maximum spreading radius will be determined and compared against various models. As knowledge of the maximum spreading diameter is of paramount importance for industrial applications, a plethora of models has been developed [1, 9, $10,28-32]$. It is generally agreed on that the spreading is limited by either viscosity or surface tension. Therefore, a key issue is to define which of these is dominant. To study this issue, we will first briefly summarize several models.

Assuming an inviscid liquid, the maximum spreading is limited by the surface tension. Balancing the Laplace pressure force with the inertial deceleration of the drop, a scaling of $D_{\max } / D_{0} \sim \mathrm{We}^{1 / 4}$ is obtained $[9,10]$. This scaling is remarkably robust $[10,14,16]$ in the capillary regime. As shown in figure 3.4 (a), the data of mm-sized droplets impact on superhydrophobic surfaces obtained by Tsai et al. [16] well agree with this $1 / 4$ scaling law.

Another limiting case is to completely ignore surface tension and assume that the maximum spreading radius is limited by the viscous dissipation during droplet 
spreading [10]. This yields $D_{\max } / D_{0} \sim \mathrm{Re}^{1 / 5}$ as scaling law, which holds well for $\mathrm{mm}$-sized droplets in the viscous regime as shown in Ref. [10].

To quantify the transition between the viscous and the capillary regimes, Clanet et al. [10] defined the parameter $P=\mathrm{We} / \operatorname{Re}^{4 / 5}$. For $P<1$, a surface-tension dominated regime is expected (i.e. $D_{\max } / D_{0} \sim \mathrm{We}^{1 / 4}$ ), whereas a viscous scaling is predicted for $P>1$, yielding the previously mentioned $D_{\max } / D_{0} \sim \operatorname{Re}^{1 / 5}$. In figure 3.4 (a), the transitional Weber number $W e_{t}$, defined by $P=1$, for droplets of $D_{0}=2 \mathrm{~mm}$ and $50 \mu \mathrm{m}$ are plotted. For $2 \mathrm{~mm}$-sized droplets, $\mathrm{We}_{\mathrm{t}} \simeq 3 \times 10^{3}$, whereas it decreases to $\mathrm{We}_{\mathrm{t}} \simeq 2 \times 10^{2}$ for $50 \mu \mathrm{m}$-sized ones. As shown in figure 3.4 (a), the present microdroplet data are in between the transition regime from the capillary regime to the viscous regime. For a given We, the microdroplet spreading is lower than that of mm-sized droplets due to the viscous effects. This combination of two simple models provides a decent first description of the impact dynamics. However, more detailed models are possible and have been developed. As described by Chandra \& Avedisian [29], the dissipated energy equals the work done, and can thus be estimated by

$$
W=\int_{0}^{\tau} \int_{V_{v}} \phi \approx V_{v} \tau \phi \approx V_{v} \tau \mu\left(\frac{U_{0}}{L}\right)^{2}
$$

where $\phi$ the dissipation function, estimated as $\mu\left(U_{0} / L\right)^{2}[10,29]$, with $\tau$ the typical impact timescale, $V_{v}=\pi L D_{\max }^{2}$ the total droplet volume, and $L$ the characteristic dissipation length scale, which in this model is selected as the height of the droplet splat $h$. In the model by Chandra \& Avedisian [29], $W$ is used in an energy balance $E_{k}+E_{s}=W+E_{s}^{\prime}$, where $E_{k}$ is kinetic energy, and $E_{s}$ and $E_{s}^{\prime}$ are surface energies before and after impact, respectively. However, as shown by Pasanideh-Fard et al. [1] and our results, this model strongly overpredicts the maximum spreading radius.

The model was revisited by Pasandideh-Fard et al. [1]. Their numerical simulations suggested that the dissipation is taking place in a thin boundary layer within the expanding droplet, implying that the characteristic length scale $L$ in the above eq. 3.1 is not the pancake thickness $h$ but has to be replaced by the Prandtl-Blasius boundary layer thickness $\delta[1]$ :

$$
\delta=2 \frac{D_{0}}{\sqrt{\mathrm{Re}}}
$$

finally resulting in the following equation for $D_{\max } / D_{0}$ :

$$
D_{\text {max }} / D_{0}=\sqrt{\frac{\mathrm{We}+12}{3(1-\cos \theta)+4(\mathrm{We} / \sqrt{\mathrm{Re}})}} .
$$



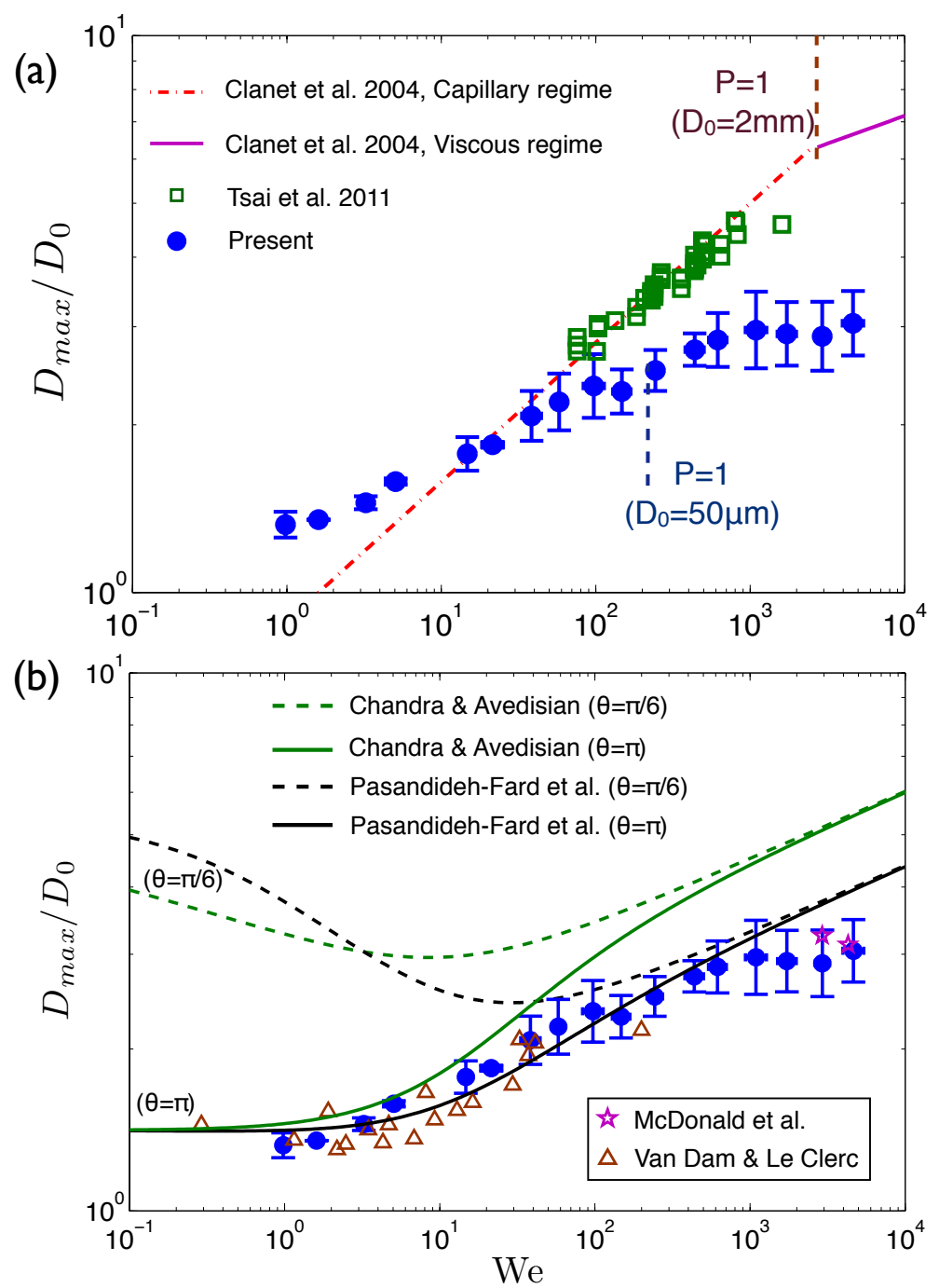

Figure 3.4: The normalized maximum spreading $D_{\max } / D_{0}$ vs. We. (a) Solid circles: the present microdroplet data. Open squares: mm-sized droplets impact on superhydrophobic surfaces from Tsai et al. [16] . Dash-dot line: the capillary model by Clanet et al. [10]. Solid line: the viscous model by Clanet et al. [10]. The short vertical dashed lines show the transitional Weber number $\mathrm{We}_{\mathrm{t}}$ at $P=1$ for a $2 \mathrm{~mm}$-sized droplet and for a $50 \mu \mathrm{m}$ droplet. (b) Solid circles: the present microdroplet data. Stars: data from Mc Donald et al. [5] with impact of molten metal microdroplets. Open triangles: low-speed microdroplet impact experiments by Van Dam \& Le Clerc [8]. Lines: Model by Chandra \& Avedisian [29] (darkgreen lines) and by Pasandideh-Fard [1] model (black lines), evaluated for the initial diameter $D_{0}=50 \mu \mathrm{m}$ and contact angles $\pi / 6$ (dashed lines) and $\pi$ (solid lines). 
Here, $\theta$ is the contact angle. At low impact velocities $(\mathrm{We} \lesssim 10)$ this model saturates, as the droplet impact can be considered to be effectively static and $E_{k}$ and $W$ vanish.

In figure 3.4 (b), the measured maximum spreading for the microdroplet impacts is plotted versus the Weber number. The maximum spreading is measured at the moment when the deformation of the droplet is maximum before it wets the surfaces, as shown in figure $3.3(\mathrm{a}, \mathrm{b})$. At low velocity, the present data saturates around a spreading of $\sim 1.3$ times the initial diameter, which has a good overlap with previous low-speed microdroplet impact experiments [8]. At high velocities, spreading of only $\sim 3$ times the initial diameter is found for even the fastest droplets. This is consistent with (even faster) impact of molten metal microdroplets [5], even though the liquid properties were very different from our experiments.

Figure 3.4 (b) is complemented with the models of Chandra \& Avedisian [29] and Pasandideh-Fard [1], evaluated for an initial diameter of $D_{0}=50 \mu \mathrm{m}$. We first examine the maximum spreading calculated from these models for two different contact angles, i.e. $\theta=\pi / 6$ and $\pi$. The dashed lines in figure 3.4 (b) clearly show that the contact angle of $\pi / 6$ results in a much larger spreading factor for both models as compared to the experimental data. As discussed above, an air layer is present between the spreading drop and the solid surface before the droplet wets the surface. First, it was shown that decreasing the air pressure can prevent splashing [24]. Later, the temporal evolution of the air layer has been experimentally measured [22] and investigated numerically [21]. The existence of the air layer implies a "contact angle" which would be best described by $\theta=\pi$. As shown with the solid lines in figure 3.4 (b), this approach remarkably decreases the deviations between the experiments and the results of both models. A good agreement is found between the microdroplet data and the Pasandideh-Fard model [1] up to $\mathrm{We} \approx 10^{3}$. This indicates the importance of a finite boundary layer thickness in the dissipation of spreading droplets in the present parameter regime. For We $>10^{3}$, the increasing trend of $D_{\max } / D_{0}$ versus We seems to saturate. This finding is consistent with Ref. [9], and will be studied in future work.

\subsection{Conclusions}

The impact of water microdroplets on a smooth solid surfaces is investigated experimentally. By using a new droplet-generating device, impact events were created at velocities from 1 to $100 \mathrm{~m} / \mathrm{s}$ and droplet diameters between 12 and $100 \mu \mathrm{m}$. This parameter regime covers the transition between surface tension- and viscositydominated spreading of the droplet. For all impact events, no splashing is observed. The maximum spreading radius was compared to several models. The model by 
Pasandideh-Fard et al. [1] performs best, indicating that boundary layer dynamics play a key role in droplet spreading. In addition, we find that an initial contact angle of 180 degrees should be used as input value. This confirms the presence of an air layer under the impacting droplet.

\section{References}

[1] M. Pasandideh-Fard, Y. M. Qiao, S. Chandra, and J. Mostaghimi, Capillary effects during droplet impact on a solid surface, Phys. Fluids 8, 650 (1996).

[2] S. Mitra, Breakup process of plane liquid sheets and prediction of initial droplet size and velocity distributions in sprays, $\mathrm{PhD}$ thesis (University of Waterloo) (2001).

[3] J. Blaisot and J. Yon, Droplet size and morphology characterization for dense sprays by image processing: application to the Diesel spray, Exp. Fluids 39, 977 (2005).

[4] K. Haller, Y. Ventikos, D. Poulikakos, and P. Monkewitz, Computational study of high-speed liquid droplet impact, J. Appl. Phys. 92, 2821 (2002).

[5] A. McDonald, M. Lamontagne, C. Moreau, and S. Chandra, Impact of plasma-sprayed metal particles on hot and cold glass surfaces, Thin Solid Films 514, 212 (2006).

[6] M. Rein, Phenomena of liquid drop impact on solid and liquid surfaces, Fluid Dyn. Res. 12, 61 (1993).

[7] A. L. Yarin, Drop impact dynamics: Splashing, Spreading, Receding, Bouncing..., Annu. Rev. Fluid Mech. 38, 159 (2006).

[8] D. van Dam and C. L. Clerc, Experimental study of the impact of an ink-jet printed droplet on a solid substrate, Phys. Fluids 16, 3403 (2004).

[9] L. Cheng, Dynamic spreading of drops impacting onto a solid surface, Industrial and Engineering Chemistry Process Design and Development 16, 192 (1977).

[10] C. Clanet, C. Béguin, D. Richard, and D. Quéré, Maximal deformation of an impacting drop, J. Fluid Mech. 517, 199 (2004).

[11] R. Bhola and S. Chandra, Parameters controlling solidification of molten wax droplets falling on a solid surface, J. of Mater. Sci. 34, 4883 (1999).

[12] R. E. Ford and C. G. L. Furmidge, Impact and spreading of spray dropls on foliar surfaces, Wetting, Society of Chemical Industry (London), Monograph 25417 (1967).

[13] H. Fukanuma and A. Ohmori, Behavior of molten droplets impinging on flat surfaces, Proc. of the 7th Natational Thermal Spray Conference 563 (1994).

[14] H. Marmanis and S. T. Thoroddsen, Scaling of the fingering pattern of an impacting drop, Phys. Fluids 8, 1344 (1996).

[15] C. D. Stow and M. G. Hadfield, An experimental investigation of fluid flow resulting from the impact of a water drop with an unyielding dry surface, Proceedings of the Royal Society of London. A. Mathematical and Physical Sciences 373, 419 (1981).

[16] P. Tsai, M. W. Hendrix, R. M. Dijkstra, L. Shui, and D. Lohse, Microscopic structure influencing macroscopic splash at high Weber number, Soft Matter 7, 11325 (2011).

[17] T. Tran, H. J. J. Staat, A. Prosperetti, C. Sun, and D. Lohse, Drop impact on superheated surfaces, Phys. Rev. Lett. 108, 036101 (2012). 
[18] Y. Tagawa, N. Oudalov, C. W. Visser, I. R. Peters, D. van der Meer, C. Sun, A. Prosperetti, and D. Lohse, Highly focused supersonic microjets, Phys. Rev. X, in press (2012).

[19] C. T. Chin, C. Lancee, J. Borsboom, F. Mastik, M. E. Frijlink, N. de Jong, M. Versluis, and D. Lohse, Brandaris 128: A digital 25 million frames per second camera with 128 highly sensitive frames, Rev. Sci. Instrum. 74, 5026 (2003).

[20] A. van der Bos, A. Zijlstra, E. Gelderblom, and M. Versluis, iLIF: illumination by Laser-Induced Fluorescence for single flash imaging on a nanoseconds timescale, Exp. Fluids 51, 1283 (2011).

[21] M. Mani, S. Mandre, and M. P. Brenner, Events before droplet splashing on a solid surface, J. Fluid Mech. 647, 163 (2010).

[22] R. C. A. van der Veen, T. Tran, D. Lohse, and C. Sun, Direct measurements of air layer profiles under impacting droplets using high-speed color interferometry, Phys. Rev. E 85, 026315 (2012).

[23] W. Bouwhuis, R. C. A. Van der Veen, T. Tran, D. L. Keij, K. G. Winkels, I. R. Peters, D. Van der Meer, C. Sun, J. H. Snoeijer, and D. Lohse, Maximal air bubble entrainment at liquid drop impact, arXiv:1205.4761 (2012).

[24] L. Xu, W. Zhang, and S. Nagel, Drop splashing on a dry smooth durface, Phys. Rev. Lett. 94, 184505 (2005).

[25] M. M. Driscoll and S. R. Nagel, Ultrafast interference imaging of air in splashing dynamics, Phys. Rev. Lett. 107, 154502 (2011).

[26] S. Mandre and M. P. Brenner, The mechanism of a splash on a dry solid surface, J. Fluid Mech. 690, 148 (2012).

[27] S. Thoroddsen, T. Etoh, K. Takehara, N. Ootsuka, and A. Hatsuki, The air bubble entrapped under a drop impacting on a solid surface, J. Fluid Mech. 545, 203 (2005).

[28] J. Eggers, M. Fontelos, C. Josserand, and S. Zaleski, Drop dynamics after impact on a solid wall: Theory and simulations, Phys. Fluids 22, 062101 (2010).

[29] S. Chandra and C. T. Avedisian, On the collision of a droplet with a solid surface, Proceedings: Mathematical and Physical Sciences 432, 13 (1991).

[30] S. E. Bechtel, D. B. Bogy, and F. E. Talke, Impact of a liquid drop against a flat surface, IBM Journal of Research and Development 25, 963 (1981).

[31] J. Madejski, Solidification of droplets on a cold surface, Int. J. Heat Mass Transfer 19, 1009 (1976).

[32] I. V. Roisman, R. Rioboo, and C. Tropea, Normal impact of a liquid drop on a dry surface: model for spreading and receding, Proceedings of the Royal Society of London. Series A: Mathematical, Physical and Engineering Sciences 458, 1411 (2002). 


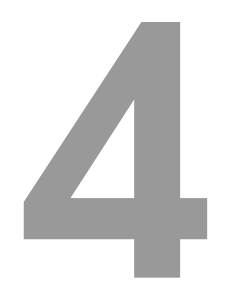

\section{Dynamics of high-speed micro-droplet impact: numerical simulations and experiments at frame rates beyond $10^{7} \mathrm{fps}^{*} \dagger$}

Technologies including (3D-)(bio-)printing, diesel engines, laser-induced forward transfer, and spray cleaning require optimization and therefore understanding of micrometer-sized droplets impacting at velocities beyond $10 \mathrm{~m} / \mathrm{s}$. However, as yet, this regime has hardly been addressed. Here we present the first time-resolved experimental investigation of microdroplet impact at velocities up to $50 \mathrm{~m} / \mathrm{s}$, on hydrophilic and -phobic surfaces at frame rates exceeding $10^{7}$ frames per second. A novel method to determine the 3D-droplet profile at sub-micron resolution at the same frame rates is presented, using the fringe pattern observed from a bottom view. A numerical model, which is validated by the side- and bottom-view measurements, is employed to study the viscous boundary layer inside the droplet and the development of the rim.

The spreading dynamics, the maximal spreading diameter, the boundary layer thickness, the

\footnotetext{
${ }^{*}$ Submitted as: C.W. Visser, P.E. Frommhold, S. Wildeman, R. Mettin, D. Lohse, and C. Sun.

${ }^{\dagger}$ Experimental setup (in part), experiments (in part), data processing of experiments and simulations, and first draft by CWV.
} 
rim formation, and the air bubble entrainment are compared to theory and previous experiments. In general, the impact dynamics are equal to millimeter-sized droplet impact for equal Reynolds- Weber- and Stokes numbers. Using our numerical model, effective scaling laws for the progression of the boundary layer thickness and the rim diameter are provided. The boundary layer thickness develops according to $\delta_{B L} \sim D_{0} / \sqrt{\operatorname{Re}} \cdot(t / \tau)^{0.45}$ and the diameter of the rim develops according to $D_{\text {Rim }} \sim D_{0} / \sqrt{\mathrm{We}} \cdot(t / \tau)^{0.68}$. These scalings differ from previously assumed, but never validated, values. Finally, no splash is observed, at variance with many predictions but in agreement with models including the influence of the surrounding gas. This confirms that the ambient gas properties are key ingredients for splash threshold predictions.

\subsection{Introduction}

Fast impact of small-scale droplets is a key phenomenon in applications such as diesel engines [1, 2], thermal spraying [3], spray cleaning [4], and novel manufacturing technologies based on ink-jet printing technology [5-7] or laser-induced forward transfer [8]. However, the impact of these droplets is often poorly controlled, as it has hardly been visualized. The associated industrial problems include (but are not limited to) defects in metal electronics printing [3,9], reduced cell survival in spray treatment of burn wounds [10], damage on semiconductor wafers [11], and limited steel strip surface quality [12]. To achieve a better understanding and thereby allow for improved process control, in this article we study the impact of fast, micro-scale droplets on a dry, smooth surface.

This is a challenging task, since for a droplet with diameter $D_{0}=50 \mu \mathrm{m}$ impacting at $50 \mathrm{~m} / \mathrm{s}$, the impact time scale is $\tau=D_{0} / V_{0} \sim 1 \mu \mathrm{s}$. As this is comparable to the shutter time of high-quality fast cameras, these are still too slow to resolve the details of impact. Much shorter exposure times can be achieved using flash photography [13]. However, with flash photography, only one or two frames per impacting droplet can be captured. Therefore, to obtain multiple frames of the impact using the flash approach, multiple droplet impacts with excellent reproducibility are required. So far, sufficiently reproducible droplets could only be made up to $20 \mathrm{~m} / \mathrm{s}$ [14-16]. As a result, single-droplet impact at high velocity $\left(V_{0}>10 \mathrm{~m} / \mathrm{s}\right)$ and small diameters $\left(D_{0}<100 \mu \mathrm{m}\right)$ has hardly been visualized in detail, despite the vast interest for this subject in the context of the technologies mentioned.

In contrast, major progress has recently been made for mm-sized droplet impact (for background see reviews [34, 35]). We first provide a short overview of 

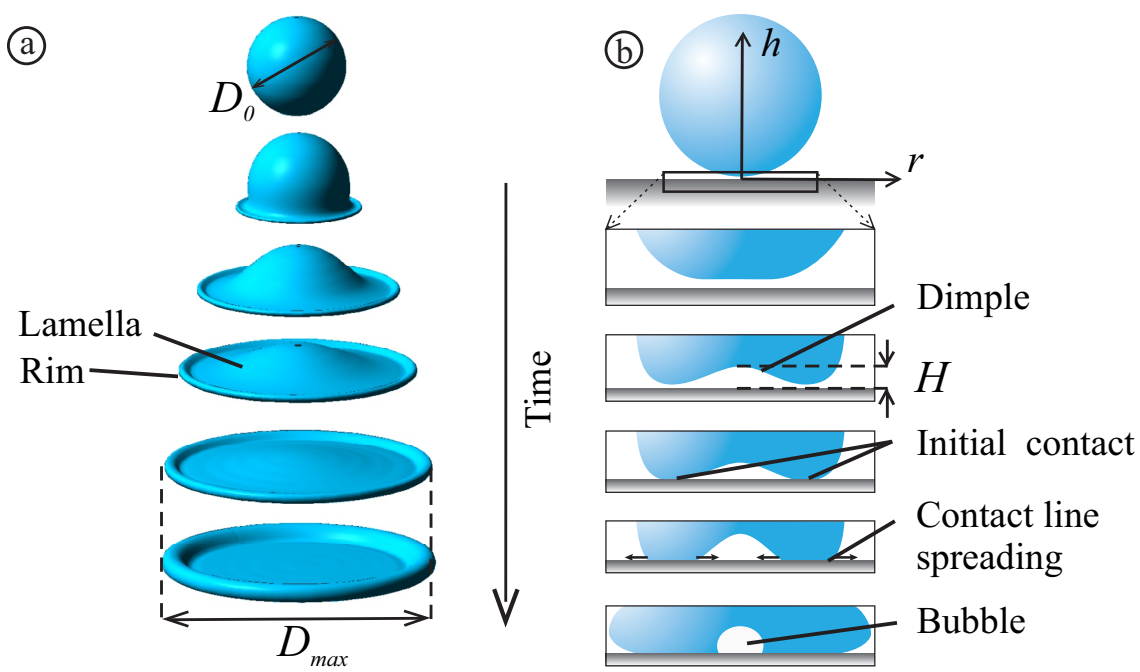

Figure 4.1: Overview of the different stages of droplet impact. (a) A droplet with diameter $D_{0}$ and velocity $V_{0}$ is impacting onto a flat smooth surface (only liquid shown). During its spreading into a lamella, a rim is formed. (b) Droplet-substrate interaction in the initial stage of impact, after [17]. The rectangle in the top image is enlarged in the subsequent images, showing snapshots of the cross-section of the droplet. Initially the droplet flattens and forms a dimple, due to compression of the ambient gas between the droplet and the surface. After the initial contact is made, the air under the dimple is entrained and contracts into a bubble.

these findings, as they provide the required context for our results on micro-droplets. As schematically shown in figure 4.1(a), the impacting droplet spreads into a thin lamella until a maximum diameter is reached. The spreading of droplets was shown to be limited by either surface tension or viscosity [21]. The formation of a viscous boundary layer was shown to be crucial [26, 36, 37], and has been included in several experimentally validated models. A self-similar profile of the droplet during spreading was theoretically predicted [36] and recently validated by 3D-measurements of the droplet surface [24]. These articles consistently showed that the spreading of an impacting mm-sized droplet can be described sufficiently by the Reynolds number $\operatorname{Re}=\rho D_{0} V_{0} / \mu$ with liquid density $\rho$, dynamic viscosity $\mu$, and the Weber number $\mathrm{We}=\rho D_{0} V_{0}^{2} / \sigma$, with surface tension $\sigma$. In addition, the droplet-air interaction was found to be crucial in the initial stages of droplet impact [17, 19, 32, 38-41], as schematically shown in figure 4.1b. Prior to impact, the gap between the droplet and the surface becomes extremely slender, and the escape of the gas from this gap is strongly limited by viscous dissipation in the gas. The trapped gas forms a film between the droplet and the substrate. This film causes a "dimple" in the droplet surface 

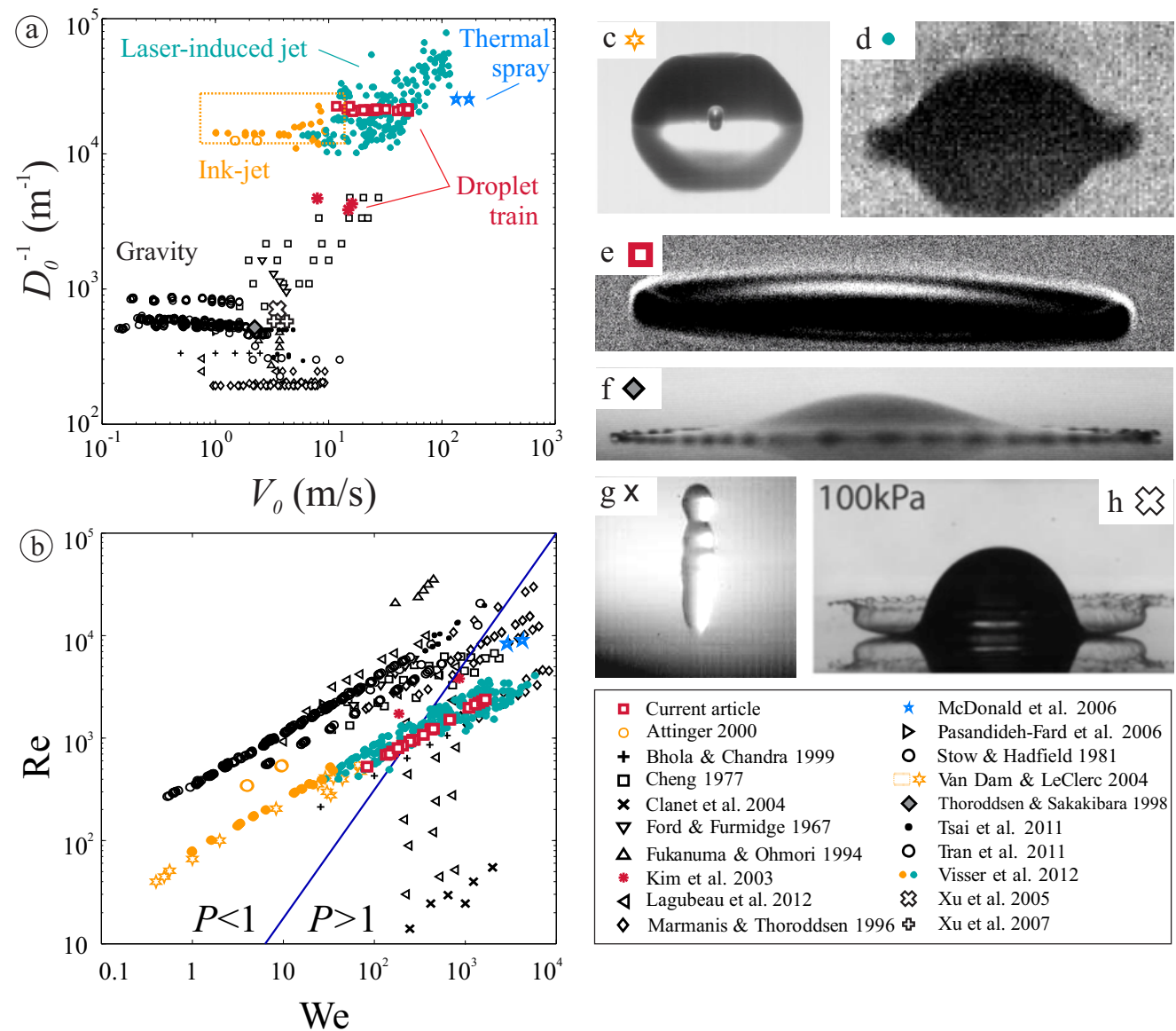

口 Current article

- Attinger 2000

+ Bhola \& Chandra 1999

口 Cheng 1977

× Clanet et al. 2004

$\boldsymbol{\nabla}$ Ford \& Furmidge 1967

$\Delta$ Fukanuma \& Ohmori 1994

* Kim et al. 2003

$\triangleleft$ Lagubeau et al. 2012

$\diamond$ Marmanis \& Thoroddsen 1996 $\star \quad$ McDonald et al. 2006

$\triangleright$ Pasandideh-Fard et al. 2006

○ Stow \& Hadfield 1981

- iz Van Dam \& LeClerc 2004

$\diamond$ Thoroddsen \& Sakakibara 1998

- Tsai et al. 2011

- Tran et al. 2011

Visser et al. 2012

\Xu et al. 2005

Xu et al. 2007

Figure 4.2: Overview of experimental work on droplet impact, from refs. $[3,5,14,15,18$ 33], showing the phase of inverse diameter versus velocity (a) and the Reynolds number versus the Weber number (b). The line indicates the separation between viscosity- and surface tension-dominated regimes (under versus above the line, respectively) of droplet spreading as proposed in ref. [21], and discussed in section 4.3.6. Figures (c) to (f) illustrate the wide range of outcomes of droplet impact. Microdroplet impact on a flat surface is shown for water at room temperature in (c), (d), and (e), illustrating the entrainment of an air bubble at low-velocity impact (c) [14], the limited resolution obtained by high-speed camera imaging at high-velocity impact (d) [31], and the development of a rim (e) (current work). Figures (f) to $(\mathrm{h})$ are obtained for $\mathrm{mm}$-sized impact, illustrating rim instabilities (f) [28], bouncing (g) [21], and splashing (h) [32]. 
due to the pressure building under the falling droplet, and prevents immediate contact between the droplet and the substrate, see figure 4.1b. Due to the dimple, the (axisymmetric) minimum of the air film thickness is found off-axis. Eventually, continued thinning of the air film results in contact between the droplet and the substrate, and a wetting front starts running inwards and outwards from the initial, ring-shape contact (indicated by small arrows). The dynamics of the entrapped air can be complex [42-44], but usually the air entrained by the dimple forms a small bubble inside the droplet $[14,44,45]$. The dimple- and bubble-sizes were quantified experimentally, numerically and theoretically, using the Reynolds-, Weber-, and Stokes-numbers as control parameters, with Stokes number $\mathrm{St}=\rho D_{0} V_{0} /\left(2 \mu_{g}\right)$, in which $\mu_{g}$ is the gas viscosity [19].

Can these results for mm-sized droplets provide any insight for micro-scale impacts? As discussed, the parameter space of the impact problem is huge, with the droplet diameter, velocity, viscosity, surface tension, and ambient pressure being the most relevant parameters. A cross-section of this parameters space (the diametervelocity sub-space) is shown in figure 4.2a. Even in this limited cross section the phenomenology of the impact problem is extremely rich. Consider for example (c) the entrainment of an air bubble by an impacting microdroplet [14]; (d) a microdroplet during its impact on a flat surface [31]; (e) the development of a thick rim, which for different impact conditions can develop an instability shown in figure 4.2(f) [33, 46]; (g) a droplet boucing back from a superhydrophobic surface [21, 47, 48]; and (h) splashing of an ethanol droplet on a flat surface [32]. Assuming the relevance of certain forces and processes, one achieves a dimensionless representation of the parameter space as shown in figure 4.2 (b) (in terms of the Weber- and Reynolds numbers; the Stokes number is the third dimensionless parameter). This shows that the dimensionless control parameters for microdroplet impact coincide to experiments performed for mm-sized droplets (which are performed at a higher viscosity and lower velocity as compared to micrometer-sized droplets, see for example refs. [18, 25]). If the impact problem is scale-invariant, this means that the mm-droplet experiments can be used to obtain accurate predictions for the micro-scale experiments. However, it is not obvious that this scale-invariance must hold. For example the wetting properties of sessile macro-and microdroplets can be rather different [49], due to different contact angles at the macro-and micro-scale. Also, the other dimensionless parameters may become relevant at small scales. Thus, it is a priori unclear whether different dimensional representations of the same point in the dimensionless subspace of the impact problem indeed show the same behavior - this depends on whether the assumptions regarding the relevant processes and forces are correct.

In this work we provide a comprehensive analysis of microdroplet impact em- 
ploying novel experimental and numerical methods discussed in section 4.2. We visualize for the first time the impact of fast $\left(V_{0} \gg 10 \mathrm{~m} / \mathrm{s}\right)$ microdroplets at high resolutions in section 4.3.1, using an interferometric approach to extract the 3D-profile of the droplet surface during spreading at sub-micron resolution. In the subsequent sections we use the numerics and experiments to analyze the spreading dynamics, the air entrainment, the boundary layer flow, the development of a rim, the maximal spreading, and the splashing threshold, and compare these results to those obtained for mm-scale droplets.

\subsection{Droplet generation, visualization, and modeling}

\subsubsection{Droplet generation}

To obtain high-resolution images of fast droplet impact on a glass substrate, we create highly reproducible microdroplets (both temporally and spatially), analogous to what was done in refs. [15, 50, 51]. Figure 4.3 shows an image of our setup. We first produce a liquid jet by pumping distilled water through a $\sim 20 \mu \mathrm{m}$ orifice. Using an acoustic element the jet is vibrated at a controlled frequency, forcing Rayleigh break-up of the jet into a train of monodisperse droplets. By applying a positive electrical pulse of duration $\sim \tau$ to the charging electrode, the tip of the jet is temporarily charged negatively at the moment of break-up, and a charged droplet is created. When traveling in the electric field between the deflection plates, this droplet is slightly deflected $\left(\theta<1^{\circ}\right)$. Catching away the droplet train then results in impact of a single droplet on the impact plate, at a velocity controlled by the jet velocity. Three surfaces were used to assess the influence of the macroscopic advancing contact angle $\theta$ for droplet spreading: Clean glass, with $\theta=23^{\circ}$; RainX, a windshield-repellency coated glass surface (brand: RainX) with $\theta=90^{\circ}$, and a hydrophobized aluminium oxide surface [52] with $\theta=135^{\circ}$. To prevent charge build-up, the capillary tube, the impact plate, and the reservoir were grounded. The parameter space that was scanned with the current setup is shown in figure 4.2a (all experiments were performed in air at standard conditions).

\subsubsection{Droplet visualization}

To visualize the droplet from the side, a double-shutter camera (Sensicam QE, PCO AG, Germany) was used. A 6 ns pulse from dual-flash ND:YAG laser (Quantel, France) provided bright-field illumination. To prevent fringes, coherence was removed by inserting a fluorescent plate in front of the laser. A bottom view was 


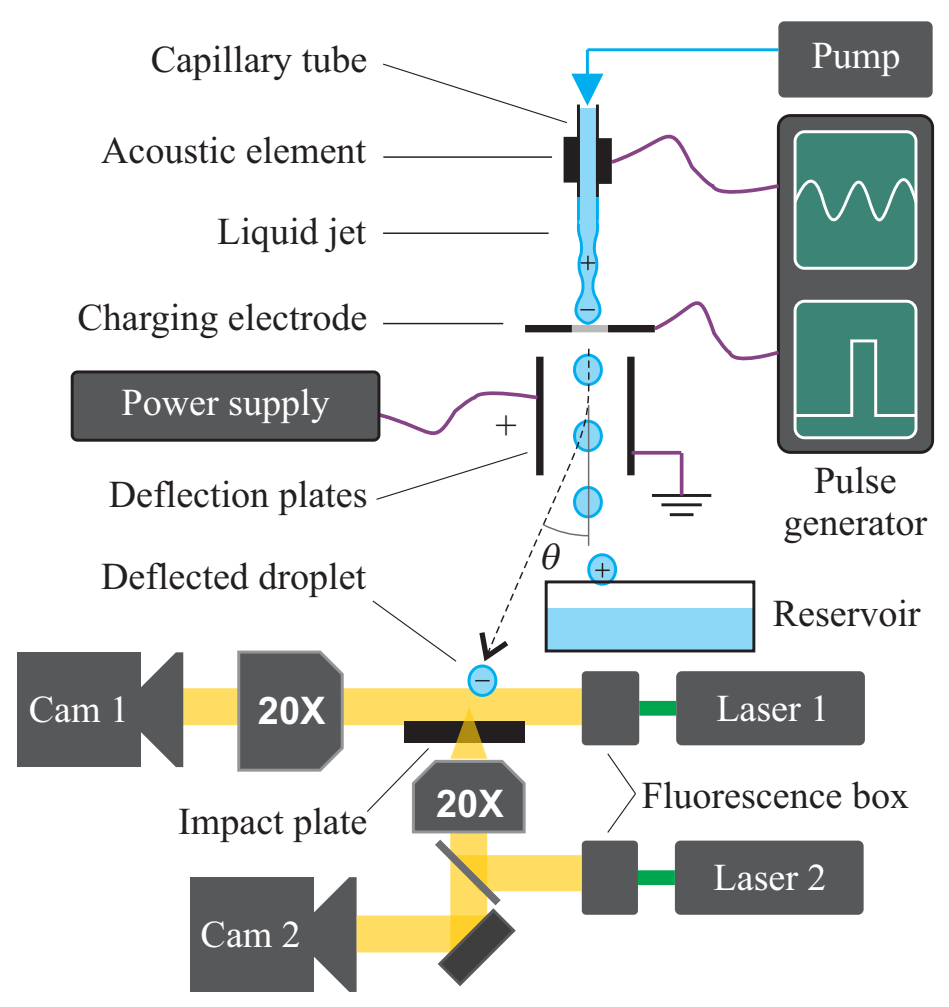

Figure 4.3: Setup. A liquid jet is created by pumping the liquid through a $20 \mu \mathrm{m}$-sized orifice. The jet is acoustically oscillated, to force its break-up into a train of monodisperse droplets. A high-voltage pulse is applied to a charging electrode, just before break-up into a droplet. This charges the jet tip, resulting in a single, charged droplet. This droplet it deflected from the non-charged droplet train by an electrical field. Catching away the droplet train then results in a single, highly reproducible impact on the impact plate. Synchronized side- and bottom views are used for visualization. 
realized similar to the side view. A half-mirror was added to achieve co-axial, darkfield illumination. The camera- and laser timings were synchronized for the side- and bottom-views. All temporal delays were controlled using pulse generators (Berkeley Nucleonics Corporation, model 575).

\subsubsection{Determination of droplet size, velocity, and moment of impact}

For each experimental setting, the droplet size and velocity were determined as displayed in figure 4.4. From two side-view images of the in-flight droplet, indicated as $1 \mathrm{~A}$ and $1 \mathrm{~B}$ in figure $4.4 \mathrm{a}$, the droplet position was obtained by automated image analysis in MATLAB ( MathWorks Inc., Natick, MA, 2013). As the delay between the frames $\Delta t$ is known, the spatial difference $\Delta y_{1}$ readily provides the droplet velocity $V=\Delta y_{1} / \Delta t$. For each experimental setting, at least 10 image pairs were processed to obtain a velocity histogram as plotted in figure $4.4 \mathrm{~b}$. From this histogram, the median velocity provides the per-experiment velocity $V_{0}$. In all cases, the velocity has a typical standard deviation of $0.01 V_{0}$. The droplet diameter was obtained from frame $1 \mathrm{~A}$ and $1 \mathrm{~B}$ of the velocity calibration measurements and each first frame of the impact experiments (frame 2A), resulting in $\sim 150$ diameter measurements for each experimental setting. A histogram of the droplet diameter is plotted in figure $4.4 \mathrm{~d}$. Again the median provides $D_{0}$ with a typical standard deviation of $0.01 D_{0}$.

An example impact experiment is shown in figure 4.4c, where frame B shows a clear image of the impacting droplet. However, the arrival time of the droplet has an uncertainty of $\sim 1 \mu$ s due to the $1 \%$ uncertainty in the droplet velocity. This is similar to the typical impact time $\tau$, thus a more accurate time determination is required to resolve the impact dynamics. Therefore, for each image pair, we first calculate the time difference between the first droplet-substrate contact and frame A as $t_{A}=-\tau / 2-\Delta y / V_{0}$, with $\Delta y$ the distance between the droplet and the substrate. This yields a negative value, as time $t=0 \mathrm{~s}$ is defined as the moment of impact. The time of frame B is $t_{B}=t_{A}+\Delta t$, with $\Delta t$ the frame-to-frame delay as set with the pulse generator. The per-frame time uncertainty using this method is $100 \mathrm{~ns} \ll \tau$, i.e. sufficient for our measurements. To scan the temporal axis of the full impact, this procedure is repeated $\sim 150$ times for each experimental setting, with different delays. High-speed movies of the side- and bottom view are constructed for each experiment (example movies are provided for control parameters listed in table S1 and figure S1). 
(a)

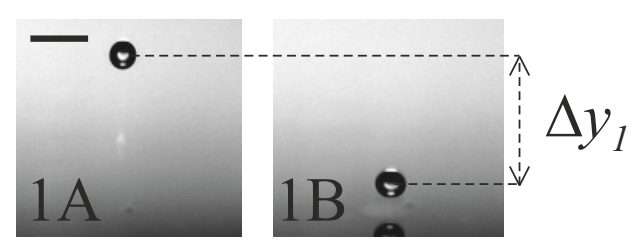

(c)

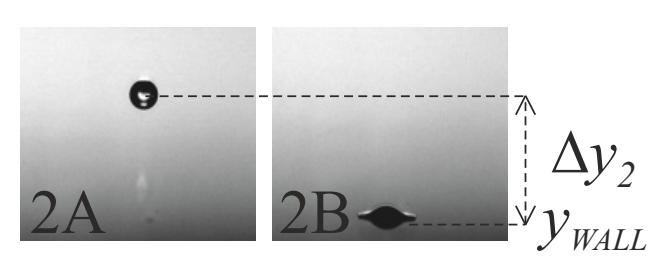

(e)

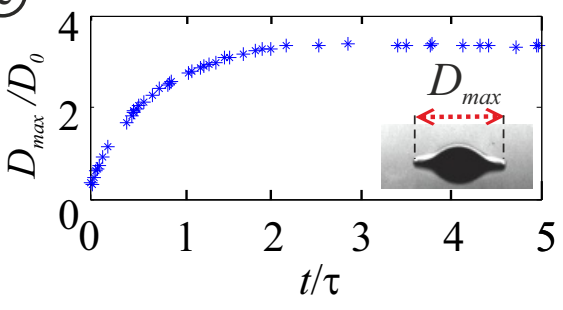

(b)

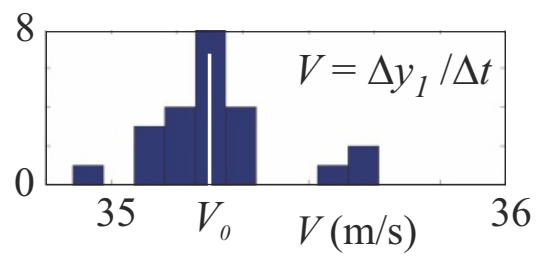

(d)

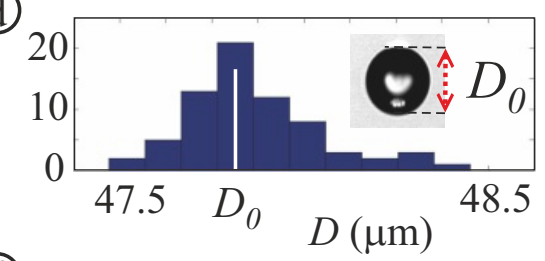

(f)

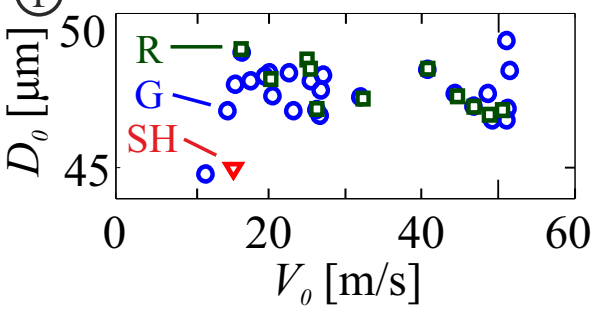

Figure 4.4: Determination of droplet size and velocity. All images shown are for equal droplet generation settings. (a) Two in-flight frames are used to determine the droplet translation $\Delta y_{1}$. The translation is used to obtain the droplet velocity. The scale bar indicates $100 \mu \mathrm{m}$. (b) Velocity histogram. The median provides the impact velocity $V_{0}$. Here and in (d), note the scale of the velocity axis: the distribution is very narrow. (c) Droplet captured in-flight (frame 2A) and during spreading (frame 2B). Frame A is used to collect the diameter $D$ for each experiment. (d) Diameter histogram. The median provides the droplet diameter for each experimental setting, $D_{0}$. (e) Example curve of $D_{\max } / D_{0}$ is plotted versus time. The maximum spreading diameter $D_{\max }$ is determined from frame 2B. (f) Parameter space of $D_{0}$ and $V_{0}$. The markers indicate the surface type $\left(\mathrm{G}\right.$ : Glass $\left(\theta=23^{\circ}\right)$, R: RainX $\left(\theta=90^{\circ}\right), \mathrm{SH}$ : Superhydrophobic $\left(\theta=135^{\circ}\right)$ ). 


\subsubsection{Numerical methods}

Simulations were done using the open-source flow solver Gerris [53], which numerically solves the incompressible Navier-Stokes equation on a non-uniform flowadaptive grid. The interface-tracking volume of fluids (VOF) implementation in Gerris makes it well-suited for problems involving two phases with a high density ratio and interfacial tension (water and air). In our axisymmetric simulations we take a uniform grid inside the droplet with typically 340 points across the diameter (i.e. $0.15 \mu \mathrm{m}$ grid cell size for a $D_{0}=50 \mu \mathrm{m}$ droplet). The interface of the drop is adaptively discretized, ensuring that the curvature per cell does not exceed $5 \%$ of the droplet radius. The minimum resolution is limited to the resolution inside the droplet. Outside the droplet the grid is adapted according to the norm of the vorticity, with a maximum grid size equal to that of the droplet. The size of the (squared) simulation domain is 3 droplet diameters, with outflow boundary conditions on the top and left edge, no-flow conditions on the bottom edge and symmetry conditions on the right edge (which represent the z-axis along which the droplet approaches the surface). During the downward impact, the air-layer between the droplet and the bottom surface is given extra resolution to prevent the VOF-interface from coalescing with the wall (which also allows for bigger time steps). In most simulations there remains a minimum gap of about $20 \%$ of a droplet grid cell (i.e. $30 \mathrm{~nm}$ ) between the bottom of the domain and the droplet interface. As the viscosity and density do not sharply change over the interface, this means that the droplet is effectively in contact with the no-slip bottom edge at these points (with a $180^{\circ}$ contact angle at the contact line).

Example results of the model are shown in figures 4.1a and 4.5. The model is validated versus our measurements in figures 4.6, 4.8, 4.9, and 4.14, as will be discussed in the corresponding subsections of this article.

\subsection{Results and discussion}

\subsubsection{Droplet profile}

From the side- and bottom view snapshots shown in figure 4.6, we can identify different stages of the droplet impact. Image 4.6a shows the droplet just before impact. Subsequently, the droplet spreads over the surface into a lamella. As visible in images $4.6 \mathrm{c}$ to $4.6 \mathrm{e}$, a rim is formed at the edge of the sheet. After reaching its maximal diameter, the droplet retracts (for hydrophobic surfaces) or the contact line pins and the droplet comes to rest after some capillary oscillations. As we focus on the impact dynamics, these stages are not shown here.

Prior to impact, the bottom view reveals faint fringes (figure 4.6a). These fringes 


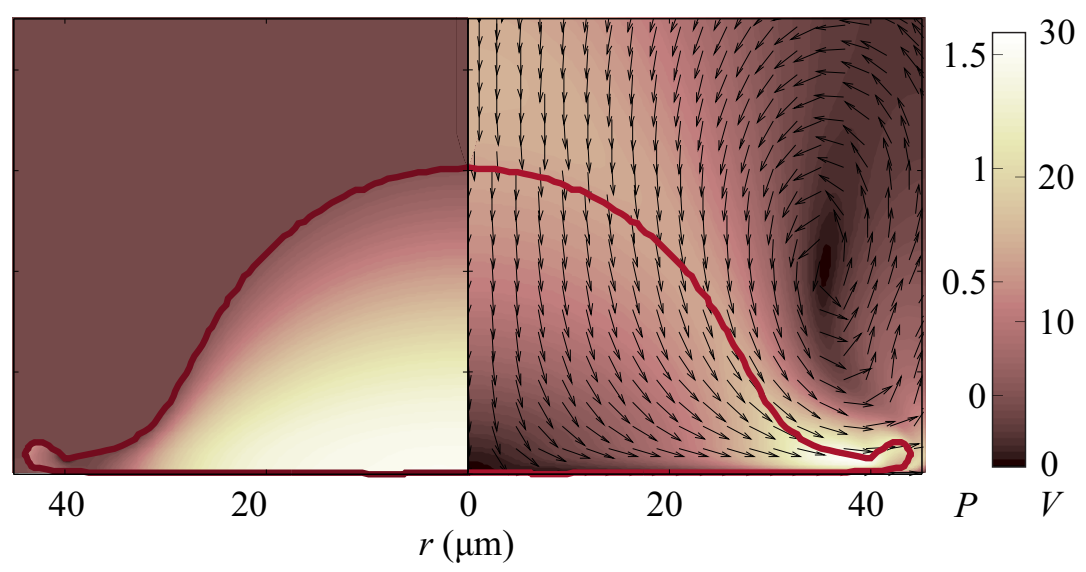

Figure 4.5: Simulated droplet impact. Left half: Pressure field; the color map indicates the pressure $P$ in $10^{5} \mathrm{~Pa}$. Right half: Velocity field, in which the arrows and the colors respectively indicate the flow direction and flow velocity (in $\mathrm{m} / \mathrm{s}$ ). Simulation parameters: $D_{0}=50 \mu \mathrm{m}$ and $V_{0}=17 \mathrm{~m} / \mathrm{s}$, corresponding to $\mathrm{We}=195$ and $\mathrm{Re}=836$. The snapshot is taken at $t / \tau=0.42$.

are due to the air film between the droplet and the substrate, and will be discussed in subsection 4.3.3. In the later stages of droplet spreading, the bottom view again reveals clear fringes (figure $4.6 \mathrm{~d}$ and e). These fringes are due to interference between the top droplet-air interface and the glass or droplet bottom.

We now analyze the droplet surface profile from the fringes in figure 4.6d, which have been magnified in figure 4.7a. After radial averaging, the black and white fringes result in the intensity profile shown in figure $4.7 \mathrm{~b}$. The local minima and maxima of this curve were automatically detected using MATLAB and plotted as open and closed circles, respectively. As the droplet thickness is monotonically decreasing in height within the lamella radius (indicated as $R_{L}$ in figure $4.6 \mathrm{~d}$ ), each black-to-white fringe transition is associated to a quarter-wavelength step down in height. The resulting profile is indicated by the dotted curve in figure $4.7 \mathrm{c}$, where the minimum is set to zero as an initial value. However, the fringes only provide the slope of teh droplet surface, thus the actual lamella thickness remains to be determined.

To find the actual lamella thickness we calculate the volume contained withing the profile plotted in figure $4.7 \mathrm{c}$, by fitting a third-order polynomial to it (black line) and integrating the resulting curve assuming axisymmetry. This volume, corresponding to area " $\mathrm{A}$ " in figure $4.7 \mathrm{c}$, is much smaller than the actual droplet volume. This is not surprising, as a part of the droplet volume is contained in the rim, and the lamella must have a non-vanishing thickness everywhere. If we assume that the rim contains 

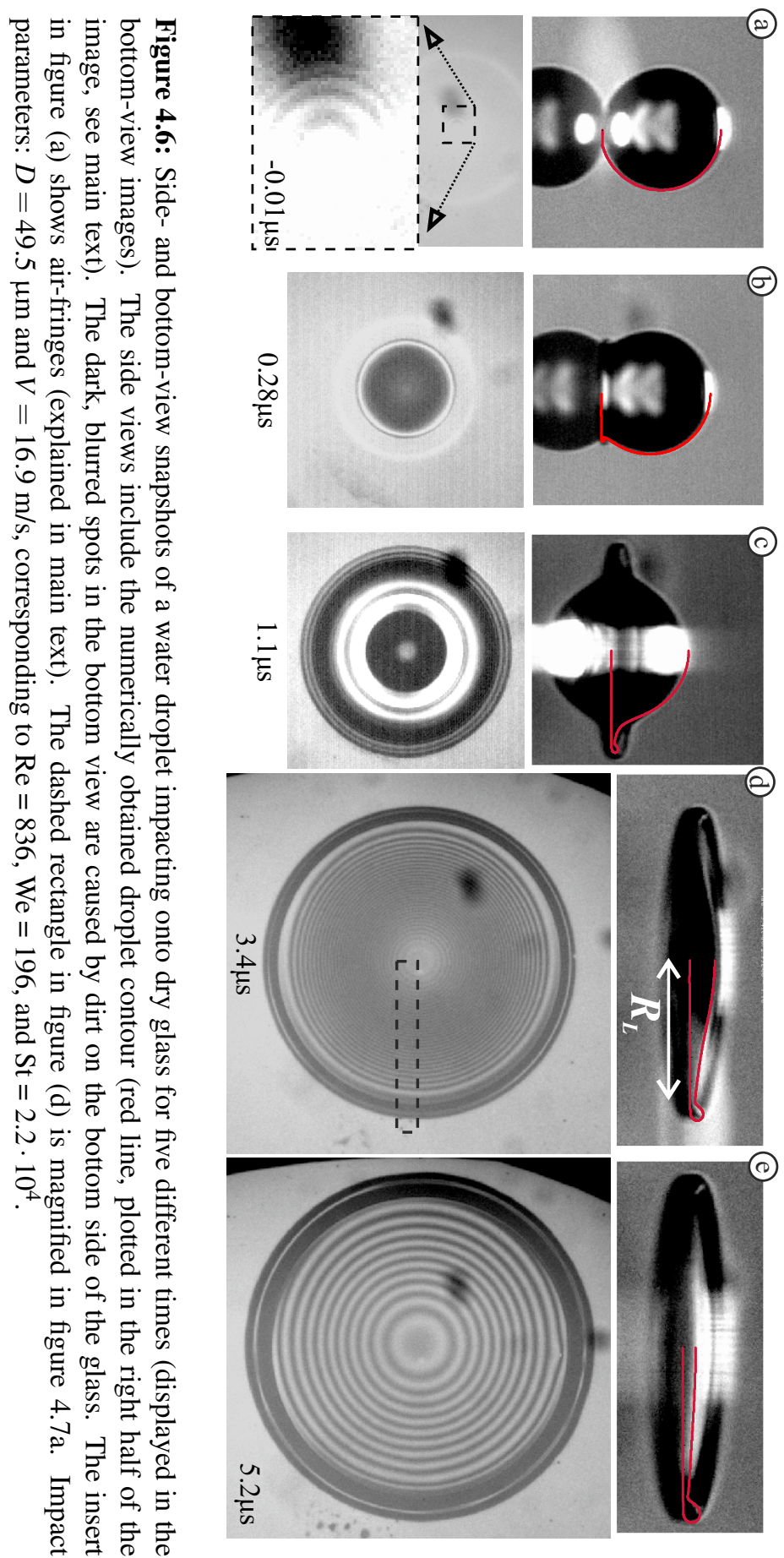
all excess volume, the profile drawn in figure $4.7 \mathrm{c}$ is obtained, with a large rim and a vanishing lamella thickness just inside the rim. Alternatively, assuming that all excess volume is present below the profile, we obtain the profile without a rim as plotted in figure $4.7 \mathrm{~d}$. The actual profile, which lies in between these two limiting cases, can be obtained with higher precision from the side view. Using the rim size given by the circle in figure 4.8a and integrating this to obtain the rim volume, both the rim radius and the film thickness are fixed. In figure $4.8 \mathrm{~b}$, the result is plotted using MATLAB (the lighting was set to make the images (a) and (b) correspond, and a mirror image of the droplet was plotted (in black) below the droplet). Finally, in figure $4.8 \mathrm{c}$, the derived profile is shown to be in good agreement with the numerical result. Theoretical profiles were derived for inviscid impact [36, 54]. However, since viscosity results in the formation of a boundary layer which affects the film thickness for impact on solid surfaces [54], these predictions deviate from our results (in particular, a much thinner lamella with a far larger radial extension is obtained from theory)and are therefore not included. As the numerical results are based on the Weber, Reynolds, and Stokes-numbers alone, the good overall agreement with the experimental droplet profiles provides first evidence for the scale-invariance of the impact events studied here. In the following sections we will discuss the different aspects of the impact in detail.

\subsubsection{Spreading dynamics}

In figure $4.9 \mathrm{a}$, the spreading dimensionless diameter $D / D_{0}$ is plotted as a function of dimensionless time $t / \tau$, for different Weber- and Reynolds numbers (indicated by symbol shape) and for impact on surfaces with different wettabilities (indicated by marker color). For $t / \tau \lesssim 3$, we found a negligible influence of the contact angle on the spreading radius, as was also observed for mm-sized droplets [55]. Good agreement between our measurements and our numerical model (solid lines) is reached . In fact, even better agreement with the numerical results is obtained than with mm-sized droplets, as in measurements with mm-sized droplets (ref. [55], plotted as *) slightly stronger spreading than in our numerical simulations is seen, for unknown reasons.

To compare our results with the literature, we require a more detailed picture of the spreading dynamics of the droplet. The impact can be divided into three different phases, of which the first two are depicted by the inset in figure 4.9b:

- The initial phase, in which the bulk of the droplet maintains its initial velocity and no lamella is ejected (blue line in the inset of figure 4.9b). The dropletsubstrate contact diameter $D_{C}$ was initially described assuming compression of the liquid in the droplet (i.e. lack of lamella ejection), resulting in $D_{C} / D_{0}=$ 


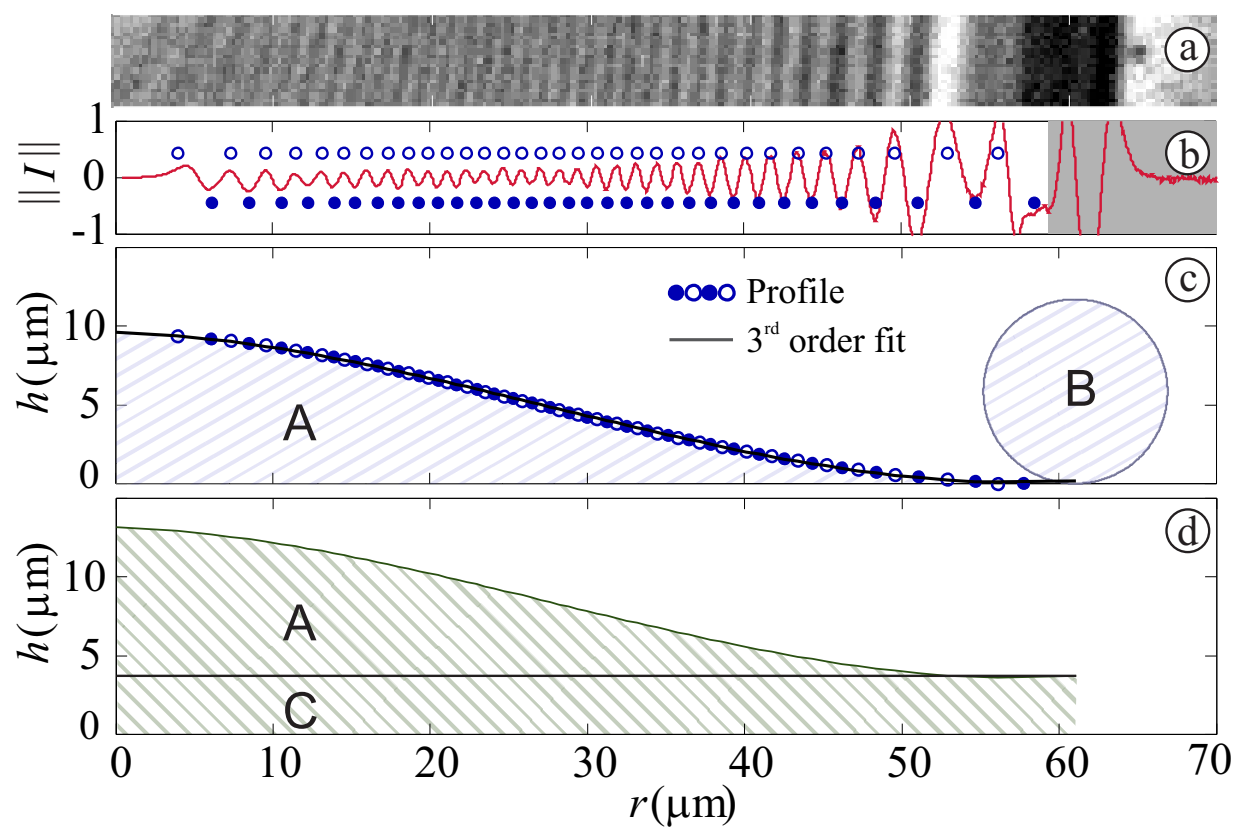

Figure 4.7: Bottom view analysis. (a) Detail of figure 4.6d, clearly showing fringes. (b) Radially-averaged intensity map of the bottom view image. The open and closed markers show the radial positions where respective bright and dark fringes were detected. The shaded area is not used for analysis: (c) The rim profile determined from the fringes is shown by the open and closed markers, corresponding to figure (b). The black line indicates a third-order fit. Integration of area A provides the volume contained under the profile, which is less than the original drop volume. Area B corresponds to the rim volume if the rim would contain all excess volume of the droplet. (d) Area C corresponds to the excess liquid if it would be fully contained under the droplet, resulting in an offset of the droplet profile equal to the height of area $\mathrm{C}$. 

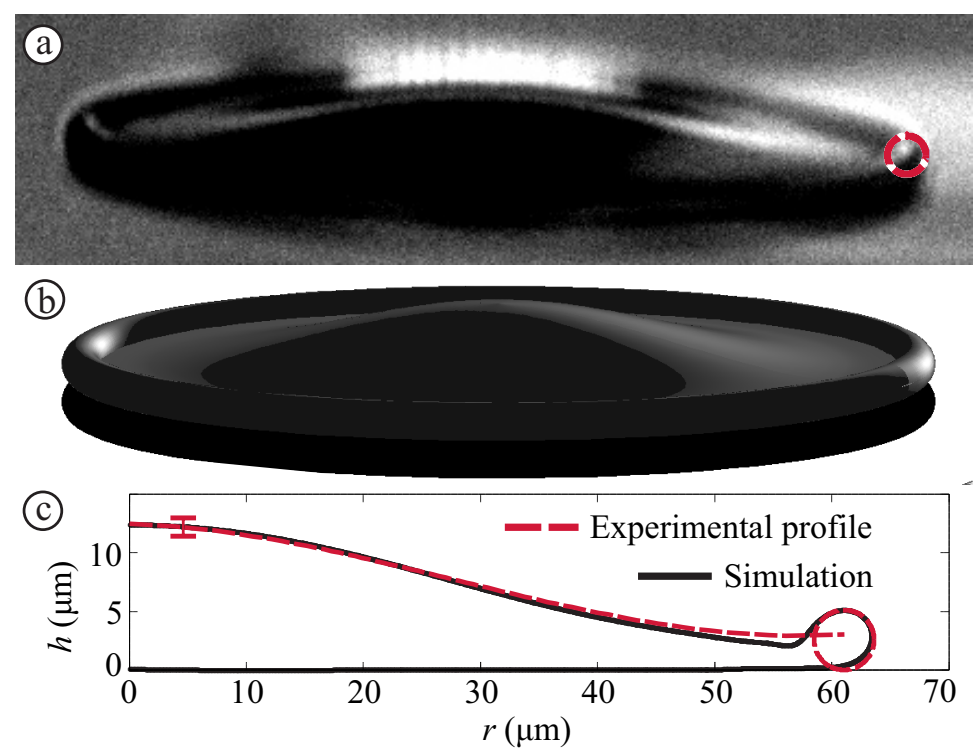

Figure 4.8: Bottom view analysis combined with side view and simulation, for the droplet analyzed in figure 4.7. (a) Side view image. In red the rim size is shown, which was used for the analysis of the bottom view images as explained in the main text. (b) Side-view image reconstructed from the fringes in the bottom-view. (c) Rim profiles determined from experiment and simulation. The red vertical line indicates the experimental error.

$2 \sqrt{t / \tau-(t / \tau)^{2}}$ from geometry arguments [55]. A modification was made by assuming incompressible flow from the droplet into a disk-shaped lamella [14, 58], but for $t / \tau \ll 1$ this result collapses to the aforementioned result from ref. [55]. Recently, the radial position of the inflection point $D_{I}$ was calculated using Wagner's theory [59], resulting in $D_{I}=\sqrt{3 t / \tau}$, and proposed as the initial wetting velocity [56]. As the transition to the next regime (where the lamella is ejected) occurs on a time scale of order $\tau / 100$, we could not yet study this regime. To conclusively measure the dynamics in this regime (also for mm-sized droplets), high-resolution measurements would be required, in particular in view of the small differences between the various predictions.

- The lamella phase. A lamella is ejected when its velocity exceeds the wetting velocity observed in the initial phase $[56,57]$. This occurs at the lamella ejection time $t_{e}$, which was recently modeled [56] by the solution of the implicit equation

$$
\sqrt{3} / 2 \operatorname{Re}^{-1}\left(t_{e} / \tau\right)^{-1 / 2}+\mathrm{We}^{-1}=C\left(t_{e} / \tau\right)^{3 / 2},
$$



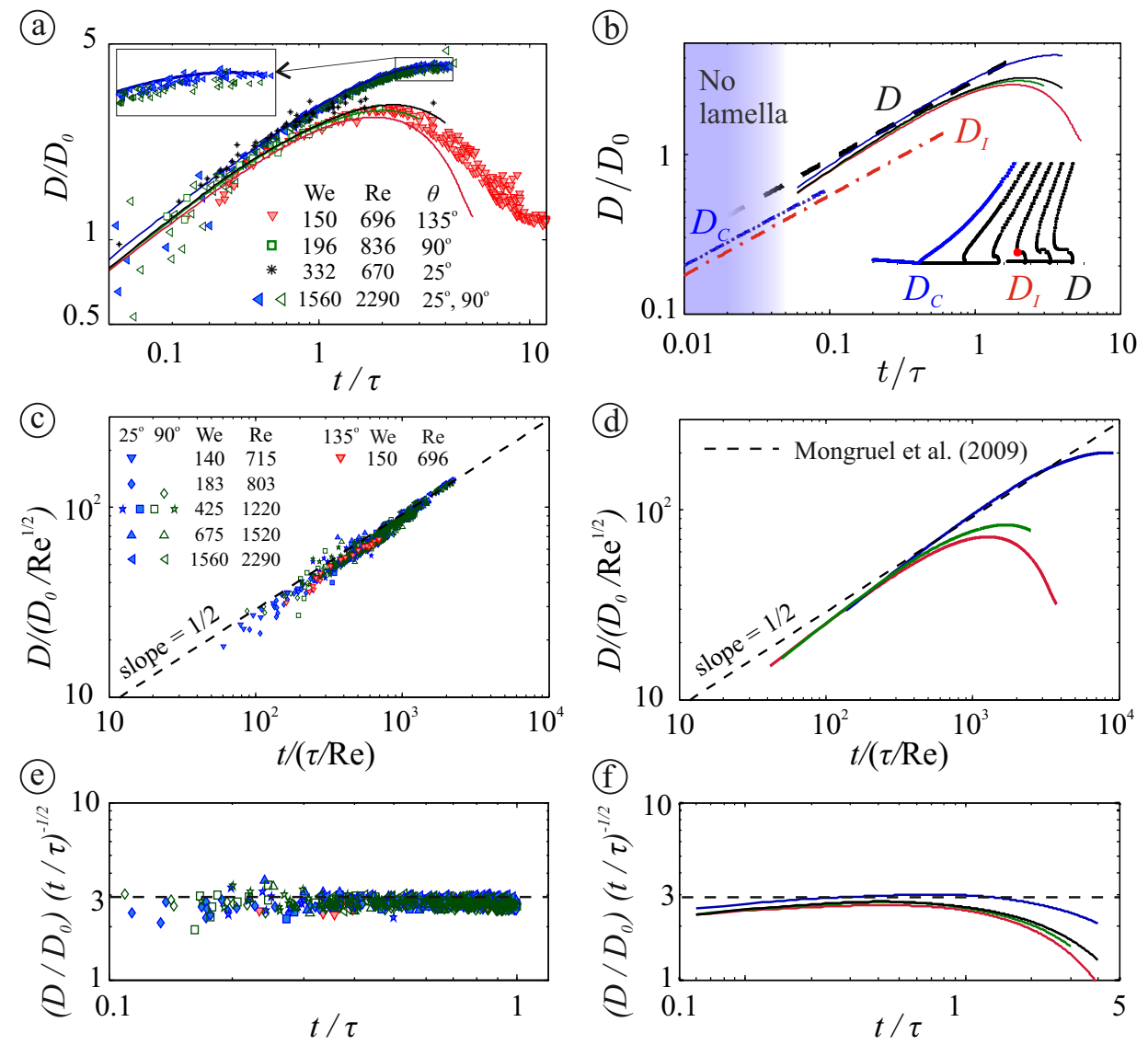

Figure 4.9: Normalized spreading diameter versus time. Left: (mainly) experiments. Right: numerical results. (a) Comparison between measurements (markers) and simulations (lines) for three different contact angles. The black stars represent mm-sized droplet data from ref. [55] $\left(D_{0}=2.7 \mathrm{~mm}\right.$ and $V_{0}=2.6 \mathrm{~m} / \mathrm{s}$, We and Re in the legend). The spreading is contactangle independent and good agreement with the numerical results is obtained, as illustrated by the collapsing curves in the inset. Final retraction of the droplet is observed only for the strongly hydrophobic surface $\left(\theta=135^{\circ}\right)$ and in the simulations $\left(\theta=180^{\circ}\right)$. (b) Development of the contact diameter before lamella ejection $D_{C}$ (blue dash-dotted line), the inflection diameter $D_{I}$ (red dashed line), and the maximal lamella diameter $D$ (black dashed line); solid lines show the numerical results corresponding to figure (a). The color-gradient indicates the predicted transition towards the lamella regime [56], as discussed in the main text. A time series of the droplet shape is shown in the right-bottom corner of the figure, illustrating the contact diameter $D_{C}$, the inflection diameter $D_{I}$, and the maximal lamella diameter $D$. (c) Normalized lamella spreading versus normalized time. The experimental data (shown for $t / \tau<1$ ) collapse to the model proposed by Mongruel et al. [57] (equation (4.2), shown as a dashed line in figures (c) to (f)) within the experimental error. (d) The numerical results (solid lines) collapse to theory [57] at intermediate times. For small times the lamella is not fully developed; in the late regime surface tension and viscosity limit the spreading. (e) \& (f): The corresponding compensated plots $\left(D / D_{0}\right)(t / \tau)^{-1 / 2}$ versus $t / \tau$ (out of which Re cancels) for figures (c) \& (d). 
with prefactor $C=1.2$. For our control parameters this yields a value of $0.02<$ $t_{e} / \tau<0.04$, illustrated by the transition from the shaded (blue) region to the white region in figure $4.9 \mathrm{~b}$. The lamella spreading velocity and radial extension were obtained by equating the inflow from the drop and the outflow into the lamella (modeled as a circular disc with an empirically obtained thickness) [57]:

$$
\frac{D_{L}(t)}{D_{0}}=2.9 \sqrt{\frac{t}{\tau}} .
$$

A comparison with our experimental results in this regime is provided in figure $4.9 \mathrm{c}$, where the axes were chosen to allow direct comparison to ref. [57]. The experimental results are limited to $4 t_{e}<t<\tau$, ensuring that only the lamella regime is captured. The model and experiments agree quantitatively within the experimental error, again evidencing scale-invariance of the drop impact dynamics. Our numerical results (figure 4.9d) show reasonable agreement for intermediate times, but deviate for small times (where the transition from the slower initial regime results in a lower value), and for large times (due to slowdown, next item).

- The slow-down phase $(t / \tau>1)$, in which surface tension and viscosity significantly slow down the spreading lamella. This eventually stops the spreading, defining the maximum spreading diameter as discussed in subsection 4.3.6.

\subsubsection{Droplet-air interaction}

As discussed in the introduction and shown in figure 4.1b, the gas in the gap between the droplet and the surface forms a dimple in the droplet and "cushions" the impact. The initial contact between the droplet and the substrate therefore occurs off-axis, and air is entrained by the dimple in the droplet. As this air cannot escape, a small bubble inside the droplet is observed.

Initially we aimed to assess the air film behavior using the fringes using bottomview interferometry, a method commonly applied to determine the air profile separating the droplet from the surface $[17,39,61]$. However, processing the fringes shown in figure 4.6a reveals a spherical droplet profile with a curvature corresponding to the droplet size, i.e. the expected dimple was not observed. A prediction of the dimple height was provided in ref. [19], yielding $H \sim 50 \mathrm{~nm}$ for our control parameters. As the resolution of the interference method is limited to $\lambda / 4=145 \mathrm{~nm}$ (with $\lambda=577 \mathrm{~nm}$ the illumination wavelength) the dimple profile cannot be resolved with bottom-view interferometry in the visible spectrum. Therefore, to assess the scale-invariance of the droplet-air interaction, we now discuss the bubble size. 


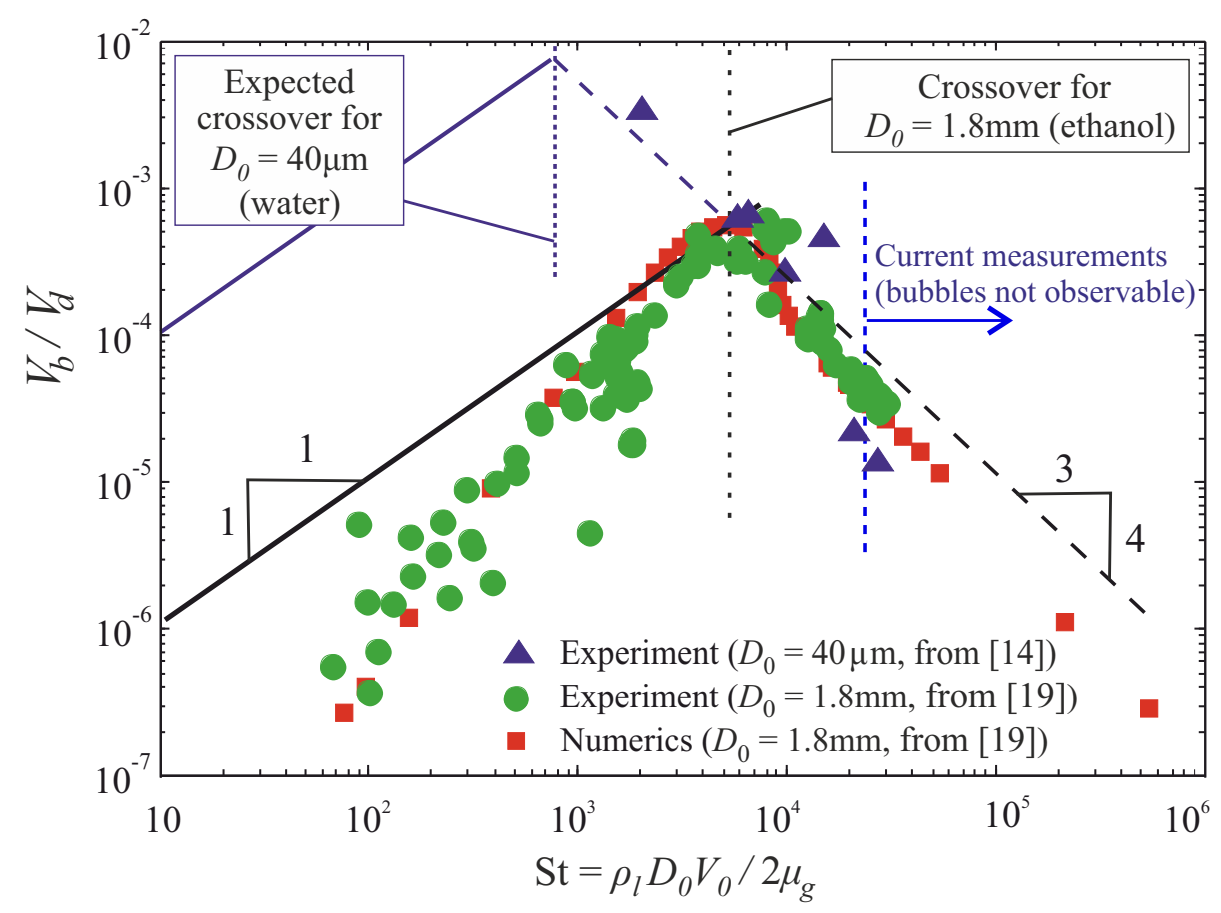

Figure 4.10: Dimensionless volume of the bubble entrained during droplet impact versus the Stokes number (St). For microdroplets, the bubble volume was measured by Van Dam and Le Clerc (2004) [14] and re-cast here (blue markers), as for the current experiments the bubble size is smaller than the optical resolution of the setup. For comparison to air entrainment by mm-sized droplets, the experimental and numerical results from Bouwhuis et al. (2012) [19] are included (green and red markers). The lines show scaling laws proposed for the capillary air entrainment regime (low-St, solid lines, from ref. [19]) and the inertial air entrainment regime (high-St, dashed lines, from refs. $[19,60])$. The vertical blue and black dashed lines indicate the crossover from the capillary to the inertial regime, for micro- and mm-sized droplet impact, respectively. 
The size of the bubble entrained by impacting droplets was determined theoretically, experimentally, and numerically for millimeter-sized droplets [19], as shown in figure 4.10. At very low Stokes numbers (i.e. slow droplets), the droplet approaches the surface so slowly that the gas can escape, and no bubble is entrained [62]. For increasing (but still low) Stokes numbers, an increasingly large bubble is observed, indicated by the solid black line in figure 4.10. However, in the high-Stokes number limit, the gas is squeezed out so strongly that again very small bubbles are observed as indicated by the black dashed line [60]. The maximum bubble size is observed at an intermediate "crossover" Stokes number, which is plotted as a vertical dotted line in figure 4.10 .

Since compressibility is likely to become important for fast micrometer-sized droplet impact $[60,63]$, the question is whether equal dimensionless droplet sizes are observed. The bubbles entrained by microdroplet impact at lower velocities than in our experiments ${ }^{\star}$ were measured by Van Dam and LeClerc [14] and are re-cast in figure 4.10. To compare these data to the model discussed, the crossover Stokes number (which depends on the Weber- and Reynolds numbers and will thus change for smaller droplets) was re-calculated for $D_{0}=40 \mu \mathrm{m}$ and plotted as a blue dotted line in figure 4.10. The data from ref. [14] collapse to the prediction, and completely lie in the high-Stokes regime. Although the scale-invariance of the low-St regime remains to be addressed, this provides strong evidence for scale-invariant entrainment of air by droplet impact in the high-St regime, i.e. that no additional dimensionless numbers (apart from $\mathrm{Re}, \mathrm{We}$, and $\mathrm{St}$ ) are required to describe the air entrainment by impacting droplets.

\subsubsection{Boundary layer development}

During impact, the radial flow over the surface results in the development of a boundary layer from the surface. Here the local boundary layer thickness $\delta_{B L}(r, t)$ is defined as the height at which $95 \%$ of the maximal horizontal velocity is reached.The red lines in figure 4.11 show the boundary layer thickness derived from the simulations, which is generally well-defined (values close to the origin $(r<5 \mu \mathrm{m})$ and early times $(t / \tau<0.06)$ are inconsistent and therefore excluded). To obtain a single boundary layer thickness value for each instant in time, $\delta(r, t)$ is spatially averaged as:

$$
\bar{\delta}_{B L}(t)=\frac{4 \int_{0}^{2 \pi} \int_{0}^{D(t) / 2} \delta_{B L}(r, t) r d r d \theta}{\pi D(t)^{2}}
$$

\footnotetext{
¥Our current experiments (at high Stokes numbers, see figure 4.10) resulted in a bubble size below the resolution of our setup.
} 

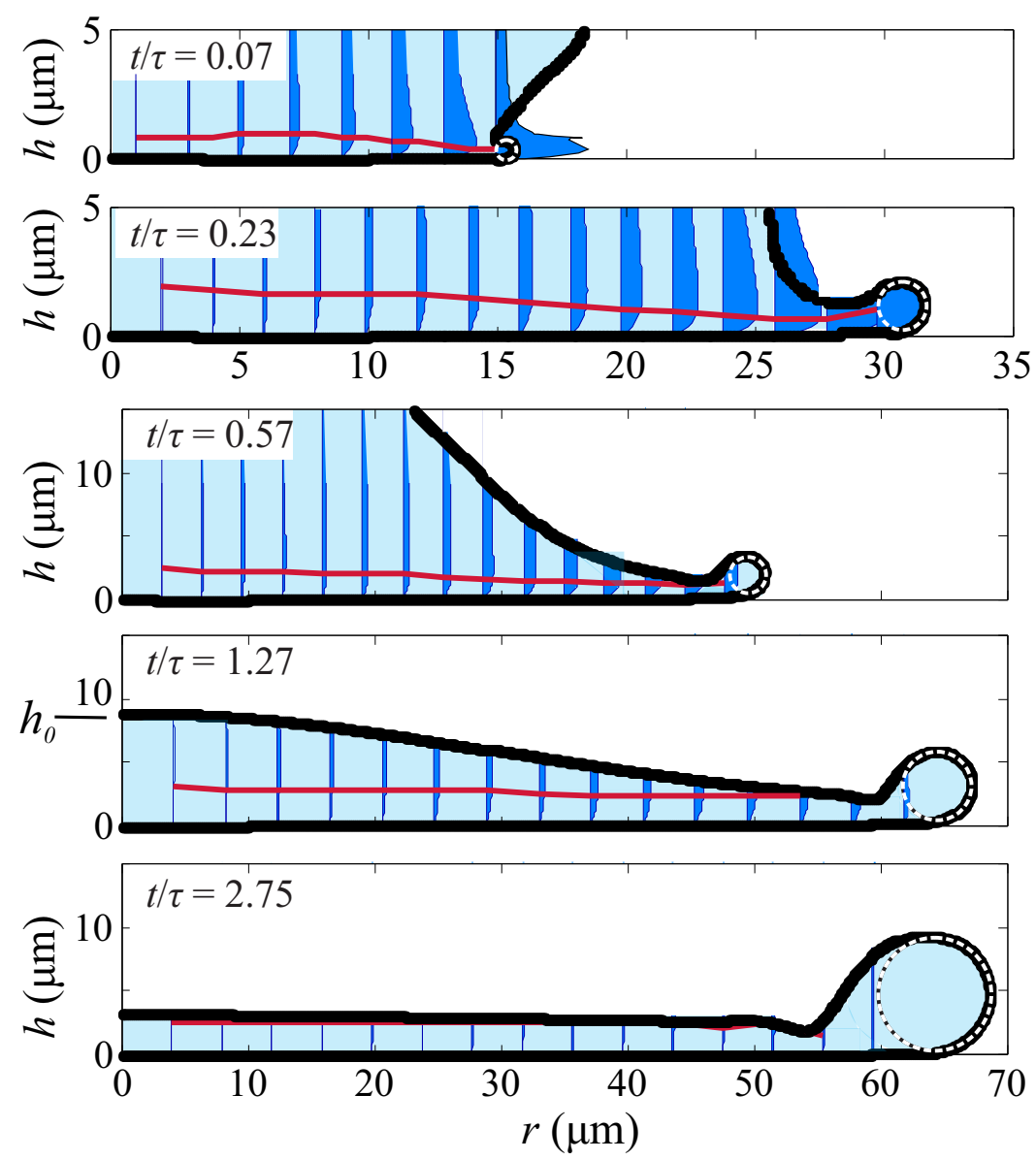

Figure 4.11: Snapshots of the simulated cross section of the droplet, for We $=196$ and $\mathrm{Re}$ $=836$. The black line indicates the droplet contour, the blue shading indicates the liquid, and the radial velocity component are plotted as filled areas (blue). The red line indicates the boundary layer $\delta(r, t)$. The white dotted lines shows the fitted rim profile. The parameter $h_{0}$ indicates the center height of the droplet, as shown left of the fourth image. Note the different scaling of the upper two images, allowing for a more detailed view. 
(a)

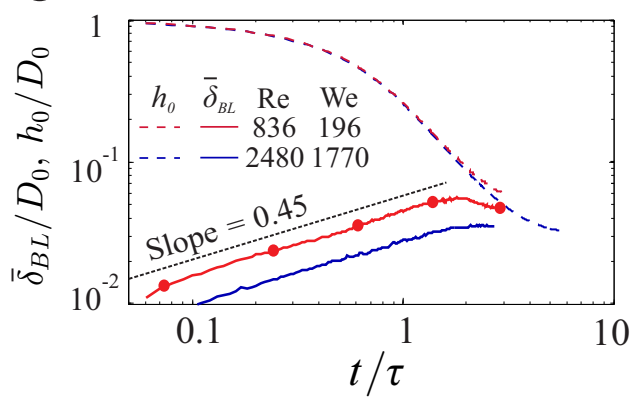

(b)

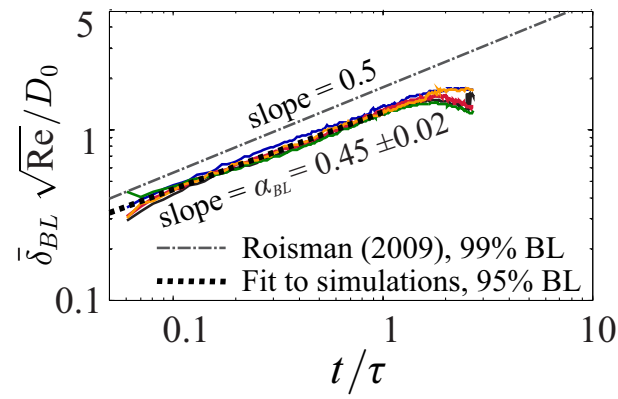

Figure 4.12: (a) Simulated space-averaged boundary layer thickness versus time for two (Re, We) pairs (in red and blue, as shown in the legend), normalized by the droplet diameter. The dashed lines indicate the center height of the droplet $h_{0}(t)$. The red markers correspond to the droplet profiles as plotted in figure 4.11. Figure (b) shows the boundary layer thicknesses normalized by $\mathrm{Re}^{-1 / 2}$ (line colors indicate Reynolds and Weber numbers as in figure 4.13 (b)), displaying a good collapse in this normalization.

with $\theta$ the angular coordinate. This spatially-averaged boundary layer thickness is plotted as a function of time in figure 4.12a. The markers on the red line correspond to the snapshots in figure 4.11. A maximum boundary layer thickness is observed at $t / \tau \approx 2.5$ when the (increasing) boundary layer thickness approaches the (decreasing) droplet thickness $h_{0}(t)$ (indicated by the dashed line in figure 4.12a). This instant is important in the determination of the maximum spreading diameter, as will be discussed in detail in section 4.3.6.

The temporal development of the boundary layer was theoretically addressed in the context of droplet impact [37]. There, an analytical flow field solution for inertiadominated inviscid flow onto a wall (long after initiation) was extended to include viscosity and a no-slip condition on the wall. This results in the development of a boundary layer with an homogeneous thickness over the full surface area (which is surprisingly consistent with figure 4.11, showing a roughly spatially homogeneous boundary layer thickness for various instants). Based on this model, a $99 \%$ boundary layer thickness as a function of time is proposed as:

$$
\delta_{99} \approx 1.88 D_{0} \sqrt{\frac{t}{\tau \mathrm{Re}}} .
$$

which is indicated by the dash-dotted line in figure $4.12 \mathrm{~b}$.

The non-dimensional boundary layer thickness obtained from our simulation is plotted in figure 4.12b. For a range of Reynolds- and Weber numbers (indicated in the caption), the results collapse to a single curve which confirms the robustness of 
(a)

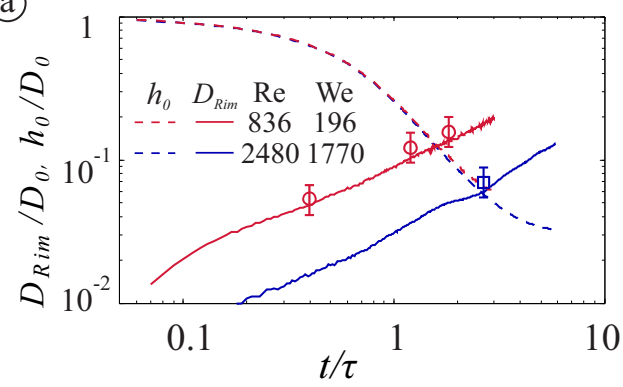

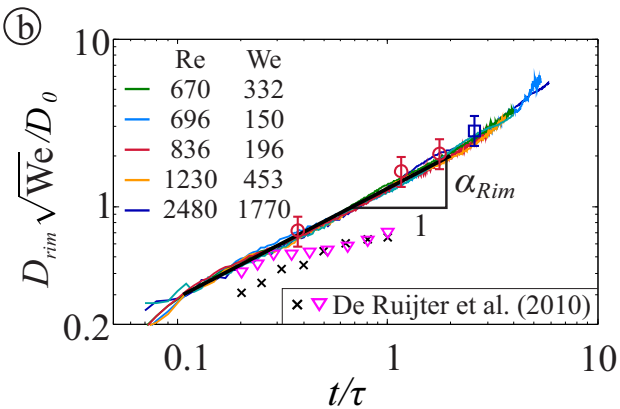

Figure 4.13: Simulated rim diameter versus time, for various (Re, We) pairs as shown in the legend. (a) Rim diameter normalized by the droplet diameter as a function of time normalized by the inertial time scale $\tau$. The markers correspond to measured rim diameters. The dashed lines indicate the center height of the droplet $h_{0}(t)$. Figure (b) shows the rim diameter normalized by $D_{0} \mathrm{We}^{-1 / 2}$. Fitting the rim evolution in time provides the exponent $\alpha_{\text {Rim }}=0.68 \pm 0.04$, which is indicated as a black line. Rim height measurements for mmsized droplets are added in figure (b), from ref. [64], for $\mathrm{We}=59$ and $\mathrm{Re}=1340$ (black crosses, $\times$ ), and $\mathrm{We}=185$ and $\mathrm{Re}=2360$ (magenta triangles $\nabla$ ).

the boundary layer scaling with respect to the Reynolds number. The curve follows $\bar{\delta}_{B L}(t) \sim D_{0} \operatorname{Re}^{-1 / 2}(t / \tau)^{\alpha_{B L}}$ with exponent $\alpha_{B L}=0.45 \pm 0.02$, and lies below the prediction of ref. [37]. This latter difference is due to the different definitions of the boundary layers: we assume a $95 \%$ boundary layer whereas a $99 \%$ boundary layer is used in ref. [37]. Also the exponent $\alpha_{B L}=0.45 \pm 0.02$ lies slightly below the theoretical exponent of $1 / 2$. This is due to the finite spreading diameter of the droplet: An instantaneous boundary layer development over the full surface is assumed in the theory [37], but the actual boundary layer development is limited to the (growing) area below the droplet. The deviations from the theoretical relationship ( $\delta \sim D_{0} \sqrt{t / \tau \mathrm{Re}}$ ) are smaller than the differences originating from different boundary layer thickness definitions. Therefore, for practical purposes, either relationship will adequately describe the boundary layer development in droplet impact.

\subsubsection{Rim development}

The formation of a pronounced rim is observed both in experiments (figure 4.6) and simulations (figure 4.11). To quantify the rim diameter, a circle is fitted to the outermost points of the simulated droplet profile as plotted in figure 4.11 (dashed white circles collapsing to the rim). The resulting rim diameter is plotted as a function of time in figure 4.13a, in which the markers show validation measurements from the side-view images which confirm the numerical results. 
In figure $4.13 \mathrm{~b}$, the rim radius is normalized by a length scale $D_{0} / \mathrm{We}^{1 / 2}$ [64]. This length scale is derived from the position which the rim has reached after a time $\tau=D_{0} / V_{0}$, assuming a typical deceleration of $V_{0}^{2} / D_{0}$ [21, 64]. Using this normalization, all our data collapse to a single curve described by $D_{\text {Rim }} / D_{0} \sim \mathrm{We}^{-1 / 2}(t / \tau)^{\alpha_{\text {Rim }}}$. Fitting our data to this equation provides $\alpha_{\text {Rim }}=0.68 \pm 0.04$. To our knowledge, this remarkably robust scaling explonent (which seems to hold also for times $t / \tau>1$ ), has not yet been theoretically explained.

To compare our results to rim size measurements of mm-sized droplets, measurements by De Ruijter et al. [64] are added to figure 4.13b (as discussed in ref. [64], earlier experimental work on the rim thickness [27, 57, 65-67] is hardly applicable to drop impact on a smooth solid surface). These measurements neither collapse on our data nor our numerical results, but they are of the same order of magnitude. The temporal evolution of the rim diameter could not be uniquely established in ref. [64] as the data set was limited to $0.2<t / \tau<1$. However, since droplet impact is generally scale-invariant, and our results partially agree to theory and experiments for mm-sized droplets [64], the herein observed scaling might also describe the rim dynamics of mm-sized droplets.

\subsubsection{Maximum spreading diameter}

The measured maximum spreading diameter $D_{\max } / D_{0}$ is plotted in figure 4.14 and compared to several theoretical $[21,26,37,44]$ and experimental results for microdroplets $[3,14,15,20,31]$. For $\mathrm{We} \approx 100$, good agreement is observed with previous experiments $[14,31]$. For larger Weber numbers, the current maximum diameter clearly lies below $[15,20]$ or above $[3,14,31]$ previous work; the differences are discussed in the appendix.

The maximum spreading diameter is the result of a competition of forces. On the one hand, inertia drives the liquid to radially expand. On the other hand, surface tension pulls back the expanding sheet, and viscosity dissipates part of the energy, limiting the maximum spreading diameter. The limiting cases, in which inertia is balanced by either surface tension or viscous dissipation (hereafter called the capillaryand viscous regime, respectively), were separately modeled by Clanet et al. [21]. The transition between these regimes is set by the parameter $P=\mathrm{We} / \mathrm{Re}^{4 / 5}=1$, as indicated by the arrow in figure 4.14 and the solid line in figure 4.2. The model shows good agreement with the current results.

However, two important additional phenomena are affecting the maximum spreading diameter [26, 36, 37, 54, 68]: (i) the formation of a rim at the edge of the expanding droplet, and (ii) viscous dissipation takes place within a thin boundary layer, as discussed. An improved approach to model spreading at We $\gg 1$ would 


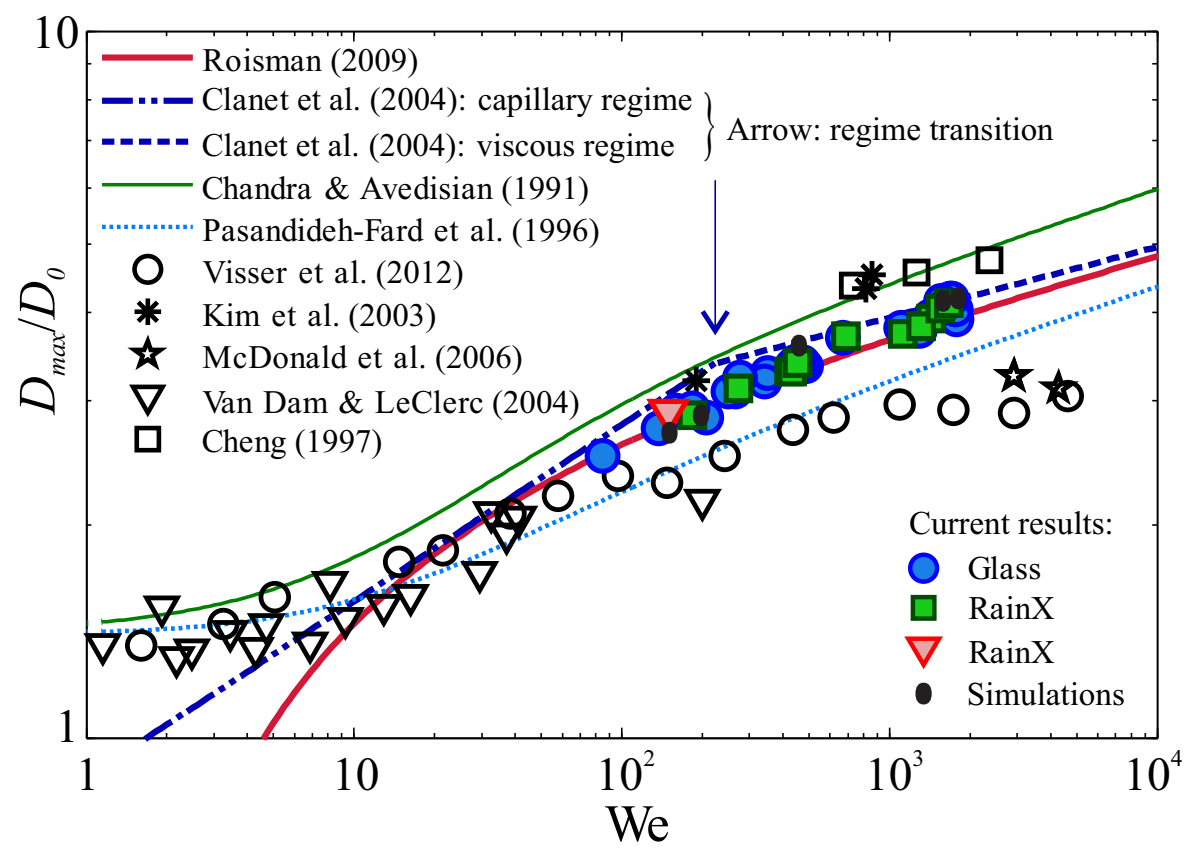

Figure 4.14: Normalized maximum spreading diameter versus the Weber number, for the current experiments (colored markers) and simulations (black dots), other micro-droplet experiments (black markers), and models (lines). The arrow indicates the transition between the capillary- and viscous-regimes proposed in ref. [21].

therefore include inertial spreading limited by boundary layer dissipation and the retraction of a rim. This approach was taken by Roisman [37], where the scaling of $D_{\max } / D_{0} \sim \mathrm{Re}^{1 / 5}$ for viscosity-limited spreading is recovered analogous to Ref. [21] (for $\mathrm{Re} \gtrsim 100$ and infinite Weber number). This result was generalized for finite Weber numbers (We $\gtrsim 10)$ by introducing a correction to account for the growth of the rim, resulting in the following semi-empirical expression for the maximal spreading diameter [37]:

$$
D_{\max } / D_{0}=0.87 \mathrm{Re}^{1 / 5}-0.40 \mathrm{Re}^{2 / 5} \mathrm{We}^{-1 / 2}
$$

Excellent agreement with our data is observed (figure 4.14). As this model was also validated for mm-sized droplets, we conclude that, for a large parameter regime, the maximum spreading diameter is captured by the Weber- and Reynolds numbers alone $e^{\S}$.

\footnotetext{
${ }^{\S}$ As discussed in ref. [26], excluding the boundary layer generally results in an over-prediction of $D_{\max }$. In this view, the excellent agreement between our measurements and the basic model from
} 


\subsubsection{Splashing}

Splashing is not observed in the current and previous [31] measurements for highspeed impact of microdroplets. Empirical splash threshold models sometimes (correctly) predict absence of a splash [69-71], but also the incidence of a splash is frequently predicted for our maximum-velocity impacts [72-74], for which $V_{0}=$ $50 \mathrm{~m} / \mathrm{s}, D_{0}=50 \mu \mathrm{m}, \operatorname{Re} \approx 2500$, and We $\approx 1770$, surface roughness $R_{a}=5 \mathrm{~nm}$, and water and air at atmospheric pressure are used.

These conflicting results clearly indicate a need for improving the physical understanding of splashing, on which great progress has recently been made. The consensus is that splashing is caused by ejection and upward deflection of the liquid lamella forming during impact $[56,75]$. In particular, two splash mechanisms have been proposed. First, the lamella may be deflected upwards by liquid touchdown on the substrate. This touchdown results in a local boundary layer, which has a "bump"shape deflecting the ejected lamella away from the surface [75]. The impact velocity threshold beyond which splashing occurs is proposed to equal the critical impact velocity for lamella ejection, provided as [75]:

$$
V_{t h}=C\left(\frac{2 \sigma^{3}}{\mu_{g}^{2} \rho D_{0}}\right)^{1 / 4} \quad, \quad 0.1<C<0.3
$$

providing $8.9<V_{t h}<27 \mathrm{~m} / \mathrm{s}$ for our system, i.e. the predicted threshold velocity is too low. However, surface roughness and air compressibility may influence the actual threshold [75]. Extrapolating figure 8 from [75] indicates that roughness on a 1 $\mathrm{nm}$-scale (which is of the order of our glass substrate roughness) is expected to further decrease the splash threshold impact velocity. Another factor which potentially suppresses splashing for the current small-scale droplets is that the touchdown occurs in a different way as compared to the model in [75], since the air film thickness is expected to become thinner than $\sim 10$ times the mean free path length of the gas molecules. In this regime the droplet's touchdown is highly nontrivial. Although the subtleties for this case have been discussed in detail for mm-sized droplets [75], they may be different for the current regime. Extending this model to fast microdroplet impact would provide an opportunity for further validation.

A second proposed splash threshold is based on a competition between destabilization of the lamella by the air film under the droplet, and the stabilizing effect of surface tension [32]. This model (which is based on dimensional analysis, and

ref. [21] is surprising. However, as the spreading stops when the boundary layer equals the lamella thickness, assuming the lamella thickness as the relevant viscous length scale (instead of explicitly incorporating the boundary layer) results in a very minor error. 
therefore does not explicitly include any physical mechanisms) predicts splashing for

$$
V_{0} \gtrsim \frac{\rho \sigma^{2} k_{B} T}{\gamma \mu M_{G} P^{2} D_{0}}
$$

with $k_{B}$ Boltzmann's constant, $T$ the temperature, $M_{G}$ the molecular weight of the gas, $\gamma$ the adiabatic constant of the gas, and $P$ the pressure of the ambient air. For our $50 \mu \mathrm{m}$-droplets in air, this yields a splashing threshold velocity of $\sim 500 \mathrm{~m} / \mathrm{s}$, thus no splashing is predicted for our droplets with impact velocities up to $50 \mathrm{~m} / \mathrm{s}$.

Recently, a similar approach has been translated into a complete physical model. Here, the lift-off of the lamella is assumed to be governed by the air surrounding the droplet (by both air compression below the lamella and a lift force due to a lowpressure area above the lamella) [56]. Another key ingredient is the (growing) rim diameter. If the lamella is lifted faster than the rim grows, the rim will not touch the substrate and splashing is predicted. However, if the rim diameter increases faster than the lamella lifts away from the surface, the rim will touch the substrate and splashing is suppressed. This model successfully predicts the splash threshold of $\mathrm{mm}$-sized droplets, for a variety of liquids. However, a key model assumption is that the air film thickness exceeds the mean free path length of the gas molecules. As this condition is violated here, a prediction cannot be obtained for our control parameters.

As yet, our observation of non-splashing impact is predicted only by empirical models $[32,69,70]$. As the model by Xu et al. [32] model contains most physical insights and has been successfully extended to explain the splash threshold droplets impacting on hot surfaces [29], it seems to be the best splash prediction model as yet. However, since only scaling arguments are used, details of the physics of splashing remain to be understood. Recent attempts provide validated splash threshold predictions for mm-sized droplets [56,75], but neither of these approaches is applicable to the micrometer domain since the continuum assumption of the gas breaks down. As the underlying physics of these models are fundamentally different, details of the splash mechanism remain to be fully understood and future modeling efforts are expected. The current results, in which no splash is observed despite high impact velocities, may provide a powerful tool for validating these models.

\subsection{Conclusions}

In this work the similarities and differences between mm-sized droplet impact (for which great progress has been made recently) and micrometer-sized droplet impact (which is key for industrial applications but has hardly been addressed) are studied in detail. Highly reproducible micrometer-scale droplets were generated and visualized 
using flash-illumination. Side-view images show the spreading droplet at a temporal resolution exceeding $10^{7}$ frames per second. Bottom-view images reveal a fringe pattern due to reflection of the (flat) glass interface and the (curved) top surface of the droplet, and were used to resolve the droplet shape at sub-micron resolution. A numerical model is developed and extensively validated using the side-and bottomview measurements. The model is used to study the boundary layer development and the size of the rim, which are challenging to quantify experimentally.

The spreading dynamics, droplet-air interaction, boundary layer development, rim development, maximum droplet spreading diameter, and splashing threshold are investigated in detail. The main conclusions are:

1. The spreading dynamics can be divided into different phases. The initial phase, prior to the ejection of a lamella, could not be investigated due to the extremely short time scales involved. In the lamella phase, a scaling $D / D_{0} \sim \sqrt{t / \tau}$ is observed, which is in quantitative agreement with ref. [57]. The final (slowdown) phase results in the maximal spreading diameter, see item 5.

2. The air bubble size entrained during microdroplet impact decreases with increasing impact velocities (for the currently studied impact velocities $V_{0}>$ $10 \mathrm{~m} / \mathrm{s}$ ). The scaling of the air bubble size collapses to results for mm-sized droplet impact in the high-Stokes number regime. The threshold Stokes number separating the high- and low Stokes number regimes is different, which is expected since this parameter is size-dependent.

3. The boundary layer thickness scales as $\bar{\delta}_{B L}(t) \sim D_{0} \mathrm{Re}^{-1 / 2}(t / \tau)^{\alpha}$ with an effective exponent $\alpha_{B L}=0.45 \pm 0.02$. The Reynolds number dependence follows the expected scaling of $\bar{\delta}_{B L}(t) \sim D_{0} \operatorname{Re}^{-1 / 2} \sqrt{t / \tau}$, but the temporal progression is somewhat lower. In addition, details of the definition of the boundary layer have a pronounced effect, which is expected to influence variables which depend on the boundary-layer thickness.

4. The rim diameter follows a scaling $D_{\text {Rim }} / D_{0} \sim \mathrm{We}^{-1 / 2}(t / \tau)^{\alpha_{\text {Rim }}}$, with effective exponent $\alpha_{\text {Rim }}=0.68 \pm 0.04$. To our knowledge, this scaling is observed for the first time. The temporal exponent and the remarkable robustness of this scaling for times $t / \tau>1$ remain to be explained.

5. The maximal droplet spreading diameter is robustly captured by models balancing the inertia of the droplet to viscous dissipation in the boundary layer [37]. 
6. Despite our high impact velocities up to $V_{0}=50 \mathrm{~m} / \mathrm{s}$, no splash is observed here. Only the model by Xu et al. [32] correctly predicts this outcome. This model is based on scaling arguments, and does not contain detailed (force) balances and prefactors. Alternative models capture the physics of splashing in more detail [56, 75], but since the continuum assumption is violated for the thin air film between the droplet and the substrate these models cannot be applied here.

As far as could be observed, droplet impact is scale-invariant, i.e. mm-sized droplet experiments have a predictive value as long as the same Reynolds, Weber, and Stokes number are used. Therefore, the vast progress in understanding $\mathrm{mm}$-sized droplet impact can be applied to optimize a wide range of applications in which fast, $\mu \mathrm{m}$-sized droplet impact on a dry surface is a key process step, such as (3D)(bio)printing, thermal spraying, spray cleaning, and laser-induced forward transfer. Future studies may address additional phenomena including lamella break-up [76], the influence of the slip length $[54,76]$, and droplet compressibility $[76,77]$ as these are highly relevant for impact of even faster and smaller droplets [76].

\section{Appendix: Experimental results in drop spreading}

Figure 4.14 shows the normalized maximum spreading diameter. For We $>100$, the current maximum diameter clearly lies below $[15,20]$ or above $[3,14,31]$ previous work; the discrepancies are discussed here.

The results exceeding our results were taken for droplets with $D_{0}=235 \pm 30 \mu \mathrm{m}$ [15] and $D_{0}=230 \mu \mathrm{m}$ [20], i.e. larger than our droplets with $D_{0}=48 \pm 3 \mu \mathrm{m}$. As the spreading depends on both We and $\operatorname{Re}$ (and not on We alone as displayed in the graph), a larger spreading is expected. Assuming $\mathrm{We}=800$ and droplet diameters $D_{0}=[48,235] \mu \mathrm{m}$, we obtain $\mathrm{Re}=[1661,3666]$, respectively. Using the model of Roisman [37], a 15\% larger spreading diameter is expected for the larger $\left(D_{0}=\right.$ $230 \mu \mathrm{m})$ droplets. The data around $\mathrm{We}=800$ shows a $20 \%$ increase, i.e. a slightly larger but similar value.

Our previous results [31] are clearly lower than the current results (at $\mathrm{We}=800$, a $21 \%$ decrease is found). This is due to three reasons. First, the high-Weber number measurements were taken for smaller droplets $(\sim 20 \mu \mathrm{m})$, as the previous droplet generation method did not produce consistent droplet sizes for all velocities (ref. [31], figure 2c). An analysis similar to the previous paragraphs shows an expected decrease in spreading diameter of 5\%. Second, the current measurements show that the maximum spreading is reached by the cylindrical rim, i.e. above the surface. Just after 
reaching its maximum spreading the rim retracts around $3 \%$, while (for hydrophilic surfaces) the contact line initially sticks to the surface. The temporal resolution of the previous measurements $(1 \mu \mathrm{s})$ did not allow to visualize this retraction, and thus in the previous measurement the maximum wetted diameter was measured instead of the maximum extension of the rim. This also accounts for a part of the discrepancy. Finally, the spatial resolution of the previous experiments was much more limited (ref. [31], figure 3d). We appear to have over-estimated the measurement accuracy at the droplet edge, and we conclude that high-resolution measurements are required for adequate spreading measurements. As this problem worsens with increasing velocities, the plateau of $D_{\max } / D_{0}$ reported for $\mathrm{We}>1000[20,31]$ requires validation.

Then, the maximum spreading of hot metal droplets onto a glass surface (ref. [3], figure 3b) also lies below current results. As the glass temperature was much below the metal melting temperature, solidification might have limited the spreading diameter. In addition, the surface tension of a hot $(\sim 3300 \mathrm{~K})$ droplet while cooling down and oxidizing is hard to establish, i.e. the Weber number is poorly defined. Here we take a value of $\sigma=2.3 \mathrm{~N} / \mathrm{m}$ from ref. [78] and estimate $\sigma=2.0 \mathrm{~N} / \mathrm{m}$ for the steelchromium alloy reported [79], as values for the actual alloy may not exist. Finally, the initial droplet diameter had a high uncertainty (Particles with $D_{0}=60 \pm 38 \mu \mathrm{m}$ with an average of $D_{0}=40 \mu \mathrm{m}$ were reported). Although a 40 -measurement average was taken, this could strongly add to the error. Therefore, in view of these major uncertainties, we are surprised by the reasonable agreement with our measurements.

Finally, one data point from previous high-quality measurements [14] lies significantly below our data. As this single data point also lies below the curve plotted in ref. [14], we took a detailed look at the experimental parameters. The authors report a maximum impact velocity of $V_{0}=13.8 \mathrm{~m} / \mathrm{s}$ and a maximum diameter of $D_{0}=42 \mu \mathrm{m}$, corresponding to $\mathrm{We}=111$ for water. However, the data point was plotted at $\mathrm{We} \approx 200$, resulting in a minor discrepancy. In conclusion, we think the current results are at least as reliable as (and mostly consistent with) previous work.

\section{References}

[1] S. W. Park and C. S. Lee, Macroscopic and microscopic characteristics of a fuel spray impinged on the wall, Experiments in Fluids 37, 745 (2004).

[2] A. Moreira, A. Moita, and M. Panão, Advances and challenges in explaining fuel spray impingement: How much of single droplet impact research is useful?, Progress in Energy and Combustion Science 36, 554 (2010).

[3] A. McDonald, M. Lamontagne, C. Moreau, and S. Chandra, Impact of plasma-sprayed metal particles on hot and cold glass surfaces, Thin Solid Films 514, 212 (2006).

[4] H. Troesch, Die Zerstäubung von Flüssigkeiten, Chemie Ingenieur Technik (1954). 
[5] D. Attinger, Z. Zhao, and D. Poulikakos, An Experimental Study of Molten Microdroplet Surface Deposition and Solidification: Transient Behavior and Wetting Angle Dynamics, Journal of Heat Transfer 122, 544 (2000).

[6] S. Tasoglu and U. Demirci, Bioprinting for stem cell research., Trends in Biotechnology 31, 10 (2013).

[7] T. Boland, X. Tao, B. J. Damon, B. Manley, P. Kesari, S. Jalota, and S. Bhaduri, Drop-on-demand printing of cells and materials for designer tissue constructs, Materials Science and Engineering: C 27, 372 (2007).

[8] B. Guillotin, A. Souquet, S. Catros, M. Duocastella, B. Pippenger, S. Bellance, R. Bareille, M. Rémy, L. Bordenave, J. Amédée, and F. Guillemot, Laser assisted bioprinting of engineered tissue with high cell density and microscale organization., Biomaterials 31, 7250 (2010).

[9] A. Klini, P. A. Loukakos, D. Gray, A. Manousaki, and C. Fotakis, Laser induced forward transfer of metals by temporally shaped femtosecond laser pulses., Optics Express 16, 11300 (2008).

[10] J. Schlabe, C. Johnen, R. Schwartlander, V. Moser, B. Hartmann, J. C. Gerlach, and M. V. Küntscher, Isolation and culture of different epidermal and dermal cell types from human scalp suitable for the development of a therapeutical cell spray., Burns : Journal of the International Society for Burn Injuries 34, 376 (2008).

[11] H. F. Okorn-Schmidt, F. Holsteyns, a. Lippert, D. Mui, M. Kawaguchi, C. Lechner, P. E. Frommhold, T. Nowak, F. Reuter, M. B. Pique, C. Cairos, and R. Mettin, Particle Cleaning Technologies to Meet Advanced Semiconductor Device Process Requirements, ECS Journal of Solid State Science and Technology 3, N3069 (2013).

[12] W. Melfo, H. Bolt, M. Rijnders, D. Staalman, C. B. Castro, D. Crowther, and B. Jana, Experimental Study on Primary Scale Formation and Descalability on Steels Containing $\mathrm{Ni}$ and $\mathrm{Ni}+\mathrm{Si}$, ISIJ International 53, 866 (2013).

[13] M. Versluis, High-speed imaging in fluids, Experiments in Fluids 54, 1458 (2013).

[14] D. B. van Dam and C. Le Clerc, Experimental study of the impact of an ink-jet printed droplet on a solid substrate, Physics of Fluids 16, 3403 (2004).

[15] H.-Y. Kim, S.-Y. Park, and K. Min, Imaging the high-speed impact of microdrop on solid surface, Review of Scientific Instruments 74, 4930 (2003).

[16] S. Jung and I. M. Hutchings, The impact and spreading of a small liquid drop on a non-porous substrate over an extended time scale, Soft Matter 8, 2686 (2012).

[17] R. C. A. van der Veen, T. Tran, D. Lohse, and C. Sun, Direct measurements of air layer profiles under impacting droplets using high-speed color interferometry, Physical Review E 85, 026315 (2012).

[18] R. Bhola and S. Chandra, Parameters controlling solidification of molten wax droplets falling on a solid surface, Journal of Materials Science 4, 4883 (1999).

[19] W. Bouwhuis, R. C. A. van der Veen, T. Tran, D. L. Keij, K. G. Winkels, I. R. Peters, D. van der Meer, C. Sun, J. H. Snoeijer, and D. Lohse, Maximal Air Bubble Entrainment at Liquid-Drop Impact, Physical Review Letters 109, 264501 (2012).

[20] L. Cheng, Dynamic spreading of drops impacting onto a solid surface, Industrial \& Engineering Chemistry Process Design Development 16, 192 (1977).

[21] C. Clanet, C. Béguin, D. Richard, and D. Quéré, Maximal deformation of an impacting drop, Journal of Fluid Mechanics 517, 199 (2004).

[22] R. Ford and C. Furmidge, Impact and spreading of spray drops on foliar surfaces, Soc Chem Ind Monogr 417 (1967). 
[23] H. Fukanuma and A. Ohmori, in Proc. Natl. Therm. Spray Conf. (ASM International, Boston, MA, 1994), pp. 563-568.

[24] G. Lagubeau, M. A. Fontelos, C. Josserand, A. Maurel, V. Pagneux, and P. Petitjeans, Spreading dynamics of drop impacts, Journal of Fluid Mechanics 713, 50 (2012).

[25] H. Marmanis and S. T. Thoroddsen, Scaling of the fingering pattern of an impacting drop, Physics of Fluids 8, 1344 (1996).

[26] M. Pasandideh-Fard, Y. M. Qiao, S. Chandra, and J. Mostaghimi, Capillary effects during droplet impact on a solid surface, Physics of Fluids 8, 650 (1996).

[27] C. D. Stow and M. G. Hadfield, An Experimental Investigation of Fluid Flow Resulting from the Impact of a Water Drop with an Unyielding Dry Surface, Proceedings of the Royal Society A: Mathematical, Physical and Engineering Sciences 373, 419 (1981).

[28] S. Thoroddsen and J. Sakakibara, Evolution of the fingering pattern of an impacting drop, Physics of Fluids 10, 1359 (1998).

[29] T. Tran, H. J. J. Staat, A. Prosperetti, C. Sun, and D. Lohse, Drop Impact on Superheated Surfaces, Physical Review Letters 108, 036101 (2012).

[30] P. Tsai, R. C. A. van der Veen, M. van de Raa, and D. Lohse, How micropatterns and air pressure affect splashing on surfaces., Langmuir 26, 16090 (2010).

[31] C. W. Visser, Y. Tagawa, C. Sun, and D. Lohse, Microdroplet impact at very high velocity, Soft Matter 8, 10732 (2012).

[32] L. Xu, W. Zhang, and S. Nagel, Drop splashing on a dry smooth surface, Physical Review Letters 94, 184505 (2005).

[33] L. Xu, L. Barcos, and S. Nagel, Splashing of liquids: Interplay of surface roughness with surrounding gas, Physical Review E 76, 066311 (2007).

[34] M. Rein, Phenomena of liquid drop impact on solid and liquid surfaces, Fluid Dynamics Research 12, 61 (1993).

[35] A. Yarin, Drop impact dynamics: splashing, spreading, receding, bouncing, Annu. Rev. Fluid Mech. 38, 159 (2006).

[36] J. Eggers, M. a. Fontelos, C. Josserand, and S. Zaleski, Drop dynamics after impact on a solid wall: Theory and simulations, Physics of Fluids 22, 062101 (2010).

[37] I. V. Roisman, Inertia dominated drop collisions. II. An analytical solution of the NavierStokes equations for a spreading viscous film, Physics of Fluids 21, 052104 (2009).

[38] J. M. Kolinski, S. M. Rubinstein, S. Mandre, M. P. Brenner, D. A. Weitz, and L. Mahadevan, Skating on a Film of Air: Drops Impacting on a Surface, Physical Review Letters 108, 074503 (2012).

[39] M. M. Driscoll and S. R. Nagel, Ultrafast Interference Imaging of Air in Splashing Dynamics, Physical Review Letters 107, 154502 (2011).

[40] S. Mandre, M. Mani, and M. P. Brenner, Precursors to splashing of liquid droplets on a solid surface., Physical Review Letters 102, 134502 (2009).

[41] M. Mani, S. Mandre, and M. Brenner, Events before droplet splashing on a solid surface, Journal of Fluid Mechanics 647, 163 (2010).

[42] S. T. Thoroddsen, T. G. Etoh, K. Takehara, N. Ootsuka, and Y. Hatsuki, The air bubble entrapped under a drop impacting on a solid surface, Journal of Fluid Mechanics 545, 203 (2005).

[43] J. S. Lee, B. M. Weon, J. H. Je, and K. Fezzaa, How Does an Air Film Evolve into a Bubble During Drop Impact?, Physical Review Letters 109, 204501 (2012). 
[44] S. Chandra and C. T. Avedisian, On the Collision of a Droplet with a Solid Surface, Proceedings of the Royal Society A: Mathematical, Physical and Engineering Sciences 432, 13 (1991).

[45] V. Mehdi-Nejad, J. Mostaghimi, and S. Chandra, Air bubble entrapment under an impacting droplet, Physics of Fluids 15, 173 (2003).

[46] S. D. Aziz and S. Chandra, Impact, recoil and splashing of molten metal droplets, International Journal of Heat and Mass Transfer 43, 2841 (2000).

[47] D. Richard, C. Clanet, and D. Quéré, Contact time of a bouncing drop, Nature 417, 811 (2002).

[48] M. Reyssat, D. Richard, C. Clanet, and D. Quéré, Dynamical superhydrophobicity, Faraday Discussions 146, 19 (2010).

[49] A. Checco, P. Guenoun, and J. Daillant, Nonlinear Dependence of the Contact Angle of Nanodroplets on Contact Line Curvature, Physical Review Letters 91, 186101 (2003).

[50] N. R. Lindblad and J. M. Schneider, Production of uniform-sized liquid droplets, Journal of Scientific Instruments 42, 635 (1965).

[51] P. E. Frommhold, A. Lippert, F. L. Holsteyns, and R. Mettin, High-speed monodisperse droplet generation by ultrasonically controlled micro-jet breakup, Experiments in Fluids 55, 1716 (2014).

[52] P. Kim, M. J. Kreder, J. Alvarenga, and J. Aizenberg, Hierarchical or not? Effect of the length scale and hierarchy of the surface roughness on omniphobicity of lubricant-infused substrates, Nano Letters 13, 1793 (2013).

[53] S. Popinet, An accurate adaptive solver for surface-tension-driven interfacial flows, Journal of Computational Physics 228, 5838 (2009).

[54] H. Lastakowski, F. Boyer, a. L. Biance, C. Pirat, and C. Ybert, Bridging local to global dynamics of drop impact onto solid substrates, Journal of Fluid Mechanics 747, 103 (2014).

[55] R. Rioboo, M. Marengo, and C. Tropea, Time evolution of liquid drop impact onto solid, dry surfaces, Experiments in Fluids 33, 112 (2002).

[56] G. Riboux and J. Gordillo, The critical impact speed for the splash of a drop, arXiv preprint arXiv:1401.6943 1 (2014).

[57] A. Mongruel, V. Daru, F. Feuillebois, and S. Tabakova, Early post-impact time dynamics of viscous drops onto a solid dry surface, Physics of Fluids 21, 032101 (2009).

[58] H.-Y. Kim, Z. C. Feng, and J.-H. Chun, Instability of a liquid jet emerging from a droplet upon collision with a solid surface, Physics of Fluids 12, 531 (2000).

[59] H. Wagner, Phenomena associated with impacts and sliding on liquid surfaces, Z. Angew. Math. Mech 4, (1932).

[60] P. D. Hicks and R. Purvis, Air cushioning in droplet impacts with liquid layers and other droplets, Physics of Fluids 23, 062104 (2011).

[61] J. M. Kolinski, L. Mahadevan, and S. M. Rubinstein, Lift-Off Instability During the Impact of a Drop on a Solid Surface, Physical Review Letters 112, 134501 (2014).

[62] A. Eddi, K. G. Winkels, and J. H. Snoeijer, Short time dynamics of viscous drop spreading, Physics of Fluids 25, 013102 (2013).

[63] P. D. Hicks and R. Purvis, Liquidsolid impacts with compressible gas cushioning, Journal of Fluid Mechanics 735, 120 (2013).

[64] J. de Ruiter, R. E. Pepper, and H. A. Stone, Thickness of the rim of an expanding lamella near the splash threshold, Physics of Fluids 22, 022104 (2010).

[65] D. C. D. Roux and J. J. Cooper-White, Dynamics of water spreading on a glass surface., Journal of colloid and interface science 277, 424 (2004). 
[66] I. V. Roisman, K. Horvat, and C. Tropea, Spray impact: Rim transverse instability initiating fingering and splash, and description of a secondary spray, Physics of Fluids 18, 102104 (2006).

[67] E. Villermaux and B. Bossa, Drop fragmentation on impact, Journal of Fluid Mechanics 668, 412 (2011).

[68] S. Tabakova, F. Feuillebois, A. Mongruel, V. Daru, and S. Radev, First stages of drop impact on a dry surface: asymptotic model, Zeitschrift für angewandte Mathematik und Physik 63, 313 (2012).

[69] R. L. V. Wal, G. M. Berger, and S. D. Mozes, The splash/non-splash boundary upon a dry surface and thin fluid film, Experiments in Fluids 40, 53 (2005).

[70] C. S. Stevens, Scaling of the splash threshold for low-viscosity fluids, EPL (Europhysics Letters) 106, 24001 (2014).

[71] K.-L. Pan, K.-C. Tseng, and C.-H. Wang, Breakup of a droplet at high velocity impacting a solid surface, Experiments in Fluids 48, 143 (2009).

[72] C. Mundo, M. Sommerfeld, and C. Tropea, Droplet-wall collisions: Experimental studies of the deformation and breakup process, International Journal of Multiphase Flow 21, 151 (1995).

[73] K. Range and F. Feuillebois, Influence of surface roughness on liquid drop impact, Journal of Colloid and Interface Science 30, 16 (1998).

[74] G. E. Cossali, a. Coghe, and M. Marengo, The impact of a single drop on a wetted solid surface, Experiments in Fluids 22, 463 (1997).

[75] S. Mandre and M. Brenner, The mechanism of a splash on a dry solid surface, Journal of Fluid Mechanics 690, 148 (2012).

[76] R. Zhang, S. Farokhirad, T. Lee, and J. Koplik, Multiscale liquid drop impact on wettable and textured surfaces, Physics of Fluids 26, 082003 (2014).

[77] J. Field, J. Dear, and J. Ogren, The effects of target compliance on liquid drop impact, Journal of Applied Physics 65, 533 (1989).

[78] W. M. Haynes, D. R. Lide, and T. J. Bruno, CRC Handbook of Chemistry and Physics (CRC Press, Boca Raton, Fl, USA, 2012).

[79] K. Mukai, L. Zushu, and M. Zeze, Surface tension and wettability of liquid Fe-16 mass \% Cr-O alloy with alumina, Materials Transactions 43, 1724 (2002). 


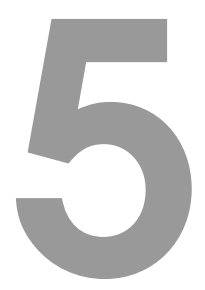

\section{Ejection regimes in picosecond laser-induced forward transfer of metals * $\dagger$}

Laser-induced forward transfer (LIFT) is a 3D direct-write method suitable for precision printing of various materials including pure metals. To understand the ejection mechanism and thereby improve deposition, here we present visualizations of ejection events at high spatial (sub- $\mu \mathrm{m}$ ) and temporal resolutions, for picosecond LIFT of copper and gold films with a thickness $50 \mathrm{~nm} \leq d \leq 400 \mathrm{~nm}$. For increasing fluences, this reveals the fluence threshold below which no ejection is observed, followed by the release of a metal cap (i.e. a hemisphereshaped droplet), the formation of an elongated jet, and the release of a metal spray. For each ejection regime the driving mechanisms is analyzed, aided by a two-temperature model. Cap ejection is driven by relaxation of thermal stresses induced by laser-induced heating, whereas jet- and spray-ejections are vapor-driven (as the metal film is partly vaporized). We introduce energy balances which provide the ejection velocity in qualitative agreement with our velocity measurements. The threshold fluences separating the ejection regimes are determined. In addition, the fluence threshold below which no ejection is observed is quantitatively described using a balance between the surface energy of the (locally melted) film and its inertia.

\footnotetext{
*Under review: R. Pohl, C.W. Visser, G.W. Römer, D. Lohse, C. Sun, A.J. Huis in 't Veld. (2014)

${ }^{\dagger}$ Equal contribution of RP and CWV. Experiments, setup, theory (all in part), and first draft by CWV.
} 
In conclusion, the ejection type can now be controlled, which allows for improved deposition of pure metal droplets and sprays.

\subsection{Introduction}

Laser-induced forward transfer (LIFT) is a high-resolution 3D direct-write method that was first demonstrated in 1986 [1]. For the LIFT process, a transparent substrate (carrier) is coated with a thin film (donor) and is placed in close proximity to a second substrate (receiver), see figure 5.1. A pulsed laser beam is focused through the carrier onto the carrier-donor-interface. The incident laser pulse is absorbed within a thin layer of the donor material. At sufficiently high laser fluences, the donor material is ejected and deposited on a receiver substrate.

LIFT has a high potential for printing of various materials (including pure metals [2-7]) which cannot be deposited using conventional methods such as ink-jet printing, while retaining key advantages including high resolution (down to $300 \mathrm{~nm}$ [8]), and mask-less, contact-free deposition at room conditions. In particular, the deposition of pure-metal droplets in the liquid phase allows for deposition of conductive patterns [9, 10], from which the semiconductor industry could strongly benefit [11]. However, despite process improvements in various ways [12-16], the high potential of LIFT for liquid-metal deposition has not been met as the deposited features are poorly controlled. This for example results in deposition of one main droplet surrounded by smaller satellite droplets, the deposition of many particles [8], or a significant uncertainty in the deposition location due to a limited control of the ejection angle [17].

Improving LIFT is far from straightforward, as the ejection process has hardly been visualized due to the extremely short time of the process and, consequently, is poorly understood. The ejection time scale is estimated to be only $\tau \sim V / L \sim 100 \mathrm{~ns}$, assuming a velocity $V \approx 100 \mathrm{~m} / \mathrm{s}$ and a length scale $L \approx 10 \mu \mathrm{m}$ [18], resulting in challenging visualization conditions. So far, time-resolved visualization has been achieved for relatively thick liquid-film [19-23] and solid-phase [24-26] or pastetransfer [27, 28] processes. Observations of LIFT-processing of $\mathrm{Au}$ [29], $\mathrm{Ni}$ [30], $\mathrm{Al}$ [31], and $\mathrm{Cr}$ [32] do not provide sufficient spatial resolutions to track the process in detail. Therefore, theories describing the ejection mechanism have been proposed based on the craters left in the donor layer or deposited features on the receiver substrate [33-35]. In addition, numerical simulations have been performed [36-38]. Two driving mechanisms of the ejection process are commonly proposed (for these and more theories see [33, 34, 39]). First, relaxation of thermally induced stresses [40] could drive the ejection. Second, partial evaporation [39] of the donor layer, 


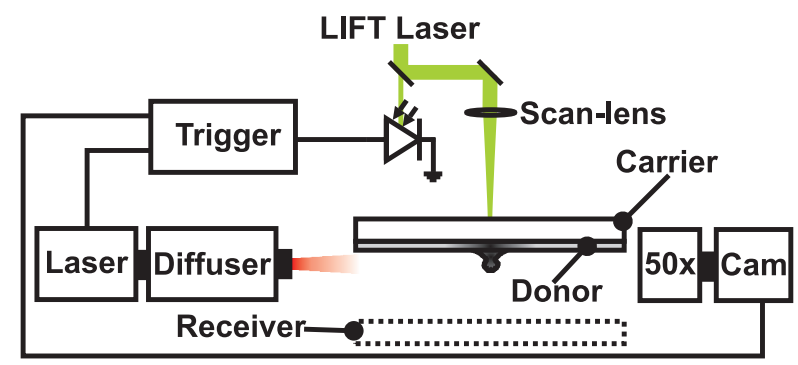

Figure 5.1: Sketch of the experimental setup. The experiments were conducted without a receiving substrate.

resulting in the formation of an expanding vapor bubble, may accelerate and eject the donor material. However, as yet, it is unknown under which conditions these ejection mechanisms occur.

Here we visualize and describe different types of ejections occurring in LIFT of copper and gold films. This allows to optimize the process parameters for specific applications, such as metal micro-manufacturing. To this aim, high-speed, highresolution visualization of ejection events in picosecond LIFT is pursued, revealing three ejection regimes and corresponding ejection velocities. We interpret the experimental results using a two-temperature model, providing key evidence for the underlying ejection mechanism. Based on this evidence, the energy balances required to model the ejection regime and ejection velocity are proposed. This approach provides the key parameter settings for each regime, and provides a simple estimate of the ejection fluence threshold.

The experimental and numerical methods are discussed in section 5.2. Highresolution images of the ejection dynamics and two-temperature model calculations are presented in section 5.3, as well as the physical interpretation of these results. The implications and limitations of these results are discussed in section 5.4, followed by the conclusions in section 5.5.

\subsection{Methods}

\subsubsection{Experimental Setup}

The experiments are performed using the setup schematically shown in figure 5.1. For LIFT, a Yb:YAG laser source with a fixed pulse duration of $6.7 \mathrm{ps}$, a wavelength of $515 \mathrm{~nm}$ (SHG) and a Gaussian beam profile with a beam quality factor of $M^{2} \leq 1.3$ was used. The beam was focused onto the carrier-donor interface using a f-theta- 
ronar scan-lens with a focal length of $100 \mathrm{~mm}$. The beam waist $\left(1 / \mathrm{e}^{2}\right)$ was measured to be $8.3 \pm 0.6 \mu \mathrm{m}$. The fluence values in this article represent peak fluences. The maximum error in the fluence is $20 \mathrm{~mJ} / \mathrm{cm}^{2}$, as determined using the $\mathrm{D}^{2}$-Method [41]. Copper and gold films with thicknesses $50 \mathrm{~nm}<d<400 \mathrm{~nm}$ (magnetron sputtered at $23 \mathrm{~nm} / \mathrm{m}$ onto a $1 \mathrm{~mm}$ thick glass carrier) are used as donor layer. To optimize the experimental imaging conditions, the receiving substrate was omitted.

Images were recorded using a dual-shot CCD camera (PCO Sensicam), mounted to a microscope with a $50 \times$ long-distance objective. Brightfield flash illumination was provided by a dual-cavity Nd:YAG laser with a pulse duration of $6 \mathrm{~ns}$. A highefficiency diffuser was used to diffuse this laser pulse and thereby prevent fringes. To determine the ejection velocity, the distance between the ejection crater in the donor layer and the tip of the ejection was measured and divided over the time between the ejection and the frame illumination. For triggering, a pulse delay-generator was used (Berkeley Nucleonics BNC 575). The trigger sequence was started by the output of a photo diode exposed to the LIFT laser beam path. All components were selected to achieve a high temporal resolution, resulting in a temporal measurement error of $10 \mathrm{~ns}$. The spatial resolution of the imaging system is limited by the diffraction limit or motion blur (for ejection velocities exceeding $100 \mathrm{~m} / \mathrm{s}$ ).

\subsubsection{Two-Temperature Model}

As the laser pulse duration is comparable to the time scale of the electron-phonon relaxation [42], a two-temperature model (TTM) is used to describe the lattice temperature $T_{l}$ and the electron temperature $T_{e}$ of the donor layer. Hence, the temperature evolution of the electron- and phonon-subsystems are modeled by the following set of differential equations [43]:

$$
C_{e} \frac{\delta T_{e}}{\delta t}=\frac{\delta}{\delta z} K_{e} \frac{\delta T_{e}}{\delta z}-g\left(T_{e}-T_{l}\right)+S,
$$

and

$$
C_{l}\left(T_{l}\right) \frac{\delta T_{l}}{\delta t}=g\left(T_{e}-T_{l}\right),
$$

where $C_{e}, C_{l}\left(T_{l}\right)$ and $g$ represent the electron heat capacity, the phonon heat capacity, and the electron-phonon coupling factor, respectively. The electron heat capacity is modeled as $C_{e}=A_{e} T_{e}$, where $A_{e}$ is the electron specific heat constant, and the electron thermal conductivity as $K_{e}=K_{e 0} T_{e} / T_{l}$. The enthalpy of melting and vaporization are incorporated by adding a Gaussian function to the heat capacity, centered at the equilibrium phase-change temperatures, with a standard deviation of $20 \mathrm{~K}$. The 
integration of those functions yields the phase-change enthalpies. The source term $S$ describes the electron heating by Lambert-Beer absorption for thin films:

$$
S=\alpha(1-R) I_{0}(t) \frac{\exp (-\alpha z)}{1-\exp (-\alpha d)}
$$

where $R$ denotes the reflection coefficient and $\alpha$ the linear absorption coefficient. Transmitted light is excluded by the denomiator in equation (5.3) $(1-\exp (-\alpha d))$, which is relevant here since thin films are used [43]. The temporal evolution of the laser pulse intensity $I_{0}$ is described by:

$$
I_{0}(t)=\frac{2 F \sqrt{\ln 2}}{\sqrt{\pi} \tau_{p}} \exp \left(-4 \ln 2\left(t / \tau_{p}\right)^{2}\right)
$$

Here, $F$ and $\tau_{p}$ are the laser fluence and pulse duration, respectively. The system is numerically solved using the following assumptions and boundary conditions. Superheating might occur, resulting in melting time scales of up to $100 \mathrm{ps}$ [44]. Since these time scales are still significantly shorter than the ejection time scale of our LIFT experiments, temperature homogenization is expected to occur prior to ejection. Therefore, superheating is ignored in the temperature model. Heat conduction into the carrier and the air are ignored (i.e. at $z=0$ and $z=d$ we use $C_{e} \frac{\delta T_{e}}{\delta t}=0$ ). A one-dimensional model is used, as (1) the laser spot size exceeds the film thickness by two orders of magnitude and (2) the thermal penetration length in the lateral dimension of the film remains much smaller than the spot size until $\sim 10 \mathrm{~ns}$ after the laser pulse, during which the material is ejected. The ballistic motion of electrons is ignored, as $\tau_{p}>1 \mathrm{ps}$ [45]. The equations are solved numerically for a time period of 100 ps. The material properties used are summarized in table 5.1.

\subsection{Results}

\subsubsection{Ejection Regime Classification}

Figure 5.2 shows the fluence-resolved ejection dynamics for a $200 \mathrm{~nm}$ copper film. Figures 5.2 (a) - (c) show typical ejections in the low-fluence regime $(F \leq 600$ $\mathrm{mJ} / \mathrm{cm}^{2}$ ), where the ejection of a cap is observed (cap ejection is a novel regime, as discussed in section 5.4). For intermediate fluences $\left(600 \mathrm{~mJ} / \mathrm{cm}^{2} \leq F \leq 740\right.$ $\mathrm{mJ} / \mathrm{cm}^{2}$ ), a jet-like feature is formed on the apex of the ejected cap, see figure 5.2 (d) and (e). At even higher fluence levels $\left(F \geq 740 \mathrm{~mJ} / \mathrm{cm}^{2}\right)$, ejection of a spray is observed, see Figure 5.2 (f) and (g). These sprays are characterized by a cloud of particles or droplets, instead of the more coherent features observed in the cap ejection regime. 

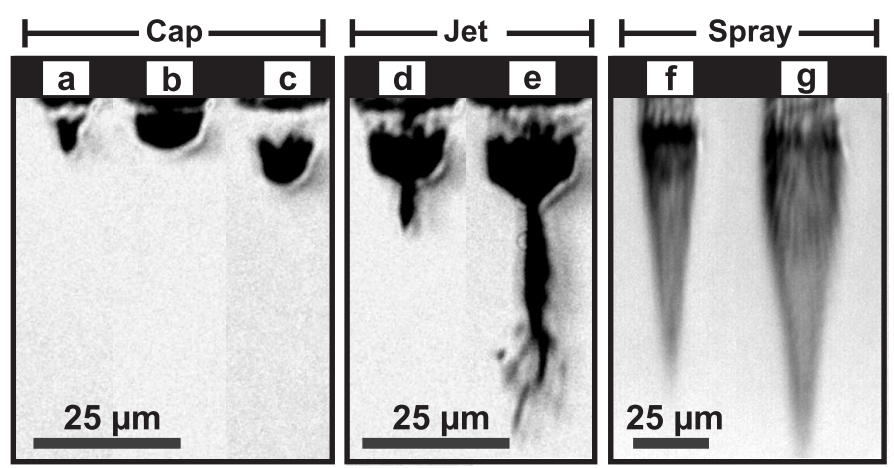

Figure 5.2: Ejection of a $200 \mathrm{~nm}$ copper film, visualized $125 \mathrm{~ns}$ after the incident laser pulse. Figure (a) to (g) correspond to increasing fluence values of 314, 392, 480, 660, 864, 1060, and $1576 \mathrm{~mJ} / \mathrm{cm}^{2}$, respectively. The black bar at the top of the image schematically shows the location of the donor layer. Images (a) to (c) illustrate the cap ejection regime observed for low fluences; (d) and (e) show the formation of a jet from the apex of the cap for intermediate fluences, and (f) and (g) show the ejection of a fast copper spray. In image (g), some droplets are visible as lines, as their high velocity results in strong motion blur (the tip speed is 700 $\mathrm{m} / \mathrm{s})$.

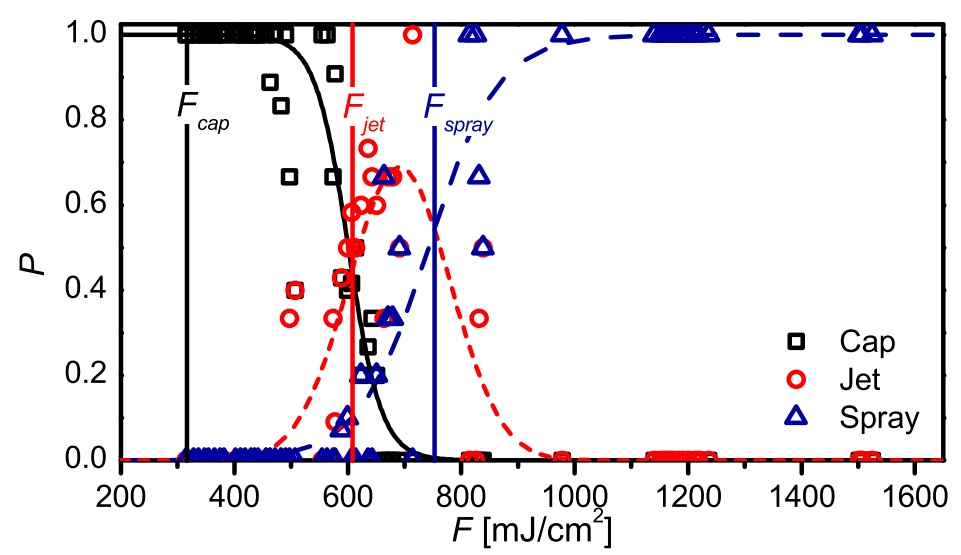

Figure 5.3: Probability $P$ of the type of ejections observed (cap, jet or spray) versus the incident laser fluence. 


\begin{tabular}{|c|l|c|c|c|}
\hline Sym & Property & Copper & Gold & Unit \\
\hline$A_{e}$ & Electron specific heat constant & 98 & 71 & $\mathrm{~J} \mathrm{~m}^{-3} \mathrm{~K}^{-2}$ \\
\hline$C_{l}$ & Lattice heat capacity & $3.26 \cdot 10^{6}$ & $2.19 \cdot 10^{6}$ & $\mathrm{~J} \mathrm{~m}^{-3} \mathrm{~K}^{-1}$ \\
\hline$d$ & Donor layer thickness & 50 to 200 & 100 to 400 & $\mathrm{~nm}$ \\
\hline$E$ & Young's modulus & 110 & 74 & $\mathrm{GPa}$ \\
\hline$g$ & Electron-phonon coupling factor & $10 \cdot 10^{16}$ & $2.1 \cdot 10^{16}$ & $\mathrm{~W} \mathrm{~m}{ }^{-3} \mathrm{~K}^{-1}$ \\
\hline$H_{m}$ & Melting enthalpy & $1.9 \cdot 10^{9}$ & $1.14 \cdot 10^{9}$ & $\mathrm{~kJ} / \mathrm{kg}^{-1}$ \\
\hline$K_{e 0}$ & Electron heat conductivity & 401 & 318 & $\mathrm{~W} \mathrm{~m}{ }^{-1} \mathrm{~K}^{-1}$ \\
\hline$R$ & Reflection coefficient & 0.6 & 0.64 & - \\
\hline$T_{m}$ & Melting temperature & 1358 & 1337 & $\mathrm{~K}$ \\
\hline$T_{v}$ & Vaporization temperature & 2835 & 2800 & $\mathrm{~K}$ \\
\hline$\alpha$ & Absorption coefficient & $6.33 \cdot 10^{5}$ & $5.2 \cdot 10^{5}$ & $\mathrm{~cm}^{-1}$ \\
\hline$\alpha_{t h}$ & Coefficient of thermal expansion & $16.5 \cdot 10^{-6}$ & $14.2 \cdot 10^{-6}$ & $\mathrm{~K}^{-1}$ \\
\hline$v$ & Poisson ratio & 0.35 & 0.43 & - \\
\hline$\rho$ & Density & 8960 & $17 \cdot 10^{3}$ & $\mathrm{~kg} \mathrm{~m}^{-3}$ \\
\hline$\sigma$ & Surface tension & 1.2 & 1 & $\mathrm{~N} \mathrm{~m}^{-1}$ \\
\hline$\tau_{p}$ & Laser pulse duration & 6.7 & 6.7 & $\mathrm{ps}^{-1}$ \\
\hline
\end{tabular}

Table 5.1: Material properties used [42, 45].

Establishing the threshold fluences between these regimes proved challenging, as experiments with the same input parameter settings sometimes resulted in different regimes (in particular close to the transition fluences). Therefore, the incidence rate of each regime was binned as a function of fluence. For the $200 \mathrm{~nm}$ copper film, 388 ejection events were categorized and binned (using a bin width of $10 \mathrm{~mJ} / \mathrm{cm}^{2}$ ). Figure 5.3 shows the probability of each ejection regime for each bin. The lower fluence thresholds for the cap-, jet- and spray-regimes were based on a regime incidence of $50 \%$, yielding values of 320,600 , and $740 \mathrm{~mJ} / \mathrm{cm}^{2}$, respectively. These thresholds are connected to the film temperature (discussed next), providing evidence for the ejection driving mechanism.

\subsubsection{Temperature Analysis}

The temporal evolution and spatial distribution of the electron- and lattice temperatures are computed with the model described in section 5.2. Example results are plotted in figure 5.4, showing the interface lattice temperatures and the electron temperature as a function of time. First, the laser pulse is absorbed by the electron subsystem in the optical absorption depth, which has a thickness of $1 / \alpha \approx 15 \mathrm{~nm} \ll d$, 


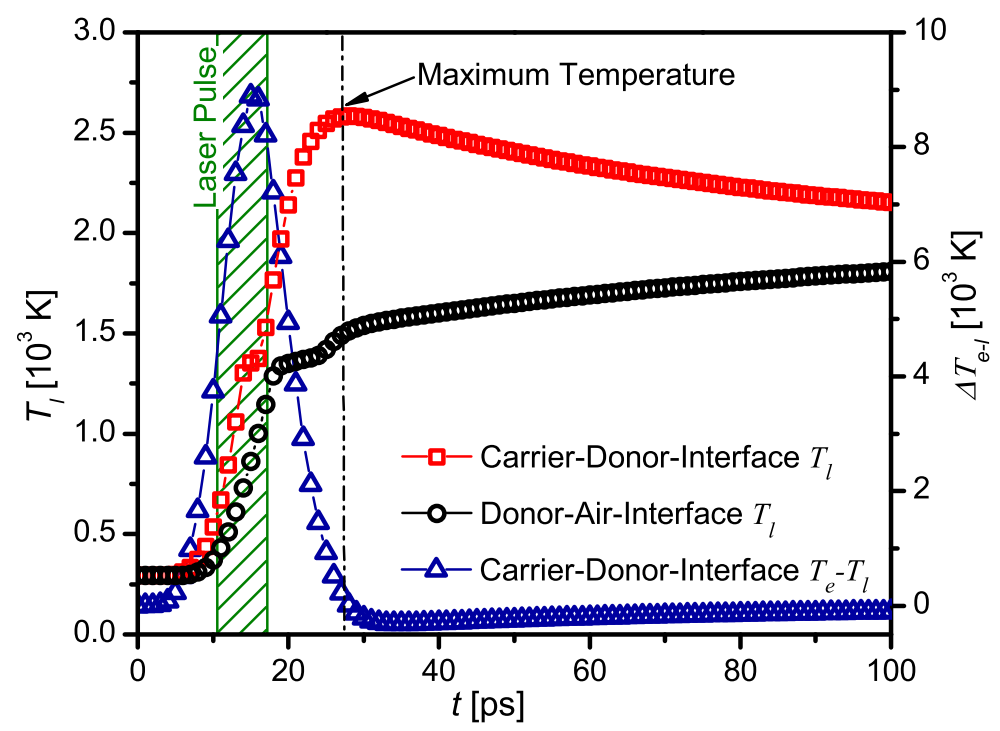

Figure 5.4: Calculated lattice temperature at the carrier-donor interface (squares, left axis) and the donor-air interface (circles, left axis) as a function of time (at $t=0$ the simulation starts; the peak pulse energy is reached at $t=2 \tau_{p}=13 \mathrm{ps}$ ), for $F=376 \mathrm{~mJ} / \mathrm{cm}^{2}$. The hashed area indicates the full width half max of the laser pulse duration. The electron-phonon temperature difference at the carrier-donor-interface (triangles, right axis) illustrates the high electron temperatures reached and the electron cooling on the electron-phonon temperature relaxation time scale $(\sim 20 \mathrm{ps})$.

resulting in electron temperatures up to $\sim 10^{4} \mathrm{~K}$ at the carrier-donor interface (red squares in figure 5.4). Subsequently, the lattice is heated by the electrons and reaches a peak temperature of $\sim 2600 \mathrm{~K}$ on the relaxation time of the electron-phonon system $\left(\tau_{e p} \sim 20 \mathrm{ps}\right)$. An almost homogeneous temperature is reached after $t \approx 100$ ps, corresponding to the thermal diffusion time scale $\tau_{t h}=d^{2} /\left(2 \alpha_{D}\right) \approx 180 \mathrm{ps}$, with $\alpha_{D}=1.1 \cdot 10^{-4} \mathrm{~m}^{2} / \mathrm{s}$ the thermal diffusivity.

Figure 5.5 shows the calculated maximum interface temperatures of the metal film as a function of the laser fluence. The temperature plateaus at $T \approx 1400 \mathrm{~K}$ and $T \approx 2800 \mathrm{~K}$ indicate the melting- and evaporation phase changes, respectively. Here, increasing the fluence only results in the phase change of a larger material fraction. The onset of melting (i.e. partial melting of metal close to the carrier-metal interface) is predicted to occur at $F \approx 120 \mathrm{~mJ} / \mathrm{cm}^{2}$, until at a fluence of $F \approx 350 \mathrm{~mJ} / \mathrm{cm}^{2}$ the film is completely melted. The onset of evaporation (i.e. partial evaporation of metal close to the carrier-metal interface) occurs at $F \approx 400 \mathrm{~mJ} / \mathrm{cm}^{2}$. For fluences $\geq 610 \mathrm{~mJ} / \mathrm{cm}^{2}$, the full layer has reached the steady-state vaporization temperature and increasing the 


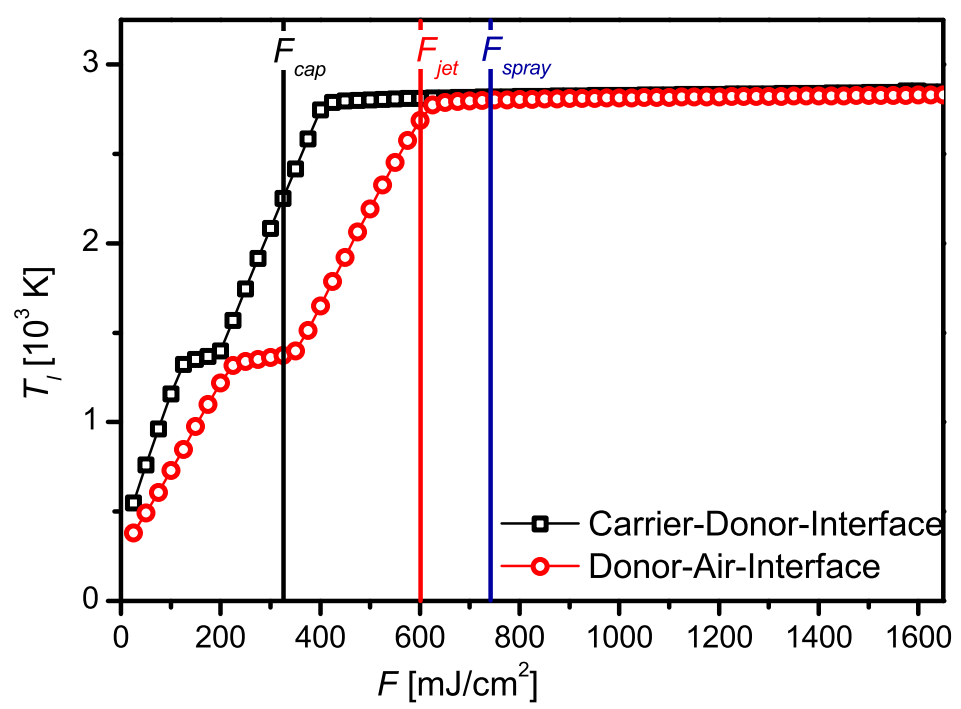

Figure 5.5: Computed maximum temperature as function of laser fluence, at the interfaces of a $200 \mathrm{~nm}$ copper film.

fluence only results in a larger vaporized fraction of the film. A further temperature increase is expected only for fluences resulting in complete film vaporization, which are beyond the current parameter space.

The transition fluences, separating the ejection regimes, are re-plotted in figure 5.5. The ejection threshold for cap ejection $F_{c a p}=320 \mathrm{~mJ} / \mathrm{cm}^{2}$ coincides with the full melting of the donor film. The transition to the jet ejection regime occurs when the full layer reaches the evaporation temperature (in subsection 5.3.3 we will argue that the driving mechanism of the jet and the spray are equal).

This quantitative correspondence between the phase changes and the threshold fluences for cap and jet ejection suggests two different ejection mechanisms. This will be discussed in the next section as follows:

- Cap ejection by thermally-induced stress relaxation (subsection 5.3.3 (a)).

- No ejection for sub-threshold fluences, due to surface tension retracting the cap (subsection 5.3.3 (b)).

- Jet-and spray ejection by partial film vaporization (subsection 5.3.3 (c)). 

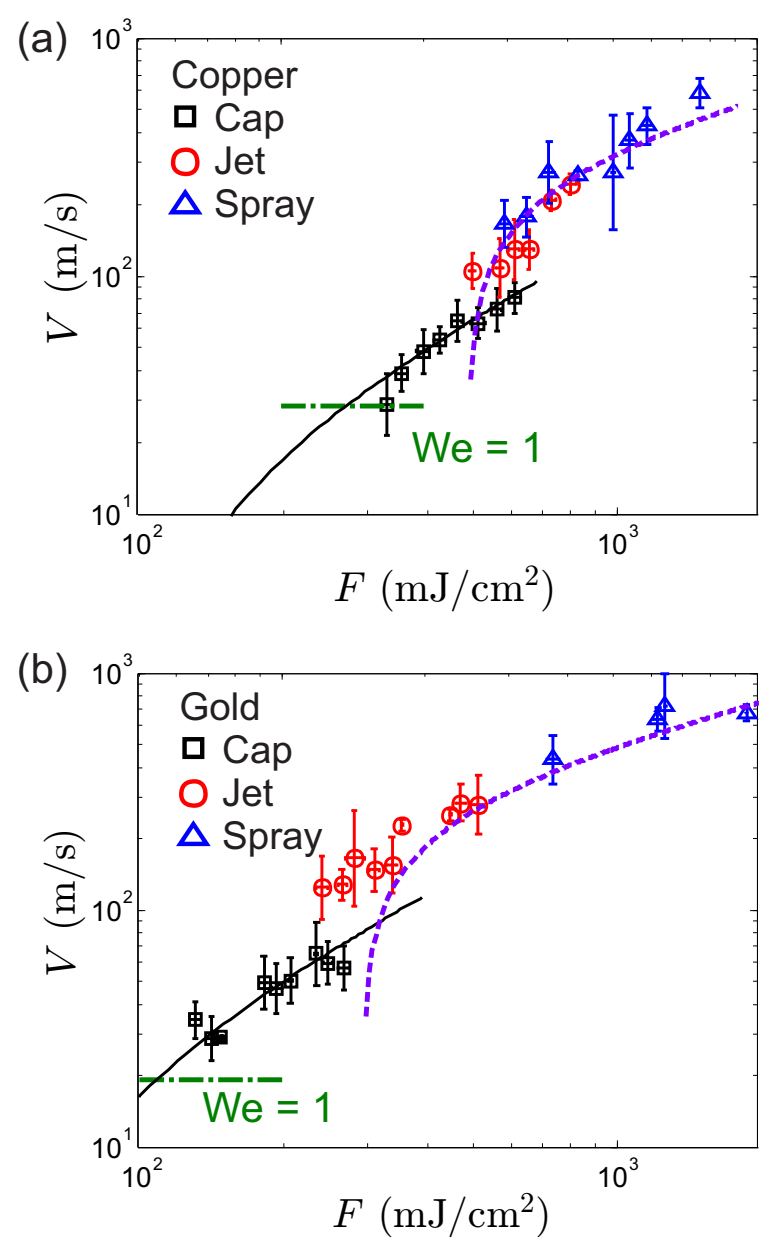

Figure 5.6: Ejection velocity as a function of the laser fluence, for a $200 \mathrm{~nm}$ copper film (a) and a $160 \mathrm{~nm}$ gold film (b). The different markers indicate the ejection regimes analogous to figure 5.2. The green dash-dotted line indicates the predicted minimum velocity; the black solid lines show the modeled cap ejection velocity (equation (5.9)), and the dashed purple line indicates the spray- and jet ejection velocity model (equation (5.15)). The displayed data points represent grouped measurements with error bars indicating the standard deviation (each data point consists of at least 10 individual measurements). 


\subsubsection{Ejection Mechanisms}

Cap Ejection by thermally-induced stress relaxation As shown in figure 5.5, vaporization is not predicted by our temperature model just above the cap ejection fluence threshold. Therefore, thermal compression of the metal film and subsequent release of elastic energy is proposed as the driving mechanism analogous to Refs. $[34,38]$. The elastic energy is modeled as

$$
E_{E}=\int_{0}^{d} A K u^{2} d z \approx \frac{1}{2} d A K \alpha_{t h}^{2} \Delta T^{2},
$$

using thermal expansion $u=\alpha_{t h} \Delta T$ with $\alpha_{t h}$ the thermal expansion coefficient and $\Delta T$ the lattice temperature increase by the laser pulse, a bulk modulus $K=\frac{E}{3(1-2 v)}$ with Young's modulus $E$ and the Poisson ratio $v$, and a surface area $A$. For a laser pulse resulting in homogeneous heating over the film thickness, this yields

$$
E_{E}=\frac{1}{2} d A K \alpha_{t h}^{2} \Delta T^{2}
$$

with the temperature increase modeled as

$$
\Delta T=\frac{(1-R) F-d H_{m}}{d C_{l}},
$$

with $H_{m}$ the melting enthalpy, $R$ the reflection coefficient, and $C_{l}$ the lattice heat capacity. By equating this elastic energy to the kinetic energy

$$
E_{\text {kin }}=\frac{1}{2} \rho d A v^{2}
$$

where $\rho$ is the density, the velocity in the elastic regime is derived as:

$$
V=\alpha_{t h} C_{0} \Delta T \sqrt{\frac{K}{\rho}}
$$

with $C_{0}$ a fitting prefactor. The resulting velocities are shown by the solid lines in figures 5.6 (a) for copper (with $C_{0}=0.35$ ) and 5.6 (b) for gold (with $C_{0}=0.7$ ). Using prefactors of order one, good agreement between the model and the measurements is obtained. 
Threshold ejection fluence governed by the capillary-inertial energy balance Surprisingly, only velocities exceeding $\sim 20 \mathrm{~m} / \mathrm{s}$ are observed even just above the ejection threshold, as shown in figure 5.6. Previous reports have shown that for subejection threshold fluences the film is accelerated, but retracted by surface tension before it can escape the (liquid) donor layer [22]. This suggests that ejection only takes place if the kinetic energy of the ejected material exceeds its surface energy. Dividing the kinetic energy $E_{k i n}$ by the surface energy $E_{s}$ provides

$$
\frac{E_{k i n}}{E_{s}} \sim \frac{\rho d v^{2}}{\sigma}=\mathrm{We},
$$

with $v$ the maximum tip velocity, $\sigma$ the surface tension of molten copper, and We the Weber number, i.e. the ratio of inertial energy and surface energy. Using a threshold Weber number $\mathrm{We}=1$, the minimal ejection velocity is readily determined from equation (5.10). As shown in figure 5.6, this velocity reasonably matches our measured minimum velocities.

Using the condition $\mathrm{We}=1$, the minimum cap ejection fluence is readily determined. Combining equations (5.7), (5.9), (5.10), and solving for the fluence provides:

$$
F_{\text {cap }}=\frac{1}{1-R}\left[\frac{\rho d C_{l}}{\alpha_{t h} C_{0}} \sqrt{\frac{\sigma}{K d}}+d H_{m}\right],
$$

where again for gold $C_{0}=0.7$ and for copper $C_{0}=0.35$ are used. The threshold fluence is plotted as a function of the film thickness in figure 5.7, showing good agreement to the measured data for copper and gold films of various thicknesses. This suggests that the condition $\mathrm{We}=1$ provides a simple and robust criterion for determination of the threshold fluence for LIFT of (locally) liquid metal films.

Jet- and Spray Ejection by partial film vaporization For both jet- and spray ejection, vaporization is predicted within the whole film (see figure 5.5). Therefore, vapor-driven ejection is assumed for these regimes ${ }^{\ddagger}$. The laser energy heating the vapor is estimated as the initial energy minus the energy required for melting of the film and heating it to the boiling temperature. This provides the energy contained by the vapor as:

$$
E_{\text {vap }}=C_{1} A\left((1-R) F-d \cdot H_{m}-d \cdot C_{l}\left(T_{v}-T_{0}\right)\right),
$$

\footnotetext{
$¥$ The different footprints of these regimes (observed from figure 5.2) are likely due to the Gaussian spatial distribution of the laser pulse: in the jetting regime vaporization only occurs in a small spot in the center of the melt area, whereas in the spray ejection regime this occurs in almost the full melt area. The similarity of the jet- and spray regimes is confirmed by the partial overlap of the jet- and spray-ejection velocities (figure 5.6), and therefore no distinction is made regarding the driving mechanism.
} 


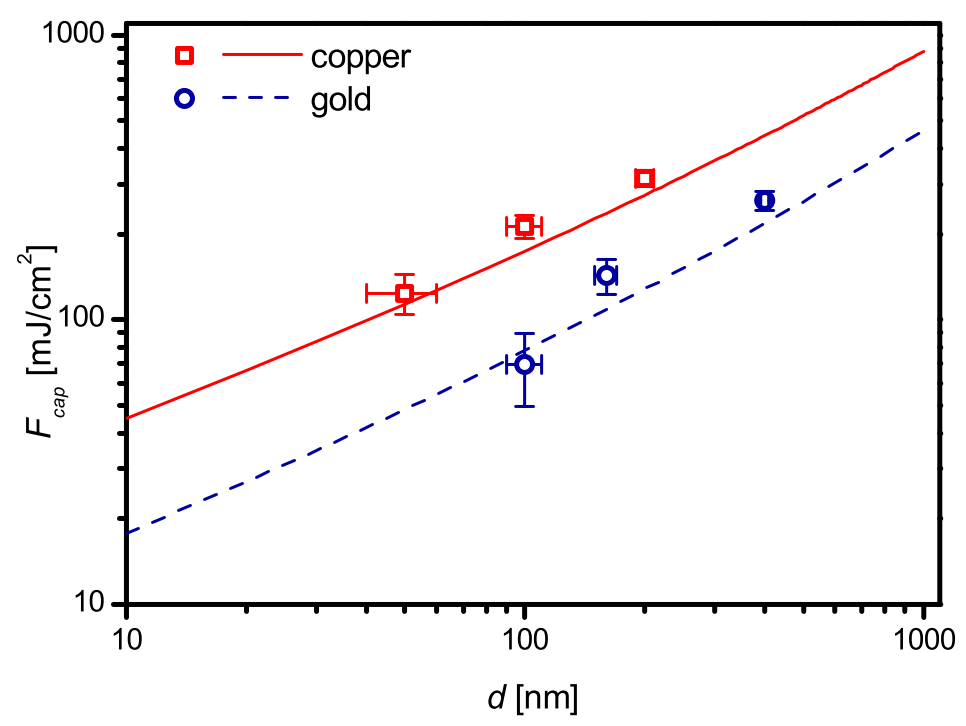

Figure 5.7: Threshold ejection fluence $F_{\text {cap }}$ as a function of the film thickness, for copper and gold films. The markers indicate measured values, the lines indicate the model prediction (equation (5.11)).

where $T_{0}$ is the initial (room) temperature, $T_{v}$ is the boiling temperature and $C_{1}$ is a prefactor. Equating this to the kinetic energy (equation (5.8)), the ejection velocity is obtained:

$$
V=\sqrt{\frac{2 C_{1}\left((1-R) F-d \cdot H_{m}-d \cdot C_{l}\left(T_{v}-T_{0}\right)\right)}{\rho d}} .
$$

Figure 5.6 shows the predicted velocities (purple dashed lines) for copper (figure (a), using $C_{1}=0.05$ ) and gold (figure (b), using $C_{1}=0.12$ ). Although prefactors are required for quantitative agreement, the transition from the cap- to the jet-ejection regime (which is hardly influenced by the prefactor) is captured. The non-unity value of $C_{1}$ may be due to a subtlety of the vaporization enthalpy, which partly consists of the energy required for the atom-by-atom escape through the liquid surface, and partly of the work done by the expanding vapor [46]. At room conditions, the work done by the expanding vapor (which we consider as the driving mechanism) is only $8 \%$ of the total vaporization enthalpy for both copper and gold, i.e. of similar order as the prefactors used. Therefore, using only the work done by the expanding vapor (as for example provided in Ref. [46]) may allow for a reasonable velocity estimate. where again for gold $C_{0}=0.7$ and for copper $C_{0}=0.35$ are used. The threshold fluence is plotted as a function of the film thickness in figure 5.7, showing good 
agreement to the measured data for copper and gold films of various thicknesses. This suggests that the condition $\mathrm{We}=1$ provides a simple and robust criterion for determination of the threshold fluence for LIFT of (locally) liquid metal films.

Jet- and Spray Ejection by partial film vaporization For both jet- and spray ejection, vaporization is predicted within the whole film (see figure 5.5). Therefore, vapor-driven ejection is assumed for these regimes ${ }^{\S}$. The laser energy heating the vapor is estimated as the initial energy minus the energy required for melting of the film and heating it to the boiling temperature. This provides the energy contained by the vapor as:

$$
E_{\text {vap }}=C_{1} A\left((1-R) F-d \cdot H_{m}-d \cdot C_{l}\left(T_{v}-T_{0}\right)\right),
$$

where $T_{0}$ is the initial (room) temperature, $T_{v}$ is the boiling temperature and $C_{1}$ is a prefactor. Equating this to the kinetic energy (equation (5.8)), the ejection velocity is obtained:

$$
V=\sqrt{\frac{2 C_{1}\left((1-R) F-d \cdot H_{m}-d \cdot C_{l}\left(T_{v}-T_{0}\right)\right)}{\rho d}} .
$$

Figure 5.6 shows the predicted velocities (purple dashed lines) for copper (figure (a), using $C_{1}=0.05$ ) and gold (figure (b), using $C_{1}=0.12$ ). Although prefactors are required for quantitative agreement, the transition from the cap- to the jet-ejection regime (which is hardly influenced by the prefactor) is captured. The non-unity value of $C_{1}$ may be due to a subtlety of the vaporization enthalpy, which partly consists of the energy required for the atom-by-atom escape through the liquid surface, and partly of the work done by the expanding vapor [46]. At room conditions, the work done by the expanding vapor (which we consider as the driving mechanism) is only $8 \%$ of the total vaporization enthalpy for both copper and gold, i.e. of similar order as the prefactors used. Therefore, using only the work done by the expanding vapor (as for example provided in Ref. [46]) may allow for a reasonable velocity estimate.

In the spray ejection regime, the speed of sound in air is generally exceeded by the ejected material, and ejection-induced shock waves must be present. These shock waves were observed only occasionally and were faint (not shown here). Since the shock waves do not seem to influence the ejection regime or velocity, and detailed

\footnotetext{
$\S$ The different footprints of these regimes (observed from figure 5.2) are likely due to the Gaussian spatial distribution of the laser pulse: in the jetting regime vaporization only occurs in a small spot in the center of the melt area, whereas in the spray ejection regime this occurs in almost the full melt area. The similarity of the jet- and spray regimes is confirmed by the partial overlap of the jet- and spray-ejection velocities (figure 5.6), and therefore no distinction is made regarding the driving mechanism.
} 
visualization requires a different experimental setup, we refer to refs. [47, 48] for a for a detailed discussion of shock waves.

\subsection{Discussion}

Liquid-phase cap ejection is scarcely described ejection regime in LIFT [49], which we therefore concisely discuss. Cap ejection strongly resembles a nanobump torn off around its base [50-52] (a nanobump is a smooth bump in the metal film, which is observed below the ejection threshold fluence), which is distinctly different from the commonly reported LIFT ejection by formation and break-up of a liquid filament $[17,40]$. The dynamics of liquid-phase nanobump formation were recently investigated using molecular dynamics simulations, revealing extreme thinning of the donor film at the base of the bump [38]. Cap ejection was not reported there, since the simulations were limited to short time scales or sub-ejection fluences. However, rupture of this thin part of the film is easily conceivable and would directly correspond to our cap ejections. Since liquid film rupture is likely to result in the formation of multiple droplets, this mechanism may also explain the formation of satellite droplets generally observed [49]. Despite the multi-droplet formation, the cap ejection regime has a high potential for $3 \mathrm{D}$ additive manufacturing, since the ejected cap contracts into a main droplet with a well-defined size and speed.

The cap ejection threshold fluence is accurately captured by the capillary-inertial energy balance resulting in equation (5.5). The generality of this energy balance is now assessed using the (rare) literature data on the liquid-film LIFT ejection. These references include velocity data below and above the ejection threshold. First, for numerical work on LIFT of Newtonian liquid films deposited on a dynamic release layer [22], a threshold ejection Weber number $\mathrm{We}_{t h}=1.1$ is obtained in agreement with the $\mathrm{We}=1$ criterion proposed here. In a second case [19], no detachment was observed even for experiments performed at We $\approx 80$. However, in that case, rheological modifiers were added to the viscous film, potentially delaying film break-up as compared to Newtonian fluids. This suggest that $\mathrm{We}=1$ sets the lower bound of the minimum ejection velocity: for We $<1$ retraction is expected, whereas for We $>1$ ejection occurs only if the film breaks up on a sufficiently short time scale.

Elastic stress release and partial vaporization of the film are well-established driving mechanisms for metal LIFT [33, 34, 36-38, 43]. However, the actual driving mechanisms are more complex. For example, numerical simulations revealed that cap-ejection regime is the result of a heat-induced (but still elastic) pressure wave traveling perpendicular to the film [36-38, 43]. This wave is reflected at the donor- 
air surface and induces pull-off of the donor from the carrier "I . Also superheating and vapor formation, expansion, and condensation could occur [54]. In this view, it is remarkable that our simple energy balances seems to capture these phenomena, although a prefactor is required for quantitative agreement to the measurements.

Still, even for a single driving mechanism, actual observations may depend on the pulse duration and the film thickness. For example, for nanosecond pulse durations, film deformation and thermal diffusion are significant already during the pulse. Therefore, the energy deposition will be less confined and shock waves within the film could be diminished, resulting in lower ejection velocities [17]. For femtosecond pulses, the energy absorption by the electrons is much faster than for our picosecond pulses. However, in both cases, the energy transfer into the lattice is limited by the time scale at which the hot electrons heat the lattice (i.e. the electron-phonon coupling time scale), which usually is in the order of $\sim 10$ picoseconds. Therefore, in this short-pulse regime, lattice heating is hardly affected [55] and our models are expected to be valid. Similarly, the thickness of the film may strongly affect the observed ejection regime. In particular, if the optical penetration length is smaller than the film thickness and ejection occurs prior to thermal diffusion over the film thickness, solid-state ejection is observed [25]. In these cases the ejection threshold depends on the yield strength of the film or its adhesion to the carrier substrate $[33,34]$, and equation (5.5) no longer applies.

\subsection{Conclusions}

High-resolution images of donor ejection during picosecond LIFT are presented. Varying the fluence $F$ reveals different ejection regimes, which are illustrated for a $200 \mathrm{~nm}$ copper film. For $160 \mathrm{~mJ} / \mathrm{cm}^{2}<F<300 \mathrm{~mJ} / \mathrm{cm}^{2}$, ejection of a hemispherical piece of the film is observed. This is a scarcely addressed regime that we call "cap"ejection. For $300 \mathrm{~mJ} / \mathrm{cm}^{2}<F<370 \mathrm{~mJ} / \mathrm{cm}^{2}$ "jet-ejection" occurs, since here a narrow jet leading the cap apex is observed. For $F>370 \mathrm{~mJ} / \mathrm{cm}^{2}$ a cloud of particles is observed, called "spray ejection". Using the two-temperature model, these regimes are connected to phase changes within the donor layer. In the cap ejection regime, the relaxation of elastic stresses within the (melted) donor film is proposed as the driving mechanism. In the jet- and spray-ejection regimes, for which the driving mechanism

\footnotetext{
IINote that stress release towards the non-heated region of the film is limited by the speed of sound: during the laser pulse duration, the radius of the heated area $(10 \mu \mathrm{m})$ significantly exceeds the distance the compression wave travels through the film $(<1 \mu \mathrm{m})$. Therefore, film buckling is not due to lateral compression, but due to (still elastic [36]) pressure wave reflection. These reflections have been incorporated in more comprehensive semi-analytic models, see for example Ref. [53].
} 
is similar, the expansion of a vapor bubble drives the ejection. These mechanisms are captured by energy balances which provide velocity predictions. Good agreement with velocity measurements for copper and gold films is obtained, using a materialdependent fitting constant. A minimal ejection velocity of $V \approx 20 \mathrm{~m} / \mathrm{s}$ is found to correspond to a Weber number We $\approx 1$. This suggests that for lower ejection velocities (for which $\mathrm{We}<1$ ), surface tension retracts the liquid film. If the velocity prefactor (which is independent of the film thickness) is known, We $=1$ allows to quantitatively determine the ejection threshold fluence. Vice versa, if the ejection threshold fluence is known, the velocity can be quantitatively determined.

The current characterization of different LIFT ejection regimes may allow for more controlled deposition of micron-sized pure metal droplets. In particular, the novel cap ejection regime may extend the range of achievable droplet sizes- and velocities, which we expect to explore in future work.

\section{References}

[1] J. Bohandy, B. F. Kim, and F. J. Adrian, Metal deposition from a supported metal film using an excimer laser, Journal of Applied Physics 60, 1538 (1986).

[2] P. Mogyorosi, T. Szorenyi, K. Bali, Z. Toth, and I. Hevesi, Pulsed laser ablative deposition of thin metal films, Applied Surface Science 36, 157 (1989).

[3] Z. Toth, T. Szorenyi, and A. Toth, Ar + laser-induced forward transfer (LIFT) : a novel method for micrometer-size surface patterning, Applied Surface Science 69, 317 (1993).

[4] V. Schultze and M. Wagner, Blow-off of Aluminum Films, Applied Physics A 53, 241 (1991).

[5] H. Esrom, J.-Y. Zhang, U. Kogelschatz, and A. J. Pedraza, New approach of a laser-induced forward transfer for deposition of patterned thin metal films, Applied Surface Science 86, 202 (1995).

[6] D. a. Willis and V. Grosu, Microdroplet deposition by laser-induced forward transfer, Applied Physics Letters 86, 244103 (2005).

[7] I. Zergioti, S. Mailis, N. Vainos, P. Papakonstantinou, C. Kalpouzos, C. Grigoropoulos, and C. Fotakis, Microdeposition of metal and oxide structures using ultrashort laser pulses, Applied Physics A 66, 579 (1998).

[8] D. P. Banks, C. Grivas, J. D. Mills, R. W. Eason, and I. Zergioti, Nanodroplets deposited in microarrays by femtosecond Ti:sapphire laser-induced forward transfer, Applied Physics Letters 89, 193107 (2006).

[9] C. Germain, L. Charron, L. Lilge, and Y. Y. Tsui, Electrodes for microfluidic devices produced by laser induced forward transfer, Applied Surface Science 253, 8328 (2007).

[10] G. Oosterhuis, A. Prenen, and A. Huis in't Veld, Laser Induced Forward Transfer of Interconnects for $3 D$ Integration, ECS transactions 41, 81 (2012).

[11] F. Roozeboom, M. Smets, B. Kniknie, M. Hoppenbrouwers, G. Dingemans, W. Keuning, W. Kessels, R. Pohl, and A. Huis in 't Veld, 46th IMAPS International Symposium on Microelectronics (IMAPS, Orlando, FL, USA, 2013).

[12] A. Pique, D. Chrisey, R. Auyeung, J. Fitz-Gerald, H.D.Wu, R. McGill, S. Lakeou, P.K.Wu, 
V.Nguyen, and M. Duignan, A novel laser transfer process for direct writing of electronic and sensor materials, Applied Physics A 69, 279 (1999).

[13] B. Hopp, T. Smausz, Z. Antal, N. Kresz, Z. Bor, and D. Chrisey, Absorbing film assisted laser induced forward transfer of fungi (Trichoderma conidia), Applied Physics 96, 3478 (2004).

[14] G. B. Blanchet, Y.-L. Loo, J. A. Rogers, F. Gao, and C. R. Fincher, Large area, high resolution, dry printing of conducting polymers for organic electronics, Applied Physics Letters 82, 463 (2003).

[15] Z. Kantor and T. Szorenyi, Dynamics of long-pulse laser transfer of micrometer-sized metal patterns as followed by time-resolved measurements of reflectivity and transmittance, Applied Physics 78, 2775 (1995).

[16] H. Fukumura, Y. Kohji, K.-I. Nagasawa, and H. Masuhara, Laser Implantation of Pyrene Molecules into Poly(methyl methacrylate) Films, American Chemical Society 116, 10304 (1994).

[17] M. P. Giesbers, M. B. Hoppenbrouwers, E. C. P. Smits, and R. Mandamparambil, in Proceedings of SPIE, , 9135, 91350Z (2014).

[18] M. Versluis, High-speed imaging in fluids, Experiments in Fluids 54, 1458 (2013).

[19] D. Young, R. C. Y. Auyeung, A. Pique, D. B. Chrisey, and D. Dlott, Plume and jetting regimes in a laser based forward transfer process as observed by time-resolved optical microscopy, Applied Surface Science 197-198, 181 (2002).

[20] M. Duocastella, J. Fernandez-Pradas, P. Serra, and J. Morenza, Jet formation in the laser forward transfer of liquids, Applied Physics A 93, 453 (2008).

[21] M. Duocastella, J. M. Fernandez-Pradas, J. L. Morenza, and P. Serra, Time-resolved imaging of the laser forward transfer of liquids, Journal of Applied Physics 106, 084907 (2009).

[22] M. S. Brown, C. F. Brasz, Y. Ventikos, and C. B. Arnold, Impulsively Actuated Jets from Thin Liquid For High-Resolution Printing Applications, Journal of Fluid Mechanics 709, 341 (2012).

[23] C. Boutopoulos, I. Kalpyris, E. Serpetzoglou, and I. Zergioti, Laser-induced forward transfer of silver nanoparticle ink: time-resolved imaging of the jetting dynamics and correlation with the printing quality, Microfluidics and Nanofluidics 16, 493 (2013).

[24] R. Fardel, M. Nagel, F. N. T. Lippertesch, T. Lippert, and A. Wokaun, Laser-Induced Forward Transfer of Organic LED Building Blocks Studied by Time-Resolved Shadowgraphy, Physical Chemistry C 114, 5617 (2010).

[25] M. Domke, S. Rapp, M. Schmidt, and H. P. Huber, Ultra-fast movies of thin-film laser ablation, Applied Physics A 109, 409 (2012).

[26] D. Bartl, M. Ametowobla, F. Schmid, a. Letsch, M. Hafner, S. Nolte, and a. Tünnermann, Probing timescales during back side ablation of Molybdenum thin films with optical and electrical measurement techniques., Optics express 21, 16431 (2013).

[27] M. Feinaeugle, A. Alloncle, P. Delaporte, C. Sones, and R. Eason, Time-resolved shadowgraph imaging of femtosecond laser-induced forward transfer of solid materials, Applied Surface Science 258, 8475 (2012).

[28] S. A. Mathews, R. C. Y. Auyeung, H. Kim, N. a. Charipar, and A. Pique, High-speed video study of laser-induced forward transfer of silver nano-suspensions, Journal of Applied Physics 114, 064910 (2013).

[29] Y. Nakata and T. Okada, Time-resolved microscopic imaging of the laser-induced forward transfer process, Applied Physics A 69, 275 (1999).

[30] T. Sano, H. Yamada, T. Nakayama, and I. Miyamoto, Experimental investigation of laser induced forward transfer process of metal thin films, Applied Surface Science 186, 221 (2002). 
[31] A. B. Bullock and P. R. Bolton, Laser-induced back ablation of aluminum thin films using picosecond laser pulses, Journal of Applied Physics 85, 460 (1999).

[32] I. Zergioti, D. Papazoglou, A. Karaiskou, C. Fotakis, E. Gamaly, and A. Rode, A comparative schlieren imaging study between $n s$ and sub-ps laser forward transfer of $\mathrm{Cr}$, Applied Surface Science 208 - 209, 177 (2003).

[33] Z. Toth, B. Hopp, T. Szoerenyi, Z. Bor, E. A. Shakhno, and V. P. Veiko, in Proceedings of SPIE, edited by V. P. Veiko and T. Szoerenyi (SPIE, Munich, Germany, 1999), No. June, pp. 18-26.

[34] V. Veiko, E. Shakhno, V. Smirnov, A. Miaskovski, and G. Nikishin, Laser-induced film deposition by LIFT Physical mechanisms and applications, Laser and Particle Beams 24, 203 (2006).

[35] C. Unger, M. Grüne, L. Koch, J. Koch, and B. N. Chichkov, Time-resolved imaging of hydrogel printing via laser-induced forward transfer, Applied Physics A 103, 271 (2010).

[36] D. Ivanov and L. Zhigilei, Combined atomistic-continuum modeling of short-pulse laser melting and disintegration of metal films, Physical Review B 68, 064114 (2003).

[37] C. Mézel, L. Hallo, a. Souquet, J. Breil, D. Hébert, and F. Guillemot, Self-consistent modeling of jet formation process in the nanosecond laser pulse regime, Physics of Plasmas 16, 123112 (2009).

[38] D. S. Ivanov, A. I. Kuznetsov, V. P. Lipp, B. Rethfeld, B. N. Chichkov, M. E. Garcia, and W. Schulz, Short laser pulse nanostructuring of metals: direct comparison of molecular dynamics modeling and experiment, Applied Physics A 111, 675 (2013).

[39] T. C. Röder and J. R. Köhler, Physical model for the laser induced forward transfer process, Applied Physics Letters 100, 071603 (2012).

[40] A. I. Kuznetsov, C. Unger, J. Koch, and B. N. Chichkov, Laser-induced jet formation and droplet ejection from thin metal films, Applied Physics A 106, 479 (2012).

[41] J. M. Liu, Simple technique for measurements of pulsed Gaussian-beam spot sizes, Optics Letters 7, 196 (1982).

[42] S.-S.Wellershoff, J. Hohlfeld, J. Güdde, and E. Matthias, The role of electron-phonon coupling in femtosecond laser damage of metals, Applied Physics A 69, 99 (1999).

[43] M. V. Shugaev and N. M. Bulgakova, Thermodynamic and stress analysis of laser-induced forward transfer of metals, Applied Physics A 101, 103 (2010).

[44] B. Rethfeld, K. Sokolowski-Tinten, D. von der Linde, and S. Anisimov, Ultrafast thermal melting of laser-excited solids by homogeneous nucleation, Physical Review B 65, 092103 (2002).

[45] J. Hohlfeld, S.-S. Wellershoff, J. Güdde, U. Conrad, V. Jähnke, and E. Matthias, Electron and lattice dynamics following optical excitation of metals, Chemical Physics 251, 237 (2000).

[46] J. Garai, Physical model for vaporization, Fluid Phase Equilibria 283, 89 (2009).

[47] X. Zeng, X. Mao, R. Greif, and R. Russo, Experimental investigation of ablation efficiency and plasma expansion during femtosecond and nanosecond laser ablation of silicon, Applied Physics A 80, 237 (2004).

[48] R. Fardel, M. Nagel, F. Nuesch, T. Lippert, and A. Wokaun, Laser-Induced Forward Transfer of Organic LED Building Blocks Studied by Time-Resolved Shadowgraphy, The Journal of Physical Chemistry C 114, 5617 (2010).

[49] R. Pohl, C. Visser, G. Römer, C. Sun, and D. Lohse, in Proceedings of LAMP (LAMP, Niigata, Japan, 2013).

[50] F. Korte, J. Koch, and B. Chichkov, Formation of microbumps and nanojets on gold targets by femtosecond laser pulses, Applied Physics A 79, 879 (2004). 
[51] A. I. Kuznetsov, J. Koch, and B. N. Chichkov, Nanostructuring of thin gold films by femtosecond lasers, Applied Physics A 94, 221 (2009).

[52] D. A. Willis and V. Grosu, The effect of melting-induced volumetric expansion on initiation of laser-induced forward transfer, Applied Surface Science 253, 4759 (2007).

[53] R. Fardel, M. Nagel, F. Nüesch, T. Lippert, and A. Wokaun, Energy Balance in a Laser-Induced Forward Transfer Process Studied by Shadowgraphy, The Journal of Physical Chemistry C 113, 11628 (2009).

[54] F. J. Adrian, J. Bohandy, B. Kim, and A. Nette, A study of the mechanism of metal deposition by the laser-induced forward transfer process, Journal of Vacuum Science \& Technology B: Microelectronics and Nanometer Structures 5, 1490 (1987).

[55] B. Chichkov, C. Momma, and S. Nolte, Femtosecond, picosecond and nanosecond laser ablation of solids, Applied Physics A 115, 109 (1996). 


\section{6 \\ Towards 3D printing of pure metals by picosecond laser-induced forward transfer $* \dagger$}

Laser-induced forward transfer (LIFT) may in principle enable the three-dimensional printing of metals, but has not yet become a routine technology because hitherto it is too difficult to control it with sufficient accuracy. In LIFT, a pulsed laser is focused onto a thin metal donor film. This laser locally melts the film, resulting in the ejection of a micrometer-scale droplet, which can be deposited on a receiver substrate. By controlling and repeating the ejection at the same location, droplets are deposited at the same position and thus pillars are manufactured. In this work, we demonstrate the construction of copper pillars ( $2 \mathrm{~mm}$ long pillars with a diameter of $5 \mu \mathrm{m}$ ) and fill through-silicon vias using LIFT. The pillars have low porosity, are electrically conductive, and mechanically stable. We explain that the size and the velocity of the impacting droplet determine the solidification regime, and argue that the thermal properties of the receiver substrate are crucial for controlled deposition. Finally, we discuss future applications of this versatile 3D micromanufactuing technique.

\footnotetext{
*In preparation as: C.W. Visser,R. Pohl, et al.

${ }^{\dagger}$ Experiments (in part) experimental setup (in part), modeling, and first draft by CWV.
} 


\subsection{Introduction}

Great progress in additive manufacturing technologies now allows the fabrication of complex 3-dimensional structures. The mainstream technologies include printing, photopolymerization, powder bed fusion, sheet lamination or deposition, and directwrite technologies [1]. In particular the printing concept has gained momentum for rapid prototyping, since it provides for fast, low-cost, and contact-free deposition at room conditions and poses minimal disturbances to the receiver substrate (on which the material is deposited). For instance, deposition of wax, polymers [2], and even living cells [3] are routinely achieved. However, printing of metals remains challenging, since the melting temperature of most metals is similar to (components within) the printing nozzle. As yet, metal printing has been limited to low-melting point metals $[4,5]$ and metal-containing inks [6-8], which are generally not optimized in terms of material properties (e.g. strength, conductivity, and corrosion rate) and cost. Contact-free deposition of a wider range of metals may therefore enable novel applications including optimized micro-antennas [9], electrode deposition on rough or inclined surfaces, or filling through-silicon vias for connecting stacked 2D electronic circuits [10].

Laser-induced forward transfer (LIFT) is a direct-write method allowing for deposition of a wide range of metals [11-15] including chromium [16, 17], tungsten [16], gold [18, 19], nickel [18], and aluminium [20]. The concept is shown in figure 6.1 (a). A pulsed laser is focused on the material to be transferred, the "donor film", which is initially present on a transparent "carrier" substrate. The light is absorbed by the metal, resulting in a thermal stress wave or evaporation within a part of the film, which subsequently leads to the ejection of a metal micro-droplet. Printing is achieved by placing a receiver substrate in the droplet's line of flight,.

However, application of pure-metal LIFT for 3D direct-write has been limited to deposition of single metal droplets [21-23], lines [10, 24], and nanoparticles [25]. This is because two challenging requirements have to be simultaneously fulfilled for consistent deposition: (1) the landing position of a single droplet has to be limited to the previously deposited droplet's impact area, and (2) good adhesion between the droplets is required. For solid $[16,26]$ material transfer, which is mainly pursued to transfer intact parts of the donor film to the receiver, the adhesion is expected to be limited. Therefore, we aim for controlled deposition in the liquid phase. In this regime, for LIFT just above the ejection threshold fluence, a single spherical deposit is observed [21-23]. At intermediate ejection fluences, deposition of a torus-like shape is observed [21], which indicates that the droplet solidified in spread-out shape. At even higher fluences, a large amount of very small spherical droplets is observed $[17,27]$. However, as stacking spheres or torus-shaped structures unavoidably results 
in porosity and limited drop-to-drop contact after solidification, none of these shapes seems to be optimal for manufacturing high-density structures.

In this work however, a different, disk-shape solidification regime is observed, which seems to be ideal for droplet stacking. The disk-shape results in a larger contact area between the drops, and thereby could enhance the strength, homogeneity, and conductivity of the deposited structure. To characterize this regime, we visualize the droplet ejection dynamics, using real-time visualization at nanosecond time scales and micrometer resolution. Subsequently, we manufacture a micropillar by repeating the ejection events at the same spatial position, and define its features in detail. We extend these results to demonstrate the fabrication of extremely long pillars, the filling of holes, and the deposition of a conducting line with minimal defects. Finally, we discuss the relationship between the droplet impact velocity and its solidification characteristics, and we present a concise outlook for future 3D metal fabrication using LIFT.
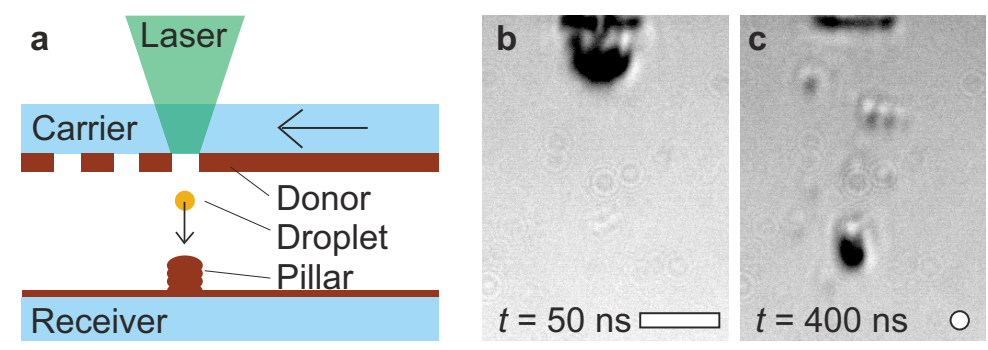

Figure 6.1: (a) LIFT setup. A laser pulse is focused onto a $200 \mathrm{~nm}$ thick copper donor layer, resulting in the ejection of a liquid copper droplet (shown as a yellow dot). By moving the carrier horizontally (shown by the arrow), repetitive ejections are produced at the same location. The impacting droplets solidify, resulting in the formation of a pillar on the receiver substrate. The receiver is coated with a copper film, to improve the adhesion of the droplet. Each ejection leaves behind a hole in the donor film: the "crater". Figure (b) and (c) show a single LIFT ejection for fluence $F=(4600 \pm 200) \mathrm{Jm}^{-2}, 50 \mathrm{~ns}$ and $400 \mathrm{~ns}$ after the laser is fired. In the top of both images, the crater is visible as a black line. In figure (b), the strongly deformed donor film is just detaching from the remaining substrate. In figure (c), the flying droplet is shown, which is trailed by several smaller satellite droplets. The droplet appears to be much smaller than in figure (a), as surface tension contracts the (initially hollow) droplet into a sphere with a final size as indicated by the white dot. Eventually, the droplet will reach a spherical shape and land on the receiver substrate (not shown). The scale bar indicates $10 \mu \mathrm{m}$. 


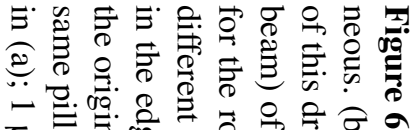

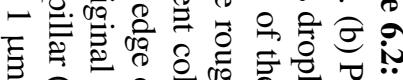

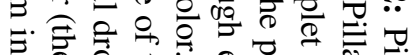

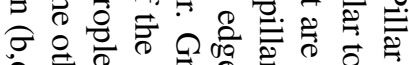

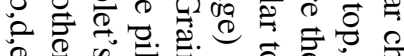

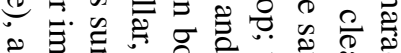

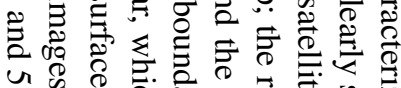

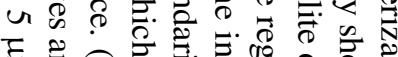

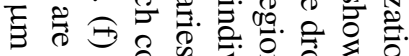

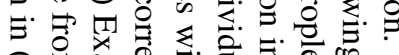

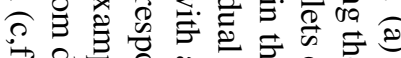
은훙

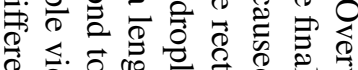

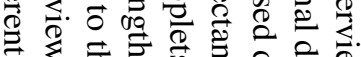

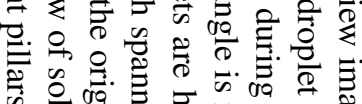

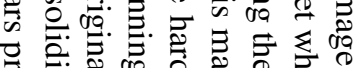

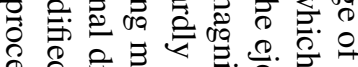

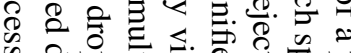

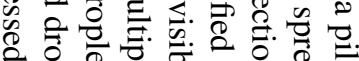

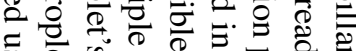

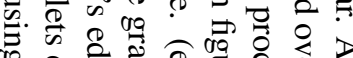
ㅁ. F 웡 क

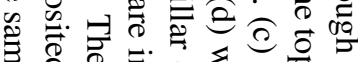
응

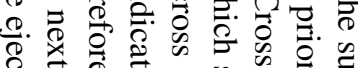
तै तै की

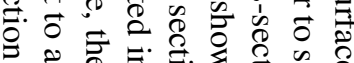

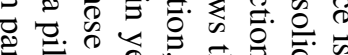
$\approx$ 政

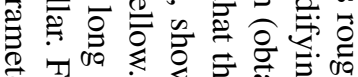
ब क्ष 붕 क्षे

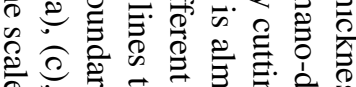

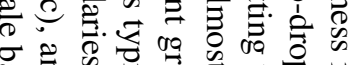

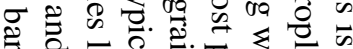

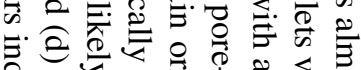

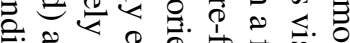

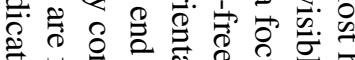

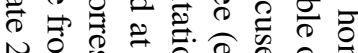

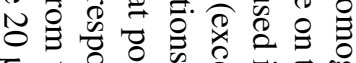

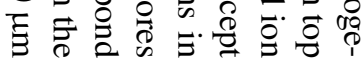

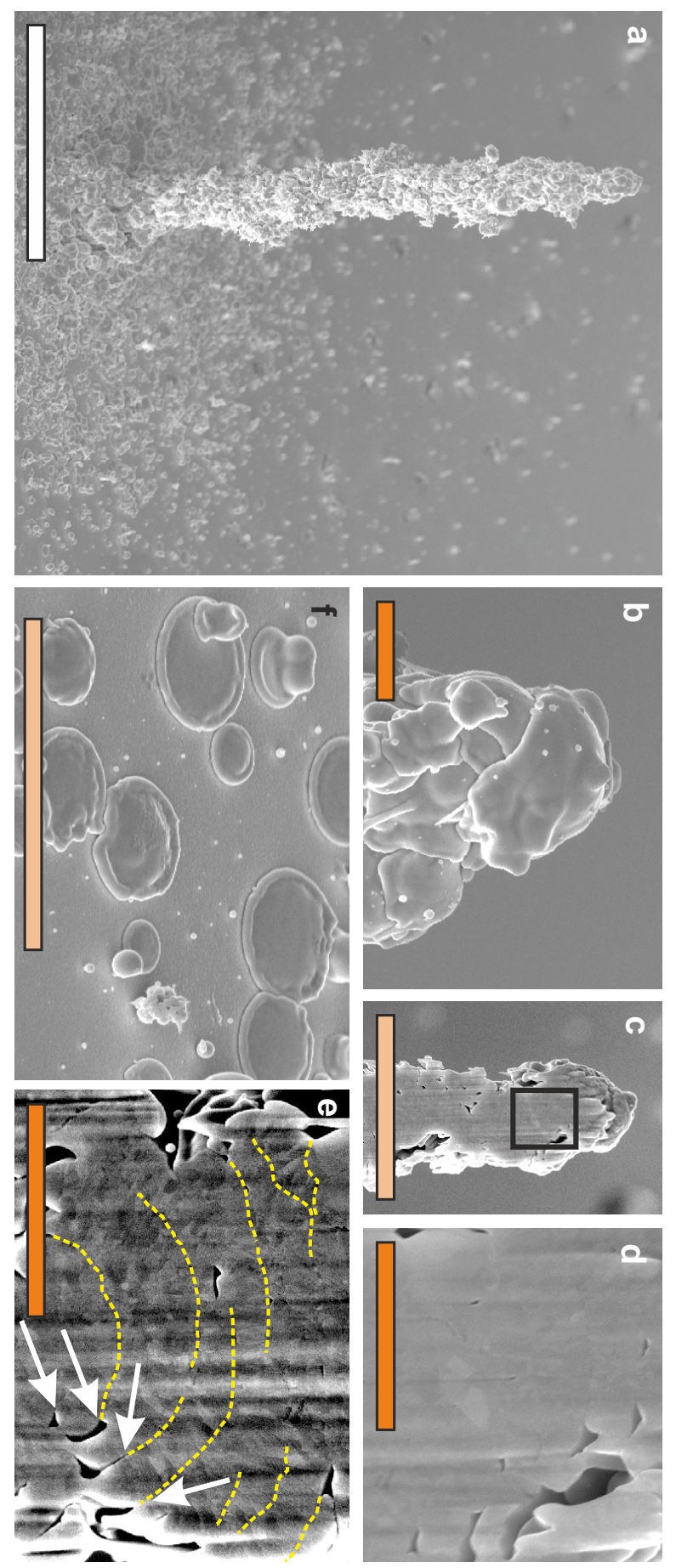




\subsection{Results}

\subsubsection{Pillar formation and characterization}

Laser-induced forward transfer of copper droplets is achieved using the setup depicted in figure 6.1 (a). In short, a laser with a pulse duration of $7 \mathrm{ps}$ and a wavelength of $515 \mathrm{~nm}$ is focused into a spot with a full-width, half-mean diameter of $10 \mu \mathrm{m}$, resulting in the melting and ejection of a $200 \mathrm{~nm}$ copper film. As shown in two snapshots of an ejection event, the ejected material initially maintains its sheetshape (figure 6.1 (b)), but strongly contracts (figure 6.1 (c)) and eventually reaches a spherical shape. The ejection velocity ranges from 30 to $200 \mathrm{~m} / \mathrm{s}$ [28]. The donor substrate is moved in horizontal direction using a motorized translation stage. Repeatedly shooting the laser then results in a series of ejection events at the same spatial position, which results in the formation of a pillar if a receiver substrate is inserted below the donor.

To our best knowledge, the copper pillar shown in figure 6.2 (a) is the first example of 3D-micromanufacturing using LIFT. The pillar has a height of around $80 \mu \mathrm{m}$ and a diameter of $5.3 \pm 0.7 \mu \mathrm{m}$. Despite its rough surface, the thickness is still reasonably homogeneous. The pillar top is magnified in figure 6.2 (b), showing the last-deposited drop which has spread out over the pillar top and subsequently solidified. Below this droplet, the edges of several solidified droplets are visible. To assess whether such round edges result in pores within the pillar, a pillar was cut lengthwise using a focused ion beam, and subsequently visualized using a SEM. As shown in figure 6.2 (c) and (d), the original droplets are hardly visible and only a few small pores are observed. Using a through-lens detector, the grains within the pillar become just visible. Grain sizes between $50 \mathrm{~nm}$ and $200 \mathrm{~nm}$ are typically observed and seem to constitute most of the pillar volume (our current imaging equipment does not allow for quantitative analysis). Furthermore, several long, horizontally-aligned grain boundaries are identified, as illustrated by the yellow dashed lines. The ends of these boundaries (at the outer surface of the pillar) frequently coincide with voids corresponding to the edge of solidified droplets. This footprint suggests that the impacting droplets do not melt the pillar surface during impact, but instead crystallize onto the previously deposited droplets. In principle, these horizontal interfaces could be weak or brittle. However, as the pillar can be bent significantly and homogeneously before plastic deformation occurs, the influence of these interfaces on the pillar characteristics seems to be limited.

The deposition dynamics and reproducibility are discussed following figure 6.3. The deposition of a $270 \mu \mathrm{m}$ high pillar is shown in figure 6.3 (a). In the final stage, the growth rate becomes constant as indicated by the line in figure 6.3 , and the pillar 
diameter is almost homogeneous. In the initial phase the pillar growth rate is suppressed, due to deviations in the ejection angle of the droplet resulting in a spread of the impact location. Therefore, the bottom $30 \mu \mathrm{m}$ of the pillar is slightly thicker. This thickening is easily prevented by reducing the donor-receiver distance by $\sim 50 \mu \mathrm{m}$, but could be beneficial if a strong pillar-receiver adhesion is required. The reproducibility of these pillars is high, and their positioning is easily controlled. Figure 6.3 (b) shows five pillars on the same receiver substrate, which were deposited in a few minutes by just moving the receiver and repeating a series of ejection events. Implementing an automated positioning stage for the receiver would readily allow for deposition of pillar-forests.

The electrical resistance of five pillars is displayed in figure 6.4. The resistance was measured at different stages in their deposition process (for details see the Methods section). The slope indicates the pillar resistance per unit length, which is normalized by the pillar area to provide the effective bulk resistance, $\rho_{E}$. For pillars processed in air at room conditions, $\rho_{E}=1.3 \cdot 10^{-6} \Omega \mathrm{m}$, which exceeds the resistance of bulk copper (which is $\rho_{E}=1.6 \cdot 10^{-8} \Omega \mathrm{m}$ ) by a factor of 80 . As oxidation of the metal droplets or the pillar tip during deposition was expected to cause this relatively high resistance, a next set of pillars was processed in an argon environment. As shown in figure 6.4 , this reduces the bulk resistivity to $\rho_{E}=8.4 \cdot 10^{-7} \Omega \mathrm{m}$, thus almost halves the resistance as compared to the air-processed pillars. However, the value of bulk copper is not obtained, presumably due to nanometer-scale inhomogenieties within the pillar (such as gas bubbles entrained during the deposition, or strongly aligned grain boundaries). Still, as both these values are several orders of magnitude below other metals which have been used for deposition of conductive wires (e.g. Gallium-Indium, for which $\rho_{E}=3 \cdot 10^{-3} \Omega \mathrm{m}$ [29]), the pillars printed here are expected to be suitable for out-of-plane electrode manufacturing.

The now-controlled pillar deposition concept can easily be extended. In figure 6.5 (a), a $860 \mu \mathrm{m}$ long pillar is deposited, by moving the substrate down during pillar deposition while maintaining a distance between the donor and the pillar tip in the range of $20 \mu \mathrm{m}$ to $200 \mu \mathrm{m}$. Higher pillars can easily be created, until at some height the position of the tip is no longer stable: small vibrations or light air flows immediately result in a "waving" motion of the tip. Therefore a wider distribution in the droplet impact location with respect to the tip is observed, resulting in a thicker tip. The longest homogeneous pillar we could deposit is $2 \mathrm{~mm}$ high and $5 \mu \mathrm{m}$ in diameter, providing a remarkable aspect ratio of 1:400. Next, LIFT can be applied to provide the out-of-plane interconnect between the different layers of stacked electronics called "through-silicon vias" (TSVs). Figure 6.5 (g) shows a cross section of such a TSV, in which the deposition extends to the $100 \mu \mathrm{m}$ deep bottom. A defect 

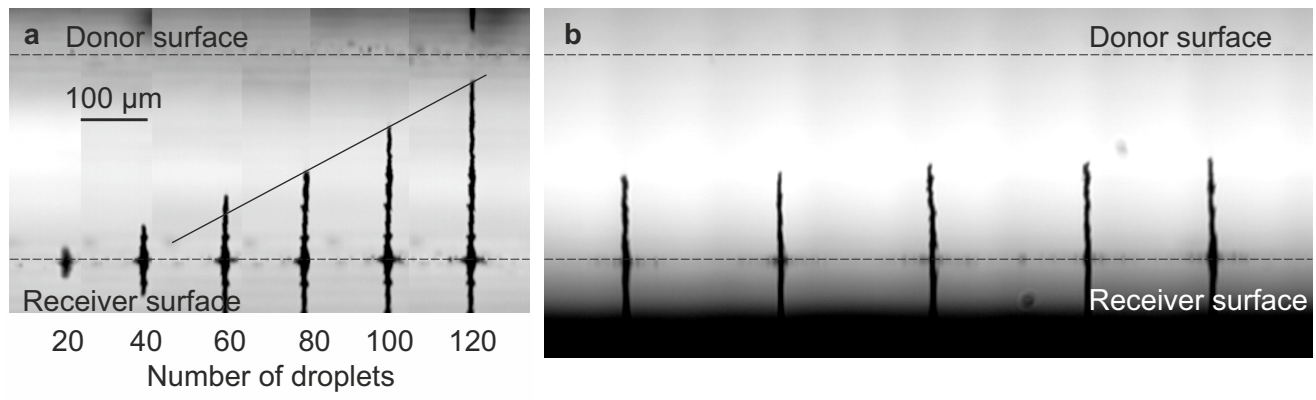

Figure 6.3: (a) Visualization of pillar formation. The numbers indicate the number of droplets constituting the pillar for each snapshot. The solid line indicates a constant pillar growth rate, which is observed if the pillar tip is sufficiently close to the donor surface and all droplets land on the tip. (b) Deposition of five pillars on the same receiver while using the same process parameters. In both sub-figures, the dashed lines indicate the (reflective) donor and receiver surfaces.

seems to be present close to the top, but since this region is closest to the donor substrate such defects can be repaired using LIFT (as yet, we have only one visualization of a filled hole, since their cutting without damaging the pillar proved highly challenging). As the top of the TSV is placed in close proximity to the carrier, unwanted debris (indicated by the arrow) is hardly observed. Finally, high aspect ratios can also be achieved in the horizontal plane: by moving the receiver substrate while ejecting repeatedly a conducting line is formed, shown in figure 6.5 (h). By controlling the receiver position also in the $x-y$ plane, truly $3 \mathrm{D}$ structures become in reach.

\subsection{Discussion}

The remarkable consistency of our pillars can be explained by the impact dynamics of the deposited droplets. To assess the time scales of impact, we consider a droplet with diameter $D_{0}$ impacting at velocity $V_{0}$ (see figure 6.6 (a)). Upon impact, the droplet spreads out over the surface and flattens, until a maximal spreading diameter $D_{\max }$ is reached (see figure $6.6(\mathrm{~b})$ ). The moment at which $D_{\max }$ is reached can be estimated by the inertial time scale $\tau_{I}=D_{0} / V_{0}$. Next, surface tension can have a profound influence on the spreading dynamics of these small droplets. In particular, a rim is formed at the edge of the droplet (see figure 6.6 (c)). This rim keeps growing also after the maximum spreading radius is reached. Eventually the droplet can even contract back into a sphere, as shown in figure 6.6 (d) (this only occurs for non- 


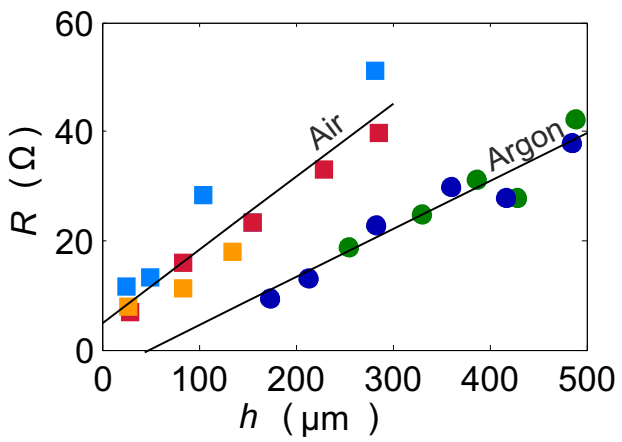

Figure 6.4: Electrical resistance of pillars processed in air (square markers) and argon (round markers) as a function of the pillar height. The different colors indicate different pillars. The slope of the fitted lines (in black) indicates the resistance per unit length. The corresponding bulk resistivity is 80 and 50 times higher than for bulk copper, for the air- and argon-processed pillars, respectively.

wetting surfaces, see note $\left.{ }^{\ddagger}\right)$. Contraction occurs on a capillary time scale $\tau_{\text {cap }}=$ $\sqrt{\rho D_{0}^{3} / \sigma}$, with $\sigma$ the surface tension and $\rho$ the drop's density (although the rim can already becomes thicker than the droplet's center thickness in an earlier stage).

The inertial- and capillary time scales are plotted in figure $6.6(\mathrm{e})$, based on the input parameters as derived in section 6.4 and our previously presented velocity model. First, we consider the inertial time scale $\tau_{I}$ and the capillary time scale $\tau_{c a p}$. For fluences just exceeding the ejection threshold fluence (bottom end of the lines), these time scales are almost equal, i.e. in this regime the formation of a significant rim in all phases of the spreading is expected. As visualized in the bottom SEM-image in figure 6.6, indeed a spherical droplet is observed just above the threshold fluence. Banks et al. [21] also deposited droplets in this rim-dominated regime, which also resulted in spherical droplets or strong rim formation as shown in figure 6.7. These observations are consitent with our hypothesis that a significant rim will be present in all stages of the droplet impact if $\tau_{I} \approx \tau_{\text {cap }}$. For intermediate fluences, the inertialand capillary-time scales separate as indicated by a green shading in figure 6.6 (e),

\footnotetext{
${ }^{\ddagger}$ An important remark here is that full contraction into a sphere (which can even bounce away from the surface) can occur only if the surface is non-wetting. This has been observed for hot metal droplets impacting on a standard glass substrate: due to heating of the glass by the droplet, the volatile elements evaporate and a vapor layer forms which prevents contact between the droplet and the glass [30]. In that case, the droplet does not or partly adhere to the substrate, which is why we coated our (glass) receiver surfaces with a $100 \mathrm{~nm}$ thick copper film (alternatively, heating of the glass is known to improve the droplet's adhesion [30]). The formation of a vapor film preventing contact between the droplet and the substrate is called the "Leidenfrost" phenomenon, for a review see ref. [31]
} 

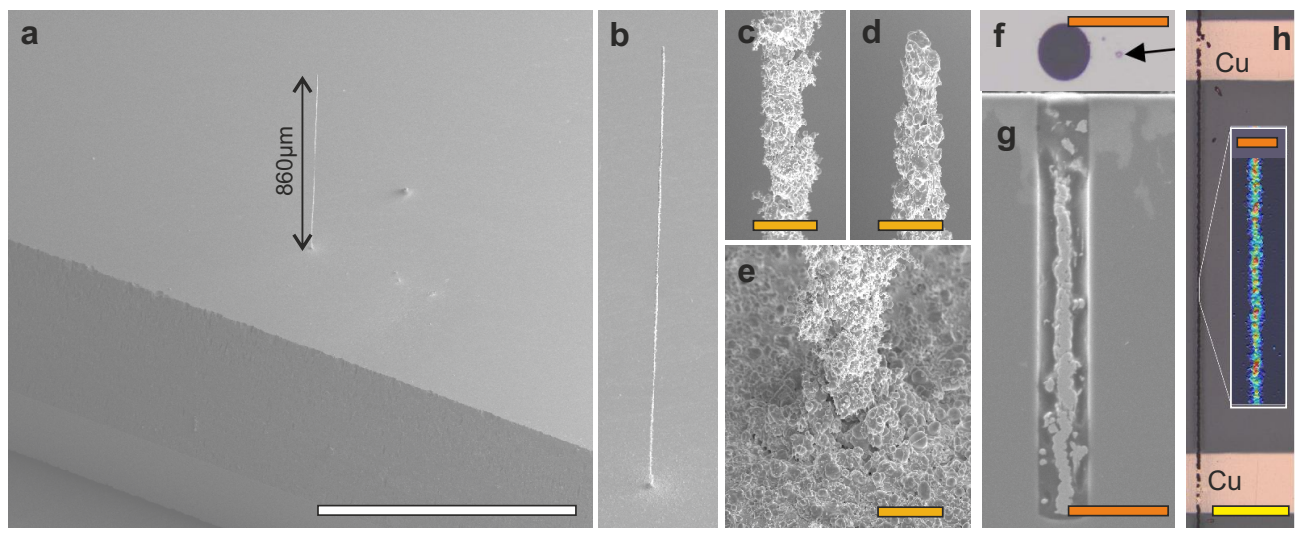

Figure 6.5: High-aspect ratio examples of copper LIFT. (a) $860 \mu \mathrm{m}$ long pillar close to the clearly visible edge of a microscope slide. (b) Close-up of the pillar. Zooming in further shows that the pillar has a virtually homogeneous thickness: the center (d) and top (e) are around $4 \mu \mathrm{m}$ thick whereas the bottom diameter (e) is around $6 \mu \mathrm{m}$, due to droplets landing next to the pillar. Figures (f) and (g) show a top view and a cross section of a filled TSV, respectively. The dark area next to the pillar (also visible from top) is a polymer for fixating the pillar, to avoid any harm due to grinding. The arrow in (f) indicates a defect, most likely caused by a droplet deposited next to the TSV. Image (g) shows that the pillar extends to the bottom of the hole. Finally, by moving the receiver substrate while depositing droplets, conductive lines can be fabricated as shown in figure (h).

and a disk-shaped solidification is expected and observed (the middle two SEM images). For high fluences, the impact of many very small droplets is observed (top SEM image in figure 6.6), which correseponds to the spray ejection regime reported previously $[21,28]$. Although we have been able to construct pillars in this regime, their growth rate is low and contamination is extremely high, due to the relatively wide range of ejection angles. Altogether, we hypothesize that disk-shape solidification can only be achieved if two constraints are fulfilled: First, the inertial time scale exceeds the capillary time scale; and second, the fluence value lies between the ejection threshold and the transition to spray formation.

Now that the control parameters for disk-shaped impact have been established, we focus on the cleanliness and overall control of the ejection. For our combination of donor film and laser properties, deposition of multiple droplets is almost always observed (see figure S1). This indicates poor location control which hampers pillar formation. However, in a narrow range of fluences, the deposition of multiple droplets on top of each other is observed, as also shown in figure 6.6 (second SEM image from top). This behavior is consistent and the location of impact seems to be 


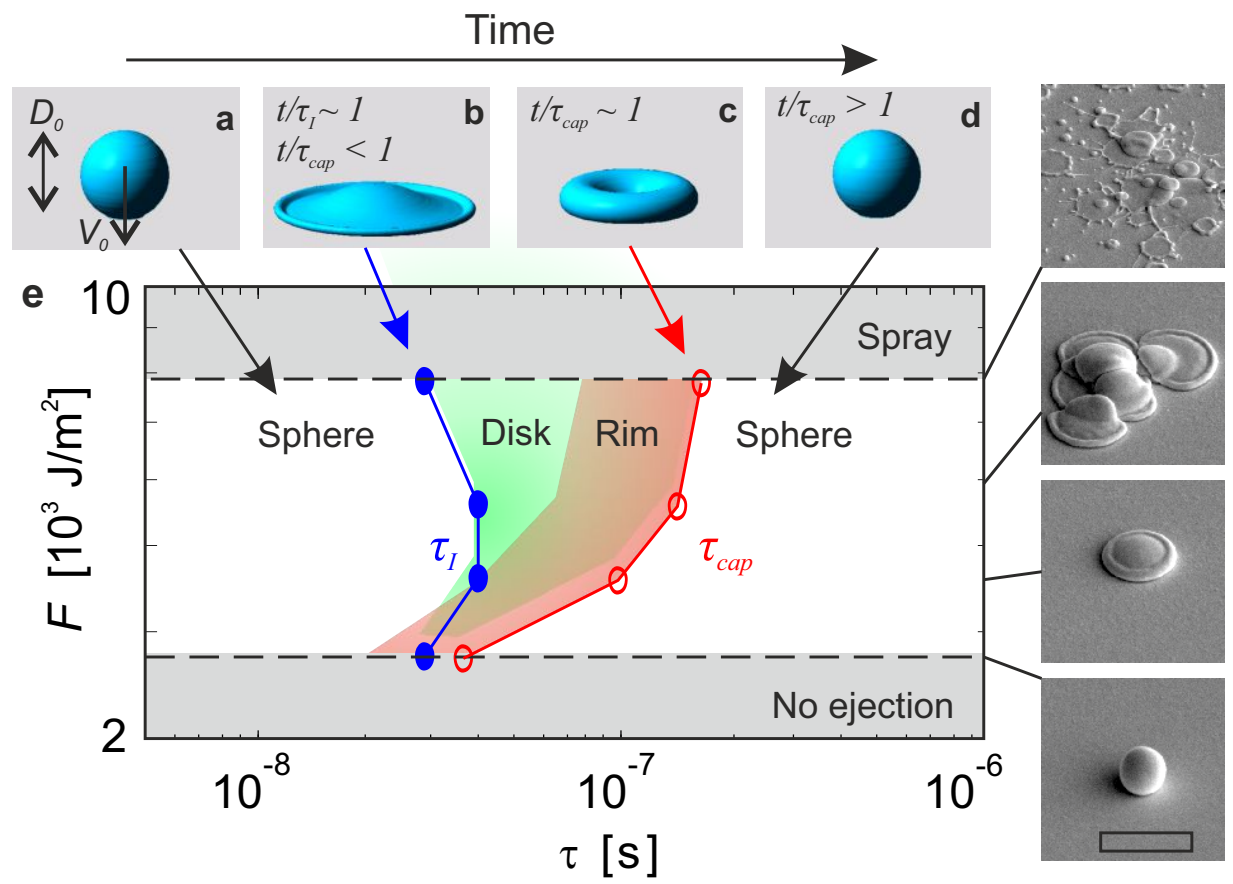

Figure 6.6: Droplet spreading dynamics and time scales of impact. Figures (a) to (d) show examples of droplet impact dynamics (surface not shown); images are a numerical simulations summarized in the Methods section. Figure (a) displays the droplet prior to impact; (b) illustrates the spreading, occurring on the inertial time scale $\tau_{I}$; (c) illustrates the retraction by the formation of a (growing) rim, occurring on the capillary time scale $\tau_{c a p}$; and (d) shows the retracted droplet. Figure (e) shows the inertial- and capillary time scales as solid lines (the markers represent calculations on measured data), for a copper donor film of $200 \mathrm{~nm}$ thick. In the center area, indicated by "disk", the droplet has an approximately flat shape. The ejection threshold fluence and the transition to the spray regime are measured as indicated by the dashed lines. For time scales $0.5 \tau_{\text {cap }}<\tau_{\text {cap }}$ the rim is very pronounced, therefore this "rim" area is shaded. The SEM-images (right) show solidified droplets deposited on a copper-coated surface, for fluences as indicated on the axis. The scale bar indicates $2 \mu \mathrm{m}$ and holds for all images. 


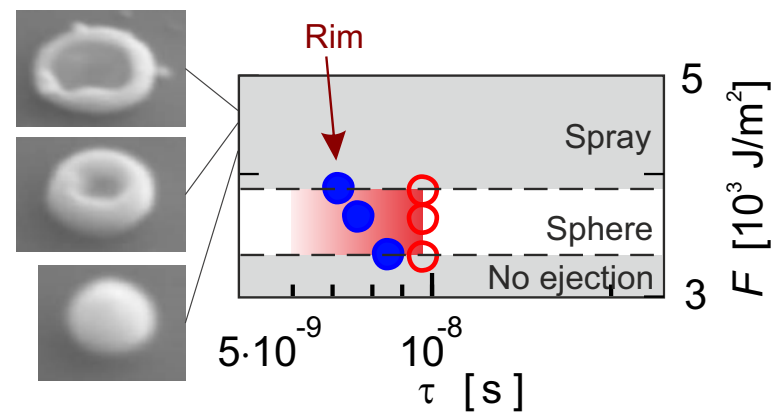

Figure 6.7: Illustration of droplet solidification exhibiting a pronounced rim. All raw data and all images are by Banks et al. [21]. The graph shows the inertial- and capillary time scales as solid lines (the markers represent calculations based on the measurements), for a Nickel donor film of $30 \mathrm{~nm}$ thick. The ejection threshold fluence and the transition to the spray regime are measured as indicated by the dashed lines. For time scales $0.5 \tau_{\text {cap }}<\tau_{\text {cap }}$ the rim is very pronounced, therefore this "rim" area is shaded (indicated by the arrow). The photographs show droplets solidified in the rim-regime.

robust, since all our pillars have been deposited in this regime. However, significant contamination is also observed as some droplets are not aligned with to the laser axis. In addition, deposition of small (and undesired) satellite droplets around the main droplet is observed. A plausible cause of both the larger off-axis droplets and the satellites is uncontrolled break-up of the ejected cap from the metal film, which we aim to study and optimize in future work. Alternatively, single-droplet ejection such as observed in ns-LIFT seems to be a very promising direction for future research, since the reported diameters and velocites would allow for disk-shaped deposition and satellite formation is hardly observed.

\subsection{Methods}

\subsubsection{LIFT ejection}

The experimental setup is shown in figure 6.1. The main laser is a Yb:YAG laser with a pulse duration $\tau_{L}=6.7 \mathrm{ps}$, a wavelength of $515 \mathrm{~nm}$, a Gaussian beam profile with quality factor $M^{2}<1.3$, and maximum peak fluence error of $200 \mathrm{~J} \mathrm{~m}^{-2}$. The beam is focused to a diameter $\left(1 / e^{2}\right)$ of $D_{L}=18 \mu \mathrm{m}$ at the carrier-donor interface, using an f-theta scan-lens with a $100 \mathrm{~mm}$ focal length. The fluences in this article are the peak fluences on the laser symmetry axis. A $d=(200 \pm 20) \mathrm{nm}$ copper donor layer 
is sputtered onto a $1 \mathrm{~mm}$ thick glass carrier. A clean glass microscope slide is used as a receiver substrate.

\subsubsection{Real-time visualization}

Images are recorded using a microscope with a $50 \times$ long-distance objective and a dual-shot CCD camera (PCO Sensicam qe, PCO, Germany). A dual-cavity Nd:YAG laser (with a pulse duration of $6 \mathrm{~ns}$ ) is used for bright-field flash illumination. To prevent coherence, a high-efficiency diffuser is placed in between the laser and the camera. The illumination laser and the camera are synchronized to the main laser by a BNC 575 pulse-delay generator, which in turn is triggered by the output of a photodiode connected to the LIFT laser beam path. This provides two flash-illuminated frames of each ejection with a minimum frame-to-frame time of $350 \mathrm{~ns}$, which were contrast-enhanced for optimal visualization.

\subsubsection{Pillar resistivity measurements}

The electrical properties of the pillars are measured by connecting electrodes to the donor and receiver substrates, and gently pressing the donor onto the tip of the pillar. In this way, the circuit is closed and the resistance is measured. The resistance consists of the contact resistance, $R_{c}$, and the resistance of the pillar itself. By measuring this resistance during the deposition process, i.e. for pillars of increasing length, increasing values of the resistance are obtained. A linear fit of the $R(h)$ curve provides the (constant) contact resistance $R_{c}$ as its offset, and the slope provides the pillar resistance per unit length.

\subsubsection{Derivation of input parameters for time scale calculations}

The input variables $D_{0}, D_{\max }$, and $V_{0}$ are required for calculating the time scales for spreading, contraction, and solidification. For the droplet diameter, $D_{0}$, we assume that the droplet has a spherical shape and a volume equal to the volume ejected from the donor layer (by measuring the crater size and depth). The droplet impact velocity $V_{0}$ is assumed to be equal to the ejection velocity, for which we recently derived and validated a model. For the data by Banks et al. [21], the intial droplet radius was derived from estimating the droplet volume post-solidification, and the velocity of their ejection was derived using our recent model [28]. Air drag, which results in an estimated velocity decrease of $\sim 0.2 V_{0}$ for the first $200 \mu \mathrm{m}$ of travel, is ignored. The maximal spreading diameter of the droplet is estimated using the model of Roisman (2009), as it includes both the inertial spreading dynamics and the rim formation, and 
is known to well describe experimental data. The maximal spreading diameter is thus provided as

$$
\frac{D_{\max }}{D_{0}}=0.87 \mathrm{Re}^{1 / 5}-0.4 \mathrm{Re}^{2 / 5} \mathrm{We}^{-1 / 2}
$$

Here, $\operatorname{Re}=\rho D_{0} V_{0} / v$ is the Reynolds number, which denotes the ratio between inertial energy to viscous dissipation. The temperature-dependent viscosity $v(T)$ is calculated according to ref. [32]. We $=\rho D_{0} V_{0}^{2} / \sigma$ is the Weber number, representing the ratio between inertial energy and surface energy. The model is valid for Re $\gg 1$ and $\mathrm{We} \gg 1$, which is the case for the droplets studied here.

The input parameters are plotted in figures 6.8 (d) and(e), both for our ejections and ref. [21] (in which a $30 \mathrm{~nm}$ chromium donor film is used). The impact velocity is plotted as a function of the fluence in figure 6.8 (d), which ranges from 30 to 100 $\mathrm{ms}^{-1}$. Also the threshold fluences for the cap ejection regime, in which stable droplet ejection is observed, are indicated. In particular, below a lower fluence threshold, no ejection is observed, and beyond a certain fluence a transition occurs to the "spray" regime, in which many similar-sized droplets are ejected. Our cap ejection regime has a wide fluence range compared to ref. [21], mainly because the transition to the spray regime occurs at a higher fluence for thicker donor films. Figure 6.8e shows the measured initial diameter $D_{0}$ (for the results by Banks et al. [21], photographs of the deposits are used to obtain $D_{0}$ ) and the maximal spreading radius as calculated from equation (6.1). In all cases, $D_{\max }>2 D_{0}$, but lower values are observed (open markers) as equation (6.1) does not include solidification.

\subsubsection{Numerical methods}

Simulations were done using the open-source flow solver Gerris [33], which numerically solves the incompressible Navier-Stokes equation on a non-uniform flowadaptive grid. The size of the (squared) simulation domain is 3 droplet diameters, with outflow boundary conditions on the top and left edge, no-flow conditions on the bottom edge and symmetry conditions on the right edge (which represent the z-axis along which the droplet approaches the surface). A further description and an extensive validation against experiments are provided in ref. [34].

\section{References}

[1] I. Gibson, D. W. Rosen, and B. Stucker, Additive Manufacturing Technologies (Springer US, Boston, MA, 2010), No. 12. 
a
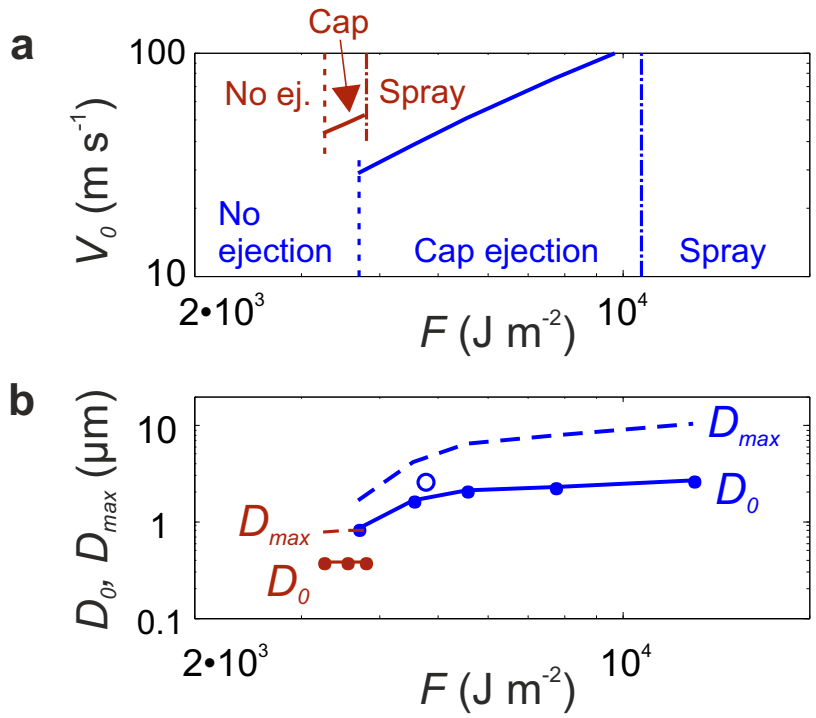

Figure 6.8: Figure (a), impact velocity encountered in LIFT, for our system (in blue, as validated in ref. [28]) and calculated based on the fluences used in Banks et al. (2006) [21]. The dashed lines indicate the experimentally observed transitions between no ejection, the capejection regime (in which one main droplet is ejected, usually accompanied by some satellite droplets), and the spray-ejection regime (in which many small droplets are ejected). In figure (b), the initial diameter $D_{0}$ and the maximal spreading diameter $D_{\max }$ are shown. $D_{\max }$ is calculated using equation (6.1), which does not include solidification. A somewhat smaller $D_{\max }$ is observed, as indicated by the open marker, since the droplets generally solidify before or after reaching $D_{\max }$. 
[2] B.-J. deGans, P. Duineveld, and U. Schubert, Inkjet Printing of Polymers: State of the Art and Future Developments, Advanced Materials 16, 203 (2004).

[3] S. Tasoglu and U. Demirci, Bioprinting for stem cell research., Trends in Biotechnology 31, 10 (2013).

[4] K. Yamaguchi, Generation of 3-dimensional microstructure by metal jet, Microsystem Technologies 9, 215 (2003).

[5] C. Ladd, J.-H. So, J. Muth, and M. D. Dickey, 3D printing of free standing liquid metal microstructures., Advanced materials (Deerfield Beach, Fla.) 25, 5081 (2013).

[6] J. Perelaer, P. J. Smith, D. Mager, D. Soltman, S. K. Volkman, V. Subramanian, J. G. Korvink, and U. S. Schubert, Printed electronics: the challenges involved in printing devices, interconnects, and contacts based on inorganic materials, Journal of Materials Chemistry 20, 8446 (2010).

[7] B. K. Park, D. Kim, S. Jeong, J. Moon, and J. S. Kim, Direct writing of copper conductive patterns by ink-jet printing, Thin Solid Films 515, 7706 (2007).

[8] S. H. Ko, J. Chung, N. Hotz, K. H. Nam, and C. P. Grigoropoulos, Metal nanoparticle direct inkjet printing for low-temperature $3 D$ micro metal structure fabrication, Journal of Micromechanics and Microengineering 20, 125010 (2010).

[9] J. J. Adams, E. B. Duoss, T. F. Malkowski, M. J. Motala, B. Y. Ahn, R. G. Nuzzo, J. T. Bernhard, and J. a. Lewis, Conformal printing of electrically small antennas on three-dimensional surfaces., Advanced materials (Deerfield Beach, Fla.) 23, 1335 (2011).

[10] G. Oosterhuis, A. Prenen, and A. Huis in 't Veld, Laser Induced Forward Transfer of Interconnects for $3 D$ Integration, ECS transactions 41, 81 (2012).

[11] V. Schultze and M. Wagner, Blow-off of aluminium films, Applied Physics A 53, 241 (1991).

[12] I. Zergioti, S. Mailis, N. Vainos, C. Fotakis, S. Chen, and C. Grigoropoulos, Microdeposition of metals by femtosecond excimer laser, Applied Surface Science 127-129, 601 (1998).

[13] P. Mogyorósi, T. Szörényi, K. Bali, Z. Tóth, and I. Hevesi, Pulsed laser ablative deposition of thin metal films, Applied surface science 36, 157 (1989).

[14] H. Esrom, J.-Y. Zhang, U. Kogelschatz, and A. J. Pedraza, New approach of a laser-induced forward transfer for deposition of patterned thin metal films, Applied Surface Science 86, 202 (1995).

[15] Z. Tóth, T. Szörényi, and A. Tóth, Ar+laser-induced forward transfer (LIFT): a novel method for micrometer-size surface patterning, Applied surface science 69, 317 (1993).

[16] Z. Tóth, B. Hopp, T. Szoerenyi, Z. Bor, E. A. Shakhno, and V. P. Veiko, in Proceedings of SPIE, edited by V. P. Veiko and T. Szoerenyi (SPIE, Munich, Germany, 1999), No. June, pp. 18-26.

[17] I. Zergioti, D. Papazoglou, a. Karaiskou, C. Fotakis, E. Gamaly, and a. Rode, A comparative Schlieren imaging study between ns and sub-ps laser forward transfer of $\mathrm{Cr}$, Applied Surface Science 208-209, 177 (2003).

[18] T. Sano, H. Yamada, T. Nakayama, and I. Miyamoto, Experimental investigation of laser induced forward transfer process of metal thin films, Applied surface science 186, 221 (2002).

[19] Y. Nakata and T. Okada, Time-resolved microscopic imaging of the laser-induced forward transfer process, Applied Physics A 278, 275 (1999).

[20] A. B. Bullock and P. R. Bolton, Laser-induced back ablation of aluminum thin films using picosecond laser pulses, Journal of Applied Physics 85, 460 (1999).

[21] D. P. Banks, C. Grivas, J. D. Mills, R. W. Eason, and I. Zergioti, Nanodroplets deposited in microarrays by femtosecond Ti:sapphire laser-induced forward transfer, Applied Physics Letters 
89, 193107 (2006).

[22] L. Yang, C.-y. Wang, X.-c. Ni, Z.-j. Wang, W. Jia, and L. Chai, Microdroplet deposition of copper film by femtosecond laser-induced forward transfer, Applied Physics Letters 89, 161110 (2006).

[23] A. I. Kuznetsov, C. Unger, J. Koch, and B. N. Chichkov, Laser-induced jet formation and droplet ejection from thin metal films, Applied Physics A 106, 479 (2012).

[24] C. Germain, L. Charron, L. Lilge, and Y. Y. Tsui, Electrodes for microfluidic devices produced by laser induced forward transfer, Applied Surface Science 253, 8328 (2007).

[25] C. M. Othon, A. Laracuente, H. Ladouceur, and B. R. Ringeisen, Sub-micron parallel laser directwrite, Applied Surface Science 255, 3407 (2008).

[26] H. Yamada, T. Sano, T. Nakayama, and I. Miyamoto, Optimization of laser-induced forward transfer process of metal thin films, Applied Surface science 198, 411 (2002).

[27] T. V. Kononenko, P. Alloncle, V. I. Konov, and M. Sentis, Shadowgraphic imaging of laser transfer driven by metal film blistering, Applied Physics A 102, 49 (2010).

[28] R. Pohl, C. W. Visser, G. R. B. E. Römer, D. Lohse, C. Sun, and A. J. Huis in 't Veld (unpublished).

[29] D. Zrnic and D. Swatik, On the resistivity and surface tension of the eutectic alloy of gallium and indium, Journal of the less common metals 18, 67 (1969).

[30] A. McDonald, M. Lamontagne, C. Moreau, and S. Chandra, Impact of plasma-sprayed metal particles on hot and cold glass surfaces, Thin Solid Films 514, 212 (2006).

[31] D. Quéré, Leidenfrost Dynamics, Annual Review of Fluid Mechanics 45, 197 (2013).

[32] G. Kaptay, A unified equation for the viscosity of pure liquid metals, Zeitschrift für Metallkunde 7, 1 (2005).

[33] S. Popinet, An accurate adaptive solver for surface-tension-driven interfacial flows, Journal of Computational Physics 228, 5838 (2009).

[34] C. W. Visser, P. E. Frommhold, S. Wildeman, R. Mettin, D. Lohse, and C. Sun (unpublished). 


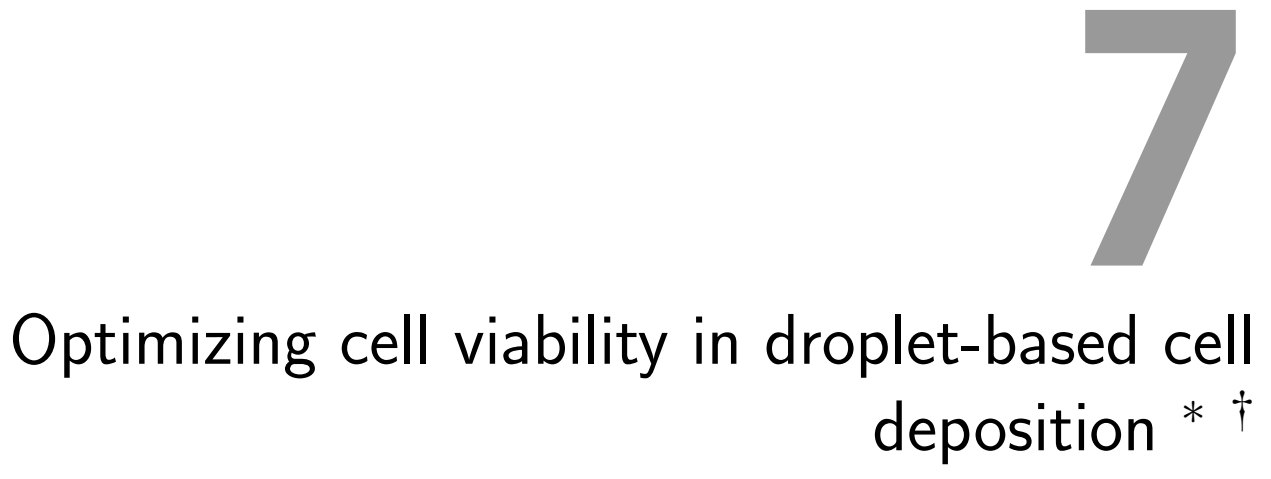

Biofabrication commonly involves the use of liquid droplets to transport cells to the printed structure. However, the viability of the cells after impact is poorly controlled and understood, hampering applications including cell spraying, inkjet bioprinting, and laser-assisted cell transfer. Here, we present an analytical model describing the cell viability after impact as a function of the cell-surrounding droplet characteristics. The model connects (1) the cell survival as a function of cell membrane elongation, (2) the membrane elongation as a function of the cell-containing droplet size and velocity, and (3) the substrate properties. The model is validated by cell viability measurements in cell spraying, which is a novel method for biofabrication and used for the treatment of burn wounds. The results allow for rational optimization of any droplet-based cell deposition technology, and we include practical suggestions to improve the cell viability in cell spraying.

${ }^{*}$ Submitted as: J. Hendriks, C.W. Visser, S. Henke, J. Leijten, D.B.F. Saris, D. Lohse, C. Sun, M. Karperien

${ }^{\dagger}$ Research design, measurements, cell survival model, data analysis, and first draft by CWV (all in part). 


\subsection{Introduction}

Droplet-based cell deposition is receiving increasing attention as a tool to construct or fill a variety of biological tissues. Striking examples are the cell spray treatment of burns [1,2] or ulcers [3], which provide faster and improved healing and are currently introduced in clinical practice. With one successful application in place, we seek to expand to other clinical areas including laparoscopic, endoscopic, and arthroscopic procedures [4]. This opens the possibility for minimally invasive cell therapy for tissue regeneration. A second example is the field of biofabrication, in which functional tissue replacements are fabricated in a laboratory and used for curing non-functional tissues [5-7]. In current biofabrication technologies including ink-jet bioprinting [810], laser-induced forward transfer [11], valve-based bioprinting [12, 13], and cell spraying [2, 14-17], the cell transport from the initial cell suspension, called "bioink", to the manufactured tissue is achieved by liquid droplet ejection and deposition. Although these technologies allow for high-viability cell deposition, limited throughput, limited precision, and poorly optimized cell-containing bio-inks are major obstacles in the controlled deposition of cells, such as required for the fabrication of functional tissues [5, 7].

To solve these issues and thereby optimize droplet-based cell deposition, knowledge of the cell viability as a function of the cell-containing droplet size and impact velocity is crucial. Ideally, single, highly reproducible impacts of droplets containing a single cell would be monitored for a large range of the impact parameters (droplet size, velocity, and material properties). Drop-on-demand systems provide such highly reproducible droplets, but usually the impact parameter space is relatively narrow for the cell-containing liquids used [18-23]. Therefore, to study postimpact cell viability, we use cell spray deposition, which allows for a much larger range of impact parameters. The substantial influence of the spray parameters on post-impact cell viability $[2,15-17,24]$ suggests that cell viability can be controlled, providing a model system to assess cell survival after impact. Additionally, the shear stress exerted on the cell within the spray nozzle is much lower than the shear stress during impact, which allows for assessment of the impact process alone (for other technologies this is not the case, as explained in supplementary section I).

The current work aims to understand the influence of the droplet impact on cell viability, which is applicable both to drop-on-demand and spray deposition technologies. We introduce a model describing the cell viability as a function of the cellcontaining droplet size, the viscosity, and the impact velocity (section 7.2.1). The model is validated by cell spray experiments, following a two-step approach. First, the droplet size and impact velocity are measured and used to obtain model predictions as described in section 7.2.2. Subsequently, in section 7.2.3, the cell viability 
after spraying is measured as a function of the air pressure, the liquid viscosity, the nozzle-substrate distance, and the substrate stiffness. The model is shown to accurately describe the viability measurements as a function of the input parameters. These results provide a powerful tool to rationally evaluate and improve clinical spray treatments and tissue engineering applications, as discussed in section 7.3.

\subsection{Results}

\subsubsection{Cell viability model}

In cell spraying, cell damage is primarily expected during impact of the cell-containing droplets (see supplementary section I). In particular, impact generally results in cell deformation and elongation of the cell membrane [25], as illustrated in figure 7.1(c) and (d). For an increase of the cell membrane area up to $\sim 5 \%$, the membrane is stretched, but remains intact. However, for larger extensions, rupture can be observed [26]. As rupture generally results in cell death, the probability of survival $\eta$ is modeled as a function of the relative cell membrane area $\gamma$ (compared to the undisturbed case) according to ref. [27]:

$$
\begin{array}{ccc}
\eta(\gamma)=1 \text { for } & \gamma<\gamma_{c r}-\Delta \gamma \\
\eta(\gamma)=\frac{1}{2}-\frac{\gamma-\gamma_{c r}}{2 \Delta \gamma} \text { for } & \gamma_{c r}-\Delta \gamma<\gamma<\gamma_{c r}+\Delta \gamma \\
\eta(\gamma)=0 \text { for } & \gamma>\gamma_{c r}+\Delta \gamma
\end{array}
$$

with $\gamma_{c r}=1.5$ the critical membrane expansion as quantitatively provided in ref. [27], and $2 \Delta \gamma=1$ the range of surface expansion in which the cells partly survive. Key model conditions include an elastic cell response to stresses (which is fulfilled for shear rates $\tau \lesssim 10^{9} \mathrm{~s}^{-1}$ [26]) and negligible lipid membrane replenishment during deformation (fulfilled for $\tau \gtrsim 10^{-3} \mathrm{~s}^{-1}$ [28]), which are met in the current work.

To obtain the relative cell membrane area $\gamma$, first a "clean" cell impact on a hard substrate is considered. The cell is described as a spherical liquid droplet with diameter $D_{c}$, velocity $V_{c}$, viscosity $\mu_{c}$, density $\rho_{c}$ and surface tension $\sigma_{c}$. The maximal spreading diameter reached during impact $D_{c, \max , 0}$ is then calculated as a function of the cell Weber number, which describes the ratio between kinetic energy and surface energy [29, 30]:

$$
\begin{array}{r}
D_{c, \text { max }, 0} / D_{c}=1.25 \text { for } \quad \mathrm{We}_{c}<5 \\
D_{c, \text { max }, 0} / D_{c} \sim \mathrm{We}_{c}^{1 / 4} \text { for } \quad \mathrm{We}_{c} \gtrsim 5,
\end{array}
$$



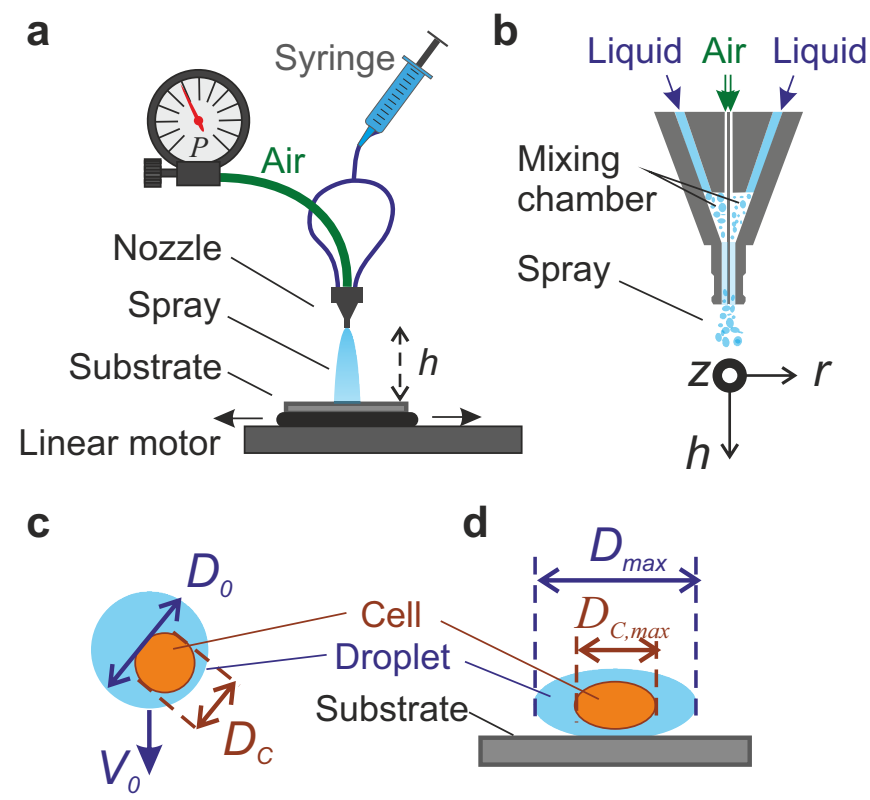

Figure 7.1: Overview of the experimental method and parameter definition. A two-phase spray nozzle is used to generate the spray, and placed at a distance $h$ from the surface. The cell-containing liquid (with viscosity $\mu$ ) is delivered to the nozzle using a syringe pump (not shown). Air at controlled pressure $P$ is applied to the nozzle gas inlet. The impact substrate is moved under the spray (indicated by horizontal arrows) using a linear motor, ensuring clean and homogeneous impact. Generally, a clean glass substrate is used, but gelatin-water mixtures (with gelatin weight fractions $C_{g}$ ) are used to assess the influence of the surface stiffness. (b) Cross-section of the nozzle, illustrating the air and liquid flows, and the coordinate system used. Figures (c) and (d) illustrate key variables describing the cell-containing droplets in air (c) and during impact (d). 
with $\mathrm{We}_{c}=\rho_{c} D_{c} V_{c}^{2} / \sigma_{c}$. The cell shape is defined by assuming cell deformation into an oblate spheroid and volume conservation. At the instant of reaching its maximal extension, its height equals $h_{0}=D_{c}^{3} / D_{c, \max , 0}^{2}$. This provides the deformation $M_{0}$ for an impacting cell, which is defined according to ref. [25]:

$$
M_{0}=\frac{D_{c, \max , 0}-h_{0}}{D_{c, \max , 0}+h_{0}}=\frac{D_{c, \max , 0}^{3}-D_{c}^{3}}{D_{c, \max , 0}^{3}+D_{c}^{3}},
$$

i.e. $M_{0}=0$ for a sphere, and $M_{0}=1$ for a plane.

The potentially large influence of the surrounding droplet on the cell's deformation was modeled numerically by Tasoglu et al. [25]. Re-interpreting their results provides a quantitative expression capturing the cell deformation $M$ as a function of $M_{0}$, the surrounding droplet's diameter $D_{0}$, and its viscosity $\mu_{0}$ (for details see supplementary section III):

$$
M=C_{0} M_{0} \cdot e^{-0.26 D_{0} / D_{c}}\left(\frac{\mu_{c}}{\mu_{0}}\right)^{-0.56}
$$

with $C_{0}$ a fitting parameter (which is set to $C_{0}=5$, as discussed in supplementary section III). Subsequently, $M$ is translated into the maximal spreading diameter of the (oblate-spheroid) cell as $\frac{D_{c, m a x}}{D_{c}}=\left(\frac{1+M}{1-M}\right)^{1 / 3}$, which is used below to calculate the cell surface area. Since this occasionally results in a cell diameter exceeding the droplet's diameter, we additionally implement the condition $D_{c, \max }=\min \left(D_{c, \max , 0}, D_{\max }\right)$. The surface area $S$ of an oblate spheroid (the assumed shape of a single trypsinized cell) can be calculated as:

$$
A=\frac{\pi D_{c, \max }^{2}}{2}\left(1+\frac{1-e^{2}}{e} \tanh ^{-1} e\right)
$$

with $e^{2}=1-h^{2} / D_{c, \max }^{2}$. The relative surface area is given by $\gamma=A / \pi D_{c}^{2}$, which completes the system.

Finally, the model is extended to account for the stiffness of the impact substrate. We assume impact on a liquid pool (with material properties equal to the droplet) as a soft surface limit. A droplet with diameter $D_{0}$ and velocity $V_{0}$ impacting on such a pool is (in first approximation) described by the impact of a droplet with diameter $2 D_{0}$ and velocity $0.5 V_{0}$ on a hard substrate [31]. As soft-surface droplet impact is not adequately understood even for basic model systems [32], we propose an effective droplet diameter and velocity as:

$$
D_{\text {eff }}=\frac{2 D_{0}}{1+S} \quad \text { and } \quad V_{\text {eff }}=\frac{V_{0}(1+S)}{2}
$$


with $S$ an arbitrary stiffness parameter ranging from $S=0$ for liquid surfaces to $S=1$ for hard surfaces. A gelatin-water mixture is used to generate a substrate stiffness range corresponding to a large variety of natural tissues [33, 34]. In the high-shear regime associated to fast micro-droplet impact the viscoelastic properties of these substrates cannot be measured by any standard viscometer. Therefore, the gelatin mass fraction $C_{g}$ is used to define the stiffness: $S=C_{1} C_{g}$, with $C_{1}$ a fitting constant. Using $C_{1}=5$ provides reasonable agreement between the model and our soft-surface impact measurements. For $C_{g}>1 / C_{1}$ we define $S=1$, which implies an effectively stiff surface for $C_{g}>0.2$.

Figure 7.2 shows example model results. The viability probability of individual cells is shown as a function of the impact velocity, for different sizes of the surrounding droplet (figure 7.2(a)), and different relative viscosities (figure 7.2(b)). At low velocities, the cell viability is only weakly dependent on the impact parameters since a small and constant cell deformation is assumed for low Weber numbers (We $<5$ ). For increasing velocities (corresponding to $\mathrm{We}>5$ ) a decrease in cell viability is observed. In this regime, the size of the surrounding droplet and its viscosity strongly affect cell viability. Larger surrounding droplets provide stronger cushioning and thereby increase the viability (figure 7.2(a)). Increasing the droplet viscosity negatively influences the cell viability, since for $\mu_{c} \ll \mu_{0}$ the droplet will flow around the (relatively stiff) cell, whereas for $\mu_{c}<\mu_{0}$ the cell flows to dampen the (relatively stiff) droplet's impact, resulting in significant cell deformation and decreased viability. Finally, softer substrates provide increased cushioning as shown by the color gradient in figure 7.2(a). Here, the surface deforms such that the deformation of the droplet is reduced. Consequently, cell deformation is suppressed and a higher viability is expected. In conclusion, optimal cell viability is expected for slow, large, and low-viscosity cell-containing droplets impacting onto a soft surface.

\subsubsection{Spray characterization}

To obtain cell viability predictions from the model, the droplet size- and velocity are required. We obtain these parameters according to figure 7.3. First, the droplets are visualized as shown in figure 7.3(a) and (b). Automated image analysis then provides the droplet diameter $D_{0}$ and velocity $V_{0}=\Delta h / \Delta t$, as illustrated in figure 7.3(c) and (d). For any spray experiment, a wide range of droplet sizes and velocities is observed. A representative sample of droplet sizes and velocities is indicated by the black markers in figure 7.3(e), which also contains indicative cell viability contours (similar graphs for different spraying parameters are included in supplementary section II). For each droplet, the cell viability is calculated using our model. Now the droplets are binned over pre-defined viability intervals, as shown in figure 7.3(f). 

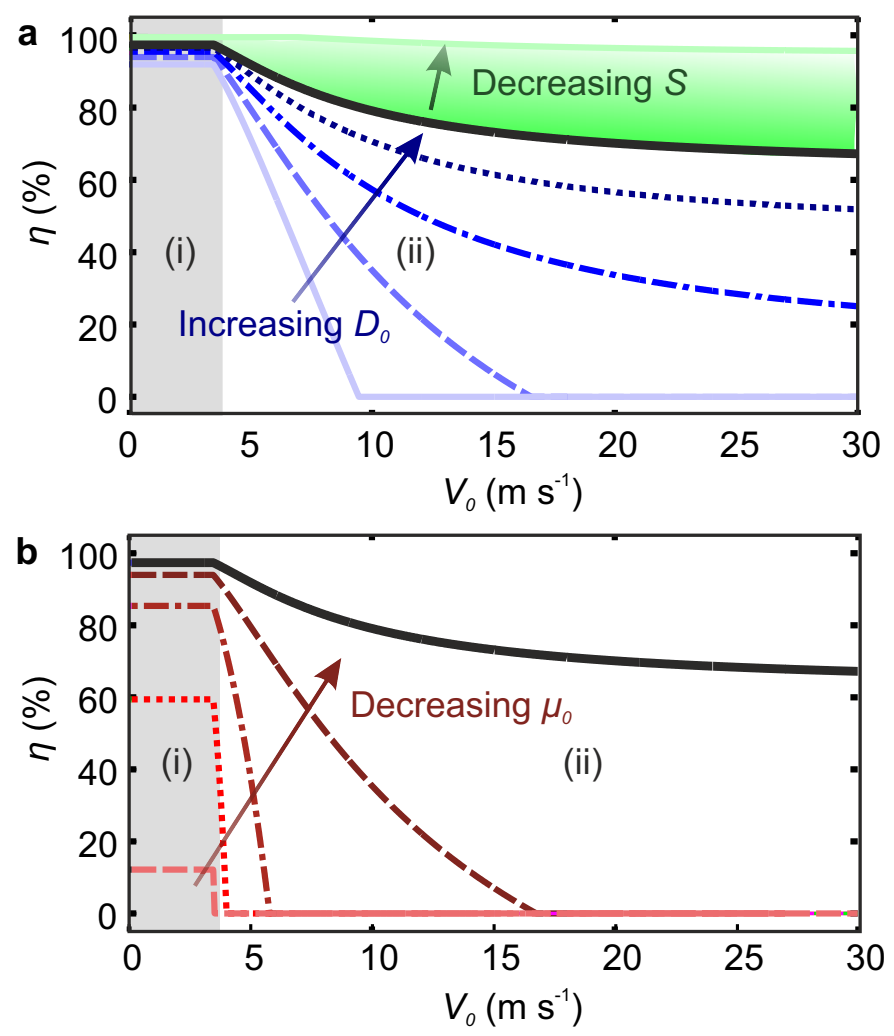

Figure 7.2: Model predictions of the post-impact cell survival probability $\eta$ as a function of the impact velocity $V_{0}$, for a single-cell containing droplet. Figure (a) shows the influence of the droplet diameter $D_{0}$ (indicated by lines representing $1 \leq D_{0} / D_{c} \leq 3$ in steps of 0.5 ) and the surface stiffness (indicated by the color gradient representing $S=1$ (stiff substrate) to $S=0$ (liquid pool)). Figure (b) shows the influence of the droplet viscosity (lines plotter for $\mu_{0}=1,2,4,8,12 \mathrm{mPa} \mathrm{s}$ ). The solid black lines indicate the viability values obtained for the reference parameters: $D_{0} / D_{c}=3, \mu_{c} / \mu_{0}=10$, and $S=1$. Region (i) (shaded) indicates the low-Weber number regime (We $<5$ ). Here the cell deformation is small and independent of the impact velocity. In region (ii), decreasing viability is obtained for increasing velocities, due to increasing cell deformation. 


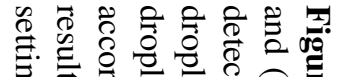

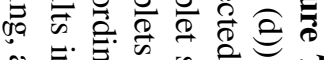

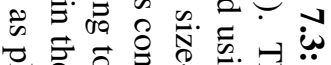

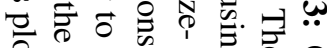

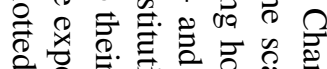

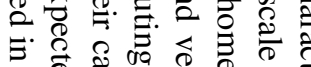

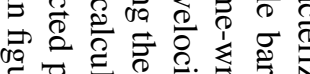

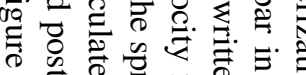

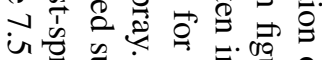

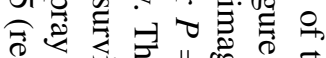

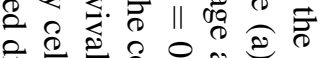

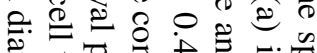
芯

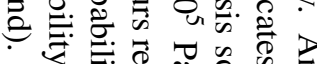

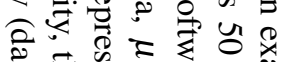

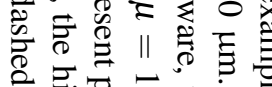
를

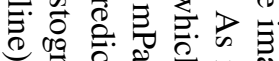

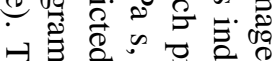

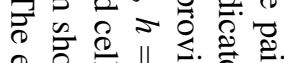
จ $\cong \|$ 定

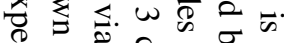
灾

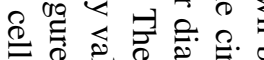

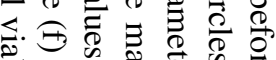

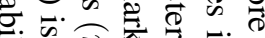

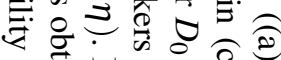

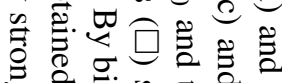

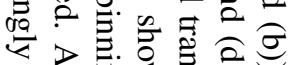

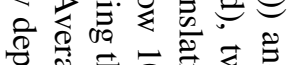
ठํㅠ.

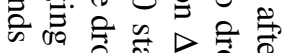

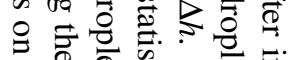

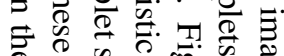

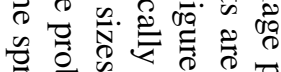
우 $\begin{gathered}0 \\ 0\end{gathered}$

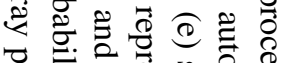
뭉

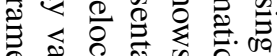

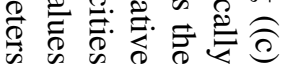
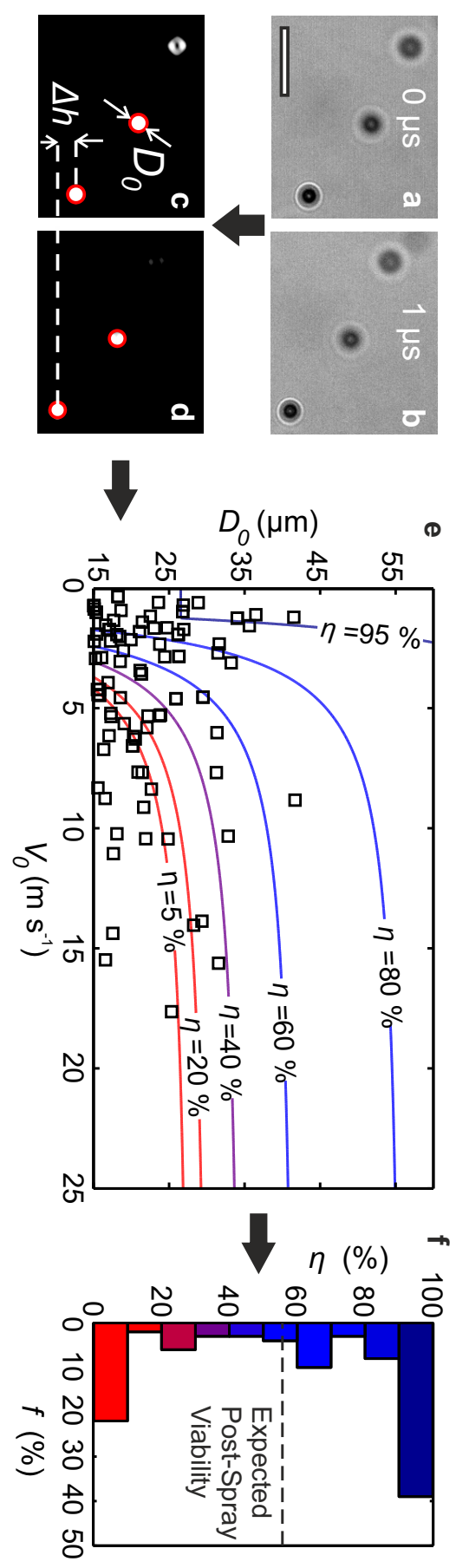
Averaging the viability probabilities then provides the expected viability after a single spraying experiment, which are compared to experimental viability data in the following section.

\subsubsection{Cell viability measurements and model validation}

To measure the post-impact cell viability, spray experiments are performed using the setup displayed in figure 7.1. After spraying, the cells are collected and stained in a live-dead assay of which an example is shown in figure 7.4. Using automated image analysis, the cell viability is determined for each measurement. Figure 7.5 shows the measured cell viability as a function of the pressure, the nozzle-substrate distance, the viscosity, and the surface stiffness.

The model is compared to cell viability measurements in figure 7.5. Good agreement is observed for the pressure, the nozzle-substrate distance, and the surface stiffness. In particular, except for some outliers, the measured data is quantitatively described by the model, which is remarkable in view of the single fitting parameter used. Our model over-estimates the influence of increased viscosity, but still captures the trend. Possibly, the numerical results on which equation 7.7 is based are not valid for our spray, since most of our droplets have a size just exceeding the cell size (figure S3) whereas equation 7.7 is derived from numerical results for much larger cell-containing droplets (ref. [25], also see supplementary section III). Future numerical work assessing the cell survival in small droplets may resolve this issue. Still, the overall agreement between the predicted and the measured cell viability confirms that the mechanism of cell damage is primarily impact-related.

The validated model explains all cell viability trends observed in figure 7.5. For increasing pressure, the cell viability decreases as observed in figure 7.5(a). This trend originates from smaller droplet sizes and faster impact of the spray (see figure 7.6), which both result in stronger cell deformation and therefore cell death. Increasing the nozzle-substrate distance improves the cell viability, as shown in figure 7.5(b). Figure 7.6 shows that this trend is primarily caused by the decreasing droplet velocity far from the nozzle, which results in less deformation of the cell and increased viability. Higher viscosities result in a lower cell viability, as shown in figure 7.5(c). For high viscosity, the deformation of the cell-containing droplet primarily occurs within the cell. Consequently, the cell membrane is significantly stretched, and the viability decreases. Finally, decreasing the surface stiffness improves the viability (figure 7.5(d)), since the deforming surface "cushions" the impacting cell-containing droplet. 

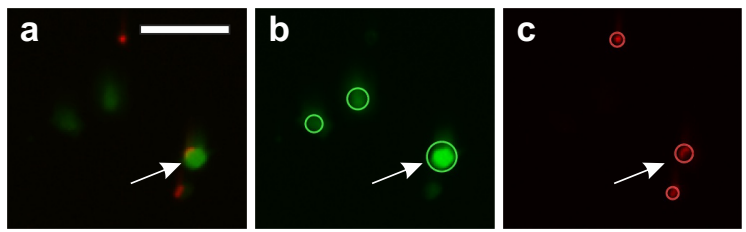

Figure 7.4: Example live-dead assay. Figure (a) shows the original image (the scale bar indicates $100 \mu \mathrm{m}$ ); figure (b) shows the calcein staining (in green), and figure (c) shows the EthD staining (in red). The circles in figures (b) and (c) indicate the automatically detected cells for each staining. The arrows show a cell in which both stainings are retrieved. These cells are considered damaged but viable, and therefore counted as live.

\subsection{Discussion}

Droplet impact-induced cell damage has far-reaching consequences for users as well as developers of cell spraying and other bioprinting technologies.

In clinical practice, the need for adequate cell-spraying protocols [35] is even more pressing than expected. In particular, manual operation of spray devices is common practice, but associated with variations in nozzle-substrate distance, the air pressure, and the viscosity. These variables should be carefully controlled to ensure high cell viabilities. The actual values may still depend on the nozzle design and the cell type, but increasing the spray distance and using low-viscosity spray suspensions, while avoiding hard impact surfaces, will generally improve cell survival.

However, harmful spraying conditions cannot always be avoided due to treatmentspecific clinical requirements or constraints in biofabrication. For example, in arthroscopic procedures, the nozzle-surface distance is limited to at most $1 \mathrm{~cm}$ [4]. In view of our results, it is unclear whether the treatment success shown for burn treatments, where this distance usually exceeds $10 \mathrm{~cm}$, can be reproduced in arthroscopic application (see figure 7.5(b)). Also, in many treatments, the impact surface is a tissue defect and therefore cannot be freely chosen or altered. Our hardest gelatin-containing surfaces result in similarly low viability as hard glass surfaces. These $20 \%$ gelatin surfaces are similar in stiffness to muscle tissue [33], which is one of the softer human tissues [34]. Thus, clinically relevant surfaces are relatively stiff, possibly affecting cell survival. To solve this problem, the spray parameters require optimization. Similarly, the deposition of viscous, cell-containing hydrogels is usually required to preserve the desired 3D tissue architecture in biofabrication [12]. However, such liquids are likely to negatively affect the cell viability (see figure 7.5(c)). Decreasing the spray pressure [2,15-17], using softer impact surfaces [24], or increasing the nozzlesubstrate distance can counteract the negative influence of the increased viscosity (see 
7.3. DISCUSSION

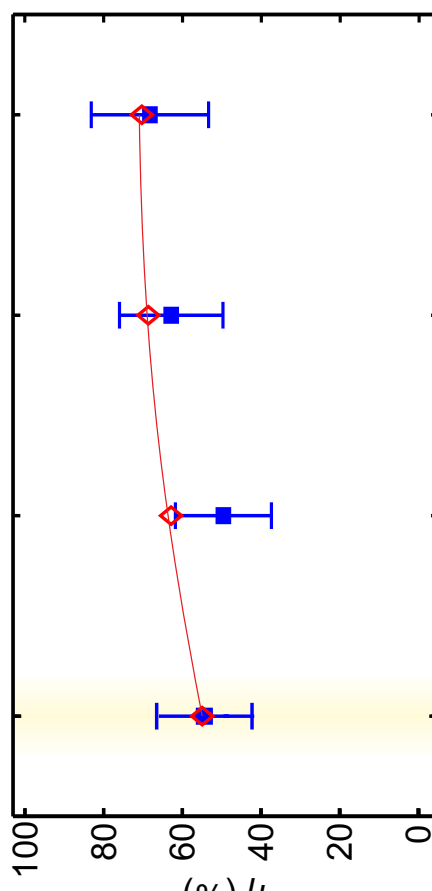

○
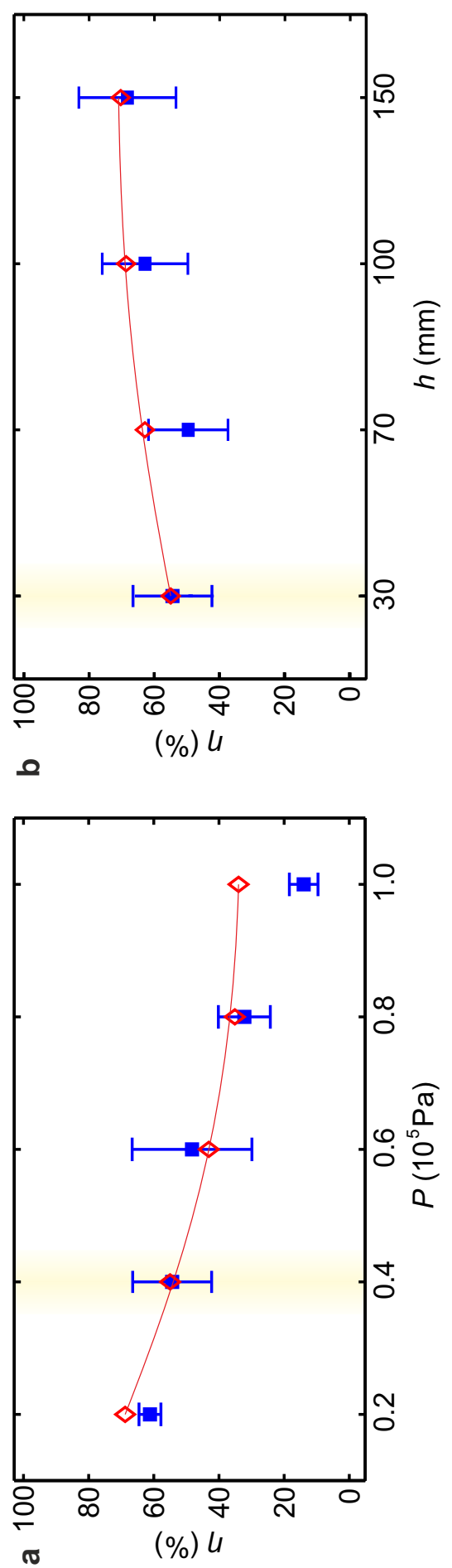

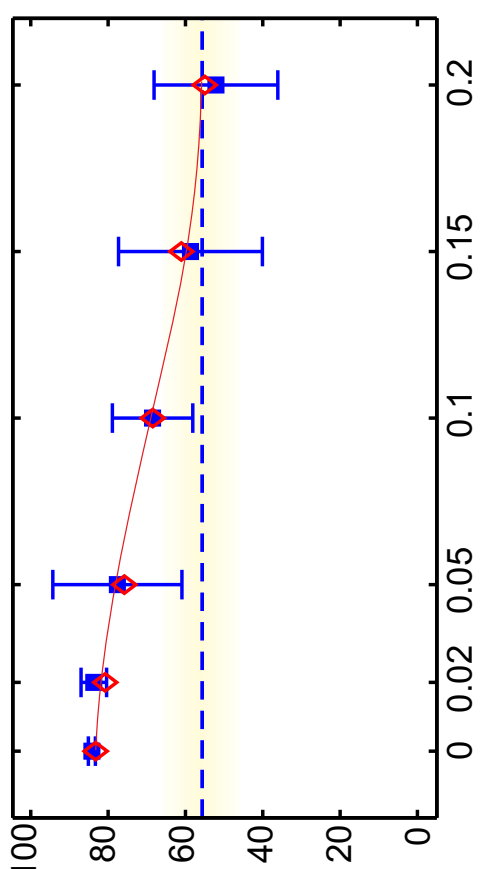

(\%) $u$

б

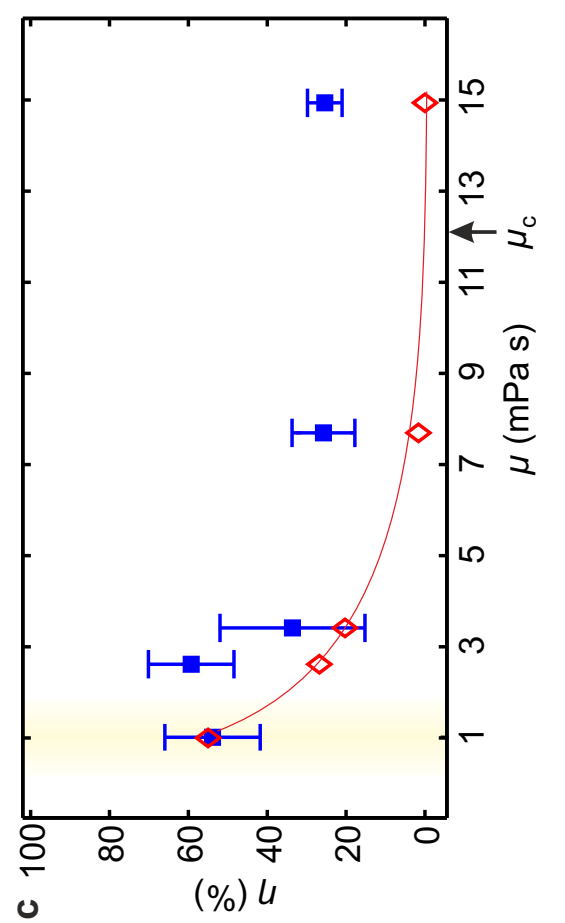

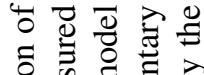

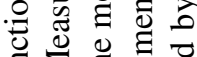
艺要

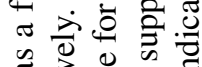
政.

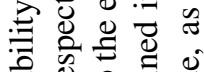
.

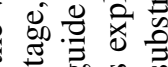
Q

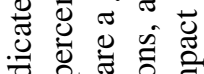
引

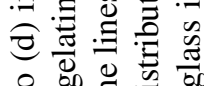
$\circ$ 品里: తิ)

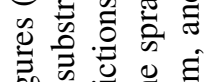
施 江导总苔

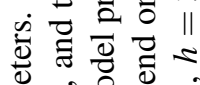
हैं

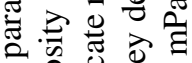
응 : 竞.

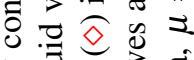

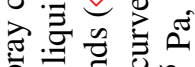
क्ष 0 을 进

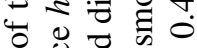

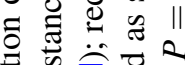

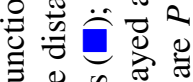

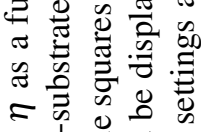

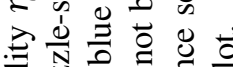
글 $>$ \& $\overline{0} \equiv$

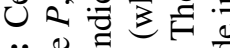

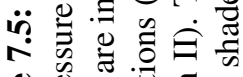

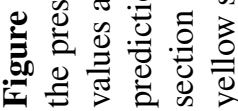




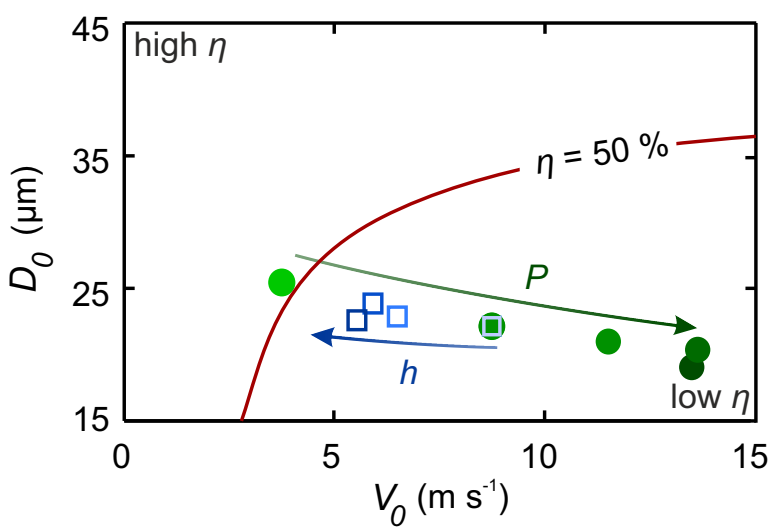

Figure 7.6: Viability as a function of the droplet size and speed. The line represents the $50 \%$ viability contour; additional contours are plotted in figure 7.3 (e). The dots indicate the mean diameter versus the mean velocity as a function of the spray pressure (the arrow indicates increasing pressure for $P=[0.2,0.4,0.6,0.8,1] \cdot 10^{5} \mathrm{~Pa}$ ). The open squares indicate the distance from the nozzle for $h=[30,50,100,150] \mathrm{mm}$. For increasing pressure, the droplet size decreases and the impact velocity increases, resulting in a lower viability. For a larger distance from the nozzle, the impact speed decreases, resulting in improved viability. The influence of the viscosity and the surface stiffness is displayed in figure 7.2, since these variables do not affect the droplet size and speed.

figure 7.5), but, unfortunately, these parameters are sometimes also constrained.

The greatest potential to improve cell viability in cell spraying therefore seems to be optimization of spray nozzle designs. Particularly, design optimization resulting in increased and monodisperse droplet sizes and reduced impact velocities would allow for successful cell deposition in an extended viscosity range. In this study we have used the Duploject system, which is approved for clinical application of fibrin glue by spraying and also used for cell spraying [4]. The spray produced by this nozzle is characterized by highly polydisperse droplet diameters and velocities. High-velocity impacts occur even for the most gentle spraying parameters, limiting the measured highest post-spray cell viabilities to $90 \%$. Nozzle designs producing more monodisperse droplets may prevent these lethal events, even for high-throughput spraying of viscous liquids. Our experimental setup can be used to rationally optimize such future spray nozzles, which may substantially enhance the application window of cell-spraying.

Finally, other droplet-based cell deposition technologies may benefit from our approach. In ink-jet bioprinting, highly monodisperse cell-containing droplets [36-39] are deposited. Here, typically, low-viscosity droplets of $40 \mu \mathrm{m}$ (exceeding the cell 
size by a factor of 3) impact at velocities below $10 \mathrm{~ms}^{-1}$. Good viability is generally measured in this range [23], in agreement with our model (figure 7.2). However, reduced cell viability is observed for neural cells [40]. Our study suggests that especially decreasing the liquid viscosity or reducing the droplet ejection velocity could improve the cell viability of such more fragile cell types. These measures reduce the expected cell damage both in the nozzle and during impact, which are both likely causes of cell damage in ink-jet printing (see supplementary section 1). Using soft impact substrates or larger nozzle-substrate distances will still reduce impact-related damage, but nozzle-induced cell damage or poration [10] cannot be suppressed in this manner.

Cell viability trends observed in laser-assisted bioprinting (LAB) are also described by our model. Here, a pulsed laser is focused onto a cell-containing liquid film, resulting in the deformation of this film and break-up into cell-containing droplets [41, 42]. By placing a receiver substrate in the line-of-flight of the cellcontaining droplet, deposition is achieved. Increased impact velocities result in decreased cell viability [43, 44], and soft impact surfaces improve cell survival [45], analogous to our model results shown in figure 7.2. Surprisingly, improved cell viability was reported for increased viscosity [44], but this is likely explained by the reduced impact velocity due to the increased viscosity. Therefore, studying the cell viability as a function of the liquid viscosity at a controlled impact velocity would be highly interesting. Such experiments may also advance the understanding of cell membrane deformation due to pulsed shear stresses [46], as occurring in cellcontaining droplet impact.

In conclusion, we present and validate an analytical model describing the cell viability as a function of the droplet impact parameters. The model accurately describes cell-viability trends in both cell spraying and laser-assisted cell transfer, confirming the general importance of droplet impact for cell survival in bioprinting. Since future biofabrication applications may involve high-throughput deposition of different, possibly more fragile, cell types contained by high-viscosity bio-inks, we expect that prevention of cell damage will become even more important. In particular, postspray cell survival will be cell-type dependent. In addition, different bio-inks may require distinct deposition parameters in combination with dedicated nozzle designs allowing monodisperse droplet ejection while maintaining low shear rates inside the nozzle. Our study provides a framework to optimize cell survival in such future applications, contributing to reliable biofabrication of complex 3D-tissue constructs of a clinically relevant size. 


\subsection{Methods}

Cell culture. Neonatal rat dermal fibroblasts (ITK Diagnostics) were cultured in Minimum Essential Medium $\alpha$ ( $\alpha$-MEM) (Gibco) supplemented with $10 \%$ Fetal Bovine Serum (FBS) (Lonza), 1\% L-Glutamine (Gibco) and 1\% Pen/Strep (Gibco) at $37^{\circ} \mathrm{C}$ and $5 \% \mathrm{CO}_{2}$.

Cell suspension. Cells were harvested at $80 \%$ confluence by trypsinization and suspended at $1.5 \times 10^{6}$ cells per $\mathrm{ml}$ in culture medium excluding FBS for impact experiments. Optionally dextran (Sigma, 15-25 kDa) was added to the cell suspensions to increase the viscosity. The influence of the dextran concentration on the liquid viscosity was measured using a viscometer (Rheolab QC, Anton Paar). As shown in figure S10, the measured viscosity is in agreement with literature values.

Cell viability measurements. Cell spray experiments were performed with the set-up shown in figure 7.1. Using a syringe pump, the cell suspension was pushed through a spray nozzle (Duploject spray system (Baxter AG). Photographs of this system, which is also known as bio-airbrush, are provided in figure S9) at a controlled liquid flow rate of $2.4 \mathrm{ml}$ per min. The impact surface consisted of a standard clean microscope slide covered with a PDMS mask to ensure a defined and reproducible impact area. These slides were optionally coated with a layer of gelatin (Type A, Sigma, $d=0.5 \mathrm{~mm}$ ), in order to adjust the surface stiffness. A crucial aspect of the experiment is that impact occurs on a dry surface, i.e. that the cell spray does not impact onto previously sprayed droplets. To ensure this, the impact surface was reproducibly moved using a programmable linear motor. The substrate velocity was set such that $12 \pm 0.5 \mathrm{mg}$ of the sprayed liquid was collected for each experiment (the velocity was decreased for increasing nozzle-substrate distances since the spray density decreases for increasing distance from the nozzle). This weight corresponds to a liquid film of $18 \mu \mathrm{m}$ (which is equivalent to $\sim 1$ cell thickness) and resulted in covering roughly half of the surface area with sprayed droplets, such that most droplets land on the dry surface. Within $10 \mathrm{~s}$ after each spray experiment, the surface was rinsed with FBS-free culture medium to collect the cells. The cells rinsed from three different samples (sprayed using equal parameter settings) were collected into a $12 \mathrm{ml}$ centrifuge tube (Greiner). The contents of each tube were subsequently processed in a live-dead assay. For each parameter setting, three independent spray cycles were performed (i.e. 9 impact surfaces were collected in three different tubes). This approach provided three data points for each parameter setting, which were used to display the error bar in figure 7.5. 
Live-dead assay. The collected cells were incubated in phosphate buffered saline (PBS), supplemented with $1 \mathrm{nM}$ calcein $\mathrm{AM}$ and $6 \mathrm{nM}$ ethidium homodimer, at $37^{\circ} \mathrm{C}$ and $5 \% \mathrm{CO}_{2}$ for 30 minutes. Each stained cell suspension sample was transferred to a well in a 24 wells-plate and 8 random spots per well were imaged (EVOS Fl microscope). Live (green) and dead (red) cells were automatically counted using a home-written Matlab script (figure 4 shows an example), resulting in an average of $450 \pm 332$ (s.d.) detected cells per sample (with a minimum of 12 cells). Cells stained both live and dead were considered damaged, but viable, so counted as alive. For each sample, viability was calculated accordingly. All measurements were subsequently corrected by setting the control viability (non-impact, measured at the same time) to $100 \%$.

Spray characterization. As cell survival critically depends on the characteristics of the cell-containing droplets, the spray characteristics were determined in detail. The setup used is shown in figure S1. All spray-generating components were equal to the components used for assessment of the cell viability (figure 7.1). A dual-pulse ND:Yag laser with a pulse duration of $6 \mathrm{~ns}$ was used for brightfield illumination. To prevent fringes, the coherent laser light was diffused using a fluorescent plate placed in front of the laser. The non-coherent pulses were captured by a dual-shutter camera (Sensicam, PCO). The time delay between the illumination pulses was set to $1 \mu \mathrm{s}$, which is sufficiently long to measure the translation $\Delta h$ of the droplets while preventing confusion between different droplets. All timings were controlled using a BNC 575 pulse-delay generator (not shown). A $10 \times$ long-distance objective was used, resulting in a field of view of $0.67 \times 0.89 \mathrm{~mm}^{2}$. As the focal plane thickness of this objective is $\delta_{F} \approx 0.1 \pm 0.03 \mathrm{~mm}$, a volume of $0.67 \times 0.89 \times 0.1 \mathrm{~mm}^{3}$ was visualized. As the spray is much larger than this volume, measurements were taken at different $z$ - and $h$-positions to fully characterize the spray, as illustrated by the small rectangles in figure $\mathrm{S} 1$ (b) (the $r$-position is maintained at the nozzle axis).

For each measurement, 400 image pairs were obtained. Example images are shown in figures 7.3(a) and (b), where the downward translation of the droplets is clearly visible. Motion blur was prevented by the short illumination pulses of $6 \mathrm{~ns}$. The droplets were automatically detected using a home-written Matlab script. Only droplets appearing sufficiently sharp were processed, as illustrated by the red circles in figure 7.3(c) and (d). Here, the top-left droplet was too blurred for detection and discarded. Although the droplets in figure 7.3 are similar in size, the image processing software allowed for successful detection of droplets in the range of $1 \leq D_{0} \leq$ $100 \mu \mathrm{m}$.

Soft substrate preparation. Glass microscope slides were optionally coated 
with a layer of gelatin (Type A, Sigma) of thickness $0.5 \mathrm{~mm}$, and kept in air at room temperature for $30 \mathrm{~min}$ prior to the experiment. The surface stiffness was adjusted by adding gelatin mass fractions of $C_{g}=[2,5,10,20] \%$ to PBS. PBS without gelatin was used in the soft-surface limit.

\subsection{Supplementary material}

I. Shear rate analysis The shear rates in the spray nozzle and during impact are estimated. The shear rate is defined as $\gamma(y)=\partial u / \partial y$, with $u(r)$ the velocity in $r$ direction. The coordinate system shown in figure $\mathrm{S} 1$. By dimensional analysis the shear rate during impact is estimated as $\gamma \sim V_{0} / D_{0} \approx 10^{6} \mathrm{~s}^{-1}$ for a $D_{0}=10 \mu \mathrm{m}$ droplet impacting at $10 \mathrm{~ms}^{-1}$. For drop-on-demand systems (i.e. ink-jet printing, valve-based deposition, and certain regimes of laser-induced forward transfer) both the velocity inside the nozzle and the diameter of the nozzle are of the same order as $D_{0}$ and $V_{0}$, respectively. Therefore, distinguishing the nozzle-induced shear from the impact-induced shear is far from trivial. However, for the cell spray, the diameter of the nozzle $D_{N} \sim 500 \mu \mathrm{m}$ is much larger than $D_{0}$, resulting in a strongly reduced shear rate within the nozzle, which is estimated as $\gamma \sim V_{0} / D_{N} \approx 2 \cdot 10^{4} \mathrm{~s}^{-1}$. Therefore, only the impact of cell-containing droplets is expected to have a significant shear-induced effect in cell spraying.

\section{Spray characterization}

As described in the Methods section, the spray is characterized using the setup shown in figure S1. Supplementary figure S2 shows images of the spray as a function of the air pressure similar to observation with the naked eye. The spray visually changes as a function of the pressure and widens with increasing distance from the nozzle. Some droplets are individually visible, but these only represent the and largest and slowest droplets.

Key spray characteristics are shown in figure S3. Supplementary figures S3 (a) and (b) respectively show that the droplet diameter decreases for increasing pressure, and that the droplet velocity increases for increasing pressures. Supplementary figure S3 (c) illustrates the strong influence of the nozzle-substrate distance on the spray velocity. In addition, we found that the nozzle-substrate distance hardly affects the droplet diameter, and that the viscosity neither affects the droplet diameter nor the droplet velocity (not shown).

The influence of the spray characteristics on the cell viability is shown in figures S4 to S7. Here, the droplet size- and velocity distributions are plotted for a range of spraying parameters (left figures). In each plot, the corresponding viability probabil- 

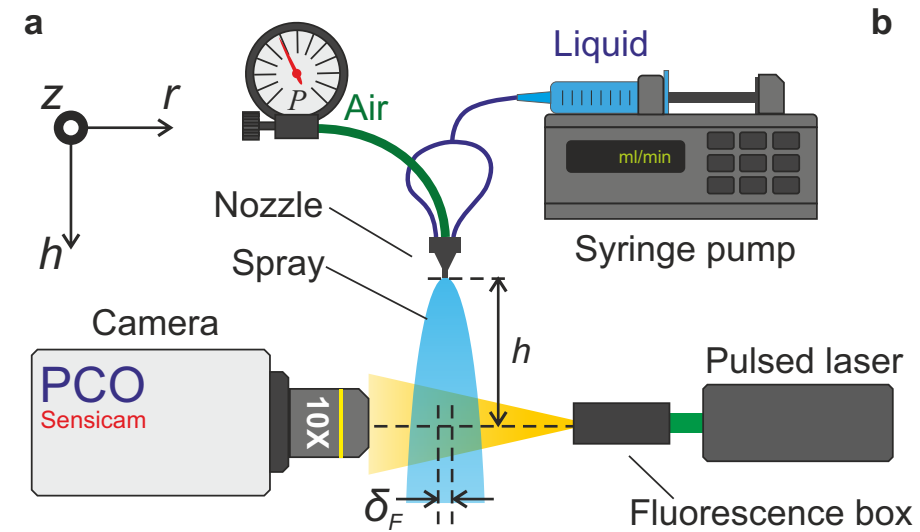

b

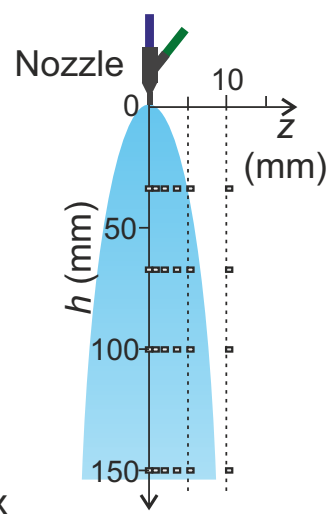

Figure S1: (a) Overview of the setup used for the spray characterization measurements. For visualization, a dual-shutter camera is used. For each image a slice of the spray is visualized, corresponding to the depth of the focal plane $\delta_{F}$. Illumination is provided by dual-pulse ND:Yag laser combined with a fluorescence box to remove the laser coherence. The spray generation is equal to the cell spraying experiments. (b) Side-view of the spray. Each small rectangle indicates a measurement location used for spray characterization, corresponding to the field of view of the camera (drawn to scale).

ity contours are also plotted. For increasing pressure, the droplet size decreases and the droplet velocity strongly increases, which results in more low-viability impacts as observed by comparing figure S4 (a) and (c). This trend is reflected in figure S4 (d), where almost half of the cells will certainly not survive the impact. Increasing the distance from the nozzle primarily decreases the impact veloctity, as shown in figure S5 (a) and (c). Consequently, the viability increases as shown in figure S5 (d).

Increasing the viscosity hardly affects the droplet size- and velocity-distributions. However, for a given droplet size and velocity, the viability dramatically decreases, resulting in a strong shift of the viability contours shown in figure S6. Similarly, the viability contours change as a function of the substrate stiffness, resulting in increased viability for softer surfaces as shown in figure S7. 

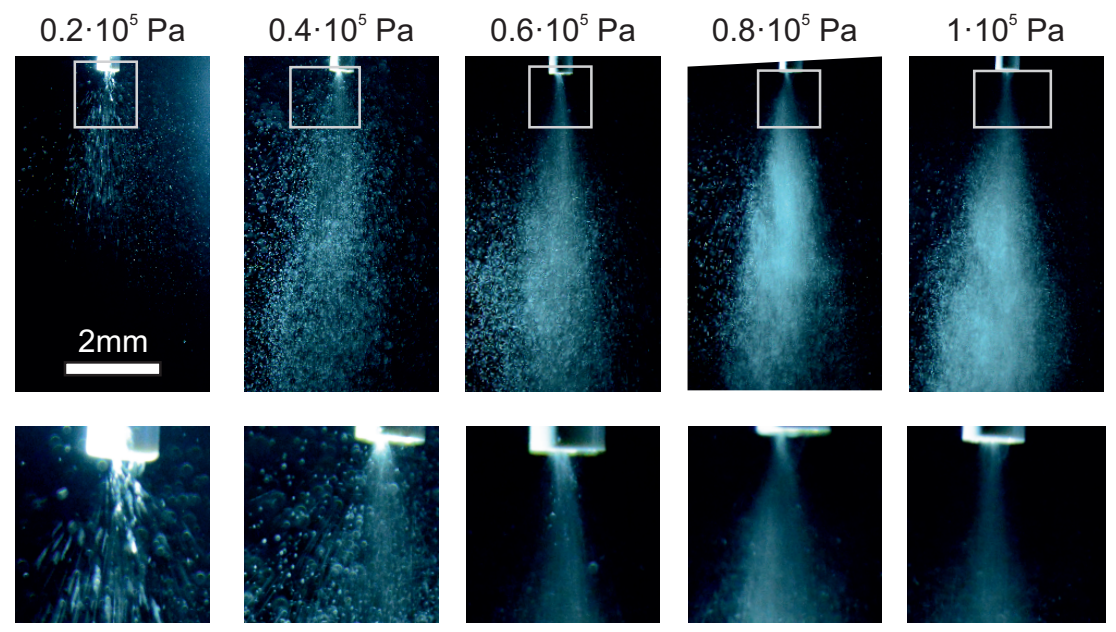

Figure S2: Low-speed images of the spray as a function of the air pressure. Each bottom images represents a magnification of the white rectangle in the top image. For $P=0.2$. $10^{5} \mathrm{~Pa}$ the droplets in the spray appear as stripes, due to motion blur. At higher pressures the individual droplets within the spray are no longer visible. The droplets which appear spherical fall through the surrounding air at very low velocity (i.e. they are not part of the main spray), and constitute a nebula around the spray.
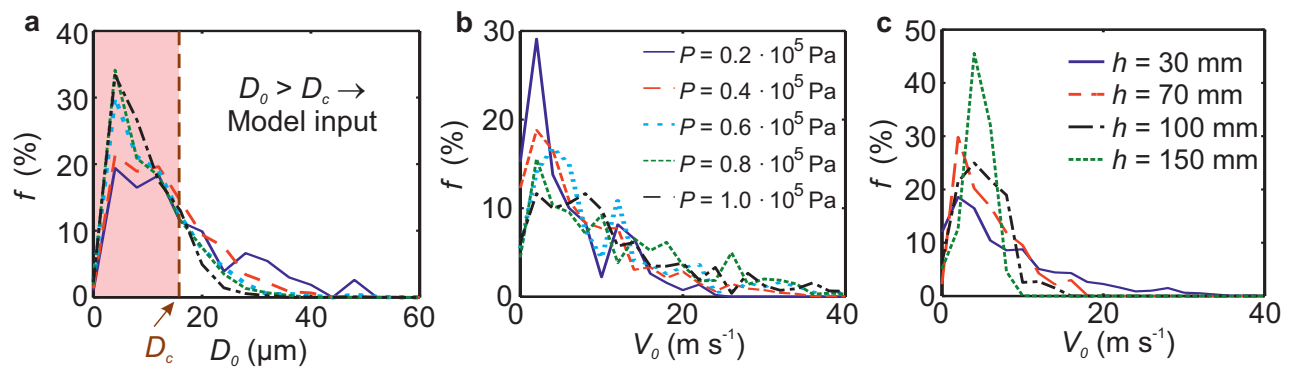

Figure S3: Figure (a) shows the spatially-averaged droplet diameter distribution for different air pressures (legend in figure (b)). The shaded area indicates droplet sizes $D_{0}$ smaller than the cell size $D_{c}=15 \mu \mathrm{m}$, which are omitted in cell viability predictions. Figures (b) and (c) show the droplet velocity distributions for different air pressures (b) and distances from the nozzle (c), for droplets diameters exceeding the cell size. Unless specified in the legend, $P=0.4 \cdot 10^{5} \mathrm{~Pa}, \mu=1 \mathrm{mPa} \mathrm{s}, h=30 \mathrm{~mm}$, and $z=0 \mathrm{~mm}$. 

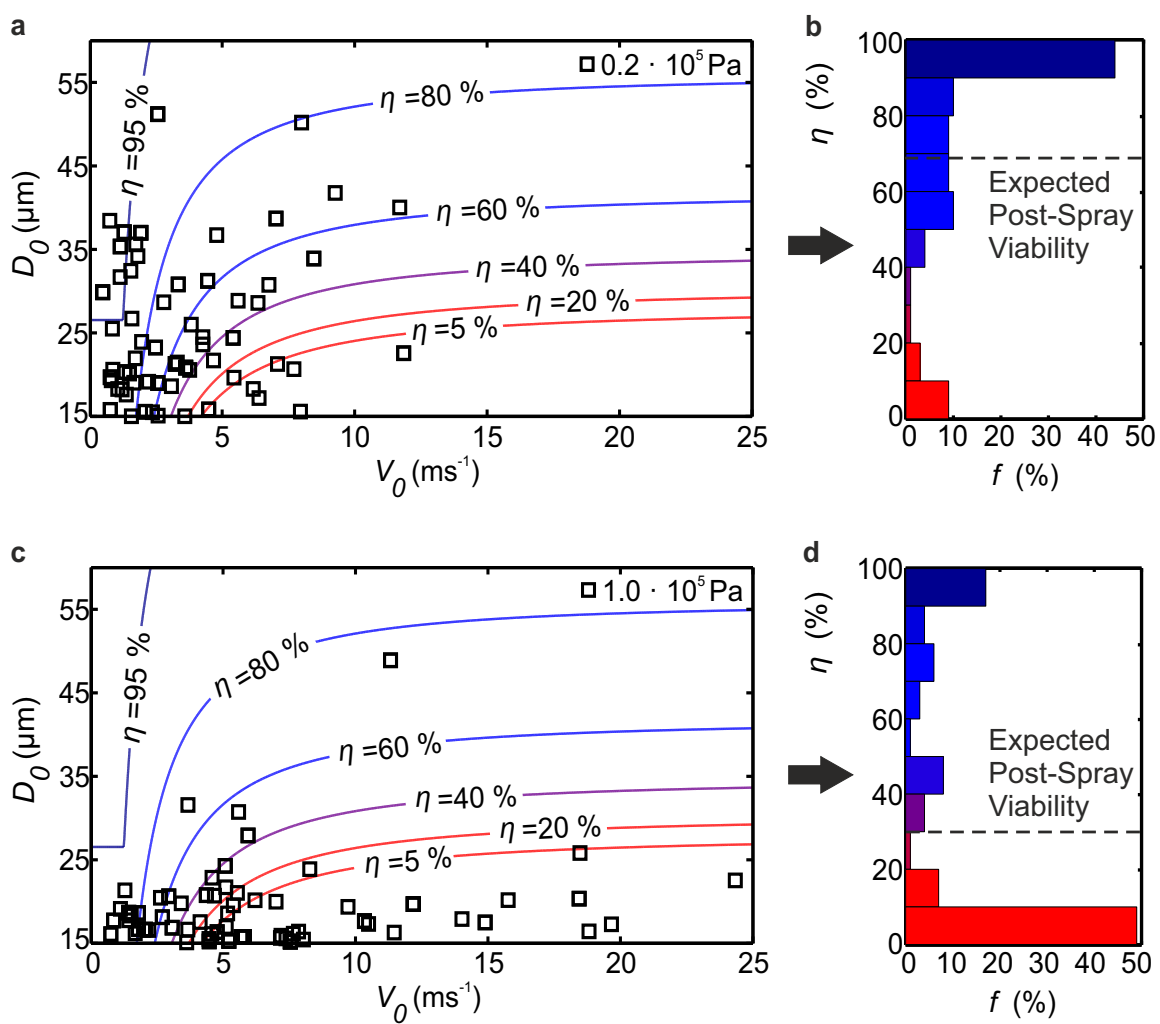

Figure S4: Droplet size- and velocity for a pressure $P=0.2 \cdot 10^{5} \mathrm{~Pa}$ (a) and $P=1 \cdot 10^{5} \mathrm{~Pa}$ (c). Representative cell viability contours are plotted in both figures. For $P=1 \cdot 10^{5} \mathrm{~Pa}$, more small and fast droplets are observed, which corresponds to low-viability regions of the diagram, and a lower predicted cell viability. Cell viability distributions are shown in figures (b) and (d), for pressure $P=0.2 \cdot 10^{5} \mathrm{~Pa}(\mathrm{c})$ and $P=1 \cdot 10^{5} \mathrm{~Pa}(\mathrm{~d})$. The graphs are obtained analogous to figure 3(f) in the main text. The other control parameters are maintained constant at nozzle-substrate distance $h=30 \mathrm{~mm}$, viscosity $\mu=1 \mathrm{mPa}$, and a glass impact surface for which stiffness $S=1$. 

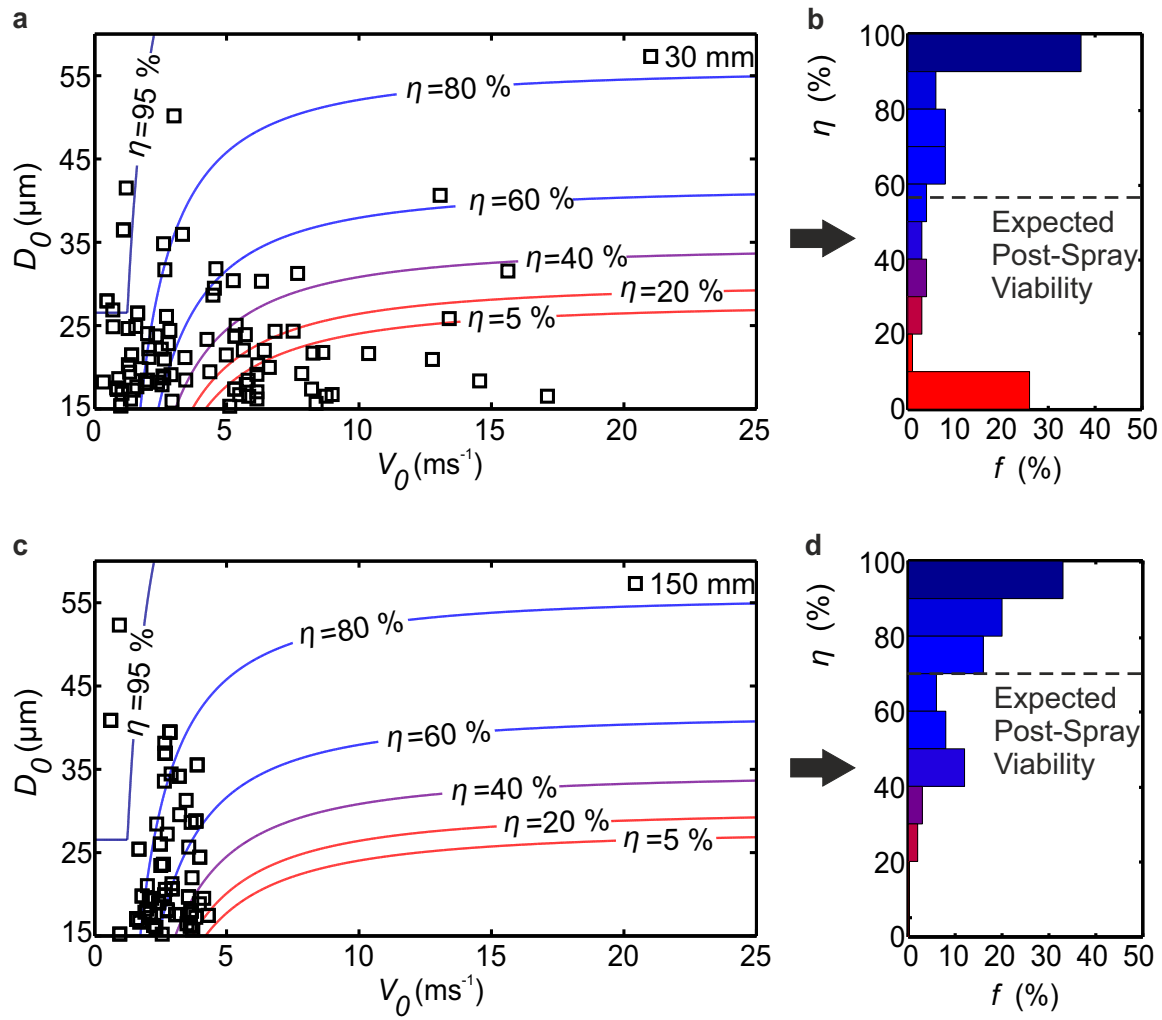

Figure S5: Droplet size- and velocity for a nozzle-substrate distance $h=30 \mathrm{~mm}$ (a) and $h=150 \mathrm{~mm}$ (c). For increasing distance, primarily the droplet velocity is decreased and the distribution corresponds to a high-viability region of the diagram. The increased viability is reflected in the cell viability distributions as shown in figures (b) and (d), respectively. The other control parameters are maintained constant at $P=0.4 \cdot 10^{5} \mathrm{~Pa}$, viscosity $\mu=1 \mathrm{mPa}$, and a glass impact surface for which stiffness $S=1$. 

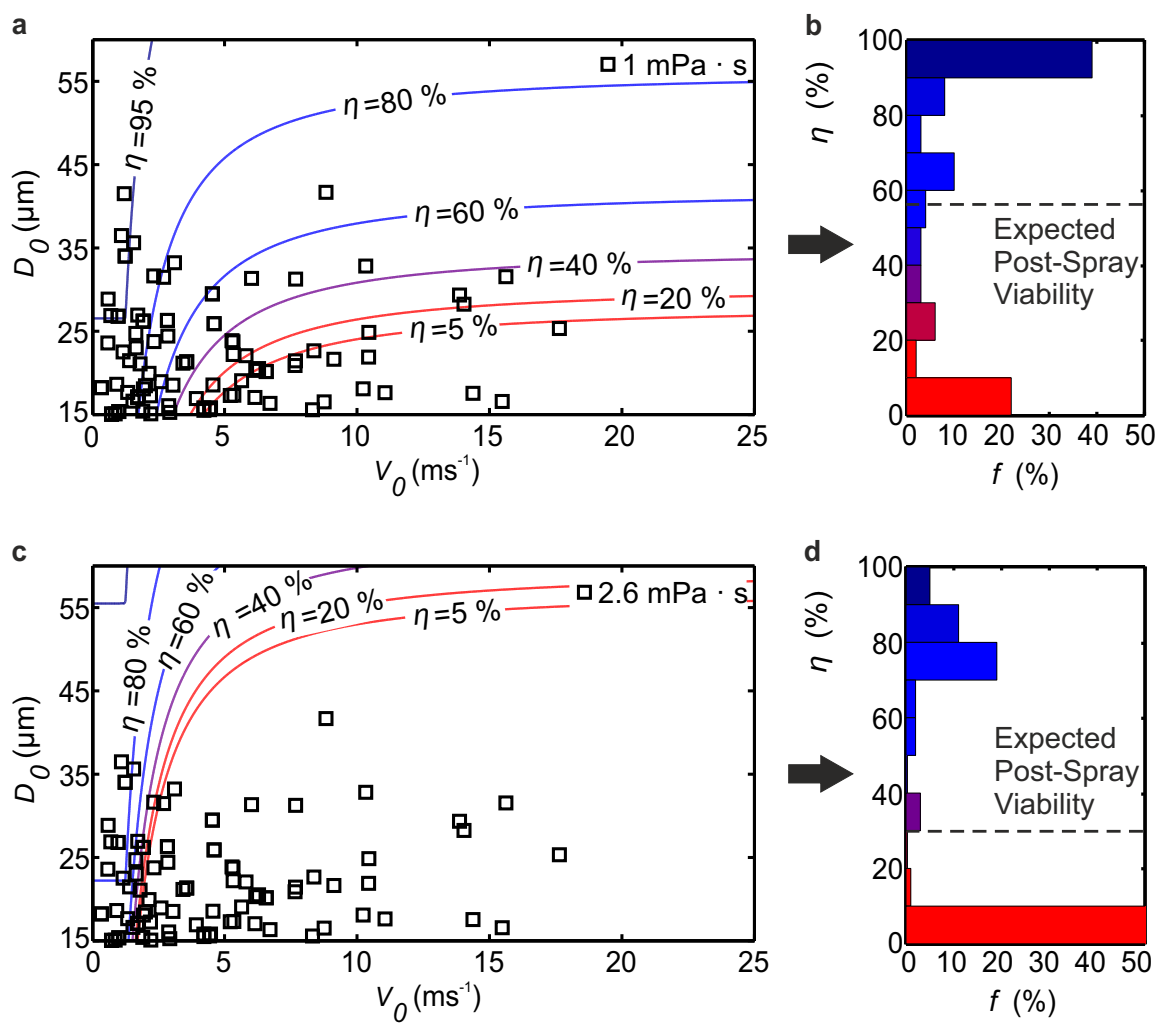

Figure S6: Droplet size- and velocity for a liquid viscosity $\mu=1 \mathrm{mPa} \mathrm{s}$ (a) and $\mu=2.6 \mathrm{mPa}$ $\mathrm{s}(\mathrm{c})$. The increasing viscosity hardly affects the droplet size- and velocity distribution. However, the expected viability sharply drops for a constant droplet size and speed, which is reflected by a change in the viability contours in figure (c) as compared to (a). The reduced viability is reflected in the cell viability distributions as shown in figures (b) and (d), respectively. The other control parameters are maintained constant at pressure $P=0.4 \cdot 10^{5} \mathrm{~Pa}$, nozzle-substrate distance $h=30 \mathrm{~mm}$, and a glass impact surface for which stiffness $S=1$. 

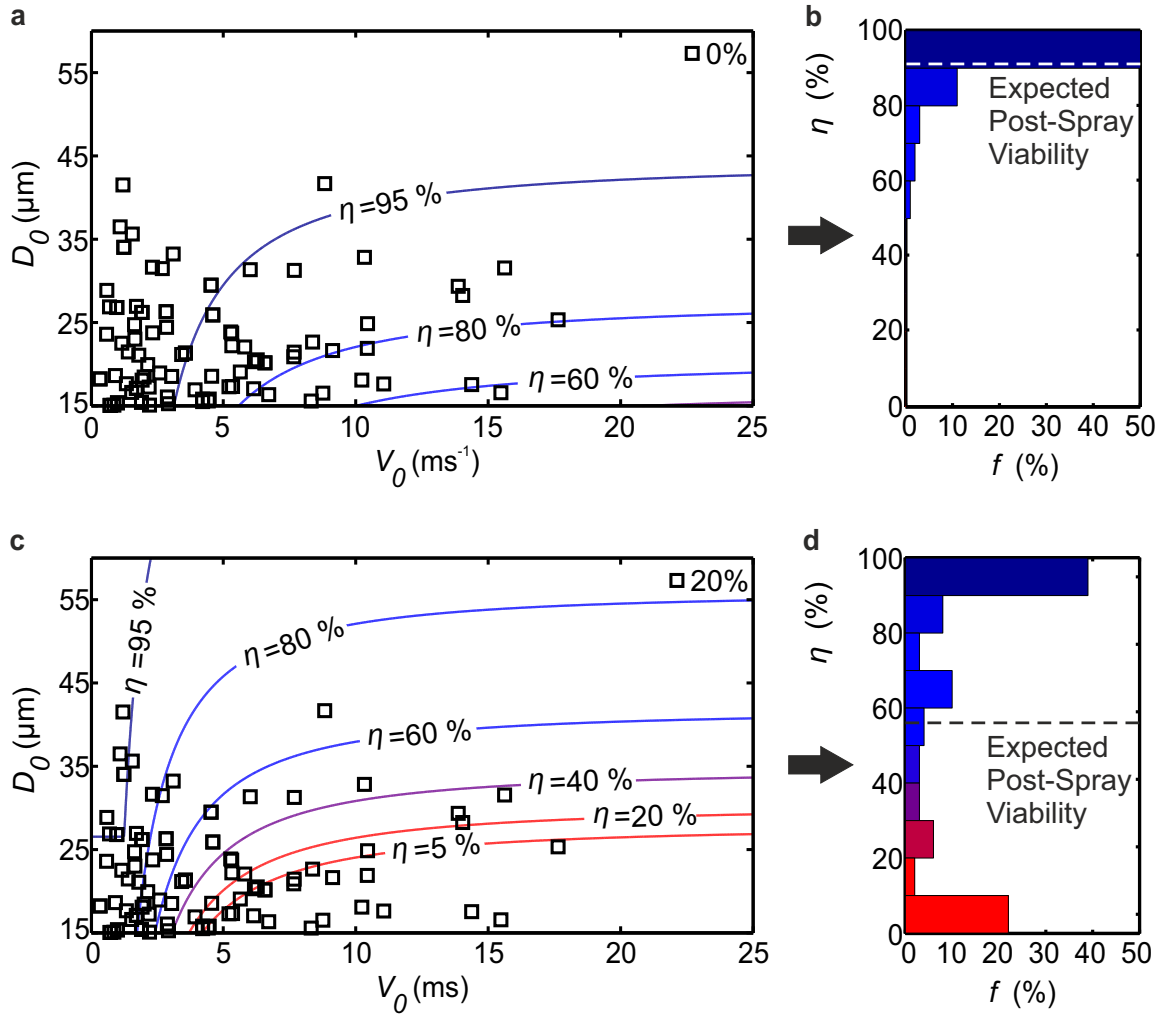

Figure S7: Influence of the substrate gelatin percentace: $C_{g}=0 \%$ in figure (a) (water surface, corresponding to stiffness $S=0$ ) and $C_{g}=20 \%$ in figure (c) (corresponding to stiffness $S=1$ ). The expected viability increases for a constant droplet size and speed, which is reflected by the difference between the viability contours in figure (c) as compared to (a). The increased viability is reflected in the cell viability distributions as shown in figures (b) and (d), respectively. The other control parameters are maintained constant at pressure $P=0.4 \cdot 10^{5} \mathrm{~Pa}$, nozzle-substrate distance $h=30 \mathrm{~mm}$, and viscosity $\mu=1 \mathrm{mPa}$. 


\section{Influence of the surrounding droplet on cell deformation}

The influence of the cell diameter and impact velocity on cell deformation (equations (4) and (5) of the main text) are obtained from basic models describing droplet impact [29]. The influence of the surrounding droplet's diameter $D_{0}$ and viscosity $\mu$ on the cell deformation are obtained from the numerical results in ref. [25], as incorporated in equation (7) of the main text (the procedure is discussed below).

To obtain the influence of the surrounding droplet's diameter $D_{0}$ and viscosity $\mu$ on the cell deformation, the maximal cell deformation values provided in figures 15 and 19 in ref. [25] are extracted and plotted in figure S8 (a) and (b). A priori, the scaling of $M$ as a function of these parameters is unknown. Therefore, different fit functions were tested and the best results are indicated by the dashed lines within these plots (the functional form is provided by the equations). Supplementary figure S8 (c) shows $M$ as a function of both control parameters. Finally, figure S8 (d) shows the predicted increase in surface area as a function of the surrounding droplet's diameter $D_{0}$ and viscosity $\mu$, using $\gamma$ and equation (8) from the main text.

In our work, other relevant control parameters (e.g. surface tension and contact angle [25]) are not varied, and therefore captured by a constant prefactor $C_{0}$ in equation (7) of the main text. Fitting $C_{0}$ to our reference measurement (for which $P=0.4 \cdot 10^{5} \mathrm{~Pa}, \mu=1 \mathrm{mPa} \mathrm{s}, h=30 \mathrm{~mm}$, and a glass impact substrate) provides $C_{0}=5$. 

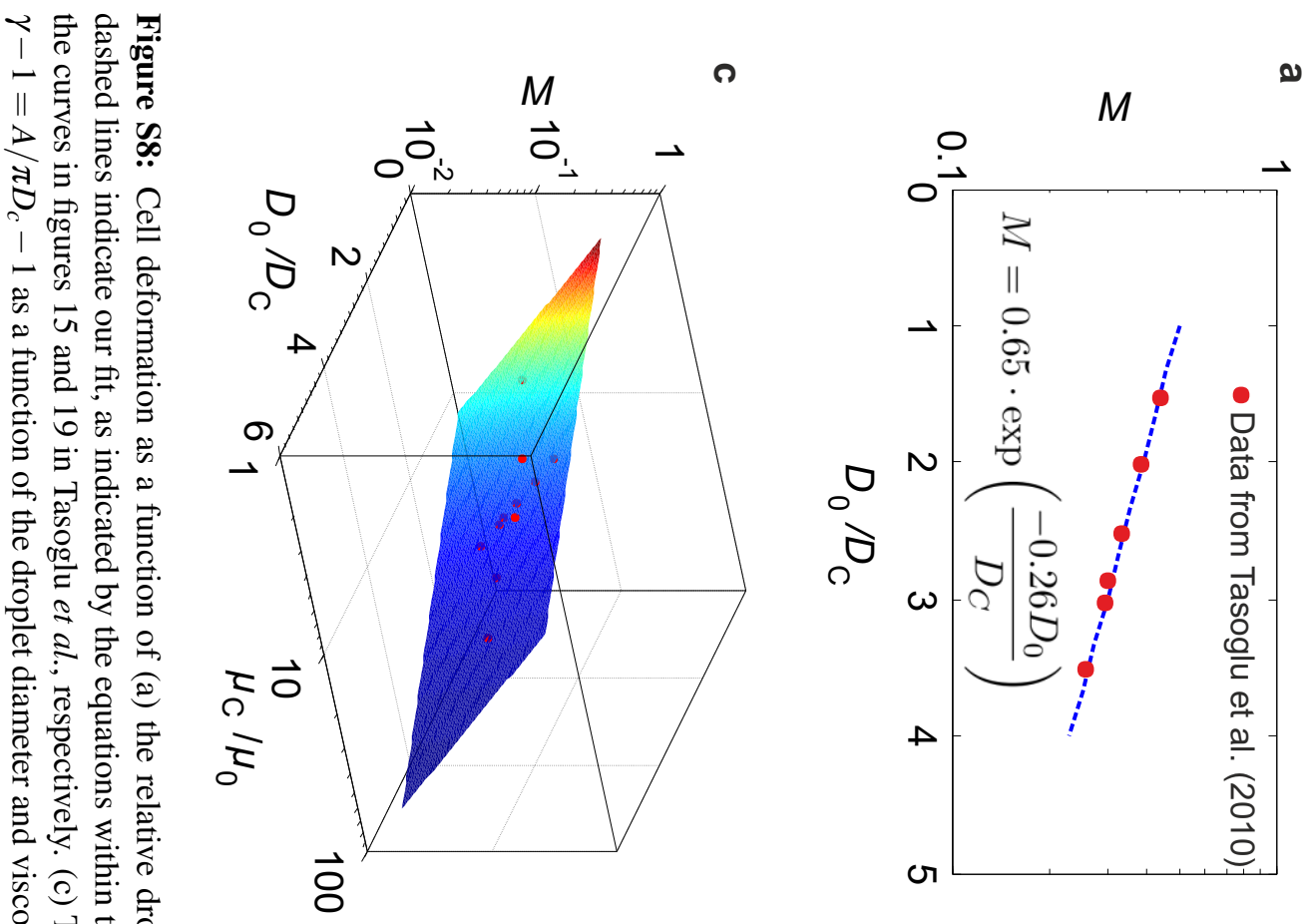

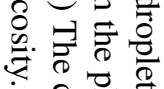
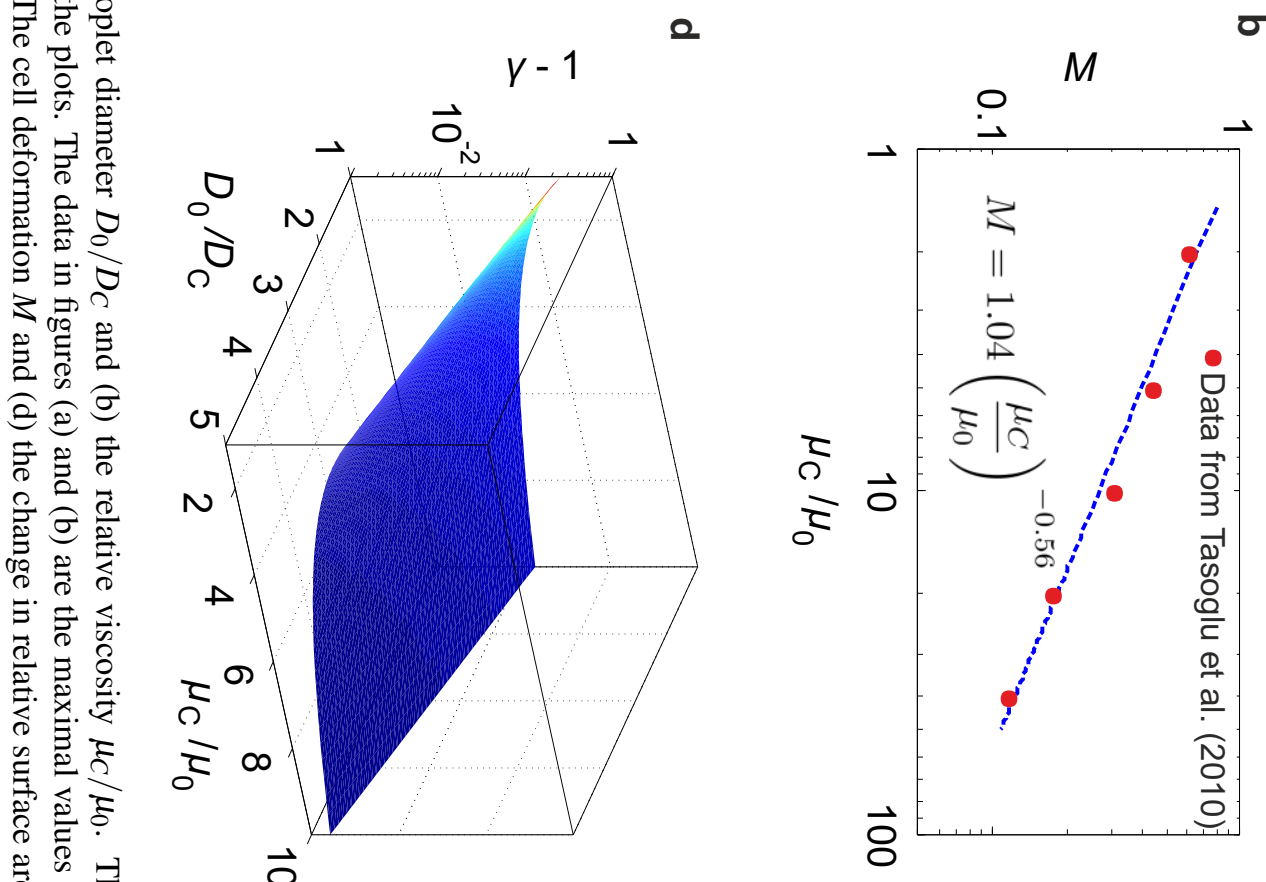


\section{Material characterization}

See figure S9 for images of the spray nozzle, and figure S10 for the measured dextran viscosity.
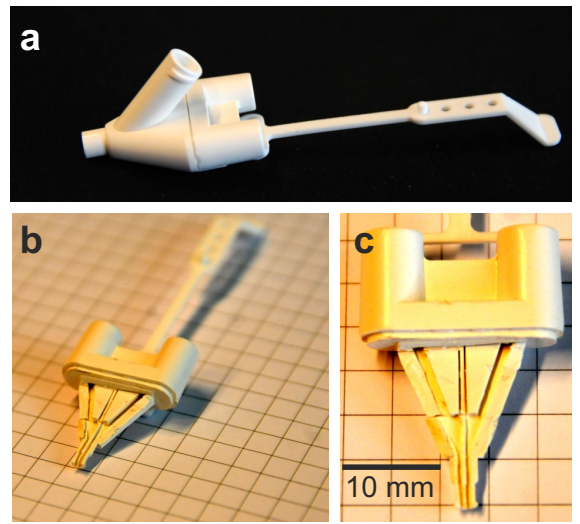

Figure S9: Images of the DuploJect nozzle used. (a) Overview image. The air enters through the top inlet, the liquid enters through the side inlets. (b) Nozzle that is cut to reveal the internal structure (the air inlet is also removed). (c) Top-view of the cut nozzle.

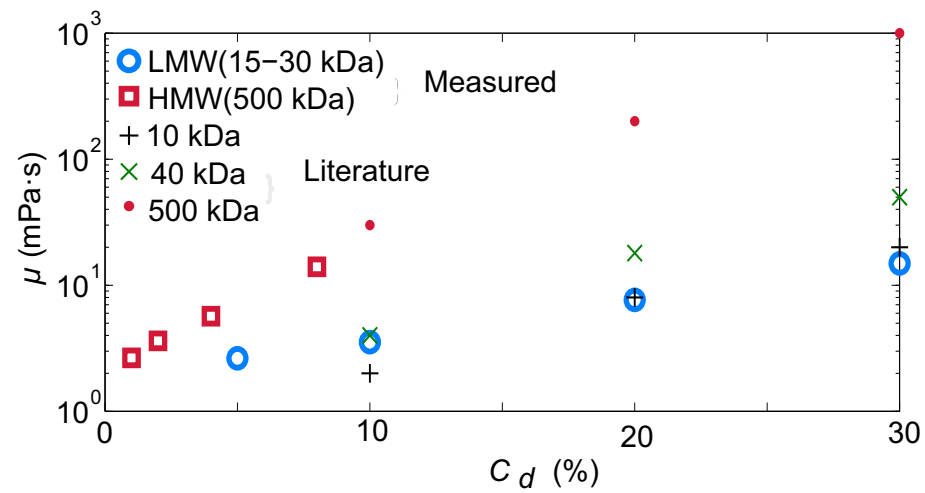

Figure S10: Measured viscosity as a function of the dextran concentration (mass \%). Literature values [47] are shown for reference, indicating reasonable agreement. 


\section{References}

[1] F. A. Navarro, M. L. Stoner, C. S. Park, J. C. Huertas, H. B. Lee, F. M. Wood, and D. P. Orgill, Sprayed keratinocyte suspensions accelerate epidermal coverage in a porcine microwound model., The Journal of Burn Care \& Rehabilitation 21, 513 (2000).

[2] D. G. Harkin, R. A. Dawson, and Z. Upton, Optimized delivery of skin keratinocytes by aerosolization and suspension in fibrin tissue adhesive., Wound Repair and Regeneration 14, 354 (2006).

[3] R. S. Kirsner, W. A. Marston, R. J. Snyder, T. D. Lee, D. I. Cargill, and H. B. Slade, Sprayapplied cell therapy with human allogeneic fibroblasts and keratinocytes for the treatment of chronic venous leg ulcers: a phase 2, multicentre, double-blind, randomised, placebo-controlled trial., Lancet 380, 977 (2012).

[4] T. S. De Windt, L. A. Vonk, J. K. Buskermolen, J. Visser, M. Karperien, R. L. A. W. Bleys, W. J. A. Dhert, and D. B. F. Saris, Arthroscopic airbrush assisted cell implantation for cartilage repair in the knee: A controlled laboratory and human cadaveric study., Osteoarthritis and Cartilage 1 (2014).

[5] S. V. Murphy and A. Atala, 3D bioprinting of tissues and organs, Nature Biotechnology 32, 773 (2014).

[6] C. Mota, D. Puppi, F. Chiellini, and E. Chiellini, Additive manufacturing techniques for the production of tissue engineering constructs., Journal of Tissue Engineering and Regenerative Medicine (2012).

[7] J. Malda, J. Visser, F. P. Melchels, T. Jüngst, W. E. Hennink, W. J. a. Dhert, J. Groll, and D. W. Hutmacher, 25th anniversary article: Engineering hydrogels for biofabrication., Advanced Materials 25, 5011 (2013).

[8] W. C. Wilson and T. Boland, Cell and organ printing 1: protein and cell printers., The anatomical record, Part A 272, 491 (2003).

[9] B. Derby, Bioprinting: inkjet printing proteins and hybrid cell-containing materials and structures, Journal of Materials Chemistry 18, 5717 (2008).

[10] K. W. Binder, A. J. Allen, J. J. Yoo, and A. Atala, Drop-on-demand ink-jet bioprinting: a primer, Gene Therapy and Regulation 06, 33 (2011).

[11] F. Guillemot, A. Souquet, S. Catros, B. Guillotin, J. Lopez, M. Faucon, B. Pippenger, R. Bareille, M. Rémy, S. Bellance, P. Chabassier, J. C. Fricain, and J. Amédée, High-throughput laser printing of cells and biomaterials for tissue engineering., Acta Biomaterialia 6, 2494 (2010).

[12] S. Moon, S. K. Hasan, Y. S. Song, F. Xu, H. O. Keles, F. Manzur, S. Mikkilineni, J. W. Hong, J. Nagatomi, E. Haeggstrom, A. Khademhosseini, and U. Demirci, Layer by layer three-dimensional tissue epitaxy by cell-laden hydrogel droplets, Tissue Engineering Part C, Methods 16, 157 (2010).

[13] A. Faulkner-Jones, S. Greenhough, J. A. King, J. Gardner, A. Courtney, and W. Shu, Development of a valve-based cell printer for the formation of human embryonic stem cell spheroid aggregates., Biofabrication 5, 015013 (2013).

[14] L. Grossin, D. Cortial, B. Saulnier, O. Félix, A. Chassepot, G. Decher, P. Netter, P. Schaaf, P. Gillet, D. Mainard, J.-C. Voegel, and N. Benkirane-Jessel, Step-by-step build-up of biologically active cell-containing stratified films aimed at tissue engineering, Advanced Materials 21, 650 (2009).

[15] J. Tritz, R. Rahouadj, N. de Isla, N. Charif, A. Pinzano, D. Mainard, D. Bensoussan, P. Netter, J.-F. Stoltz, N. Benkirane-Jessel, and C. Huselstein, Designing a three-dimensional alginate hydrogel by spraying method for cartilage tissue engineering, Soft Matter 6, 5165 (2010). 
[16] C. Fredriksson, G. Kratz, and F. Huss, Transplantation of cultured human keratinocytes in single cell suspension: a comparative in vitro study of different application techniques., Burns: Journal of the International Society for Burn Injuries 34, 212 (2008).

[17] Y. Nahmias, A. Arneja, T. T. Tower, M. J. Renn, and D. J. Odde, Cell patterning on biological gels via cell spraying through a mask., Tissue Engineering 11, 701 (2005).

[18] S. Di Risio and N. Yan, Piezoelectric Ink-Jet Printing of Horseradish Peroxidase: Effect of Ink Viscosity Modifiers on Activity, Macromolecular Rapid Communications 28, 1934 (2007).

[19] Y. Nishiyama, M. Nakamura, C. Henmi, K. Yamaguchi, S. Mochizuki, H. Nakagawa, and K. Takiura, Development of a three-dimensional bioprinter: construction of cell supporting structures using hydrogel and state-of-the-art inkjet technology., Journal of Biomechanical Engineering 131, 035001 (2009).

[20] C. J. Ferris, K. G. Gilmore, G. G. Wallace, and M. In het Panhuis, Biofabrication: An overview of the approaches used for printing of living cells, Applied Microbiology and Biotechnology 97, 4243 (2013).

[21] S. Yamaguchi, A. Ueno, Y. Akiyama, and K. Morishima, Cell patterning through inkjet printing of one cell per droplet., Biofabrication 4, 045005 (2012).

[22] R. E. Saunders, J. E. Gough, and B. Derby, Delivery of human fibroblast cells by piezoelectric drop-on-demand inkjet printing., Biomaterials 29, 193 (2008).

[23] E. Q. Li, E. K. Tan, and S. T. Thoroddsen, Piezoelectric Drop-on-Demand Inkjet Printing of Rat Fibroblast Cells: Survivability Study and Pattern Printing, ArXiv:1310.0656 (2013).

[24] A. Tirella and A. Ahluwalia, The impact of fabrication parameters and substrate stiffness in direct writing of living constructs., Biotechnology Progress 28, 1315 (2012).

[25] S. Tasoglu, G. Kaynak, A. J. Szeri, U. Demirci, and M. Muradoglu, Impact of a compound droplet on a flat surface: A model for single cell epitaxy., Physics of Fluids 22, 1 (2010).

[26] K. A. Barbee, Mechanical cell injury., Annals of the New York Academy of Sciences 1066, 67 (2005).

[27] H. Takamatsu and B. Rubinsky, Viability of deformed cells., Cryobiology 39, 243 (1999).

[28] N. Groulx, F. Boudreault, S. N. Orlov, and R. Grygorczyk, Membrane reserves and hypotonic cell swelling., The Journal of Membrane Biology 214, 43 (2006).

[29] C. Clanet, C. Béguin, D. Richard, and D. Quéré, Maximal deformation of an impacting drop, Journal of Fluid Mechanics 517, 199 (2004).

[30] C. W. Visser, Y. Tagawa, C. Sun, and D. Lohse, Microdroplet impact at very high velocity, Soft Matter 8, 10732 (2012).

[31] T. Tran, H. de Maleprade, C. Sun, and D. Lohse, Air entrainment during impact of droplets on liquid surfaces, Journal of Fluid Mechanics 726, R3 (2013).

[32] S. Mangili, C. Antonini, M. Marengo, and A. Amirfazli, Understanding the drop impact phenomenon on soft PDMS substrates, Soft Matter 8, 10045 (2012).

[33] J. Jussila, Preparing ballistic gelatine-review and proposal for a standard method., Forensic Science International 141, 91 (2004).

[34] M. A. Meyers, P.-Y. Chen, A. Y.-M. Lin, and Y. Seki, Biological materials: Structure and mechanical properties, Progress in Materials Science 53, 1 (2008).

[35] A. Allouni, R. Papini, and D. Lewis, Spray-on-skin cells in burns: a common practice with no agreed protocol., Burns: Journal of the International Society for Burn Injuries 39, 1391 (2013).

[36] T. Boland, T. Xu, B. Damon, and X. Cui, Application of inkjet printing to tissue engineering., 
Biotechnology Journal 1, 910 (2006).

[37] B. Derby, Printing and prototyping of tissues and scaffolds., Science (New York, N.Y.) 338, 921 (2012).

[38] C. Khatiwala, R. Law, B. Shepherd, S. Dorfman, and M. Csete, 3D Cell Bioprinting for Regenerative Medicine Research and Therapies, Gene Therapy and Regulation 07, 1230004 (2012).

[39] F. P. Melchels, M. A. Domingos, T. J. Klein, J. Malda, P. J. Bartolo, and D. W. Hutmacher, Additive manufacturing of tissues and organs, Progress in Polymer Science 37, 1079 (2012).

[40] T. Xu, C. A. Gregory, P. Molnar, X. Cui, S. Jalota, S. B. Bhaduri, and T. Boland, Viability and electrophysiology of neural cell structures generated by the inkjet printing method., Biomaterials 27, 3580 (2006).

[41] M. Gruene, A. Deiwick, L. Koch, S. Schlie, C. Unger, N. Hofmann, I. Bernemann, B. Glasmacher, and B. Chichkov, Laser Printing of Stem Cells for Biofabrication of Scaffold-Free Autologous Grafts., Tissue Engineering Part C, Methods 17, 79 (2011).

[42] B. Guillotin and F. Guillemot, Cell patterning technologies for organotypic tissue fabrication., Trends in Biotechnology 29, 183 (2011).

[43] Y. Lin, G. Huang, Y. Huang, T.-R. J. Tzeng, and D. Chrisey, Effect of laser fluence in laser-assisted direct writing of human colon cancer cell, Rapid Prototyping Journal 16, 202 (2010).

[44] S. Catros, B. Guillotin, M. Bačáková, J.-C. Fricain, and F. Guillemot, Effect of laser energy, substrate film thickness and bioink viscosity on viability of endothelial cells printed by LaserAssisted Bioprinting, Applied Surface Science 257, 5142 (2011).

[45] B. R. Ringeisen, H. Kim, J. A. Barron, D. B. Krizman, D. B. Chrisey, S. Jackman, R. Y. C. Auyeung, and B. J. Spargo, Laser printing of pluripotent embryonal carcinoma cells., Tissue Engineering 10, 483 (2004).

[46] F. Li, C. U. Chan, and C. D. Ohl, Yield strength of human erythrocyte membranes to impulsive stretching., Biophysical Journal 105, 872 (2013).

[47] D. Pharmacosmos, Holbaek, No Title, 2014. 


\section{8 Quantifying cell adhesion through impingement of a controlled microjet $* \dagger$}

The impingement of a submerged, liquid jet onto a cell-covered surface allows assessing cell attachment on surfaces in a straightforward and quantitative manner and in real-time, yielding valuable information on cell adhesion. However, this approach is insufficiently characterized for reliable and routine use. In this work, we both model and measure the shear stress exerted by the jet on the impingement surface in the micrometer-domain, and subsequently correlate this to jet-induced cell detachment. The measured and numerically calculated shear stress data are in good agreement with each other, and with previously published values. Real-time monitoring of the cell detachment reveals the creation of a circular cell-free area upon jet impingement, with two successive detachment regimes: (i) a dynamic regime, during which the cell-free area grows as a function of both the maximum shear stress exerted by the jet and the jet diameter; followed by (ii) a stationary regime, with no further evolution of the cell-free area. For the latter regime, which is relevant for cell adhesion strength assessment, a rela-

${ }^{*}$ Accepted for publication as: C.W. Visser, M.V. Gielen, Z. Hau, Z., S. Le Gac, D. Lohse, C. Sun, Biophysical Journal

${ }^{\dagger}$ Research design, measurements, simulations, data analysis, and article writing by CWV (all in part). 
tionship between the jet Reynolds number, the cell-free area, and the cell adhesion strength is proposed. To illustrate the capability of the technique, the adhesion strength of HeLa cervical cancer cells is determined $\left((34 \pm 14) \mathrm{N} / \mathrm{m}^{2}\right)$. Real-time visualization of cell detachment in the dynamic regime shows that cells detach either by individual-cell detachment or by collectivecell detachment (for which intact parts of the monolayer detach as cell sheets). This process is dictated by the cell monolayer density, with a typical threshold of $(1.8 \pm 0.2) \cdot 10^{9} \mathrm{cell} / \mathrm{s} / \mathrm{m}^{2}$, above which the collective behavior is mostly observed. The proposed approach presents great promises for the field of tissue engineering, as the influence of both the shear stress and the surface characteristics on cell adhesion or differentiation can be systematically studied.

\subsection{Introduction}

In the rapidly developing field of tissue regeneration, tailoring surface properties (chemistry \& structure) and growth factors are of prime importance to optimize cell adhesion to scaffolds or neighbor cells [1,2]. For instance, the cells covering the wall of engineered vascular implants need to be strong enough to withstand the shear force exerted by the blood, which is highly challenging especially for small vessels [3]. Furthermore, cell migration relates to cell adhesion, so that characterizing cell adhesion strength on a surface yields key information on their migratory capability which is essential to elucidate biological processes such as wound healing, or extravasation and intravasation in cancer biology [4]. However, due to limited availability of reliable and efficient measurement approaches, cell adhesion characteristics and their potential dependence on the shear stress are receiving relatively scarce attention.

So far, a number of methods have been proposed to apply stress on cells. Contact methods (e.g., micromanipulation [5], microcantilevers [6], or atomic force microscopy [7]) have been used to study the dynamics of single cells or small clusters of cells. However, these methods require significant investment, and, due to the limited size of the scanned area, adhesion statistics for multiple cells are cumbersome to acquire. In alternative methods for quantitative investigation of larger cell-covered surfaces, a liquid flow is applied to exert a shear stress on the cells. In that category, the technique of liquid jetting [8-14] is particularly simple and reliable. Moreover, a single measurement allows assessing the cell response to a great range of shear stresses. Finally, the detachment process can be observed in real-time, provided cells are grown on a transparent substrate. For all these reasons, jetting has been identified as the preferential method for adhesion strength measurement of biofilms [15].

In the jet impingement technique, a steady liquid jet is ejected from a submerged 
tube impacting on a cell-covered surface (see figure 8.1), resulting in cell removal in a (growing) circular area centered around the jet axis. After a certain time, the size of the cleared area reaches a plateau, characterized by the maximum cleared radius $\left(R_{\max }\right)$. From this value and that of the flow-induced stresses the adhesion strength of the cells can be calculated [9]. Despite its simplicity and widespread use [9$11,13,14]$, the impinging-jet concept has not yet been thoroughly and satisfactorily characterized from a fluid mechanics point of view [15]. Specifically, key theoretical and numerical descriptions of the shear stress are only valid for high Reynolds numbers $[16,17]$, and dedicated numerical work only provides the shear stress close to the jet exit [18,19], while conditions found experimentally lie in a broader range. Experimental validation of existing theoretical work is still lacking. Furthermore, the existence of a steady state regime is key in studies in which the cleared radius is assessed only after jet impingement $[10,13,14]$. However, it is not clear whether and, if so, this regime is reached $[9,11]$, which poses a reliability problem. Finally, cell- or biofilm removal during jet impact has received limited attention so far, while it would provide unprecedented insight into adhesion and removal processes at high shear rates, as for example used in cleaning processes [20].

In this work, and for the first time, we experimentally validate theoretical and numerical solutions of the jet-induced wall shear stress on the micro-scale, to expand our knowledge on the jet-impingement concept. Subsequently, a cell-covered surface is exposed to jet impingement, and the cell detachment characteristics are exploited to quantitatively determine the cell adhesion strength. Moreover, we benefit from the optical access provided by our device to monitor cell detachment in real time. Time-resolved observations reveal two different behaviors, where cells detach either individually or collectively as entire sheets. Altogether, our proposed approach allows not only quantifying cell adhesion strength in a straightforward manner, but also producing cell sheets, which can further be utilized for engineering tissues in a layer-by-layer manner.

\subsection{Materials and methods}

\subsubsection{Shear stress determination}

This subsection summarizes Glauert's theory, followed by our numerical and experimental methods. All cases refer to the geometry of a submerged wall jet as illustrated in figure 8.1. For theory and simulations, we assume that the fluid is of infinite depth, the flow is axially symmetric, and the length of the tube is infinite, while the side wall thickness is zero. The flow is assumed to be Newtonian, steady, laminar, and 


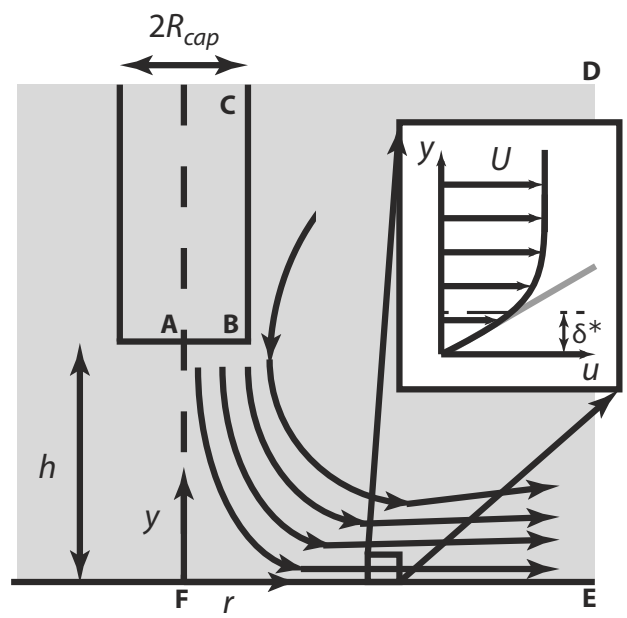

Figure 8.1: Schematic view of a submerged wall jet. The curved arrows indicate streamlines (shown only in the right half). The wall distance $h$ of the capillary exit, the radial distance $r$, and the axial distance $y$ are indicated in the figure. Capitals A to $\mathrm{F}$ are used to define the boundaries of the simulated domain. The inset shows the velocity profile $u(y)$ close to the wall and the boundary layer thickness $\delta^{*}$, which is defined by the condition $u\left(\delta^{*}\right)=0.5 U$.

incompressible. For this configuration, Glauert [16] proposed an analytical similarity solution of the flow field including the wall shear stress $\tau$, given as:

$$
\tau=\rho v\left(\frac{\partial u}{\partial y}\right)_{y=0}=\rho\left(\frac{125 F^{3}}{216 v r^{11}}\right)^{1 / 4}
$$

with $F=(1 / 8) U_{\text {cap }}^{3} R_{\text {jet }}^{4}$ the momentum flux of the incoming jet for a flat velocity profile, $u$ the horizontal velocity, $U_{\text {cap }}$ the average flow velocity at the capillary exit, $r$ the radial coordinate, $\rho$ the liquid density, $2 R_{\text {jet }}$ the inner diameter of the capillary tube, and $v$ the kinematic viscosity of the liquid. This solution is valid far from the origin and for high jet Reynolds numbers $(\operatorname{Re} \gg 1)$, defined as $\operatorname{Re}_{j e t}=2 U_{\text {cap }} R_{\text {jet }} / v$. The wall shear stress diverges as $r$ approaches the origin, where equation (8.1) no longer holds.

In the current numerical simulations the steady-state Navier-Stokes equations are solved, using COMSOL Multiphysics (Comsol Inc., Burlington, MA). The boundary conditions in the simulations are defined as follows, see figure 8.1: along the exit plane of the capillary $\mathbf{A B}$, a parabolic exit profile is used, along $\mathbf{B C}$ there is no slip, at the outflow planes $\mathbf{C D}$ and $\mathbf{D E}$ there is free outflow of fluid since $p=0$, along $\mathbf{E F}$ 
there is no slip, and on the axis of symmetry $\mathbf{A F}$ the symmetric boundary condition implies $u(r=0, y)=0$ and $\partial v / \partial y=0$. The size of the computational domain is 10 jet-diameters in width and height.

To measure the velocity field close to the surface, a bottom view micro-particle image velocimetry ( $\mu \mathrm{PIV}$ ) setup is used [21] (for details see figure S1). The working liquid is demineralized water with a $0.04 \%$ vol. concentration of red fluorescent tracer particles with a diameter of $0.52 \mu \mathrm{m}$ (Fluoro-Max, Thermo-scientific, Waltham, MA). A syringe pump (PHD 2000, Harvard apparatus, Holliston, MA) is used to generate a continuous flow. The jet is ejected from a capillary with an inner diameter of $100 \mu \mathrm{m}$ and an outer diameter of $360 \mu \mathrm{m}$ (Polymicro technologies, Molex, Lisle, IL). A microscope slide with a thickness of $150 \pm 20 \mu \mathrm{m}$ (Menzel Gläser, Braunschweig, Germany) is used as a bottom surface. To visualize the tracer particles a $100 \times$ objective (PL FLUOTAR with NA $=0.9$, Leitz, Wetzlar, Germany) is used, ensuring a sub-micrometer thickness of the measurement volume. The objective is mounted on a piezo element (NV 40/1 CLE, Piezosystem Jena, Germany) for sub-micrometer control of the measurement plane height. A dual-pulsed ND:Yag laser $(\lambda=532 \mathrm{~nm}$, Evergreen, Big Sky Laser Technologies, Bozeman, MT) is used for co-axial illumination and a dual-shutter camera (Sensicam, PCO, Kelheim, Germany) for image capturing. The timings of the laser and the camera are controlled by a pulse-delay generator (Model 575 Digital, BNC, San Rafael, CA).

\subsubsection{Cell detachment experiments}

Human cervical cancer cells (HeLa cells) are cultured in Dulbecco's Modified Eagle Medium (DMEM) supplemented with $10 \%$ (v/v) fetal bovine serum, $2 \mathrm{mM} \mathrm{L-}$ glutamine and $100 \mathrm{U} / \mathrm{ml}$ penicillin-streptomycin in an incubator, at $37^{\circ} \mathrm{C}$ with $5 \%$ $\mathrm{CO}_{2}$ concentration. To prepare the samples, the cells are seeded in Ibidi chambers without any additional chemical treatment (IbiTreat 8- or 2- well $\mu$-slides, Ibidi, Martinsried, Germany) with a density of $\sim 12,000$ cells $/ \mathrm{mm}^{2}$, and they are kept in culture for $24 \mathrm{~h}$ before the experiments. Before beginning of the experiments, the monolayer is seen to be nearly confluent and cells are stained with with calcein-acetoxymethyl ester (Invitrogen Life Technologies, Carlsbad, CA) according to the manufacturer's instructions. To assess the thickness and geometry of the monolayer, cell membranes are stained with wheat germ agglutinin (WGA-A555). Briefly, cells are fixed using paraformaldehyde (30 min, RT), washed twice with phosphate buffered saline (PBS), permeabilized with $0.1 \%$ Triton-100 (10 min, RT), washed again twice with PBS, before incubation with the staining agent $(5 \mu \mathrm{g} / \mathrm{mL})$. After thorough washing with PBS (3 times), cells are imaged using a confocal microscope (Nikon A1, using a $561 \mathrm{~nm}$ excitation wavelength). As shown in figures 8.2(b) and (c), the monolayer 
exhibits a typical height of $15 \mu \mathrm{m}$.

The cell monolayer is exposed to a wall jet schematically represented in figure 8.2(a). Capillary tubes with inner diameters of 50,100, or $250 \mu \mathrm{m}$ and an outer diameter of $360 \mu \mathrm{m}$ are aligned perpendicularly to the monolayer. The jetting liquid is PBS, which is continuously jetted at a fixed flow rate using a syringe pump (LC20AP, Shimadzu, Kyoto, Japan). To ensure laminar flow, a jet Reynolds number $\operatorname{Re}_{\text {jet }}<2300$ is used. To maintain the validity of the theoretically determined flow field for the cell-covered surface, the surface roughness needs to be small compared to the boundary layer thickness of the flow, which is estimated to be $\delta^{*} \sim \sqrt{v R_{\text {jet }} / U_{\text {jet }}}$ [22] (for details see section S.III). Automated image analysis is performed with MATLAB (The MathWorks, Natick, MA).

The cell detachment dynamics is monitored in real-time from the bottom using a camera (D5100, Nikon, Tokyo, Japan) operated at 25 frames per second and an inverted microscope (Zeiss CFL40, Oberkochen, Germany). The magnification of the objective $(5 \times, 10 \times$ or $20 \times$, Olympus, Tokyo, Japan) is chosen to match the cleared radius of the cell layer. The cell layer is co-axially illuminated from below using a white light source (LS-M352, Sumita, Saitama-City, Japan) and a filter set (Zeiss filter set 09) for fluorescent imaging of the calcein-stained cells.

\subsection{Results}

\subsubsection{Shear stress}

Figure 8.3(a) shows our experimental and numerical results together with the analytical solution of equation (8.1), as already reported in [16]. The shear stress exhibits a maximum at a radial distance $r \approx R_{\text {jet }}$, and strongly decays for larger diameters. Close to the origin, the large vertical velocity component results in a strong outof-plane motion of the tracer particles. Therefore, the $\mu$ PIV measurements are less reliable for $r<R_{j e t}$ and are represented in low contrast in figure 8.3(a) (see section S.I for details). For $r>R_{j e t}$, our experimental results agree well with the theory and simulations. It should be noted that the experimental errors are relatively large, which is due to uncertainties of the measurement height and averaging of the radial velocity over some radius increment.

Figure 8.3(b) compares our results to Glauert's solution (equation 8.1) as well as to numerical work by Deshpande and Vaishnav [18]. Excellent agreement is found between our simulations and Glauert's theory, already for $r \gtrsim 1.5 R_{j e t}$. Similarly, the agreement between our simulations and the numerical solution proposed by Deshpande and Vaishnav [18] is good even if a small discrepancy is found, probably due 

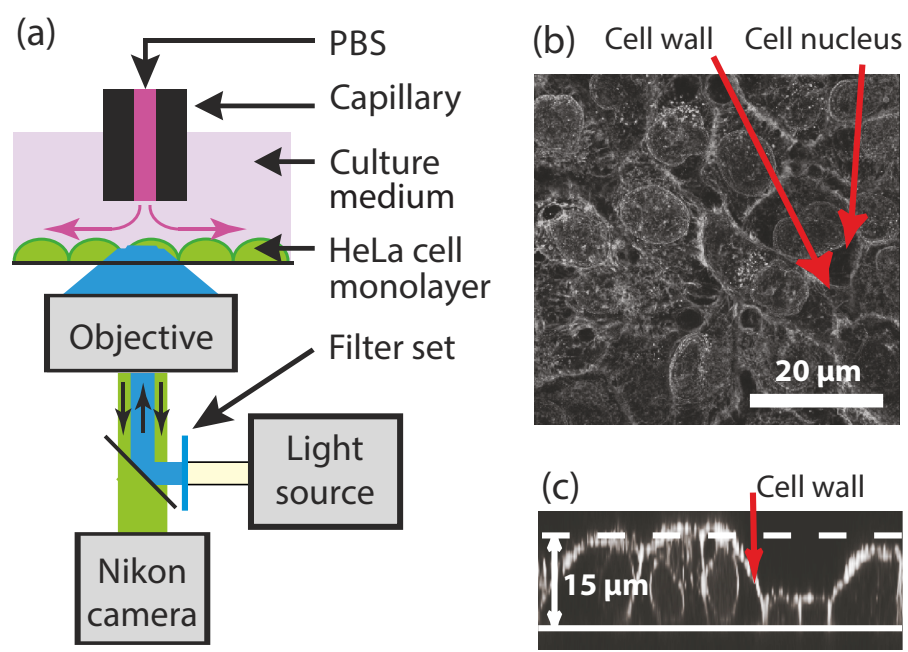

Figure 8.2: (a) Scheme of the setup used to measure cell detachment. A submerged liquid jet impacts on a cell monolayer attached to a glass surface. The monolayer is illuminated and visualized from below. (b) In-plane and (c) side-view confocal microscope image of a monolayer of fixed HeLa cells stained with WGA, at the same scale. The cell walls and cell nucleus walls are visualized, by the absorption of a fluorescent marker. The solid line in the bottom in (c) indicates the glass surface and the dashed line in the top gives an indication of the typical thickness of the cell layer, which is about $15 \mu \mathrm{m}$.

to differences in the boundary conditions along CD and DE (figure 8.1): We use free outflow boundary conditions with $p=0$, whereas Deshpande and Vaishnav [18] use constraints for the stream function and vorticity (which is not available in the COMSOL solver used here), to model the vanishing flow velocities far from the origin.

The main limitations of the current method are that the numerical results deviate from Glauert's result for $r>10 R_{\text {jet }}$ (see section S.II), and the rough cell monolayer which might influence the shear stress for a part of our measurements (see section S.III). In the rest of this article we use Glauert's solution to analyze cell detachment in far field (where the cell-free area exceeds $3 R_{j e t}$ ), and our numerical results in the near field (where the peak shear stress is the key control parameter).

\subsubsection{Cell detachment measurements}

Figure 8.4 shows a time series of the cell monolayer removal due to the impinging jet. After starting the jet (at $t=0 \mathrm{~s}$ ), a growing cell-free area is observed. The well-defined edge of this area is assessed by automated image analysis and manually 

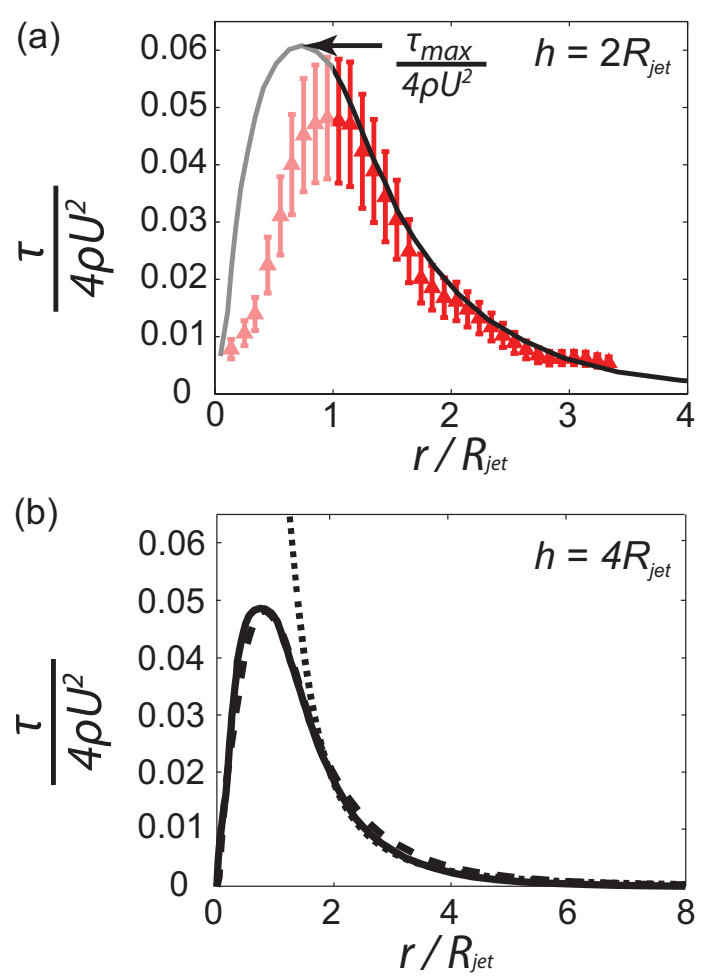

Figure 8.3: Non-dimensional wall shear stress versus non-dimensional radius for $\mathrm{Re}_{\text {jet }}=$ 100. (a) The markers represent our $\mu \mathrm{PIV}$-measurements and the solid line represents our steady-state Navier-Stokes simulations, for $h=2 R_{\text {jet }}$. The pale markers for $r / R_{\text {jet }}<1$ have a larger measurement error than indicated, due to out-of-plane motion of tracer particles. (b) The solid line represents our steady-state Navier-Stokes simulations for $h=4 R_{\text {jet }}$. The dashed line represents steady-state Navier-Stokes simulations by Deshpande and Vaishnav [18]. The dotted line represents Glauert's theoretical solution [16]. 

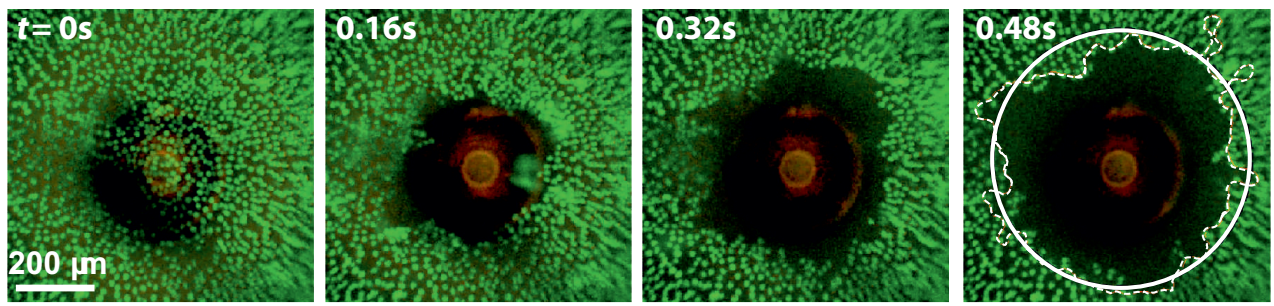

Figure 8.4: Bottom view of the HeLa cell monolayer during jet exposure. The cells are stained with calcein (green) for visualization purposes. The (red) circular end of the capillary is seen in the center of each image; around this position the cells start to detach after jetting is started $(t=0 \mathrm{~s})$. The dark area in the first frame is caused by poor visualization of the cells in that region. The subsequent images show the growth of the cleared area as a function of time. The last image illustrates the detected edge of the detached area (white dashed line) and a circular fit of this interfacial curve (white solid line), which is determined for each frame. The supplementary material contains the full movie of this experiment.

checked. A circle is fitted to the radial position where $50 \%$ cell detachment is observed. Repeating this procedure for each image subsequently provides the cleared radius as a function of time, as shown in figure 8.5(a). Initially, in the "dynamic regime" (I), the cleared radius is increasing *; all frames in figure 8.4 belong to this regime. After some time a "stationary regime" (II) is reached when no more cells are detached and the cleared radius reaches a plateau. To quantify the time-dependence of the radius of the cleared area $R(t)$ and determine the time after which the stationary regime is reached, the individual data sets are fitted by the following expression:

$$
R(t)=R_{\max }\left(1-e^{-t / t^{*}}\right)
$$

where $R_{\max }$ and $t^{*}$ represent the maximum cleared radius and the detachment time scale, respectively. Figure 8.5 (b) shows the variations of the cleared radius as a function of time, after normalization by $R_{\max }$ and $t^{*}$, respectively. The temporal-axis is plotted for $t \leq 10 t^{*}$, as the cleared radius does not increase any further at later times. The discrepancy between the fit and the measurements can be explained by the complex dependence of the shear stress on the radial position (as already discussed in section 8.3.1). Nevertheless, equation 8.2 reasonably matches our data and directly provides the order of magnitude of the detachment time- and length-scales $\left(t^{*}\right.$ and

\footnotetext{
¥Occasionally, some cells remain attached to the surface in the center of the cleared radius, where the shear stress is low (see figure 8.3). This corresponds to observations in ref. [13]. However, as the cell diameter and the initial jet diameter are of similar size, the shear stress in the center cannot be uniquely defined. Therefore, this aspect was not included in the discussion
} 
$R_{\max }$, respectively). For example, a typical growth rate of $R_{\max } / t^{*}$ can be readily defined, and is discussed in section S.IV. Figure 8.6, where $t^{*}$ is plotted as a function of the Reynolds number and the jet diameter, indicates that $t^{*}<2.5 \mathrm{~s}$ for all our measurements. Therefore, for the current control parameters, the steady state is always reached within $25 \mathrm{~s}$.

\subsubsection{Adhesion strength assessment}

Figure 8.7 (a) represents the fraction of the remaining adherent cells as a function of the radial position in the stationary regime. All detachment takes place in a narrow radial region, which is consistent with figure 8.4. To determine the cell adhesion strength, first the shear stress is determined as a function of the radial position, using equation (8.1). Following this, the fraction of adherent cells as a function of the shear stress is plotted (see figure 8.7(b)). The cell adhesion strength, which is defined as the shear stress where $50 \%$ of the cells is detached from the surface [23], is readily determined from this graph. From the present experiments, where HeLa cells are grown on a surface without any additional coating, we evaluate the cell adhesion strength from each measurement (with different capillary diameters and Reynolds numbers). Taking the median of the values obtained provides a cell adhesion strength $\tau_{c}=(34 \pm 13) \mathrm{N} / \mathrm{m}^{2}$, where the error indicates the standard deviation. Interestingly, the detachment regime, as discussed in section 8.3.4 and indicated with colors in figure 8.7(b), does not significantly affect the adhesion strength.

Figure 8.7(a) indicates a strong dependence of the cleared radius on the Reynolds number (indicated with colors) and a weak influence of the jet radius (indicated by the markers). This is due to the radial dependence of the shear stress. By inserting $\tau_{c}$ back into equation (8.1), the cleared radius is theoretically derived as a function of the Reynolds number. The results are represented in figure 8.8, as solid lines for each capillary diameter used. The indicated error bar is assumed from the uncertainty in $\tau_{c}$, and it is comparable for the other capillary diameters. Overall, good agreement is found with our measurements for all capillary diameters, confirming that this method is robust with respect to the flow velocity and the capillary size, and it explains the strong Reynolds-number dependence of the cleared radius.

Below a certain threshold Reynolds number, usually no cell detachment is observed (indicated by markers on the $x$-axis of figure 8.8), since detachment is expected only if the maximum shear stress exerted by the flow $\left(\tau_{\max }\right)$ exceeds the cell detachment threshold $\tau_{c}$. Therefore, determination of the threshold Reynolds number allows for independent determination of the shear stress. Since the Glauert solution does not provide the maximum shear stress, the numerical results are used to provide this parameter as a function of the Reynolds number, as shown in figure S3. Equating 
(a)

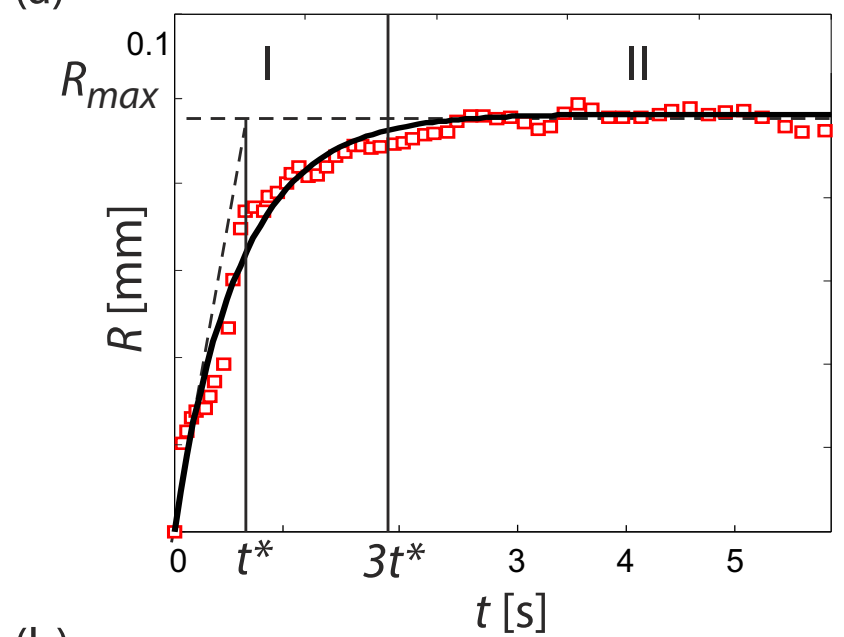

(b)

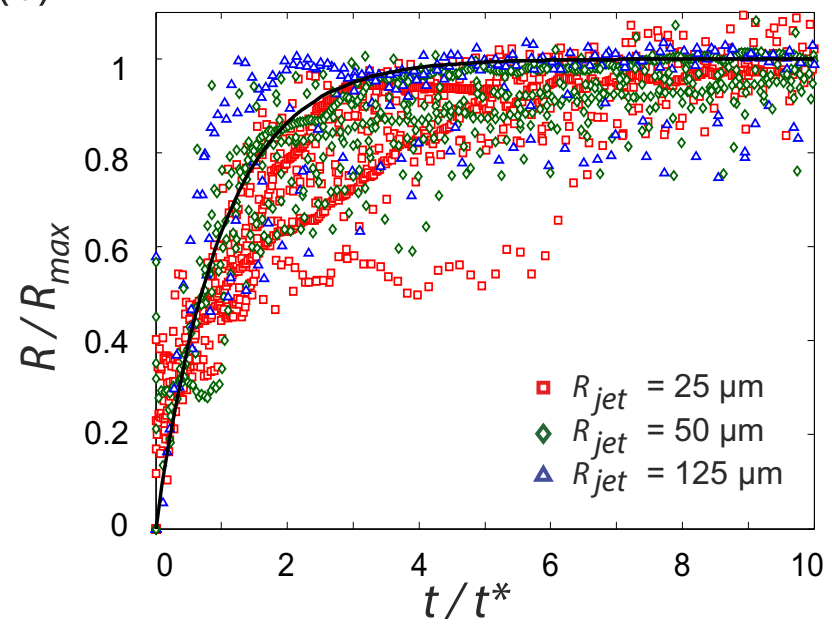

Figure 8.5: (a) Measurement of the cleared radius $R(t)$ versus time $t$, for $\operatorname{Re}_{j e t}=85$ and $R_{j e t}=25 \mu \mathrm{m}$. The thick solid line indicates the fit function (equation (8.2)) describing the experimental data. The horizontal dashed line indicates the maximum cleared radius $R_{\max }$ and the tilted dashed line the initial slope of the fit function. Their intersection defines the detachment time constant $t^{*}$. Two regimes are identified: the dynamic regime (I) for $t<3 t^{*}$ and the stationary regime (II) for $t>3 t^{*}$, according to our definition. The solid line indicates the separation between the regimes. (b) All measurements of the cleared radius versus time after normalization by $R_{\max }$ and $t^{*}$, respectively, for different $R_{\text {jet }}$. The fit function is shown as a black line. 


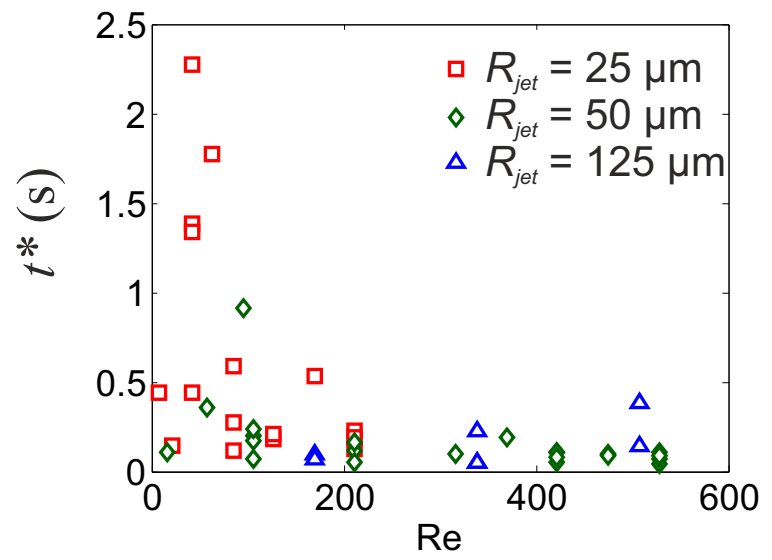

Figure 8.6: Detachment time scale $t^{*}$ versus the Reynolds number, for different capillary diameters.

this maximum shear stress to $\tau_{c}$ readily provides the critical Reynolds number below which no cell detachment is expected as $\operatorname{Re}_{j e t, c}=\operatorname{Re}_{j e t}\left(\tau_{\max }=\tau_{c}\right)$, or:

$$
\operatorname{Re}_{j e t, c}=\frac{R_{j e t}}{v} \sqrt{\frac{\tau_{\max }}{4 \rho}} .
$$

For the capillary diameters used in the present study, $\operatorname{Re}_{j e t, c}$ is indicated by the dashed lines in figure 8.8. For $\mathrm{Re} \geq \operatorname{Re}_{j e t, c}$, cell detachment is expected, and it is always observed. No cell detachment is expected for $\operatorname{Re} \leq \operatorname{Re}_{j e t, c}$, as observed in the measurements with the $125 \mu \mathrm{m}$-capillary tube. For smaller capillary tubes, a finite-area cleared radius below the threshold is observed. Here, the cell size is of the same order as $R_{\text {jet }}$ and $h$. Therefore the cells may significantly influence the flow field, resulting in unexpected cell removal as observed.

Altogether, the cell adhesion strength can be obtained from measuring either the fraction of adherent cells as a function of the radius (figure 8.7) or the threshold Reynolds number (dashed lines in figure 8.8) in the stationary regime (subsection 8.3.2). The former method has the advantages that it also provides the distribution of the adhesion strength, that it is less susceptible to outliers, and that it is common practice already for radial flow chambers [23, 24]. Therefore, this method is recommended for future measurements with the jet impingement method. 

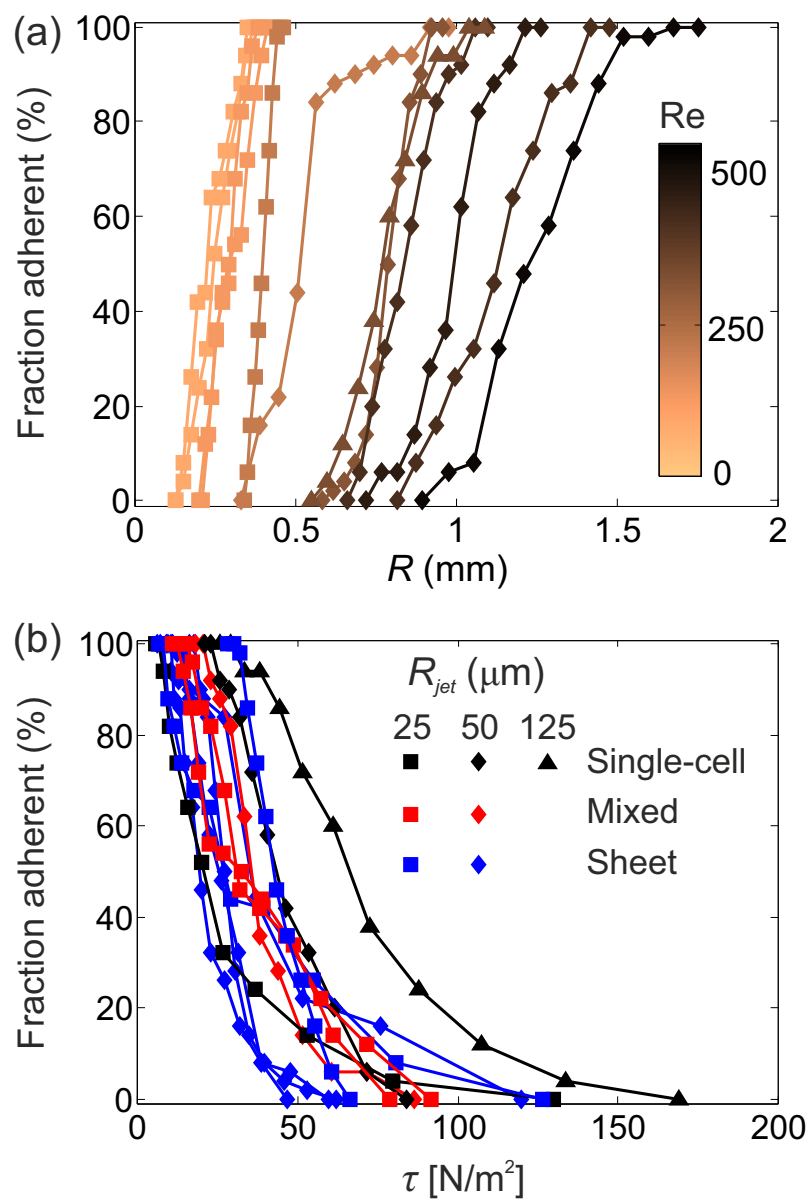

Figure 8.7: Fraction of adherent cells in the steady state. Figure (a) shows the dependence on the radius; the colors indicate the Reynolds number. Figure (b) shows the dependence on the shear stress. The color indicates the detachment regime (which not significantly affects the fraction of adherent cells). Black: single cell detachment (see figures 8.4 and 8.9), blue: sheet detachment (see figure 8.10), red: mixed cases. In both subplots the marker shape indicates the jet radius. 


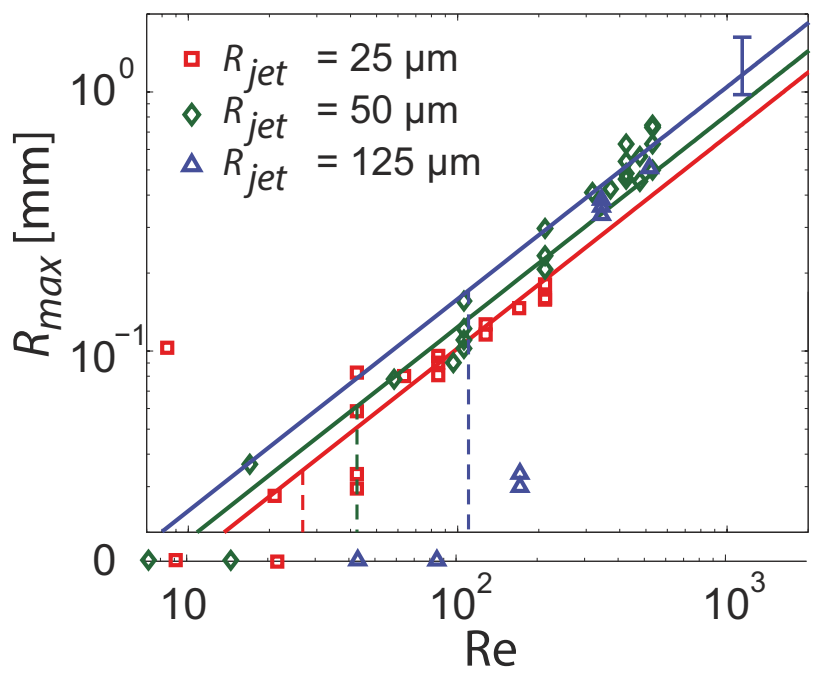

Figure 8.8: Maximum cleared radius versus the Reynolds number, for different capillary diameters. The symbols show the measured values (points on the $x$-axis indicate $R_{\max }=0$, i.e. no cell detachment). The solid lines give the solution of the theory for cell detachment, the dashed lines indicate $\operatorname{Re}_{c}$ (i.e. $\tau_{\max }=\tau_{c}$ ) beyond which cell detachment is expected. The error bar is indicative for all measurements.

\subsubsection{Cell detachment behavior in the dynamic regime}

Next to the quantification of cell adhesion, the jet impingement device proves to be a powerful tool to monitor cell detachment in real-time. Specifically, the existence of two distinct detachment processes is revealed: either following cell by cell removal (or single cell detachment), as illustrated in figures 8.4 and 8.9, or removal of cell sheets (or collective detachment), as illustrated in figure 8.10. These different regimes are discussed in more detail in the following.

First, the cell-by-cell removal mechanism (figure 8.9(a)) is observed in many experiments. This behavior is mostly found for non-confluent monolayers, where cells adhere separately on the surface without establishing any cell-cell contact. In that configuration, cell adhesion is solely governed by the interactions between the cells and the surface. These interactions are expected to be weak since no dedicated coating (e.g., fibronectin or any other extracellular matrix protein) is applied prior to experiments [24-26]. In other experiments or in some areas in the culture dish, cells form a more tight and confluent monolayer, where they are likely to develop not only cell-substrate interactions but also cell-cell contacts. In general, such a dense configuration would be observed if cells are given more time to adhere and proliferate 


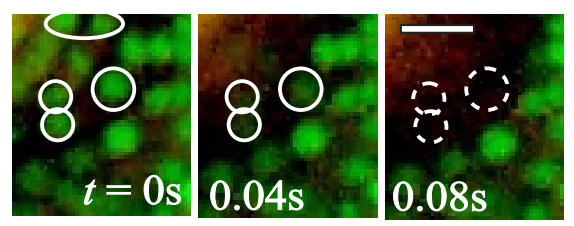

Figure 8.9: Illustration of single-cell poration and detachment. White circles indicate the cell location and white dashed circles indicate the detachment location of the cell. The circled cells become less bright due to loss of the fluorescent marker by cell poration. The oval indicates two cells that are not visible on the subsequent frame, and assumed to be totally removed immediately. The scale bar indicates $50 \mu \mathrm{m}$.

on the surface, or if a higher cell density is employed upon seeding [24-27]. In our experiments, these parameters (cell seeding density and time between cell seeding and detachment experiments) are kept constant, but both configurations (single cell adhesion or monolayer formation) are concomitantly observed. In terms of cell detachment, dense monolayers give rise to another removal process, where entire sheets of cells are detached - or collective detachment (figure 8.10). Since the cells retain their green fluorescence during detachment (see figure 8.10), it is assumed that the cell membranes remain intact, that cells are entirely removed from the surface, and that they remain viable.

Altogether, these experiments suggest that the cell density governs the threshold between the single cell detachment regime and the sheet detachment regime. In figure 8.11 the detachment regime is plotted as a function of the cell density and the Reynolds number. For cell densities exceeding $(1.8 \pm 0.2) \cdot 10^{9}$ cells $/ \mathrm{m}^{2}$ (indicated by the dashed line in figure 8.11), sheet detachment is observed for all Reynolds numbers. In other words, at higher densities cell-cell interactions seem to be dominant in comparison to cell-substrate interactions. This is to be expected, since protein-protein interactions between the cells are stronger than those established with the substrate, which are purely electrostatic here since no dedicated coating is applied.

Finally, in some experiments in the single-cell detachment regime, a gradual decrease in the intensity of the fluorescent marker loaded in the cells before experiments (calcein) (See circled cells in figure 8.9(a)). This seems to indicate that the shear stress exerted on the cells gives rise to poration of the cell membrane, leading to progressive release of calcein out of the cells and occasional cell death at the edge of the cleared area (see figure S7). Vice versa, membrane poration can be used for medicine delivery into cells, as achieved by cavitation bubble implosion next to a cell monolayer [17, 28, 29]. Therefore, our results indicate that shear-induced medicine delivery could be an interesting application of the jet impingement method. 


\subsection{Discussion}

The currently employed jet-impingement method is now compared to alternative flow-based methods to assess cell adhesion characteristics. The key difference between the different approaches lies in the temporal and spatial homogeneity of the shear stress exerted to the cells. The most spatially and temporally homogeneous flows are provided by a Taylor-Couette setup [30], a cone-and-plate shearing apparatus [31], or microfluidic devices [32]. In these devices, all cells are exposed to an equal shear stress, which means that individual measurements are required for each shear stress value. Therefore, these approaches are primarily interesting if a homogeneous shear stress is required. Temporally homogeneous but spatially inhomogeneous flows are provided by the herein presented impinging-jet method, or by radial flow chambers $[20,23,24,33]$. While a radial flow chamber has a top plate at a fixed position from the cell-covered wall (which confines the flow), the jetimpingement device has a free surface. In both devices a liquid jet impacts on the surface, and decreasing shear stress values are found for increasing radial positions, so that the cell response to a large range of shear stresses can be studied in a fast and reproducible manner, and for large numbers of cells. Alternatively, temporally and spatially inhomogeneous shear stresses are observed in cavitation bubble implosion $[17,28,34,35]$, which also gives rise to the impact of a jet on the surface. However, the duration of these jets is limited to a time scale in the ms-range, and they have characteristic impact velocities of $\sim 100 \mathrm{~m} / \mathrm{s}$. The shear stress developed by these pulsed jets is unknown, since the flow characteristics are hardly accessible either experimentally, numerically, or theoretically. Therefore, cavitation bubble implosion is primarily suitable for assessment of the cells after the impact of the jet, to for example study jet-induced drug delivery [34].

For the closely related methods of jet impingement and radial flow chambers, major differences exist. For instance, we consistently observe an expanding, roughly circular cell-free area in our experiments (figure 8.4), with a relatively sharp and welldefined border between the monolayer and the cell-free area. This pattern deviates from the gradual cell removal reported in radial flow chambers [23], which can be explained by the different flow geometries. In the impinging jet method, the shear stress decays much faster (scaling as $\tau \sim r^{-11 / 4}$ ) than in the radial flow-cell geometry (where $\tau$ decays as $\tau \sim r^{-1}$ ). When assuming a similar band-width of detachment shear stresses (see figure 8.7(b)), our flow profile naturally results in a narrow detachment region. Another key difference is the earlier onset of the validity of the analytical solution describing the shear stress for known Reynolds numbers (microjet: $r>2 R_{\text {jet }}$ for $\operatorname{Re}=100$; radial flow chamber: $r>4 R_{j e t}$ [23]). Therefore, the jet-impingement technique is more appropriate if high shear rates must be tested, as 

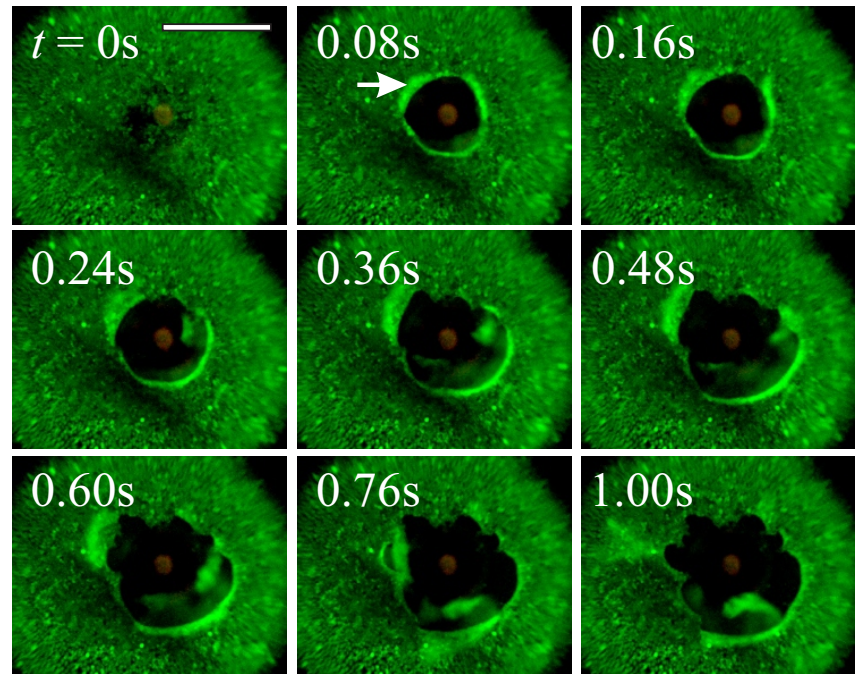

Figure 8.10: Bottom view of the HeLa cell monolayer during jet exposure, illustrating the sheet-detachment regime. The scale bar indicates $0.5 \mathrm{~mm}$. The arrow indicates the initial location of a detaching cell sheet. Subsequent frames show the ongoing growth of the sheet until detachment.

encountered for example in cleaning applications [20]. Finally, all elements composing for the jet impingement concept are commercially available and the setup can be constructed in a few hours, whereas other flow systems are usually custom-made. For all these reasons, the jet impingement concept is particularly appropriate for routine study of cell detachment.

We envision that the jet impingement approach could be applied as a screening methodology to identify optimal surface properties in the field of tissue regeneration by systematically characterizing the cell adhesion strength on a great variety of surfaces. Of particular interest is the evaluation of cell adhesion strength under continuous flow conditions, as found for instance in engineered vascular implant $[3,36]$. Typically, cell delamination is observed after a while due to the blood stream and due to the too high shear stress. As a consequence, the engineering of vascular implants with diameters below $6 \mathrm{~mm}$ is still highly challenging, while of key importance for treatment of cardiovascular diseases [37, 38]. Since the herein proposed approach allows testing a wide range of shear stress conditions in one single experiment, it would help optimizing surface properties for cell adhesion, and eventually decrease the diameter of the synthetic implants.

An interesting and unexpected result is that cells can detach collectively to form 


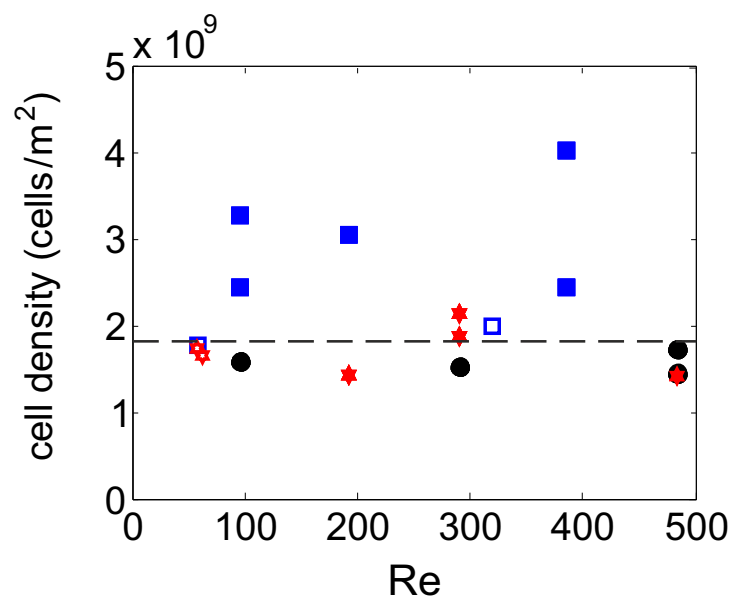

Figure 8.11: Detachment regime as a function of the Reynolds number and the cell density. Single-cell detachment, sheet detachment, and mixed-detachment measurements are indicated by black circles, blue squares, and red stars, respectively. Filled markers are obtained for $R_{\text {jet }}=100 \mu \mathrm{m}$, open markers correspond to $R_{\text {jet }}=25 \mu \mathrm{m}$. The dashed line indicates the cell density separating the single-cell detachment and sheet detachment regimes.

cell sheets upon exposure to the jet [39], provided the initial monolayer is dense enough and cell-cell junctions are established. It would be of great interest to further study this phenomenon, as current technologies for cell sheet detachment are relatively slow [39, 40] or require highly sophisticated surfaces [41]. For instance, one could vary the surface properties for instance to limit cell adhesion and strengthen cell-cell interactions for the release of large sheets of viable cells that could subsequently be utilized to form microtissues using a layer-by-layer assembly approach. For that particular application, cell viability after exposure to the jet should be carefully be examined, an aspect which has not been studied in detail here since, after their release, the cell sheets could not be tracked in solution.

\subsection{Conclusions}

Microjet impingement provides a simple and reliable concept for cell adhesion strength measurements and real-time observations of the detaching cells. We experimentally and numerically characterize the flow, measure the cell adhesion strength, and use a real-time view to study different cell detachment regimes.

The first measurements and new numerical simulations of the shear stress exerted 
by jet impingement in the micro-scale domain are presented. For high Reynolds numbers and far from the symmetry axis, the solution proposed by Glauert [16] is confirmed. This solution is particularly valid as close as $r>2 R_{\text {jet }}$ (for $\mathrm{Re}=100$, figure 8.3). For low Reynolds numbers and close to the origin, our numerical model confirms and extends the results reported by Deshpande and Vaishnav [18, 19].

Real-time observations of the cell monolayer detachment reveal a dynamic regime, in which the cell-free area continuously increases, followed by a stationary regime where no more cells are detached. The development of cell-free radius is fitted as $R(t)=R_{\max }\left(1-e^{-t / t^{*}}\right)$, where $t^{*}$ represents the time scale for the dynamic regime and $R_{\max }$ describes the final radius. The stationary regime allows for determination of the cell adhesion strength, for which procedure guidelines for consistent and reliable measurements are proposed. Following these, the adhesion strength of HeLa cells is obtained as $(34 \pm 14) \mathrm{N} / \mathrm{m}^{2}$. In the dynamic regime, the real-time view of the monolayer reveals two characteristic detachment regimes: single cell detachment for low-density monolayers and sheet or collective detachment for more dense monolayers. The threshold cell density between these two regimes is assessed to be $(1.8 \pm 0.2) \cdot 10^{9}$ cells $/ \mathrm{m}^{2}$, and is independent of the Reynolds number.

The jet impingement technique opens new avenues in the field of tissue regeneration not only to measure cell adhesion strength towards optimization of biomaterial properties, but also as a tool to produce cell sheets that can be used to generate microtissues in a controlled self-assembly approach. 


\subsection{Supplementary material}

\section{S.I. Experimental determination of the shear stress}

The shear stress $\tau$ on a liquid-solid interface is given by the velocity gradient at the wall,

$$
\tau=\left.\rho \nu \frac{\partial u}{\partial y}\right|_{y=0},
$$

where $y$ is the distance from the wall, $u$ is the flow velocity tangential to the wall, $v$ the kinematic viscosity and $\rho$ is the liquid density. It can thus be obtained by measuring $u$ and taking the derivative in the constant-shear region close to the wall. The laminar boundary layer thickness for axisymmetric stagnation-point flow can be obtained from the textbook by Schlichting \& Gersten (2000) [22]. Close to the wall, the region with constant shear rate exists within the $50 \%$-boundary layer, where $u(r, y)<0.5 U(r)$ with $U(r)$ the far-field flow velocity. Using dimensional analysis, we estimate the thickness of this boundary layer as $\delta^{*} \sim \sqrt{v R_{\text {jet }} / U_{\text {jet }}}$. This results in a minimum boundary layer thickness of $\delta^{*} \approx 4 \mu \mathrm{m}$ for $R_{j e t}=25 \mu \mathrm{m}$ and $\operatorname{Re}_{j e t}$ $=250$. Therefore, from horizontal velocity-field measurements at wall distances $y<$ $\delta^{*} \approx 7 \mu \mathrm{m}$ we can directly derive the shear stress.

For the (standard) $\mu \mathrm{PIV}$ technique employed here [21] (see figure S1), the basic principle is that the tracer particles move with the flow and the frame-to-frame time is known, thus the flow velocity can be obtained from the particle displacement. First a recording of at least 140 image pairs is made, showing the tracer particles. For each image, structures much smaller or larger than a single tracer particle are removed using a band pass filter, i.e. only single tracer particles in the focal plane remain visible. Then, for a each individual pixel, the value with the lowest light intensity of all images is obtained. This results in a minimum-intensity image, which was subtracted from all images. The resulting images allow for excellent visualization of the particles in the measurement plane, as shown in the inset of figure S2(a). From this, velocity vectors are obtained by dividing the images into regions and crosscorrelating these regions for each image pair. As the flow is steady, the correlations are then averaged to obtain a single, more reliable velocity vector. A multi-grid approach (making the correlation windows smaller in subsequent correlations) provides a high-resolution velocity field, as shown in figure S2(a). Vectors within a radial increment $d r$ (typically, $d r=10 \mu \mathrm{m}$ ) are averaged to obtain a unique velocity value for each radial distance. Similar analyses were performed at measurement planes at different heights above the surface, resulting in figure S2(b). Measurements closer than $2 \mu \mathrm{m}$ to the surface were not used, as there the $\mu \mathrm{PIV}$ technique is not reliable because then the particle diameter is similar to the patricle-surface distance, and as the 


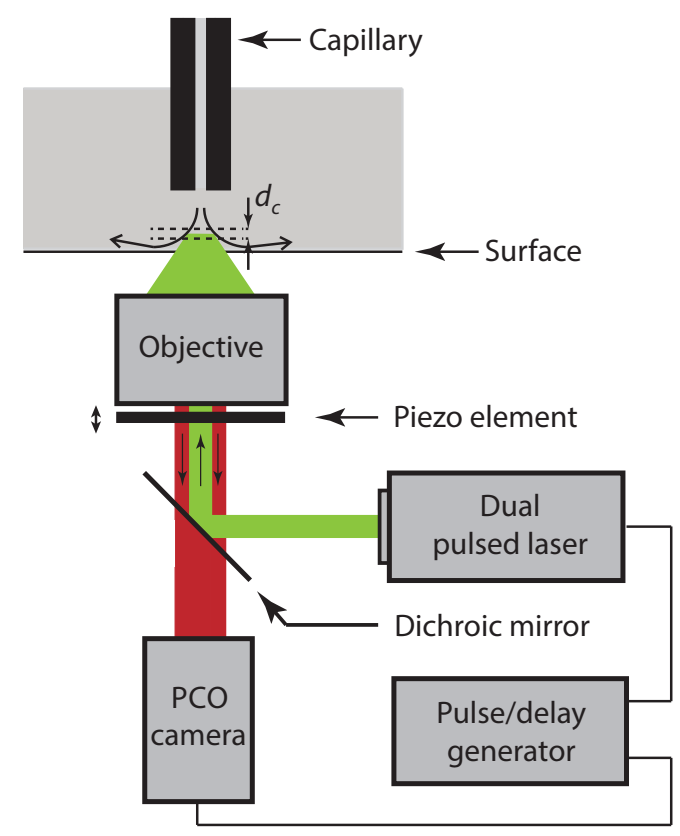

Figure S1: Schematic view of the setup for $\mu$ PIV measurements. $d_{c}$ is the thickness of the correlation plane.

error of the measurement plane height is $\sim 1 \mu \mathrm{m}$. At the surface, a no-slip boundary condition is assumed.

With the velocity known at radial positions at different heights, the wall shear stress is calculated according to equation (8.4): $\tau(r)=\rho v \partial u /\left.\partial y\right|_{y=0} \approx \rho v u(y, r) / y$. The result is shown in figure 8.2. To allow for direct comparison to numerical and literature values, it is normalized by $4 \rho U^{2}$ and the radius is normalized by $R_{j e t}$. The errors are due to uncertainties of the measurement height and averaging of the radial velocity over the radial increment $d r$. Close to the origin, the large vertical velocity component results in a strong out-of-plane motion of the tracer particles, i.e. the PIV measurements are not reliable for $r<R_{j e t}$ and are represented in low contrast.

\section{S.II. Numerical analysis of the shear stress}

New simulations were performed mainly to determine the shear stress in the whole flow field. Here we discuss in more detail the comparison with Glauert's solution (in the far field) and previous simulations $[18,19]$ (in the near field).

The maximum shear stress (for given Reynolds number) is compared to previous work in figure $\mathrm{S} 3$, as this parameter is used for our analysis of the dynamic regime. 
(a)

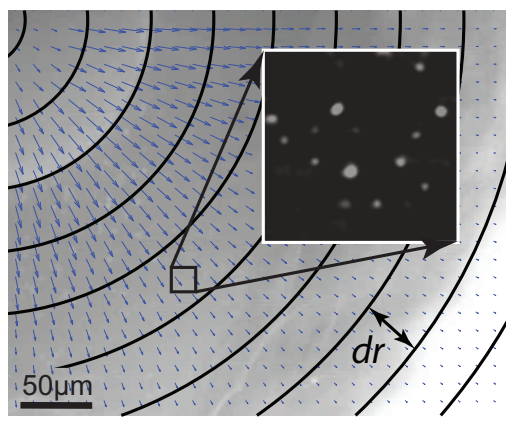

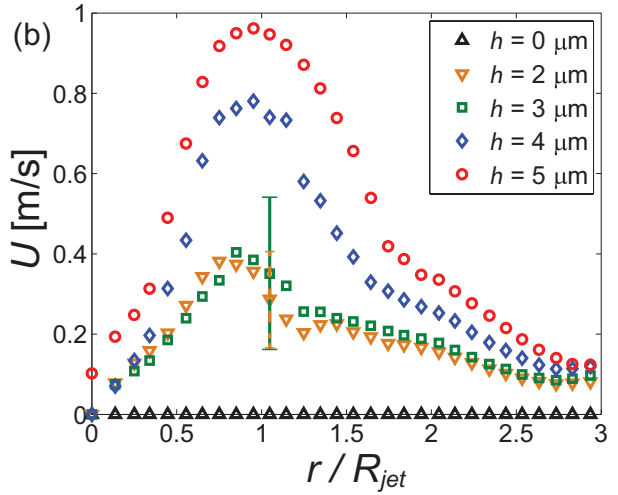

Figure S2: Velocity measurements for $\operatorname{Re}_{j e t}=100, R_{j e t}=50 \mu \mathrm{m}$, and $h=2 R_{j e t}$. (a) Bottom view at $y=4 \mu \mathrm{m}$ above the surface. The velocity vectors are plotted as arrows. The black lines show example edges of bins used to obtain the radial velocity versus the radial position. The inset shows the particles used for tracking after image enhancement. (b) Radial velocity versus dimensionless radial distance $r / R_{\text {jet }}$. Measurements are performed at $y=2$ to $5 \mu \mathrm{m}$ above the surface. At the surface a no-slip boundary condition is imposed (black triangles). The error bars represent the maximum error for all measurements (explained in the text).

Good agreement is found. In addition, the far-field shear stress is compared this to Glauert's solution (equation (8.1)) in figure S4. Here, agreement was poor even after an extension of the domain and adjustement of the grid. Only after performing similar calculations with Fluent (Ansys, Pittsburgh, PA) and extending the radial domain size to $40 R_{\text {jet }}$, quantitative agreement with Glauert's solution could be obtained for $\operatorname{Re}_{j e t} \geq 1000$ (see figure S5). However, here the near-field maximum shear stress is inconsistent with figure S3, despite several simulation attempts. A direct comparison of the far-field shear stress to related numerical work [17-19, 35] is not feasible, since there the shear stress at large radial distances is not provided. In conclusion, we recovered Glauert's solution for $\operatorname{Re}>1000$, using the Fluent package

Determination of the wall shear stress as a function of the Reynolds number (for $1<\operatorname{Re}_{j e t}<1000$ ) and the radial position would allow to find the onset of the validity of Glauert's solution, which would be highly useful for applications. However, it is beyond the scope of this work due to excessive computational costs. Therefore, we use Glauert's solution to analyze cell detachment in the stationary regime (as $R_{\max } / R_{\text {jet }}>3$ ), and the Comsol results for the analysis of the dynamic regime (where the peak shear stress is the key control parameter).

\section{S.III. Influence of the boundary layer on cell adhesion measurements}




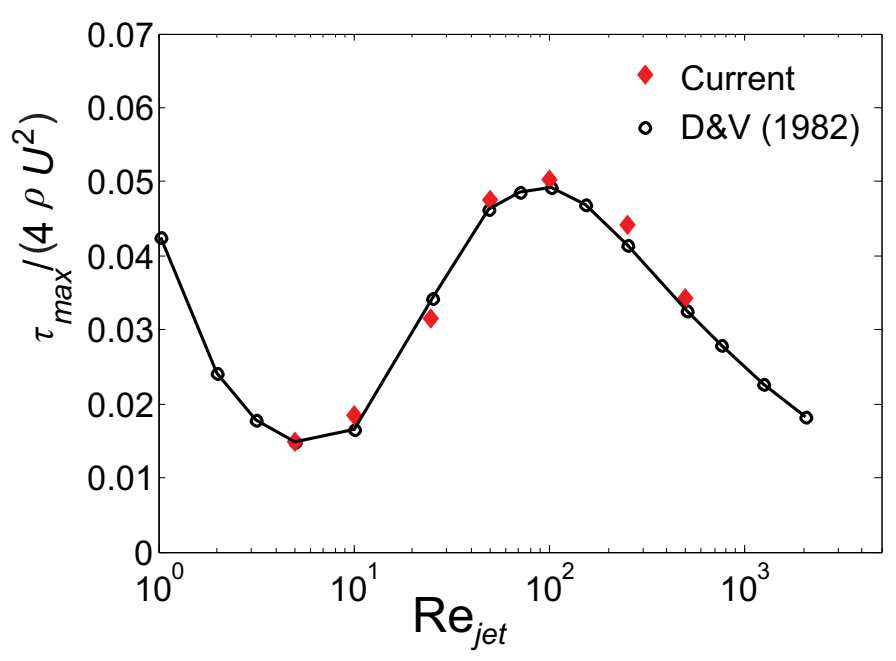

Figure S3: Maximum shear stress versus the jet Reynolds number. Results from current and previous [18] numerical work are shown.

As discussed in section S.I, a minimum boundary layer thickness of $\delta^{*} \approx 4 \mu \mathrm{m}$ is estimated for our experiments, which is comparable to the roughness of the cell monolayer (estimated as $R_{a}=5 \pm 5 \mu \mathrm{m}$ from figure 3(c) of the main document). Therefore, the cell monolayer may significantly influence the boundary layer flow and the resulting local shear stress.

Much thicker boundary layers are estimated for the larger capillary tubes used, for example $\delta^{*} \sim 20 \mu \mathrm{m}$ is found for $R_{\text {jet }}=125 \mu \mathrm{m}$ and $\mathrm{Re}=300$. This thickness significantly exceeds the cell roughness. As the shear stress is constant within the boundary layer (indicated by the straight line in figure 1 of the main text), in this case the cell roughness is expected to be of minor influence on the shear stress. The consistency of our measurements, irrespective of capillary diameter and flow velocity, suggests that the roughness is of minor influence on the adhesion strength in general, i.e. even for boundary layer thickness comparable to the cell roughness. Still, a jet radius of $125 \mu \mathrm{m}$ or more is recommended for future measurements.

A detailed analysis of the flow on the single-cell scale may reveal further details of the flow-cell interaction. However, the analysis of boundary flow layer over nonflat or non-smooth surfaces is highly challenging (see for example our recent work on the flow over two-phase interfaces [42]), and a full analysis is far beyond the scope of this work.

\section{S.IV. Growth rate of the cell-free area in the dynamic regime}




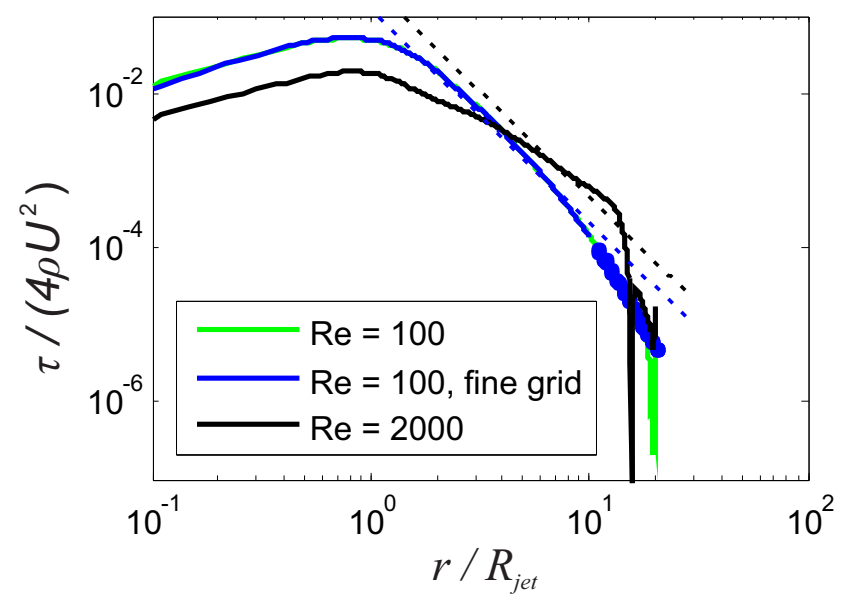

Figure S4: Shear stress calculated with Comsol solver. The dashed lines indicate Glauert's solution [16]. The peak shear stress is consistent with Ref. [18], but the far-field shear stress does not converge to Glauert's solution.

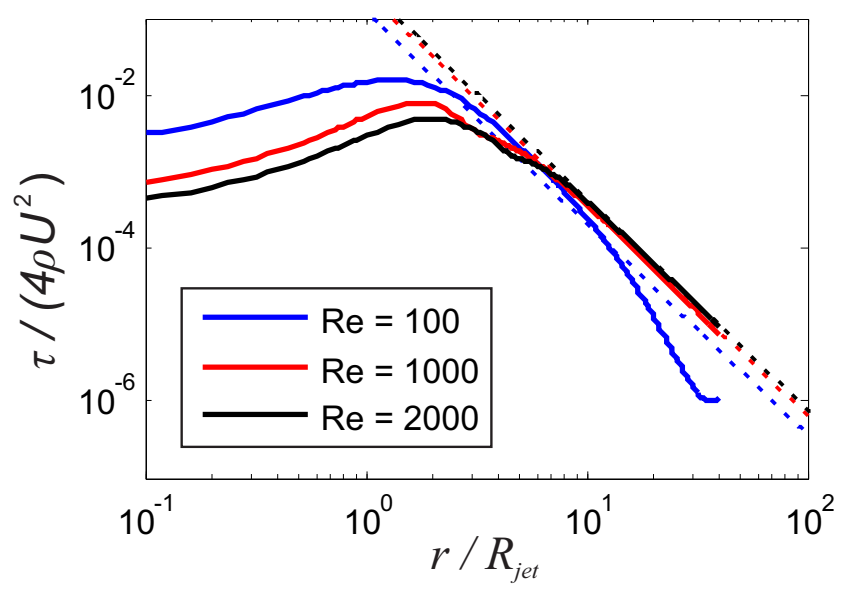

Figure S5: Shear stress calculated with Fluent solver. The dashed lines indicate Glauert's solution The far field shear stress converges to Glauert's solution but the peak shear stress is inconsistent with Ref. [18].

During the dynamic regime many cells are detached from an area close to the jet exit, due to shear stresses up to $4 \mathrm{kN} / \mathrm{m}^{2}$. To characterise the dynamic regime, the initial growth rate is defined as $\alpha=R_{\max } / t^{*}$. How does this growth rate depend on the maximal shear stress $\tau_{\max }$ ? To find out, we deduced $\tau_{\max }$ from our simulations shown 


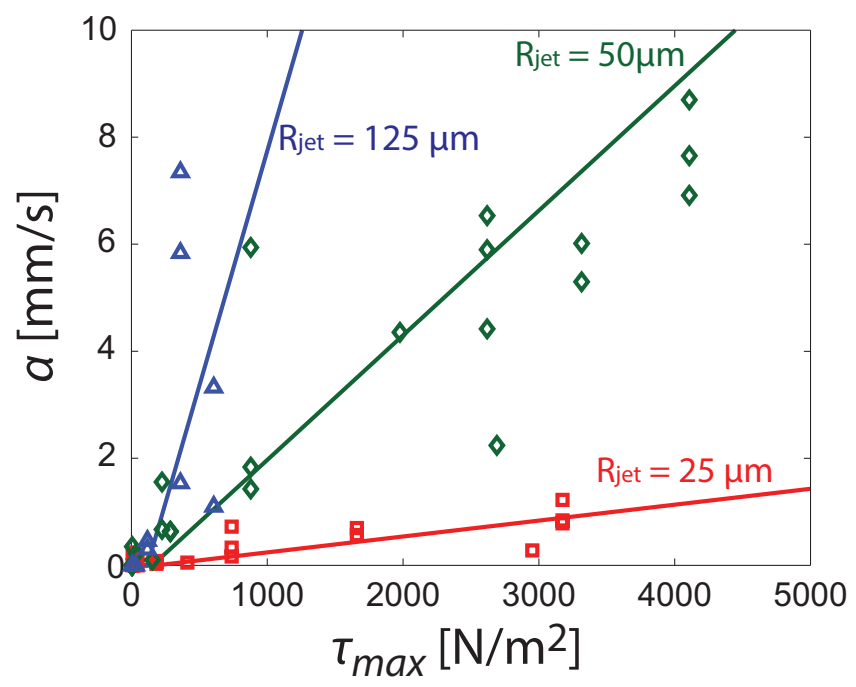

Figure S6: Initial growth rate of the cleared radius $\alpha$ versus maximal shear stress $\tau_{\text {max }}$. The lines indicate the (linear) fit $\alpha=$ const $\cdot \tau_{\max }$ for each capillary diameter. Red squares $(\square)$ correspond to $R_{\text {jet }}=25 \mu \mathrm{m}$, green diamonds $(\diamond)$ to $R_{\text {jet }}=50 \mu \mathrm{m}$, and blue triangles $(\Delta)$ to $R_{\text {jet }}=125 \mu \mathrm{m}$.

in figure S3. In figure S6, the growth rate $\alpha$ is plotted as a function of $\tau_{\max }$. We find that the growth rate is roughly proportional to the maximum shear stress applied to the cells. In figure $\mathrm{S} 6$, the linear fits $\alpha=$ const $\cdot \tau_{\max }$ are shown as straight lines, one for each cross-section of the capillary tube. Obviously, the slopes of the different lines in figure S6 are increasing for increasing capillary diameters, indicating that the employed capillary (strongly) influences the growth rate. To establish a full quantitative understanding of the diameter dependence, more measurements with different capillary diameters would be necessary. We also tried to analyze the typical detachment time scale $t^{*}$, by plotting $t^{*}$ versus either the Reynolds number or the shear rate. This resulted in a cloud of data, thus the physics governing the dynamic regime of our measurements and earlier work $[9,13]$ remains unclear.

\section{S.V. Post-jet impact cell survival}

As discussed in the main text, in some experiments, the shear stress exerted on the cells can result in poration of the cell membrane, leading to release of calcein out of the cells. Subsequent incubation of the cell monolayer with a viability marker (propidium iodide or PI) reveals possible cell death at the edge of the cleared area (figure S7). This detrimental effect of flow-induced shear stress on cells is attributed 


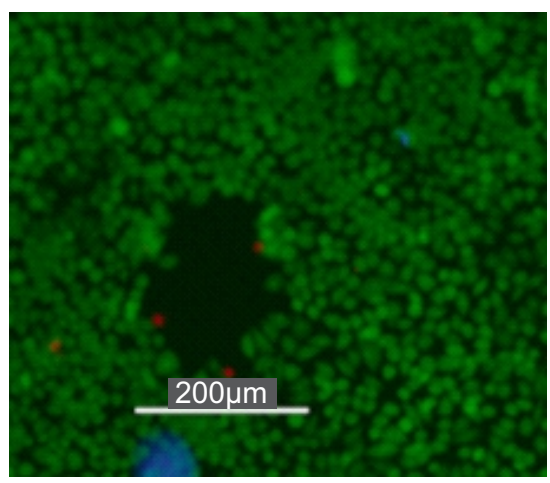

Figure S7: Live-dead assay of the cell monolayer after jetting. The cell-free area indicates where the jet impinged. At the edges of the cell-free area, a few cells have died due to excessive, shear-induced cell poration.

to the formation of (too) many pores in the cell membrane. 


\section{References}

[1] T. Dvir, B. P. Timko, D. S. Kohane, and R. Langer, Nanotechnological strategies for engineering complex tissues., Nature nanotechnology 6, 13 (2011).

[2] V. Gribova, R. Auzely-Velty, and C. Picart, Polyelectrolyte multilayer assemblies on materials surfaces: From cell adhesion to tissue engineering., Chemistry of Materials 24, 854 (2012).

[3] P. K. Thalla, H. Fadlallah, B. Liberelle, P. Lequoy, G. De Crescenzo, Y. Merhi, and S. Lerouge, Chondroitin sulfate coatings display low platelet but high endothelial cell adhesive properties favorable for vascular implants., Biomacromolecules 15, 2512 (2014).

[4] H. Ngu, Y. Feng, L. Lu, S. J. Oswald, G. D. Longmore, and F. C.-P. Yin, Effect of focal adhesion proteins on endothelial cell adhesion, motility and orientation response to cyclic strain., Annals of Biomedical Engineering 38, 208 (2010).

[5] N. Desprat, A. Richert, J. Simeon, and A. Asnacios, Creep function of a single living cell., Biophysical Journal 88, 2224 (2005).

[6] E. H. Poppele and R. M. Hozalski, Micro-cantilever method for measuring the tensile strength of biofilms and microbial flocs, Journal of Microbiological Methods 55, 607 (2003).

[7] M. Benoit, D. Gabriel, G. Gerisch, and H. E. Gaub, Discrete interactions in cell adhesion measured by single-molecule force spectroscopy., Nature Cell Biology 2, 313 (2000).

[8] R. N. Vaishnav, D. J. Patel, H. B. Atabek, M. D. Deshpande, F. Plowman, and J. Vossoughi, Determination of the local erosion atress of the canine endothelium using a jet impingement method, Journal of Biomechanical Engineering 105, 77 (1982).

[9] K. J. Bundy, L. G. Harris, B. a. Rahn, and R. G. Richards, Measurement of fibroblast and bacterial detachment from biomaterials using jet impingement., Cell Biology International 25, 289 (2001).

[10] D. C. Giliberti, K. a. Anderson, and K. C. Dee, A jet impingement investigation of osteoblastic cell adhesion., Journal of Biomedical Materials Research 62, 422 (2002).

[11] J. Kreth, E. Hagerman, K. Tam, J. Merritt, D. T. W. Wong, B. M. Wu, N. V. Myung, W. Shi, and F. Qi, Quantitative analyses of Streptococcus mutans biofilms with quartz crystal microbalance, microjet impingement and confocal microscopy., Biofilms 1, 277 (2004).

[12] A. Bouafsoun, A. Othmane, A. Kerkeni, N. Jaffrézic, and L. Ponsonnet, Evaluation of endothelial cell adherence onto collagen and fibronectin: A comparison between jet impingement and flow chamber techniques, Materials Science and Engineering: C 26, 260 (2006).

[13] S. Bayoudh, L. Ponsonnet, H. B. Ouada, a. Bakhrouf, and a. Othmane, Bacterial detachment from hydrophilic and hydrophobic surfaces using a microjet impingement, Colloids and Surfaces A: Physicochemical and Engineering Aspects 266, 160 (2005).

[14] S. Bayoudh, A. Othmane, L. Mora, and H. Ben Ouada, Assessing bacterial adhesion using DLVO and XDLVO theories and the jet impingement technique., Colloids and surfaces B: Biointerfaces 73, 1 (2009).

[15] M. Böl, A. E. Ehret, A. Bolea Albero, J. Hellriegel, and R. Krull, Recent advances in mechanical characterisation of biofilm and their significance for material modelling., Critical Reviews in Biotechnology 33, 145 (2013).

[16] M. Glauert, The wall jet, Journal of Fluid Mechanics 1, 625 (1956).

[17] C.-D. Ohl, M. Arora, R. Ikink, N. de Jong, M. Versluis, M. Delius, and D. Lohse, Sonoporation from jetting cavitation bubbles., Biophysical Journal 91, 4285 (2006).

[18] M. D. Deshpande and R. N. Vaishnav, Submerged laminar jet impingement on a plane, Journal of Fluid Mechanics 114, 213 (1982). 
[19] M. Deshpande and R. Vaishnav, Wall stress distribution due to jet impingement, Journal of Engineering Mechanics 109, 479 (1983).

[20] M. S. Gião and C. W. Keevil, Hydrodynamic shear stress to remove Listeria monocytogenes biofilms from stainless steel and polytetrafluoroethylene surfaces., Journal of Applied Microbiology 114, 256 (2013).

[21] R. Adrian and J. Westerweel, Particle Image Velocimetry (Cambridge University Press, ADDRESS, 2011).

[22] H. Schlichting and K. Gersten, Boundary-Layer Theory (Springer, New Youk, NY, USA, 2000).

[23] A. S. Goldstein and P. A. Dimilla, Application of fluid mechanic and kinetic models to characterize mammalian cell detachment in a radial-flow chamber., Biotechnology and Bioengineering 55, 616 (1997).

[24] M. M. Peel and P. A. DiMilla, Effect of cell-cell interactions on the observable strength of adhesion of sheets of cells., Annals of Biomedical Engineering 27, 236 (1999).

[25] T. G. van Kooten, J. M. Schakenraad, H. C. Van der Mei, and H. J. Busscher, Development and use of a parallel-plate flow chamber for studying cellular adhesion to solid surfaces., Journal of Biomedical Materials Research 26, 725 (1992).

[26] P. A. DiMilla, J. Stone, J. Quinn, S. M. Albelda, and D. Lauffenburger, Maximal migration of human smooth muscle cells on fibronectin and type IV collagen occurs at an intermediate attachment strength., The Journal of Cell Biology 122, 729 (1993).

[27] G. A. Truskey and J. S. Pirone, The effect of fluid shear stress upon cell adhesion to fibronectintreated surfaces., Journal of Boimedical Materials Research 24, 1333 (1990).

[28] R. Dijkink, S. Le Gac, E. Nijhuis, A. van den Berg, I. Vermes, A. Poot, and C.-D. Ohl, Controlled cavitation-cell interaction: trans-membrane transport and viability studies., Physics in Medicine and Biology 53, 375 (2008).

[29] S. L. Gac, E. Zwaan, A. van den Berg, and C.-D. Ohl, Sonoporation of suspension cells with a single cavitation bubble in a microfluidic confinement., Lab on a Chip 7, 1666 (2007).

[30] J.-C. Ochoa, C. Coufort, R. Escudié, A. Liné, and E. Paul, Influence of non-uniform distribution of shear stress on aerobic biofilms, Chemical Engineering Science 62, 3672 (2007).

[31] P. Feugier, R. Black, J. Hunt, and T. V. How, Attachment, morphology and adherence of human endothelial cells to vascular prosthesis materials under the action of shear stress., Biomaterials 26, 1457 (2005).

[32] K. V. Christ, K. B. Williamson, K. S. Masters, and K. T. Turner, Measurement of single-cell adhesion strength using a microfluidic assay., Biomedical Microdevices 12, 443 (2010).

[33] C. Cozens-Roberts, J. a. Quinn, and D. a. Lauffenberger, Receptor-mediated adhesion phenomena. Model studies with the Radical-Flow Detachment Assay., Biophysical Journal 58, 107 (1990).

[34] C.-D. Ohl and B. Wolfrum, Detachment and sonoporation of adherent HeLa-cells by shock waveinduced cavitation, Biochimica et Biophysica Acta (BBA) - General Subjects 1624, 131 (2003).

[35] R. Dijkink and C.-D. Ohl, Measurement of cavitation induced wall shear stress, Applied Physics Letters 93, 254107 (2008).

[36] K. M. Renshaw, D. E. Orr, and K. J. L. Burg, Design and evaluation of a novel flow chamber for measuring cell adhesion to absorbable polymer films., Biotechnology Progress 21, 538 (2005).

[37] V. Catto, S. Farè, G. Freddi, and M. C. Tanzi, Vascular tissue engineering: Recent advances in small diameter blood vessel regeneration, ISRN Vascular Medicine 2014, 1 (2014).

[38] S. Ravi and E. Chaikof, Biomaterials for vascular tissue engineering, Regenerative Medicine 5, 1 
(2010).

[39] N. Matsuda, T. Shimizu, M. Yamato, and T. Okano, Tissue engineering based on cell sheet technology, Advanced Materials 19, 3089 (2007).

[40] Z. Tang, Y. Akiyama, M. Yamato, and T. Okano, Comb-type grafted poly(N-isopropylacrylamide) gel modified surfaces for rapid detachment of cell sheet., Biomaterials 31, 7435 (2010).

[41] C.-H. Choi, S. Heydarkhan-Hagvall, B. M. Wu, J. C. Y. Dunn, R. E. Beygui, and C.-J. Kim, Cell growth as a sheet on three-dimensional sharp-tip nanostructures., Journal of Biomedical Materials Research. Part A 89, 804 (2009).

[42] E. Karatay, A. S. Haase, C. W. Visser, C. Sun, D. Lohse, P. A. Tsai, and R. G. H. Lammertink, Control of slippage with tunable bubble mattresses., Proceedings of the National Academy of Sciences of the United States of America 110, 8422 (2013). 


\section{9 \\ Conclusions and outlook}

Microdroplets are barely visible to the naked eye, but their impact occurs in many everyday applications. For example in spray painting, diesel engines, pressure cleaning, or when an airplane flies in a cloud and the mist droplets impact on its wings. This thesis aims to improve our understanding of these tiny droplets and apply the knowledge gained. First, the generation of individual microdroplets is pursued, since this is required to visualize their impact. Three methods have been studied: generation of a laser-induced micro-jet; deflection of a single droplet from a droplet train, and laser-induced forward transfer. Using these droplets, extensive impact experiments have been conducted of which the results will be subsequently discussed. Then, the knowledge obtained is applied to identify optimal process windows for controlled metal droplet deposition and for safe cell spraying. Finally, open questions in highspeed microdroplet impact with particular relevance for future droplet-based applications will be sketched. 


\subsection{Microdrop generation methods}

Microjet generation: High-speed microjets were generated by laser-induced vaporization of water in a micro-tube. In chapter 2 , the jet velocity has been investigated experimentally, as a function of the distance between the laser focus and the liquid meniscus at the mouth of the micro-tube, the laser energy, the liquid-glass contact angle, and the tube diameter. Varying these control parameters, controlled jets can be generated with tip velocities ranging from $5 \mathrm{~m} / \mathrm{s}$ to $850 \mathrm{~m} / \mathrm{s}$. After ejection, the tip of the jet contracts into a sphere by surface tension. This sphere detaches from the jet to form a free-flying microdroplet. The impact of such droplets was studied for velocities between $20 \mathrm{~m} / \mathrm{s}$ and $100 \mathrm{~m} / \mathrm{s}$ (chapter 3). However, the image quality is limited by motion blur, even for imaging at 1 million frames per second. This problem is solved by using a different droplet generation method, as described next. Still, to my best knowledge, laser-induced jet break-up is the only method for producing single, water(-based) drops for velocities exceeding $100 \mathrm{~m} / \mathrm{s}$.

Droplet train deflection: As described in chapter 4, a single droplet can be deflected away from a droplet train. Placing an impact surface in the path of this droplet then results in highly reproducible impact events. By carefully optimizing our setup, impact of droplets with a diameter of $50 \mu \mathrm{m}$ and velocities of $50 \mathrm{~m} / \mathrm{s}$ within a time window of $1 \mu \mathrm{s}$ was achieved, which is small and fast in comparison to previous experiments in similar setups $[1,2]$. This is sufficiently reproducible to visualize the impact using flash-illumination. In particular, synchronized side- and bottom-views show the spreading droplet at effective frame rates exceeding $10^{7}$ frames per second. Since the droplet size is sufficiently large for clear visualization and reproducible impacts can be generated, this method is very suitable to visualize the impact of small-scale droplet at high spatial and temporal resolutions.

Laser-induced forward transfer: The fastest and smallest droplets generated in this study are ejected using laser-induced forward transfer (chapter 7). In particular, a dome-shape liquid sheet can be ejected by carefully choosing the laser fluence. By surface tension, this sheet contracts into a droplet on a capillary time scale of $10^{-7} \mathrm{~s}$ (chapter 8). These droplets are less suitable for visualizing the impact dynamics, since their size is of the same order of magnitude as the wavelength of visible light.

\subsection{Drop impact fundamentals}

The similarities and differences between mm-sized droplet impact (for which great progress has been made recently) and micrometer-sized droplet impact (which is key 
for industrial applications but has hardly been addressed) are studied in detail in chapters 3 and 4. In particular, the spreading dynamics, droplet-air interaction, boundary layer development, rim development, maximum droplet spreading diameter, and splashing threshold are investigated. The main conclusions are:

1. The spreading dynamics can be divided into different phases. The initial phase, prior to the ejection of a lamella, could not be investigated due to the extremely short time scales involved. In the lamella phase, a scaling $\left.D / D_{0} \sim \sqrt{(} t / \tau\right)$ is observed, which is in quantitative agreement with mm-sized impact [3]. The final (slow-down) phase results in the maximal spreading diameter, see item 5.

2. The air bubble size entrained during microdroplet impact decreases with increasing impact velocities (for the currently studied impact velocities $V_{0}>$ $10 \mathrm{~m} / \mathrm{s}$ ). The scaling of the air bubble size collapses to results for mm-sized droplet impact in the high-Stokes number regime. This is remarkable, since incompressible air flow is a valid assumption for mm-sized droplet impact but not for micrometer-sized droplet impact $[4,5]$. The threshold Stokes number separating the high- and low Stokes number regimes is different, which is expected since this parameter is size-dependent.

3. The boundary layer thickness scales as $\bar{\delta}_{B L}(t) \sim D_{0} \operatorname{Re}^{-1 / 2}(t / \tau)^{\alpha}$, with exponent $\alpha_{B L}=0.45 \pm 0.02$. The Reynolds number dependence follows the expected scaling of $\bar{\delta}_{B L}(t) \sim D_{0} \operatorname{Re}^{-1 / 2} \sqrt{t / \tau}$, but the temporal progression is somewhat lower.

4. The rim diameter follows a scaling $D_{\text {Rim }} / D_{0} \sim \mathrm{We}^{-1 / 2}(t / \tau)^{\alpha_{\text {Rim }}}$, with exponent $\alpha_{\text {Rim }}=0.68 \pm 0.04$. To our knowledge, this scaling is observed for the first time. The temporal exponent and the remarkable robustness of this scaling, which holds for times $0.1<t / \tau<2$ and even increases for later times, remain to be explained.

5. The maximal droplet spreading diameter is robustly captured by balancing the inertia of the droplet to viscous dissipation in the boundary layer [6].

6. Despite our high impact velocities up to $V_{0}=50 \mathrm{~m} / \mathrm{s}$, no splash is observed here. Only models including the surrounding air $[7,8]$ correctly predict this outcome. These models are based on scaling arguments, and do not contain details of the physics of splashing. Alternative models capture the physics of splashing in more detail $[9,10]$, but since the continuum assumption is violated for the thin air film between the droplet and the substrate these models are not applicable. 
As far as could be observed, droplet impact is scale-invariant, i.e. mm-sized droplet experiments have a predictive value as long as the same Reynolds, Weber, and Stokes number are used. Therefore, the vast progress in understanding mm-sized droplet impact can be applied to optimize a wide range of applications in which fast, $\mu \mathrm{m}$ sized droplet impact on a dry surface is a key process step.

\subsection{Microdroplets as a technology enabler}

Laser-induced forward transfer: For the first time, laser-induced forward transfer was applied for the fabrication of three-dimensional metal microstructures (chapter 8). By repeating the ejection of copper micro-droplets at the same location, their deposition occurs on the same position and pillars are manufactured. These pillars have low porosity, are electrically conductive, and mechanically stable. The LIFT concept was extended to deposit lines, fill through-silicon vias, and make $2 \mathrm{~mm}$ long pillars with a diameter of $5 \mu \mathrm{m}$. As stacked deposition has been an unfulfilled promise since the conception of LIFT in 1986 [11], these results demonstrate a major step in its applicability.

To construct these pillars, controlled sizes and impact velocity of the impacting droplets are prerequisites. Therefore, in-depth knowledge of the droplet's ejection regime and ejection velocity, as described in chapter 7, has been crucial. In particular, in a novel regime called "cap"-ejection, the ejection of a hemispherical piece of the film is observed which contracts into a droplet. Such droplets are used to fabricate the aforementioned pillars. However, if the ejection velocity is too low, surface tension will prevent detachment of the cap from the donor. This occurs for a Weber number We $\lesssim 1$, which corresponds to a minimal ejection velocity of $\approx 20 \mathrm{~m} / \mathrm{s}$. The fluence can also be too high, resulting in ejection of many droplets in regimes called "jet-ejection" and "spray ejection". As the driving mechanisms and transitions between these regimes were elucidated, the fluence and the properties of the donor film can now be tuned to achieve ejection in a specific regime. In view of the well-defined droplets ejected in the cap ejection regime, this regime seems to be promising for future micro-manufacturing applications.

Cell spray deposition: In treatment of burns and biofabrication, cells deposited by spraying (also known as air-brush) is currently introduced. To optimize the cell survival in these applications, the influence of the spray control parameters (liquid flow rate, liquid viscosity, and gas pressure) and the substrate stiffness on cell survival were quantitatively investigated. A two-step approach was followed: First, the influence of the spray conditions on the droplet size and velocity was experimentally 
quantified, using flash photography. This revealed that especially increasing the air pressure results in faster impact velocities of the droplets. Second, an analytical cell-survival model was presented to describe (1) the cell survival probability as a function of cell membrane elongation, (2) the membrane elongation as a function of the cell-containing droplet impact parameters, and (3) the influence of the impact substrate properties on membrane elongation. This model predicts improved cell survival for larger surrounding droplets, slower impact, lower droplet viscosity, and soft-surface impact. These predictions are successfully validated with cell viability measurements, which demonstrates the importance of controlled droplet impact in a range of biofabrication technologies. The model and experimental setup allow for optimization of any droplet-based cell deposition technology. In the particular case of (clinical) cell spraying, post-spray cell viability is improved by decreasing the air pressure applied to the spray nozzle and increasing the nozzle-substrate distance.

\subsection{Outlook}

In the context of this thesis, some aspects of droplet impact seem to be fruitful to investigate in the future. First, violent shock waves can form in high-velocity impact onto liquids or solids [12], but their incidence has been insufficiently addressed to answer practical questions. For example, it is not known whether the water hammer effect (also known as "hydraulic shock") plays an important role in the cleaning of semiconductor wafers or hot-rolled steel (descaling). The role of ambient air and shock waves in the liquid may be closely related, since the influence of air is known to dampen the droplet's impact. Therefore, shock formation might be enhanced by decreasing the pressure of the surrounding gas, potentially improving cleaning applications. Although recently the role of air has been described for low impact velocities $[4,5]$, theory, simulations, and experiments for fast micro-droplet impact are still lacking.

Second, the shear rates and shear stresses related to droplet impact deserve attention. For example, cell viability in bioprinting seems to strongly decrease beyond a certain critical shear stress. Also, the shear stress exerted to the impact substrate in cleaning applications is not adequately known. Since measuring and calculating the shear during impact are within reach of current experimental (micro-PIV) and numerical methods, this is a very promising topic.

Finally, for non-wetting impact at very high velocity, holes can form in the lamella ejected during impact and eventually cause break-up of the lamella into small droplets [13]. This phenomenon affects applications ranging from thermal spraying to laserinduced droplet shaping. However, the origin of these holes is neither understood 
sufficiently to predict their formation, nor visualized as a function of relevant control parameters. Therefore, the incidence of this regime is essentially unknown. Using flash photography [13] and molecular-dynamics situations [14] to visualize the incidence of these holes might allow to explain their formation mechanism.

\section{References}

[1] N. R. Lindblad and J. M. Schneider, Production of uniform-sized liquid droplets, Journal of Scientific Instruments 42, 635 (1965).

[2] H.-Y. Kim, S.-Y. Park, and K. Min, Imaging the high-speed impact of microdrop on solid surface, Review of Scientific Instruments 74, 4930 (2003).

[3] A. Mongruel, V. Daru, F. Feuillebois, and S. Tabakova, Early post-impact time dynamics of viscous drops onto a solid dry surface, Physics of Fluids 21, 032101 (2009).

[4] P. D. Hicks and R. Purvis, Air cushioning in droplet impacts with liquid layers and other droplets, Physics of Fluids 23, 062104 (2011).

[5] P. D. Hicks and R. Purvis, Liquidsolid impacts with compressible gas cushioning, Journal of Fluid Mechanics 735, 120 (2013).

[6] I. V. Roisman, Inertia dominated drop collisions. II. An analytical solution of the NavierStokes equations for a spreading viscous film, Physics of Fluids 21, 052104 (2009).

[7] L. Xu, W. Zhang, and S. Nagel, Drop splashing on a dry smooth surface, Physical Review Letters 94, 184505 (2005).

[8] C. S. Stevens, Scaling of the splash threshold for low-viscosity fluids, EPL (Europhysics Letters) 106, 24001 (2014).

[9] S. Mandre and M. Brenner, The mechanism of a splash on a dry solid surface, Journal of Fluid Mechanics 690, 148 (2012).

[10] G. Riboux and J. Gordillo, The critical impact speed for the splash of a drop, arXiv preprint arXiv:1401.6943 1 (2014).

[11] J. Bohandy, B. Kim, and F. Adrian, Metal deposition from a supported metal film using an excimer laser, Journal of Applied Physics 60, 1538 (1986).

[12] J. Field, J. Dear, and J. Ogren, The effects of target compliance on liquid drop impact, Journal of Applied Physics 65, 533 (1989).

[13] A. McDonald, M. Lamontagne, C. Moreau, and S. Chandra, Impact of plasma-sprayed metal particles on hot and cold glass surfaces, Thin Solid Films 514, 212 (2006).

[14] R. Zhang, S. Farokhirad, T. Lee, and J. Koplik, Multiscale liquid drop impact on wettable and textured surfaces, Physics of Fluids 26, 082003 (2014). 


\section{Summary}

The impact of mm-sized droplets at around $1 \mathrm{~m} / \mathrm{s}$ can be observed every day, for example in rain, in the sink or shower, or during sailing. However, the impact of much faster, micrometer-sized droplets is at least as omnipresent. Such impacts cannot be seen with the naked eye, but occur in applications including spray painting, inkjet printing, diesel engines, or pressure cleaning. In this thesis, the dynamics of the $\mathrm{mm}$-sized droplets (for which many experiments have been performed) are compared to $\mu \mathrm{m}$-sized droplets (which have received scarce attention), and the knowledge is implemented to enable and improve novel applications.

First, the generation and controlled impact of individual microdroplets is pursued. Three methods have been studied and will now be concisely described: generation of a laser-induced micro-jet; deflection of a single droplet from a droplet train, and laser-induced forward transfer.

(i) High-speed microjets were generated by laser-induced vaporization of water in a micro-tube (chapter 2). The jet velocity has been investigated experimentally, as a function of the distance between the laser focus and the liquid meniscus at the mouth of the micro-tube, the laser energy, the liquid-glass contact angle, and the tube diameter. Varying these control parameters, controlled jets can be generated with tip velocities ranging from $5 \mathrm{~m} / \mathrm{s}$ to $850 \mathrm{~m} / \mathrm{s}$. After ejection, the tip of the jet contracts into a sphere by surface tension. This sphere detaches from the jet to form a freeflying microdroplet. The impact of such droplets was studied for velocities between $20 \mathrm{~m} / \mathrm{s}$ and $100 \mathrm{~m} / \mathrm{s}$ (chapter 3).

(ii) Alternatively, a single droplet can be deflected away from a droplet train (chapter 4). Placing an impact surface in the path of this droplet then results in highly reproducible impact events. By carefully optimizing our setup, impact of droplets with a diameter of $50 \mu \mathrm{m}$ and velocities of $50 \mathrm{~m} / \mathrm{s}$ within a time window of $1 \mu \mathrm{s}$ was achieved, which is small and fast in comparison to previous experiments in similar setups. This time window is sufficiently short to visualize the impact using flashillumination. In particular, synchronized side- and bottom-views show the spreading droplet at effective frame rates exceeding $10^{7}$ frames per second. Since the droplet size is sufficiently large for clear visualization and reproducible impacts can be gen- 
erated, this method is very suitable to visualize the impact of small-scale droplet at high spatial and temporal resolutions.

(iii) The fastest and smallest droplets generated in this study are ejected using laserinduced forward transfer (chapter 7). In particular, a dome-shape liquid sheet can be ejected by carefully choosing the laser fluence. By surface tension, this sheet contracts into a droplet on a capillary time scale of $10^{-7} \mathrm{~s}$ (chapter 8 ). These droplets are less suitable for visualizing the impact dynamics, since their size is of the same order of magnitude as the wavelength of visible light and they solidify upon impact.

Microdroplet impact is equivalent to mm-sized droplet impact. This conclusion is based on extended droplet impact experiments (chapters 3 and 4), in which the spreading dynamics, droplet-air interaction, boundary layer development, rim development, maximum droplet spreading diameter, and splashing threshold are comprehensively discussed. The implication is that mm-sized droplet impact experiments can be tailored to make predictions for $\mu \mathrm{m}$-sized droplets impacting at higher velocities, as long as the Weber, Reynolds, and Stokes numbers are maintained equal. Therefore, the vast progress in understanding mm-sized droplet impact can be applied to optimize a wide range of applications in which fast, $\mu \mathrm{m}$-sized droplet impact on a dry surface is a key process step.

Finally, the knowledge obtained is applied for the development of novel technologies. For the first time, laser-induced forward transfer was applied for the fabrication of three-dimensional metal microstructures (chapter 8). In laser-induced forward transfer, a pulsed laser is focused on a thin metal film, which results in the ejection of small copper droplets. By repeating the ejection of copper micro-droplets at the same location, their deposition occurs on the same position and pillars are manufactured. These pillars have low porosity, are electrically conductive, and mechanically stable. The LIFT concept was extended to deposit lines, fill through-silicon vias, and make $2 \mathrm{~mm}$ long pillars with a diameter of $5 \mu \mathrm{m}$. As stacked deposition has been an unfulfilled promise since the conception of LIFT, these results demonstrate a major step in its applicability.

Biofabrication is another important and novel application of microdroplets. Here, cell-containing droplets are deposited in a controlled manner, with the aim to grow functional tissues or even organs from the deposited droplets. However, the cells do not always survive the impact event, which is a severe problem in several mainstream biofabrication technologies. Therefore, we model the cell deformation during impact, by considering a single cell and assuming that it deforms equivalent to a liquid droplet. The cell's deformation is connected to the expected cell viability, and predicted using an existing cell viability model. Next, we include the influence of the droplet which surrounds the cell in our models (in particular, the surrounding 
droplet size and viscosity are included), as well as the stiffness of the surface. This model predicts improved cell survival for larger surrounding droplets, slower impact, lower droplet viscosity, and soft-surface impact. These predictions are successfully validated with cell viability measurements, which demonstrates the importance of controlled droplet impact in a range of biofabrication technologies. 


\section{Samenvatting}

Tijdens een regenbui, in de douche, of op een zeilboot wordt de inslag van druppels op een oppervlak aan den lijve ondervonden. Het alledaagse voorkomen van druppelinslag en de eenvoud van een bolvormige druppel die inslaat op een vlak oppervlak doet vermoeden dat de inslagdynamica eenvoudig is, maar schijn bedriegt. Tijdens de inslag, die slechts enkele milliseconden duurt, spreidt de druppel uit tot een bepaalde maximale diameter. Vervolgens kan de druppel samentrekken tot een bolvorm (op hydrofobe oppervlakken zoals was of bepaalde plantenbladeren), steeds kleiner worden tijdens zijn verdamping, of zijn maximale diameter behouden totdat hij verdampt is. Daarnaast kunnen druppels uiteenspatten in meerdere druppels. Dit kan bijvoorbeeld zichtbaar worden gemaakt door koffiedruppels te laten vallen vanaf enkele meters hoog, waarna een patroon van kleine koffiedruppels op de vloer zichtbaar wordt.

De laatste jaren is er veel onderzoek is gedaan naar dergelijke huis- tuin- en keu-kendruppels. Door middel van hogesnelheidsfotografie zijn de spreidingsdynamica en de maximale diameter tijdens inslag in beeld gebracht. Daarnaast is ontdekt dat, tijdens een aanzienlijk deel van de inslag, de druppel het inslag-oppervlak niet "aanraakt", maar de druppel gescheiden blijft van het oppervlak door een dunne luchtlaag met een dikte van enkele nanometers tot enkele micrometers. Tenslotte is de uiteenspatting tijdens inslag uitgebreid onderzocht. Hoewel de details van dit fenomeen nog onbekend zijn, blijkt dat ook hier de invloed van de omringende lucht essentieel is om te beschrijven wanneer fragmentatie zal optreden. Concluderend kan gesteld worden dat belangrijke details nog verdere aandacht verdienen, maar dat uitgebreid onderzoek desondanks heeft geleid tot een goed overzicht van de inslagdynamica van druppels met een grootte van enkele millimeters en een snelheid van enkele meters per seconde.

In dit proefschrift wordt de inslag-dynamica van kleine en snelle druppels vergeleken met die van veel grotere druppels waar reeds uitgebreid onderzoek naar is verricht. Deze druppels, met een diameter van 10 tot 100 micrometer en een snelheid van 10 tot $100 \mathrm{~m} / \mathrm{s}$, worden bijvoorbeeld gebruikt in printers, dieselmotoren, hogedrukspuiten en vele andere toepassingen. Ondanks deze relevantie is de inslagdynamica 
van deze druppels nauwelijks gevisualiseerd, omdat een inslag moeilijk te realiseren is. Daarnaast vindt de inslag plaats in een tijdsbestek van slechts enkele microseconden, en is dus ook moeilijk te visualiseren. Dit proefschrift heeft daarom een drietal doelen: het maken, visualiseren, en toepassen van microdruppels.

De inslag van individuele druppels is bereikt door middel van drie verschillende methoden. Ten eerste is gebruikt gemaakt van een (potentieel supersonische) micro-jet (hoofdstuk 2 en 3). Hierin wordt een laser gefocust in een vloeistof die zich bevindt in buisje met een open uiteinde. De laser veroorzaakt een kookbel en een drukgolf, en heeft tot gevolg dat een extreem snelle (tot $\sim 1000 \mathrm{~m} / \mathrm{s}$ ), microscopisch kleine vloeistofstraal wordt weggespoten vanaf het open vloeistofoppervlak. Deze straal breekt vervolgens op in losse microdruppels, die inslaan op een daartoe geplaatst oppervlak. Vervolgens is de inslag bekeken door middel van hogesnelheidsfotografie. Hoewel de druppels duidelijk zichtbaar zijn, is achteraf gebleken dat fotografie met 1 miljoen beelden per seconde onvoldoende detail (zowel in de tijd als in de plaats) biedt om de inslag nauwkeurig vast te leggen.

Daarom is ten tweede een methode uitgewerkt om extreem reproduceerbare druppelinslag te bereiken (hoofdstuk 4). Het voordeel is dat nu een 6 nanoseconde korte lichtflits gebruikt kan worden om de druppel vast te leggen op een specifiek moment van de inslag. Door de vertraging tussen de druppelinslag en de lichtflits te variëren, kan op deze manier de gehele inslagdynamica in beeld worden gebracht. De basis van de deze methode is om een "trein" van druppels met gelijke grootte en snelheid te maken (dit kan vergeleken worden met een tennisballenkanon waarmee zo frequent tennisballen worden weggeschoten dat er een "trein" van tennisballen door de lucht vliegt). Dit is relatief eenvoudig, maar heeft als probleem dat inslag op een droog oppervlak vrijwel onmogelijk is omdat alle eerdere druppels het oppervlak benatten. Daarom wordt er één druppel uit de trein elektrisch geladen, en vervolgens wordt de gehele trein door een elektrisch veld geleid. Hierdoor wordt uitsluitend de geladen druppel beïnvloed, die vervolgens een traject volgt dat iets afbuigt van de trein. Vervolgens wordt er een dunne scheidingswand aangebracht tussen het traject van de geladen druppel en de trein, zodat alleen de geladen druppel overblijft, die vervolgens inslaat op het gewenste oppervlak. Op deze manier ontstaat een zeer reproduceerbare inslag, die succesvol is vastgelegd met een gecombineerd en gesynchroniseerd zij- en bodemaanzicht.

Deze druppels zijn in detail vergeleken met eerdere resultaten voor grotere, langzamere druppels. De spreidingsdynamica, maximale spreidingsdiameter, de ingevangen luchtbel, het al of niet plaatsvinden van de uiteenspatting van de druppel zijn gemeten. Deze parameters kunnen berekend worden met recente modellen die uitsluitend gevalideerd zijn voor grotere druppels, dus de druppel-dynamica is schaal- 
baar voor zover kon worden beoordeeld. Dit betekent dat de hiervoor genoemde toepassingen geoptimaliseerd kunnen worden met kennis die wordt vergaard voor veel grotere druppels, die wèl eenvoudig in beeld gebracht kunnen worden.

Ten derde is er een methode ontwikkeld om reproduceerbare metaaldruppels te maken (hoofdstuk 5 en 6). Deze druppels zijn zo klein (met een diameter tot 3 micrometer) en snel (20 tot $200 \mathrm{~m} / \mathrm{s}$ ) dat ze zelfs met flitsfotografie nauwelijks gedetailleerd vastgelegd kunnen worden. Echter, met deze druppels zijn voor de eerste keer 3D structuren geprint van pure metalen met een hoog smeltpunt (koper en goud), in de vorm van pilaren van enkele micrometers in diameter en (tot) enkele millimeters hoog. Deze extreem dunne pilaren hebben een lage porositeit, zijn mechanisch stabiel en electrisch geleidend, en zijn daarom uitstekend geschikt om verticale electronische circuits te printen. Daarnaast kan gedacht worden aan de depositie van micro-spijkerbedden zoals gebuikt voor hydrofobe oppervlakken, of micro-antennes voor het verder verkleinen van bepaalde radio-toepassingen.

Daarnaast is de interactie tussen levende cellen en microdruppels onderzocht. Dit betreft cellen die zich binnen een microdruppel bevinden die inslaat op een oppervlak. Dergelijke inslag ligt aan de basis van diverse technieken in biofabricatie, een vakgebied dat tot doel heeft om gezond (levend) weefsel te ontwikkelen ter vervanging van beschadigd of niet-functioneel weefsel in het lichaam. Hierbij wordt een suspensie bestaande uit vloeistof en cellen opgedeeld in microdruppels die vervolgens worden weggeschoten en terecht komen op het te printen oppervlak.

Het is gebleken dat met name cel-deformatie tijdens de inslag een belangrijke oorzaak is van schade aan de cellen (hoofdstuk 7). De overlevingskans van de cellen is gemodelleerd en gemeten, en het model is in goede overeenstemming met de gemeten resultaten. Onder andere is gebleken dat de overlevingskans van cellen toeneemt voor grotere omringende druppels, lagere inslagsnelheid, minder visceuze vloeistoffen en zachtere oppervlakken. Voor optimale overleving van de cellen in de cel-spray methode wordt aanbevolen om een lage luchtdruk toe te passen, waardoor de druppelgrootte toeneemt en de inslagsnelheid afneemt.

Tenslotte is de inslag van een "microjet" (waterstraaltje met een diameter tot 500 micrometer) op een oppervlak dat bedekt is met een monolaag cellen onderzocht (hoofdstuk 8). Hierbij is de microjet loodrecht op het oppervlak gericht. De stroming van de microjet heeft tot gevolg dat de cellen weggespoeld worden worden in een cirkelvormig cel-vrij gebied dat groeit in de tijd. Na enige tijd ontstaat echter een stabiele situatie, waarin de diameter van het cel-vrije gebied niet langer groeit. Een meting van deze diameter kan vervolgens gebruikt worden om de adhesie van de cellen aan het oppervlak te kwantificeren.

Hoewel deze methode welbekend is, was hij onvoldoende gekarakteriseerd voor 
betrouwbare toepassing. Daarom is in hoofdstuk 8 de stroming van de microjet zowel gemeten als gemodelleerd, de tijdsduur waarna de stabiele situatie optreedt onderzocht, en het onthechtingspatroon van de cellen in real-time in beeld gebracht. Hieruit blijkt dat de stabiele situatie binnen enkele seconden optreedt, en vervolgens de adhesie van de cellen aan het oppervlak berekend kan worden met eenvoudige modellen, met als vereiste dat de stroming van de microjet laminair is. Daarnaast zijn verschillende regimes waargenomen voor het loslaten van de cellen. Voor een monolaag met een celdichtheid lager dan $(1.8 \pm 0.2) \cdot 10^{9}$ cellen $/ \mathrm{m}^{2}$ laten de cellen individueel los van het oppervlak, terwijl intacte stukken van de monolaag loslaten voor hogere celdichtheden. Dergelijke intacte celfilms, die bijvoorbeeld worden toegepast in biofabricatie, kunnen op deze manier in zeer korte tijd worden gescheiden van het oppervlak. 


\section{Acknowledgements}

"Als je een plant wilt laten groeien moet je hem niet aan zijn

stengel trekken, maar water geven." *

- Pieter Cornelis Visser (1954-2005), My father

After finishing my master in applied physics, several job options were on the table. Two of the offers were PhD assignments offered by Detlef Lohse, chair of the very same Physics of Fluids (PoF) group in which I am currently completing my dissertation. I still remember reading them on the train: the first was on rotating Rayleigh Bénard convection, and the other one on the understanding and optimization of ink-jet printing in collaboration with Océ. However, in my view at the time, these assignments were highly fundamental. Since I preferred (and prefer) to be able to explain the relevance of my job to my grandmother, I politely declined both assignments (which meanwhile have been carried out by others in truly excellent ways, and did have a clear relevance which I meanwhile came to appreciate), and, after a short excursion into economics, I found my first real job at Tata Steel.

This story is however only complete with Detlef's remark on my departure, which clearly indicated that "Some day, people like you return to academia to do 'real science" ". Detlef, among other things, thank you for these words. You did not criticize my decision but left your door open for a PhD project, for which this thesis marks the completion. During my four years in industry, I formed a more complete judgement of both academic and industrial research, which made me realize that there was still much to learn in academia without losing societal relevance. We started discussing $\mathrm{PhD}$ options, which eventually resulted in my return to the group on your FOM-grant. During my PhD project, your critical judgement and honesty have taught me scientific rigor and quality, and you involved me in collaborations which have resulted in great publications and more to come. Detlef, thank you for supporting my past, present, and future research in many ways, and nurturing the atmosphere in the PoF group in which I enjoyed practically every day of my stay.

${ }^{*}$ To make a plant grow you should not pull its stem, but water it. 
Chao, we got to know each other long ago, during a great number of enjoyable lunches and dinners at the Chinese University of Hong Kong (chi-fan!!). You were working hard on your $\mathrm{PhD}$, I was an internship student, and I believe neither of us could foresee that one day you would be my PhD supervisor. Chao, you are an exemplary supervisor, for which I am very grateful and consider myself very lucky. Your supervision is daily when needed and loose if possible. You provide clear guidelines but let me make my own mistakes, which I both truly appreciate. Our talks spanned from the smallest details of droplet impact to major scientific booby-traps, and from family issues to future career planning. Last but not least, your special sensor for incomplete or flawed story-lines has consistently helped me to gain new insights and forced me to overhaul (and significantly improve) several article drafts. Chao, thank you for your great help. I look forward to our collaboration in both the near and the far future.

Several chapters of this thesis are the results of collaborations with people from other groups, both at the University of Twente (UT) and abroad. The cornerstone chapter 4 of this thesis is the result of an extensive collaboration with the University of Göttingen and LAM Research. Philipp Frommhold, it was a great pleasure to work with such an excellent experimentalist; let's see what the future will bring for us! Robert Mettin, Alexander Lippert, and Harald Okorn-Schmidt, many thanks for your support. I hope to maintain our fruitful collaboration!

The work on laser-induced forward transfer (LIFT) was done in close collaboration with the Applied Laser Technology group at the UT. Ralph Pohl, many thanks for a long-time, productive, and enjoyable collaboration which has resulted in a deep and still developing understanding of the fascinating physics involved in LIFT. Bert Huis in 't Veld and Gert-Willem Römer, your continued and strong support has been invaluable, and will hopefully keep nurturing our research in the future.

The "biological" part of the thesis was initiated by Marise Gielen's MSc project, and would not have been possible without Séverine Le Gac and Zhenxia Hao from the BIOS group. Thank your for your continuous and constructive support until the final and sometimes difficult stages of writing. I am very happy to finally indicate chapter 8 as "accepted"!

Subsequently, the cell-related work shifted to cell survival in cell spraying. In particular, the influence of the spraying parameters was believed to sometimes result in significant cell death. Jan Hendriks, a master student at the time, and me were kindly asked to experimentally investigate the spray process, which eventually resulted in the insights presented in chapter 7 of this thesis. Jan, Sieger Henke, and Marcel Karperien, thank you for many challenging and fruitful discussion and excellent time spent in the lab (or running MATLAB). We will continue our collaboration, 
and hopefully accommodate many of the follow-up ideas we gained over the past two years.

Last but not least, I enjoyed the collaboration with the Soft Matter, Fluidics and Interfaces group on the slip length over bubble mattresses. Elif Karatay and Sander Haase, many thanks for doing such excellent (sometimes explosive) lab work and numerical modeling. Elif, Sander, Amy Tsai, Álvaro Marín, and Rob Lammertink, this project was my first collaboration and allowed me to learn in many ways. I thank you for your kind support and patience in all stages involved.

During my $\mathrm{PhD}$, I had the privilege of meeting many interesting people around the globe. Many thanks to the participants of the summer schools in Les Houches and Kogerup, the APS-DFD conferences, and the Biofabrication workshop, for making them scientifically challenging and socially enjoyable. Closer to home, I joined the program committee of the Studium Generale at the UT. Peter Timmerman, Anneke Heukels, Hiska Bakker, and fellow committee members, thank you for the inspiring brainstorms.

I also had the great pleasure to work with several excellent master, bachelor, and internship students. Sander, Marise, Jan, Nikolai Oudalov, Martin Klein Schaarsberg, Sofia Oliveira, Thomas Bredenoord, Boris Boom and Hille Groendijk, Sebastian Sterl, Toshiaki Fukada, and Pei-Hsun Tsai, I have tried to practice the quote above this chapter in your supervision, with the intention to make you stronger. For each of you, I wish a future that best suits you as a person. Also the collaboration with fellow PhD students at the UT was great. Alexander Klein, Sander Wildeman, Yali Zhang, Pascal Sleutel, thank you - and see you soon.

My daily work was primarily carried out in office ME250. Álvaro, Koen Winkels, Laura Stricker, Henri Lhuissier, Rianne de Jong, Vamsi Spandan, and Loreto Oyarte, thanks for excellent scientific discussions, making our office a welcome place, and ensuring good company at virtually any party in Enschede and surroundings. The work could only be carried out due to the assistance of Joanita Leferink, Gert-Wim Bruggert, Martin Bos, Bas Benschop, even though my requests usually came on short notice. Jacco Snoeijer, Tess Homan, and Peter van Dijk, thanks for teaching together, I hope the students learned a lot and I did for sure! Guillaume Lajoinie, Sander Huisman, Tim Segers, Mark-Jan van der Meulen, Minori Shirota, Antonin Eddi, Michiel van Limbeek, Hanneke Gelderblom, Vivek Prakash, Varghese Mathai, Oscar Enríquez, Theo Driessen, Wilco Bouwhuis, Tuan Tran, Marie-Jean Thoraval, Stefan Karpitschka, Arjan van der Bos, Bram Verhaagen, Yoshiyuki Tagawa, Erik Dietrich, Federico Hernández Sánchez, Rodolfo Ostilla Mónico, Elena Castro-Hernández, Enrique Sandoval Nava, Huanshu Tan, and many others (the list paradox: Longer lists are less complete), thank you for scientific discussions, teaching me how to use equip- 
ment, Friday-afternoon experiments, various borrels, assistance with editing this thesis, borrowing and lending of all kinds of stuff, or making available lab space. These are not only required for scientific progress, but also keep life enjoyable for all involved in the PoF-lab-ecosystem.

The years in Tata Steel have shaped me, and had a great influence on my $\mathrm{PhD}$ project. Jaap van der Lugt, Jochem Groot, Jan de Roo, Henk Bolt, Wanda "Caro" Melfo, David Matthews, and Harry van Steden, thank you for giving me unparalleled opportunities to develop and your open doors when in came to future career steps. Marc de Boer, Koert van Eijk, Dirk-Jan Wentink, Renske Nelissen, Peter Gelten, Maaike van der Laan, Jan Ponsioen, Camile Hol, and many others, the good times have not been forgotten!

Over the past years, I have been lucky to share my life with a large group of great friends. Wouter and Sirée, meeting (/hiking/climbing/gaming) with you is always a joy! Wim, Katharine, Maarten, Marjon, Joanne, Bernd, Rudi, Hannah, Hesther, Johan, Marten, Christiana, Cees, Harmen, Bart, Sander, Johanna, and Maarten, we met many years ago and are still going strong. It is always fantastic to see you, and I hope we maintain our friendship for many, many years to come. Pieter, Marjanne, and Corianne, we got to know each other in Enschede, but you became friends in Amsterdam. Thanks for many nice evenings. Manus, Jaap, Dorine, Renske, Thomas, Menno, Anne, Joris, Sjaak, Frits, Roger, and many others, thank you for truly enjoyable, fast, and relaxed sailing trips all over Europe. Gijs, topman, and Esther, we had a great time in Amsterdam and I enjoyed our sailing. To be continued!

Het is een geluk om deel uit te maken van een grote en groeiende familie. Mam, je bent een fantastische moeder die altijd klaar staat met echte steun. Anne en Rick, jullie zijn een geweldige zus en broer, en ik hoop op veel moois voor jullie en onze toekomst. Lieve Oma, ik heb genoten van alle activiteiten met en voor de hele familie, en hoop dat we dit nog heel lang kunnen blijven doen. Tante Marie, heel veel dank voor alle steun en betrokkenheid door de jaren heen, en in het bijzonder tijdens de eerste jaren van mijn studie.

Dear Gül, words are inadequate to describe my gratefulness for your love, support, encouragement, patience, and advice, both with regards to the thesis and everything beyond it. Mijn lief, thank you so much.

And now, let's celebrate!

Enschede, December 2014 


\section{About the author}

Claas Willem Visser was born in Haarlem in 1981. In the first five years of his life he lived in Haarlem, Lelystad, Maastricht, and Haren (Groningen). Here he grew up while launching water missiles and being actively involved in youth society 't Vooronder, amongst other activities, until moving to Enschede for studying at the University of Twente (UT) in 1999. The first years of studying mainly concerned introductory courses on the experimental, theoretical, and mathematical aspects of applied physics. Here, fluid mechanics were first introduced to him, revealing an interesting combination of "hands-on" experiments and complex underlying phenomena that can sometimes be described in simple equations. This combination raised his interest and materialized in the following phases of studying. Besides these scholar activities, Claas Willem became a member and later chairman of student association CSV Alpha.

In the later phases of studying, Claas Willem joined the Physics of Fluids group chaired by Prof. Detlef Lohse. From this base, he went for his internship to the Chinese University of Hong Kong where he studied the roles of plumes in a turbulent flow supervised by Prof. Ke-Qing Xia and Dr. Heng Dong Xi, and at the same time completed his minor program "International Management". Subsequently, under the supervision of Detlef Lohse and Hanneke Bluemink, he finished his master thesis Dynamics of particles in a rotating cylinder. During these years, sailing became a major side-activity, involving sea-going cruising trips and Laser-II racing on world and European championships.

After completing his master, Claas Willem moved to Amsterdam and joined the Rolling Metal Strip department at Tata Steel. Here, he learned to practice his academic knowledge to solve industrial problems, and later became a research project leader. This project leadership grew into several projects, including EU-funded (RFCS) research projects in which collaborations with the Center for Research on Metals (Center for Research of Metals, Ghent), Brno University, and several hot strip mills in Tata Steel were started or extended. He also became secretary of the Tata global working group on rolls in hot strip mill rolling, where he contributed to sharing best practices between work roll users and developers in Tata Steel's hot strip mills. 
The fruitful years at Tata Steel were followed by Claas Willem's PhD project, for which he returned to the Physics of Fluids group. Here, he studied the fundamentals and applications of small-scale liquid droplets and jets, building on his prior knowledge and experience. In particular, he demonstrated the importance of droplet impact in cell delivery in biofabrication and pure-metal printing of copper and gold pillars. During his PhD he set up or extended collaborations with groups within the UT and abroad, which have resulted in several chapters of this thesis, and he co-organized the FLOW14 conference. He is a member of the program committee of the Studium Generale of the UT, in which he contributes to the annual program. And, as always, he still enjoys sailing, hiking, and spending time with friends. 


\section{Scientific output}

\section{Publications}

\section{Peer-reviewed journal articles}

1. Visser, C.W., Gielen*, M.V., Hao, Z., Le Gac, S., Sun, C., \& Lohse, D. “Quantifying cell adhesion through impingement of a controlled microjet.” Biophysical Journal (accepted for publication).

2. Karatay, E., Haase, A.S., Visser, C.W., Sun, C., Lohse, D., Tsai, P.A., \& Lammertink, R.G.H. (2013). “Control of slippage with tunable bubble mattresses." Proceedings of the National Academy of Sciences, 110 (21): 8422-8426.

3. Visser, C.W., Tagawa, Y., Sun, C. \& Lohse, D. (2012). “Microdroplet impact at very high velocity." Soft Matter 8:10732-10737.

4. Tagawa Y., Oudalov, N., Visser, C.W., Peters, I.R., Van der Meer, D., Sun, C., Prosperetti, A. \& Lohse, D. (2012). "Highly focused supersonic microjets." Physical Review X 2 (3): 031002.

\section{Manuscripts in progress}

5. Pohl, R., Visser*, C.W., Römer, G.R.B.E., Sun, C., Huis in 't Veld, A.J. , \& Lohse, D. "Ejection regimes in picosecond laser-induced forward transfer of metals.” Under review.

6. Hendriks, J. Visser*, C.W., Henke, S., Leijten, J., Saris, D.B.F., Lohse, D., Sun, C., \& Karperien, M., "Optimizing cell viability in droplet-based cell deposition." Submitted.

7. Visser, C.W., Frommhold, P., Mettin, R., Wildeman, S., Sun, C., \& Lohse, D. "Dynamics of high-speed micro-droplet impact: numerical simulations and experiments at frame rates beyond $10^{7} \mathrm{fps}$.” Submitted.

\footnotetext{
${ }^{*}$ Contribution equal to first author.
} 
8. Visser, C.W., Pohl, R., Römer, G.R.B.E., Sun, C., Huis in 't Veld, A.J., \& Lohse, D. "Towards $3 D$ printing of pure metals by picosecond laser-induced forward transfer."

9. Gelderblom, H., Visser, C.W., Sun, C., \& Lohse, D. "Drop impact on solid substrates: bubble entrapment and spreading dynamics." Lecture notes, Soft Interfaces summer school, Les Houches, France. Submitted.

\section{Conference proceedings}

10. Pohl, R., Visser, C.W., Römer, G.R.B.E., Sun, C., Huis in 't Veld, A.J., \& Lohse, D. (2014). "Imaging of the ejection process of nanosecond laserinduced forward transfer of gold." Proceedings of LPM2014, Vilnius, Lithuania.

11. Pohl, R., Visser, C.W., Römer, G.R.B.E., Sun, C., Huis in 't Veld, A.J., \& Lohse, D. (2014). "High-resolution imaging of ejection dynamics in laserinduced forward transfer." SPIE2014, San Francisco, USA.

12. Pohl, R., Visser, C.W., Römer, G.R.B.E., Sun, C., Huis in 't Veld, A.J., \& Lohse, D. (2013). "Droplet ejection in laser-induced forward transfer: mechanism for droplet fragmentation.” LAMP2013, Jul 2013, Nigata, Japan.

13. Huis in 't Veld, A.J., Hoppenbrouwers, M.B., Giesbers, M., Pohl, R., Römer, G.R.B.E., Visser, C.W., Sun, C., \& Lohse, D. (2013). "Imaging of copper ejection in pico- and nanosecond Laser Induced Forward Transfer." 4M Conference, Oct 2013, San Sebastian, Spain.

\section{Reports}

14. Visser, C.W., Homan, L., Sun, C., Hoeksema, B., Lohse, D. (2013). "Evaluation report for project NEEDLEFREE” (ERC proof-of-concept grant).

15. Visser, C.W. (2013). "Jet injection technology overview”.

\section{Awards}

1. Klein, A.L., Visser, C.W., Bouwhuis, W., Lhuissier, H., Sun, C., Snoeijer, J. H., Villermaux, E., Lohse, D., and Gelderblom, H. (2014). "Laser impact on a drop", Gallery of Fluid Motion - Milton Van Dyke Award. 


\section{Grants}

1. "Cell deposition for clinically feasible tissue regeneration", (2014) Applicants: Karperien, M., Lohse, D., Saris, D.B.F., Visser, C.W. Funding agency: MIRA, Twente University.

\section{Seminars \& presentations}

\section{Invited seminars}

1. BAMM Labs, Harvard University Medical School, Boston, USA, Nov 2013.

2. Laboratoire Matiére et Systémes Complexes, Université Paris Diderot, France, May 2013.

3. ENSOR Meeting, Göttingen, Germany, Sep 12-13, 2013.

4. MSD, Oss, Dec 16, 2011.

\section{Conference presentations}

5. Biofabrication Workshop Pohang, Korea. Oct 2014.

6. TERMIS-AP, Deagu, Korea. Oct 2014.

7. FLOW14, Enschede, The Netherlands. May 2014.

8. Physics@FOM, Veldhoven, The Netherlands. Jan 2014. Live table-top droplet impact experiments.

9. American Physical Society, 66th Annual Meeting - DFD, Pittsburgh, USA, Nov 2013.

10. ASML Workshop, Enschede, The Netherlands. Jan 2013.

11.Physics@FOM, Veldhoven, The Netherlands. Jan 2013.

12. American Physical Society, 66th Annual Meeting - DFD, Pittsburgh, USA, Nov 2013.

13. American Physical Society, 65th Annual Meeting - DFD, San Diego, USA, Nov 2012.

14. American Physical Society, 64th Annual Meeting - DFD, Baltimore, USA, Nov 2011. 


\section{Poster presentations}

15. Physics@FOM Veldhoven, The Netherlands, Jan 2014.

16. JMBC Burgersdag Delft, The Netherlands, Jan 2012.

17. Physics@FOM Veldhoven, The Netherlands, Jan 2012.

\section{Group presentations}

18. Group seminar, Applications of microdroplet impact. Nov 2013.

19. Journal Club, Laser-matter interaction. Oct 2013.

20. Group seminar, Microdroplet impact: visualization, analysis and applications. Jun 2013.

21. Journal Club, Maximal deformation of an impacting drop. May 2012.

22. Group seminar, High-speed microdroplet impact on smooth solid surfaces. Nov 2011.

\section{Teaching}

Teaching assistant, University of Twente

1. MSc course Advanced Fluid Mechanics, Course coordinator: Jacco Snoeijer. Lecture on vorticity dynamics; supervision of class tutorials; grading of assignments. Sep - Nov 2012 \& 2013.

2. MSc course Experimental Techniques in Physics of Fluids, Course coordinator: Chao Sun.

Lecture on micro-Particle Image Velocimetry; supervision of 1-week lab assignments. Sep - Nov 2012.

Student supervision, University of Twente

Master thesis supervision (graduation date in brackets)

1. Thomas Bredenoord (expected: Jun 2015) "On the fluid mechanics of needlefree injection".

2. Ana Sofia Oliveira (Jul 2014) "Direct printing from microfluidic chips". Visiting Master student from Lissabon University, Portugal.

3. Jan Hendriks (Dec 2013) "Cell viability after spray deposition". 
4. Marise Gielen (Aug 2013) "Cell detachment by a submerged microjet".

5. Sander Haase (May 2012) "Transport over a bubble mattrass" (co-supervisor).

\section{Bachelor thesis supervision}

6. Boris Boom \& Hille Groendijk (2013) "Characterization of needle-free injection into soft matter and skin with continuous jets".

7. Martin Klein Schaarsberg (2012) "Laser-induced cavitation in light-absorbing liquids".

\section{Internship student supervision}

8. Pei-Hsun Tsai (Jul-Aug 2014) "Air entrainment by micro-droplet impact on a deep pool". Visiting PhD student from National Taiwan University, Taiwan.

9. Toshiaki Fukada (Aug-Oct 2014) "Shock wave visualization in droplet impact". Visiting PhD student from Osaka University, Japan.

\section{International collaborations}

1. Dr. Robert Mettin, Faculty of Physics, Georg-August-Universität Göttingen, Germany

2. Dr. Alexander Lippert, LAM Research AG, Villach, Austria

\section{Scientific community work \& memberships}

\section{Conference organization}

- FLOW14: 1st International Conference on Micro \& Nanofluidics: Organization of masterclasses by inviting key speakers, writing the program, and attracting students (2014).

\section{Seminar organization}

- Journal Club: Organizing the monthly presentation of a journal article by a group member (2011-2013). 


\section{Journal referee}

- Journal of Fluid Mechanics

- Experiments in Fluids

\section{Science popularization}

- Science Café: Table-top droplet impact experiments for a general audience.

\section{Attended summer schools \& courses}

- Soft Interfaces

Ecole de Physique, Les Houches, France. 2 to 27 Jul, 2012.

- Complex Motion in Fluids

Technical University of Denmark, Kogerup, Denmark. 7 to 13 Aug, 2011.

- J.M. Burgerscentrum (JMBC) courses:

- Particle Image Velocimetry, Technical University of Delft, The Netherlands, 24 to 28 Oct, 2011.

- Capillarity, University of Twente, The Netherlands, 9 to 13 May, 2011.

\section{Extra-cirricular activites}

- Studium Generale, University of Twente, The Netherlands (2012 - present). The Studium Generale organizes the dissemination of scientific results to a general audience. As a program committee member I contribute to setting the annual agenda of the Studium Generale. 
3D-printing, biofabrication, diesel engines, and spray cleaning all depend on controlled drop impact. However, surprisingly, these drops have received scarce attention so far. This is mainly due to their small size and high impact velocity, which makes visualizing the impact a challenge.

This thesis describes methods to generate and visualize the impact of single, high-speed (faster than $10 \mathrm{~m} / \mathrm{s}$ ) microdrops (smaller than $100 \mu \mathrm{m}$ in diameter). The impact dynamics are visualized in detail, and numerically modeled. The results are applied to optimize deposition of cell-containing drops used in biofabrication and burn treatment. Finally, by precisely controlling the impact of metal microdrops, fabrication of gold and copper micropillars is enabled. 